\title{
The Khovanov homology of knots
}

\author{
Giovanna Le Gros
}

VICTORIA UNIVERSITY OF WELLINGTON

Te Whare Wananga o te Upoko o te Ika a Maui

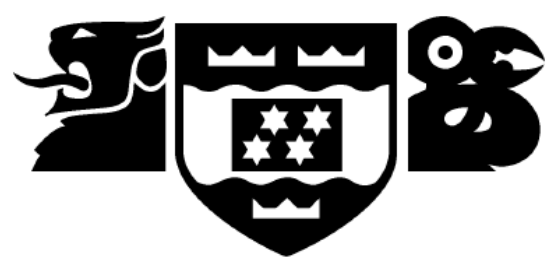

School of Mathematics and Statistics

Te Kura Mātai Tatauranga

\begin{abstract}
A thesis
submitted to the Victoria University of Wellington in fulfilment of the requirements for the degree of Master of Science in Mathematics.
\end{abstract}

Victoria University of Wellington 



\begin{abstract}
The Khovanov homology is a knot invariant which first appeared in Khovanov's original paper of 1999, titled "a categorification of the Jones polynomial." This thesis aims to give an exposition of the Khovanov homology, including a complete background to the techniques used. We start with basic knot theory, including a definition of the Jones polynomial via the Kauffman bracket. Next, we cover some definitions and constructions in homological algebra which we use in the description of our title. Next we define the Khovanov homology in an analogous way to the Kauffman bracket, using only the algebraic techniques of the previous chapter, followed closely by a proof that the Khovanov homology is a knot invariant. After this, we prove an isomorphism of categories between TQFTs and Frobenius objects, which finally, in the last chapter, we put in the context of the Khovanov homology. After this application, we discuss some topological techniques in the context of the Khovanov homology.
\end{abstract}





\section{Acknowledgements}

I would like to thank my supervisor, Geoff Whittle, for his never-ending eagerness to read my work, his encouragement and of course for sharing his vast mathematical and more general know-how. Also, I am very grateful for his willingness to supervise me. In addition, I must thank Rob Goldblatt for his help and advice in writing a chapter of this thesis.

For the choice of a thesis topic which I truly enjoyed exploring, I would like to thank Sharon Hollander, who also set me off on the right (mathematical) foot.

I could have not hoped for better office-mates, Jasmine and Susan, who were always energetic suppliers of food, distraction and general entertainment. Without them, I certainly would have never been able to create such a hazardous work-space.

More broadly, I would like to mention the others of the mathematics department, who have been here and there and everywhere in discussions and pursuits that were more or less mathematical. To name a few of the many, Del, Matt, sleepy Sam, Sam O., Tim and Will.

Furthermore, I am indebted to Michael W. for some unquestionably invaluable ATEX help. I would also like to more generally thank SMS for overall helpfulness, support, and providing such a friendly environment for a student.

To conclude, I want to thank my family, whose patience I perhaps tested. I could not have done without their support. 


\section{Contents}

\begin{tabular}{lll}
\hline & Introduction & 1
\end{tabular}

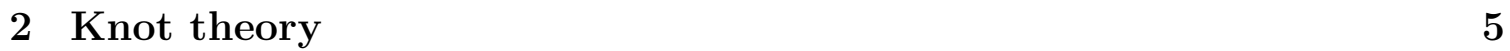

2.1 An introduction to knot theory . . . . . . . . . . . . . . 5

2.2 The Jones polynomial $\ldots \ldots \ldots$. . . . . . . . . . . . . . . . . . . . . . 17

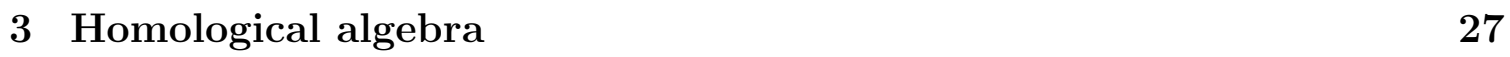

3.1 Background . . . . . . . . . . . . . . . . . . . . . . . . . . . . 28

3.2 Cochain complexes and cohomology . . . . . . . . . . . . . . . . . . 36

3.3 Z

\begin{tabular}{|lll}
\hline & The Khovanov homology & 63
\end{tabular}

4.1 Setting the scene . . . . . . . . . . . . . . . . . . . . . . . . 64

4.2 The Khovanov bracket . . . . . . . . . . . . . . . . . . . . . . . . . . 67

4.3 The Khovanov cohomology . . . . . . . . . . . . . . . . . . . . . . . 84

4.4 Relation to Jones polynomial . . . . . . . . . . . . . . . 85

4.5 A long exact sequence $\ldots \ldots \ldots$. . . . . . . . . . . . . . 87

$\begin{array}{lll}5 & \text { Invariance under the Reidemeister moves } & 93\end{array}$

$5.1 \quad$ First Reidemeister move $\ldots \ldots \ldots$. . . . . . . . . . . . . . . . . . . . . . 96 
5.2 Second Reidemeister move . . . . . . . . . . . . . . . . . . . . . . . . 101

5.3 Third Reidemeister move . . . . . . . . . . . . . . . . . . . . . . . . . 107

6 Topological Quantum Field Theories and Frobenius objects 113

6.1 Symmetric monoidal categories . . . . . . . . . . . . . . . . . . . 114

6.2 Frobenius objects $\ldots \ldots \ldots \ldots \ldots$. . . . . . . . . . . . 136

6.3 Cobordisms . . . . . . . . . . . . . . . . . . . . 150

$6.4 \quad(1+1)-\mathrm{TQFTs} \ldots \ldots \ldots \ldots \ldots \ldots \ldots$

6.5 The Equivalence of categories . . . . . . . . . . . . . . . . . . 165

6.6 Graded $R$-modules . . . . . . . . . . . . . . . . . . . . . . . . . . . 165

\begin{tabular}{lll}
\hline & TQFTs and the Khovanov homology & 169
\end{tabular}

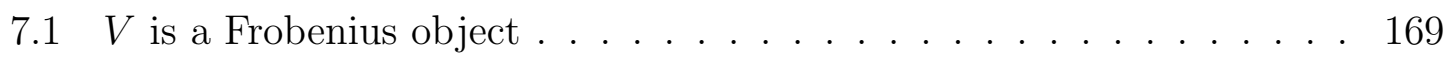

$7.2 \quad$ A topological $n$-dimensional cube of smoothings . . . . . . . . . . 172

7.3 The Khovanov bracket is a chain complex . . . . . . . . . . . . . . 173

7.4 Some properties of the TQFT $\mathcal{F}$ associated to $V$. . . . . . . . . 176

$7.5 \quad$ First Reidemeister move (again) . . . . . . . . . . . . . . . . . . . . . 179 


\section{Chapter 1}

\section{Introduction}

In 1999, Khovanov published his original paper titled "a categorification of the Jones polynomial", [6]. This categorification is what we today call the Khovanov homology. Before its introduction, there were no other knot invariants of this type, and specifically it is regarded as one of the first categorifications in knot theory. In this process of categorification, Khovanov found a homology theory that is a link invariant with excellent properties. For instance, it encodes the Jones polynomial and includes some of the perks of a homology theory, including functoriality, and more. It has prompted much research in knot homology theories as well as research into variants of Khovanov homology.

In the following years since Khovanov's original paper, Dror Bar-Natan wrote a paper on Khovanov homology in a more approachable form, along with computations of the Khovanov homology for some knots [1]. These computations were enough to show that the Khovanov homology is indeed a stronger knot invariant than the Jones polynomial. Another nice introduction to the Khovanov homology was written by Paul Turner [13].

Since then, some other neat properties of the Khovanov homology have emerged. In particular, Kronheimer and Mrowka proved that the Khovanov homology is an unknot-detector. That is, an unknot is the only link that has the Khovanov homology of the unknot. Also, Dror Bar-Natan produced another influential paper, [2], 
which reintroduces the theory in a more geometric way.

This thesis gives an introduction to the Khovanov homology of knots. Unquestionably, there are a lot of interesting features of the Khovanov homology, however, this thesis is restricted to its definition and its hallmark properties. While there is certainly a well written literature on this subject, our point of difference is that we assume less prerequisite knowledge, thus providing a more elementary groundwork. This includes a complete discussion of the homological algebra involved, and some foundations of knot theory. Additionally, we endeavour to describe the relationship between $(1+1)$-Topological Quantum Field Theories, or TQFTs, and Frobenius objects. The correspondence between these two collections (really an isomorphism of categories) was used in Khovanov's original paper, and is helpful to understanding his definition.

From the outset, our aim was to give a relatively complete exposition of the mathematical techniques we use in the definition of the Khovanov homology. Certainly one can completely describe the Khovanov homology algebraically, but the motivations for why the algebraic rules are what they are, are somewhat demystified by applying a TQFT. Also, routine justification of well defined-ness of the Khovanov bracket is vastly simplified by applying a TQFT. Hence, at some point during my research, it became clear that some of the geometric motivation of the definition was unclear without understanding the relationship between TQFTs and Frobenius objects. Therefore, we undertook the task of trying to understand in detail the categorical equivalence between TQFTs and Frobenius objects following [7].

It is true that the resulting thesis provides a slightly fuller picture than necessary for our purposes. This was an outcome of chapters taking on a life of their own, and is particularly true for the three background chapters, the chapter on knot theory, Chapter 2, the chapter on homological algebra, Chapter 3, and the chapter on TQFTs, Chapter 6.

Moving forward, we now outline how we will proceed. We start with some background knot theory to give some meaning to the "knot invariant" part of the title. 
Next we cover some fundamentals of homological algebra, which, unsurprisingly, will be essential in discussing the "homology" part of the title. This is followed by the title chapter, in which we discuss the Khovanov homology, followed closely by a chapter which contains the proofs that the Khovanov homology is, as promised, a knot invariant. At this point, the thesis in some sense comes to a natural stopping point, where the Khovanov homology is defined purely as an algebraic object, with some rather mysteriously constructed objects, in particular, a graded vector space $V$. We will not stop here. As it turns out, this object is part of a collection of objects which are in bijective correspondence with a more topological object (the aforementioned TQFT). Hence, the next chapter takes this route, and provides a proof of this correspondence. We then apply the results of this chapter to the Khovanov homology, where we tie off some loose ends.

Explicitly, the chapters are as follows. We begin by introducing some of the main concepts and objectives of knot theory in Chapter 2. In this chapter we also define some useful terminology when discussing knots. Section 2.2 is devoted to the Jones polynomial which we define and prove is a knot invariant. It is worth noting that the definition of the Kauffman bracket is a foreshadowing of a construction that appears in a later chapter.

In Chapter 3, we cover some fundamental concepts in algebra. In particular, an important operation we will use is the tensor product, $\otimes$. Also, to name a few here, we define "chain complexes", "homology", "chain homotopies", and "cone constructions". In the last section of this chapter, we define graded vector spaces and adapt some of our descriptions to this case. This will be important in the construction of the Khovanov homology.

After Chapter 3, we are in a position to define the Khovanov homology, which we do promptly in Chapter 4. We define it and then state some of its nice properties in the latter part of this chapter. Here, we also restate some of the nice features of the Khovanov homology. Unfortunately, this chapter does not give the definition in its entirety, as we will not prove that the Khovanov bracket is well defined until the last chapter, Chapter 7 . 
Next, we prove Reidemeister invariance of the Khovanov homology in Chapter 5 . The proofs follow Dror Bar-Natan's method ([1]) closely. We have ventured to do so very explicitly with additional explanations and leave no stone unturned. These moves only require algebraic techniques, which is nice. However, this is not the method of Khovanov, nor are the algebraic techniques particularly intuitively enlightening, but serves its purpose as a verification of the invariance of the Khovanov homology under the Reidemeister moves.

In Chapter 6, we begin discussion of TQFTs from the categorical point of view. We will assume some knowledge of category theory. We begin by describing symmetric monoidal categories and functor categories, and show an isomorphism between categories of this type. The first few sections are done generally, without considering specific categories. After proving the equivalence, we show that the category we used in the construction of the isomorphism has exactly the attributes of a category with topologies as objects and morphisms, the category of 2-cobordisms. Our conclusion is that there is an isomorphism of categories between Frobenius objects and TQFTs.

Lastly, in Chapter 7, we show that we can indeed apply a TQFT in Khovanov homology, and hence can replace $R$-homomorphisms with cobordisms. Hence, algebraic formulae are replaced by pictures of surfaces. We also return to the missing proof we left in Chapter 4, and prove that the Khovanov bracket is well defined. Lastly, we use TQFTs to prove again the invariance of the Khovanov homology under the first Reidemeister move. Finally, at this point, we hope to have encouraged some geometric intuition of the construction of the Khovanov homology.

An omission from this thesis is a computation of the Khovanov homology of a knot. I have computed the Khovanov homology of a class of torus knots, but unfortunately due to the length of this thesis have had to leave it out. My only justification for choosing not to include this chapter in particular is the feeling that excluding any other chapter would leave a gap in the story. 


\section{Chapter 2}

\section{Knot theory}

The Khovanov homology is a knot invariant, so we begin with an overview of knot theory. The beginning of this chapter, in which we discuss the basic concepts of knot theory, follows the arrangement of [3]. Next we introduce some standard theorems and techniques in knot theory such as Reidemeister's theorem. In the last section of this chapter, we introduce the Kauffman bracket, which is used to define the Jones polynomial. Our normalisation here is not standard, but is done in anticipation of the Khovanov homology.

\subsection{An introduction to knot theory}

We begin by defining the concepts we need to define what exactly we mean by a "knot".

Recall that a homeomorphism of topological spaces is a bi-continuous bijection between spaces. Let $X, Y$ be topological spaces. An embedding is a map $f: X \hookrightarrow Y$ such that $f$ is a homeomorphism from $X$ to its image, $f(X) \subseteq Y$. 


\section{Homotopy}

Recall $I$ is the closed interval $[0,1]$. For topological spaces $X$ and $Y$, consider continuous maps $f, g: X \rightarrow Y$. A homotopy $H: X \times I \rightarrow Y$ from $f$ to $g$ (also written $f \rightarrow g)$ is a continuous map such that:

1. $H(x, 0)=f(x)$ for all $x \in X$

2. $H(x, 1)=g(x)$ for all $x \in X$.

Note that the maps $h_{t}(x):=H(x, t)$ for every $t \in I$ are also continuous maps from $X$ to $Y$. If there exists a homotopy between maps $f, g: X \rightarrow Y$, then $f$ and $g$ are called homotopic. This is an equivalence relation on maps from $X$ to $Y$.

Suppose $H$ is a homotopy, and let $h_{t}:=\left.H\right|_{X \times\{t\}}$. If $h_{t}$ is also an embedding from $X \times\{t\}$ to $Y$ for each $t \in I$, then $H$ is called an isotopy.

\section{Ambient isotopy}

Let $f: X \hookrightarrow Y$ and $g: X \hookrightarrow Y$ be embeddings. An ambient isotopy from $f$ to $g$ is an isotopy $H: Y \times I \rightarrow Y$ from the identity on $Y$, $\operatorname{id}_{Y}$, to the map that brings $\operatorname{im}(f)$ to $\operatorname{im}(g)$. In other words, $H: Y \times I \rightarrow Y$ is an ambient isotopy if it satisfies the following.

1. $H(y, 0)=y$ for all $y \in Y$

2. $H(f(x), 1)=g(x)$ for all $x \in X$

3. $h_{t}:=\left.H\right|_{Y \times\{t\}}: Y \times\{t\} \rightarrow Y$ is a homeomorphism for every $t \in I$.

If there exists an ambient isotopy between two maps, then these maps are called ambient isotopic. Ambient isotopy is an equivalence relation on embeddings $X \hookrightarrow Y$. 

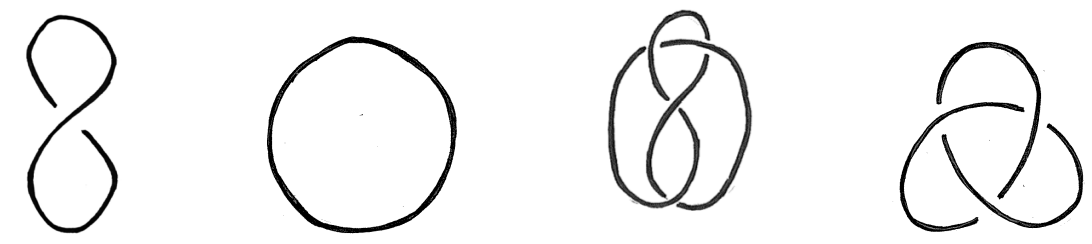

Figure 2.1: Some examples of knots. From left to right: two unknots, a figure eight knot, and a right-handed trefoil.

\section{Knots}

A knot is an embedding $K$ of the circle $S^{1}$ in $\mathbb{R}^{3}$. There is some abuse of terminology when referring to a knot. One can mean one, or even a few of many things: an embed$\operatorname{ding} K: S^{1} \hookrightarrow \mathbb{R}^{3}$, the equivalence class of embeddings $[K]_{\sim}$ under ambient isotopy, or even a knot diagram, all of which will be discussed further below. Nonetheless, for each of these meanings of "knot", two knots are considered the "same" knot if their corresponding embeddings are ambient isotopic. Many of the definitions in this section are from [3].

Intuitively, there is a natural equivalence on knots which captures the idea that we could move from one to the other without breaking the circle. For example, this equivalence should ensure that the two leftmost knots in Figure 2.1 are the same knot. Also, we want that the unknot, figure eight knot, and the trefoil knot are all in different equivalence classes, as one cannot manipulate one to look like any of the others. With all this in mind, we informally name the simplest knot (or rather the equivalence class of knots) the unknot, which is just a simple circle, which can be seen in Figure 2.1.

Let $X$ and $Y$ be topological spaces. Recall that the disjoint union of $X$ and $Y$, denoted $X \amalg Y$, is the disjoint union of their underlying sets with a natural topology which we define as follows. A set $U \subseteq X \amalg Y$ is open if and only if $U \cap X$ is open in $X$ and $U \cap Y$ is open in $Y$. A link is an embedding of the disjoint union of finitely many circles $\coprod_{k=1}^{n} S_{k}^{1}$ into $\mathbb{R}^{3}$. 

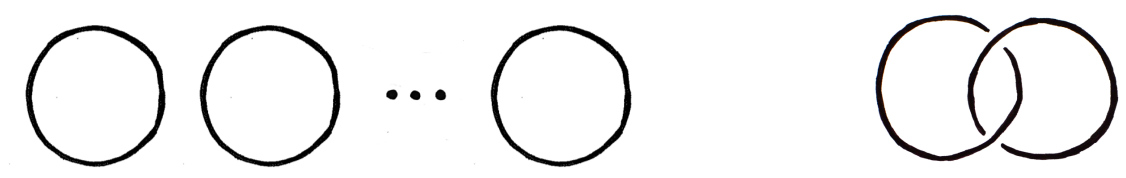

Figure 2.2: Here are some examples of links. On the left $k$ unlinked unknots. On the right is the Hopf link

The simplest link with $k$ components is $k$ unlinked unknots, which are links with "unknotted" and "unlinked" components. We define $k$ unlinked unknots to be the equivalence class of knots containing the knot in Figure 2.2. Disjoint union of knots has not been formally defined yet (see Definition 2.1.1), but we note that we can more formally define the link ' $k$ unlinked unknots' to be the disjoint union of $k$ unknots in $\mathbb{R}^{3}$.

A component of a link is the embedding of a single circle $S^{1} \subseteq \coprod_{k=1}^{n} S_{k}^{1}$ in the disjoint union, and an embedding of $\coprod_{k=1}^{n} S_{k}^{1}$ is said to have $n$ components. Unless specified, "knots" (by the previous definitions, links with exactly one component) are used to mean both links and knots. This is a common convention in knot theory which will be used here.

Note that general isotopy is not a strong enough condition to define an equivalence of knots, as we desire. To see why, we claim the diagram below give an example of a non-ambient isotopy between the unknot and the trefoil knot.

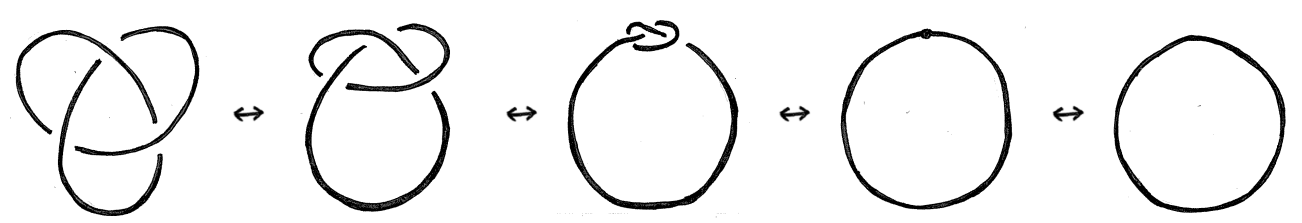

Every one of the above knots (and everything in between) is an embedding of a circle in $\mathbb{R}^{3}$. To see that these diagrams illustrate an isotopy from the trefoil to the unknot, 
the only fact that remains to show is that it is continuous at the "limit point" of the trefoil, call it $x \in \mathbb{R}^{3}$. Every 3-ball centred at $x, B(x, \epsilon)$, contains a trefoil, and therefore its pre-image in $S^{1} \times I$ will be open, as required.

Ambient isotopy ensures the space just around the knot, or a neighbourhood of the embedding is preserved. One can think of an ambient isotopy between knots as a deformation of one knot in $\mathbb{R}^{3}$ and the space around it into the other.

Not all embeddings of $S^{1}$ in $\mathbb{R}^{3}$ have "nice" properties. Wild knots are knots with infinite knotting - they have repeating sections of the knot that become smaller and smaller until they reach a limit point. To avoid dealing with these knots, we will consider only knots which are ambient isotopic to simple closed polygons in $\mathbb{R}^{3}$. These are called tame knots.

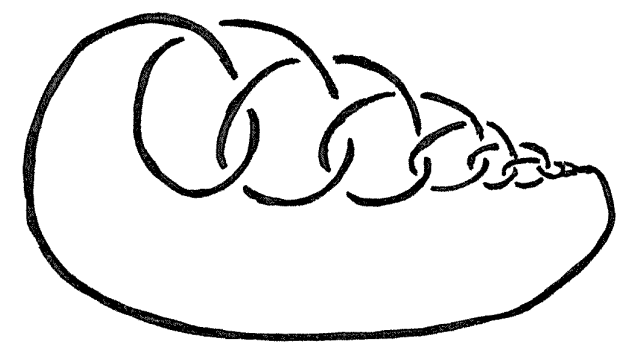

\section{Knot projections}

Since knots are three dimensional objects, they can be difficult to visualise. In order to simplify working with knots, we consider their projections onto a plane. More precisely, a knot projection of a knot $L$ is a projection of $L \subset \mathbb{R}^{3}$ onto the plane $\mathbb{R}^{2}$ which is injective except at finitely many points, $F \subset \mathbb{R}^{2}$. The pre-image of each point in $F$ has a cardinality of exactly two, with nice properties. For each of these points, we note which arc of $S^{1}$ is over and which is under on the plane.

The second-to-last statement, that the pre-image of each point in $F$ has a cardinality of two and has nice properties, ensures that there will be no places in the 
projection where the placements of the strands are not clear, such that three strands pass through a point, or that one strand does not only "skim" the other — each strand "passes through" the other. Note that since we are only considering tame knots, for every tame knot there exists a projection with finitely many crossings, since every tame knot is (by definition) ambient isotopic to a simple closed polygon.

There are many different knot projections for each knot, (for example, the figure below contains three unknots). A natural question to ask is what changes can one make to the knot projection, to ensure it is still a projection of the original knot. Before this discussion, we will describe some standard language and conventions when referring to knot diagrams.
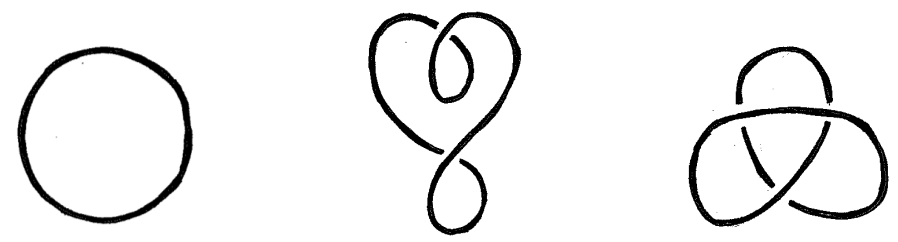

Knot projections will be referred to as diagrams, or sometimes as knots.

Consider the knot $K: S^{1} \hookrightarrow \mathbb{R}^{3}$. A section of a single knot, or more formally, for connected $A \subset S^{1}$ (an arc of the circle), the image of $A, K(A)$, is referred to as a strand. The points of $F$ are called the crossings of the knot diagram $D$. Hence, the statement about the cardinalities of the pre-images of elements in $F$ simply requires that each crossing has only two strands.

The crossings in the knot projection cannot be drawn continuously without losing important information about the knot. Therefore, the under-strands of a crossing are drawn 'broken', where the over-strand passes through.

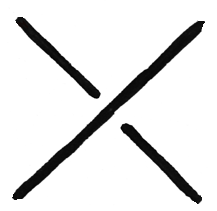


For the projection of a knot, the analogue for ambient isotopy is planar isotopy. This is an isotopy of the knot projection in $\mathbb{R}^{2}$ to another knot projection in $\mathbb{R}^{2}$. Note we must think of the actual projection - so each crossing will be a cross with no gaps. An isotopy between two knot projections requires that no crossings can pass through each other, as the image of the knot would cease to be an embedding

Clearly, planar isotopies do not describe all ambient isotopies of knots in $\mathbb{R}^{3}$, or, more importantly, if two projections represent equivalent knots. Therefore, we need to describe other manipulations of knot projections to include what knot projections are equivalent under ambient isotopy.

When naming knots, or referring to knots, we are referring to the entire equivalence class of knots. So, we can now formally define the unknot as the class of knots which are ambient isotopic to a knot diagram with no crossings. This can be sometimes ambiguous, as one can not always tell if two knots will resemble each other after ambient isotopy.

We now define the disjoint union of knots which is similar to that of topological spaces, but considers the embedding of the knots as well.

\section{Definition 2.1.1. Disjoint union of two knots}

Consider two knots or links $K_{1}$ and $K_{2}$ with knot diagrams $D_{1}$ and $D_{2}$ respectively. The knot $K$ is the disjoint union of $K_{1}$ and $K_{2}$ if there exists a knot projection $D$ of $K$ such that every crossing either belongs to $K_{1}$ or $K_{2}$. This knot is unique, and is written $K_{1} \amalg K_{2}$. In the case of knot diagrams, we use $D_{1} \amalg D_{2}$, or sometimes $D_{1} D_{2}$ to represent the knot diagram where every crossing either belongs to $D_{1}$ or $D_{2}$.

This definition can be extended to the disjoint union of a finite number of knots.

Next, we define the Reidemeister moves. This means we can forget about knots as embeddings and ambient isotopies and only work with projections as follows. 

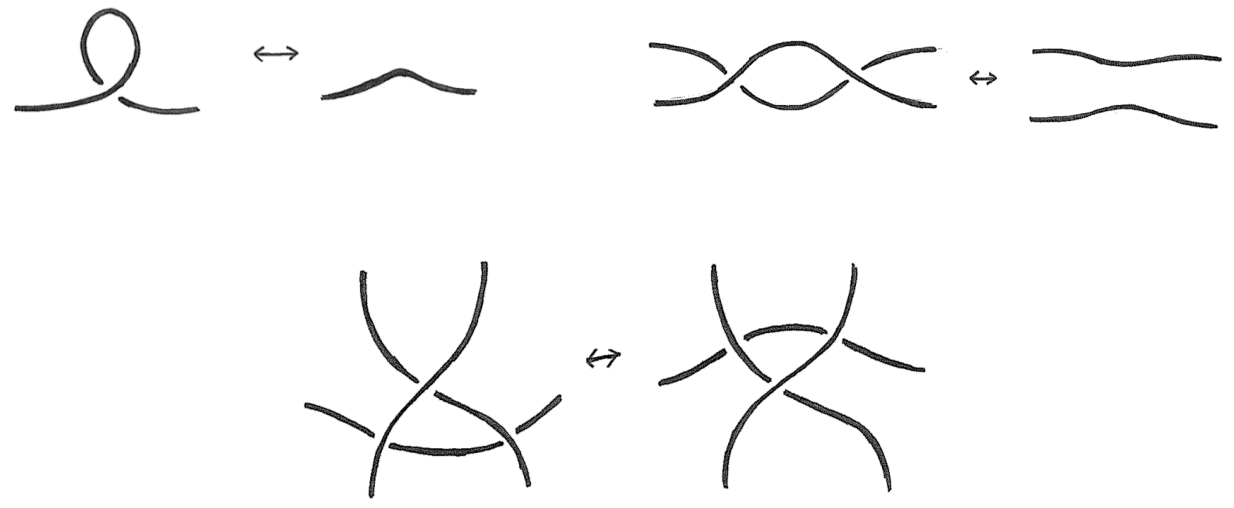

Figure 2.3: A minimal set of the three Reidemeister Moves: from one to three clockwise from top left

\section{Reidemeister moves}

The three Reidemeister moves are manipulations of knot diagrams. They are:

R1 Untwisting a left loop or a right loop,

R2 Moving one strand over or under another strand,

R3 Moving a strand over or under a crossing.

Strictly speaking, they are equivalences of knot diagrams, but we will refer to them as moves. The Reidemeister moves are used in, perhaps, one of the most important theorems in knot theory, as follows.

Theorem 2.1.2. Reidemeister's Theorem Two knots are equivalent under ambient isotopy if and only if they are equivalent under the Reidemeister moves and planar isotopy.

Reidemeister's Theorem gives us no way to tell if two knots are the equivalent from their knot projections. Rather, it tells us that if there exists a series of Reidemeister 
moves that changes one knot diagram to the other, then the two diagrams represent the same equivalence class of knots. Moreover, as there is no bound on the number of Reidemeister moves in the series which changes one to the other, this theorem is not useful to show that two knot projections are not equivalent.

These moves (there are in fact five equivalences) are not a minimal set of moves. Consider the set of the three Reidemeister moves in Figure 2.3 - which are limited to untwisting a right twist, moving one strand over a crossing, and moving a strand under a crossing. From only these three moves, the rest of the Reidemeister moves are also permitted.

A left-twist is equivalent to its untwisted form using a right-twist in the first move and R2.

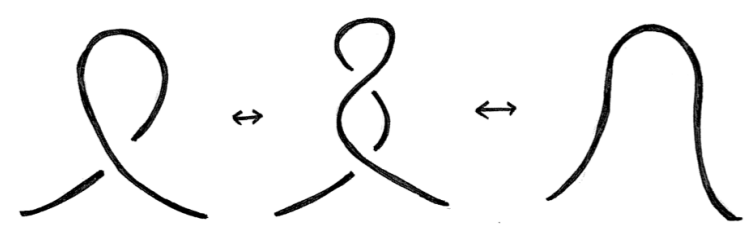

Moving a strand over a left crossing is equivalent moving a strand over a right crossing and two applications of $\mathbf{R} 2$.

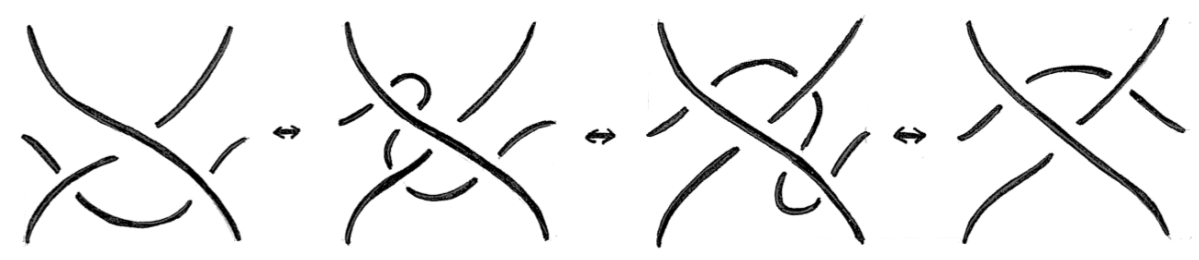

Finally, we claim passing a strand over follows from passing a strand under a crossing. This can be seen by planar isotopy, rotating the knot so the tangle in question looks like R3, it follows that moving a strand over a crossing is also permitted.

This minimal set of Reidemeister moves is not unique, but it will be useful when proving theorems about all the Reidemeister moves, which can be shown by only showing for the minimal set. Therefore, when proving theorems about invariance the 
Reidemeister moves, we observe that it is sufficient to only prove invariance for this minimal set, and will do so without further comment.

\section{Definition 2.1.3. Knot invariant}

A knot invariant is a function $\Psi:\{$ Links $\} \rightarrow \mathcal{S}$ such that if $K_{1}$ and $K_{2}$ are two equivalent knots, then $\Psi\left(K_{1}\right)=\Psi\left(K_{2}\right)$. Note that it is not required that $\Psi\left(K_{1}\right)=\Psi\left(K_{2}\right)$ implies that $K_{1}$ and $K_{2}$ are equivalent.

To prove a function $\Psi$ on the collection of links is a knot invariant, it is necessary and sufficient to prove that $\Psi$ is invariant under the three Reidemeister moves.

The knot invariants that we will be working are defined on oriented knot projections.

\section{Orientation of a knot}

An oriented knot is a knot which has an orientation. More formally, suppose a simple closed polygon $P$ has an ordered numbering of its vertices from 1 to $k$. Recall that a tame knot $K$ with one component is ambient isotopic to an embedding of a simple closed polygon $P$. Then the direction of increasing vertices of $P(\bmod k)$ defines an orientation of the knot, and is often denoted by an arrow in the direction of increasing vertices.

To obtain an orientation of a link, one assigns the orientation of each component separately.

It is clear from the diagrams in Definition 2.3 that the Reidemeister Moves are the same for oriented knots. Specifically, consider the collection of moves R1, R2, R3 with all possible orientations of strands. Hence, it follows that the "oriented" Reidemeister moves and planar isotopy are necessary and sufficient to say when two oriented knots are equivalent.

A knot is called invertible if there exists an ambient isotopy between an oriented knot and the same knot with the reverse orientation. All knots with less than eight 
crossings are invertible, which means only when looking at more complicated knots do differences appear between oriented and unoriented knots.

Note that it is much easier to find a link (with more than one component) that can be oriented in different ways to give distinct oriented knots. For example, consider the Hopf Link in Figure 2.2 with an arbitrary orientation of each component. Reversing the orientation of only one component of the link will create a new oriented link distinct from the first.

\section{Tangles and skein relations}

Sometimes it is useful to restrict one's attention to only one part of the knot projection, or to look how altering one part of the knot projection changes the knot.
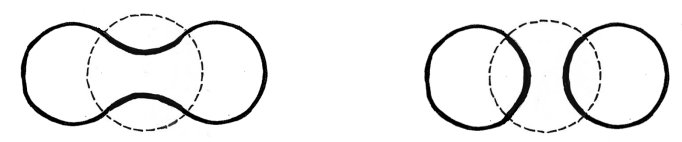

More formally, consider an embedding $\mathcal{B}$ of the open unit 3-ball, $B(x ; 1):=\left\{y \in \mathbb{R}^{3}\right.$ : $|x-y|<1\}$, in $\mathbb{R}^{3}$, and knot $K: S^{1} \hookrightarrow \mathbb{R}^{3}$. A 2-tangle $\mathcal{B}:=\mathcal{B}(B(x ; 1))$ is the image of the embedding $\mathcal{B}$ when the intersection $\mathcal{B}(B(x ; 1)) \cap K\left(S^{1}\right) \subseteq \mathbb{R}^{3}$ is homeomorphic to two disjoint open arcs.

There exists a projection of a knot containing a 2-tangle $\mathcal{B}$ onto the plane that contains only the strands of $\mathcal{B}$. This is equivalent to considering an embedding of an open unit 2-ball into the plane $\mathbb{R}^{2}$ where the strands of the knot cross the boundary of the 2-ball at exactly four distinct points. One can arrange these intersections on the perimeter to be evenly spaced, as the knots are invariant under planar isotopy of their respective knot diagrams.

An elementary 2-tangle is a 2-tangle with no crossings or exactly one crossing, of which there are exactly four, as seen in the figures below. In general, a 2-tangle can 
have any number of crossings.

Consider a knot with an identified elementary 2-tangle within the knot. Changing this tangle with any of the three other elementary 2-tangles will change the knot, and, informally, the relationship between a knot and knots with different elementary 2 -tangles are known as skein relations. These skein relations are very useful when defining relationships between these knots.

The following are the four possible way to arrange an elementary 2-tangle in a knot. Clearly, they form four different knots.
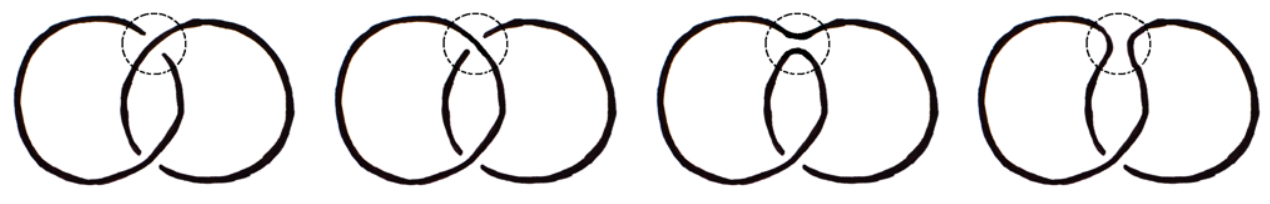

When talking broadly about knots that differ by only a elementary 2-tangle in exactly one place, it is convenient only to draw the elementary 2-tangle. We will refer to them as $a, b, c, d$ for the next few paragraphs.

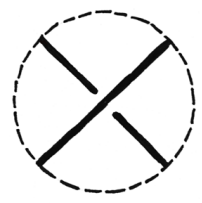

$a$

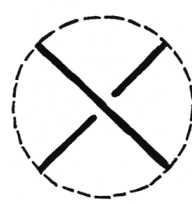

$b$

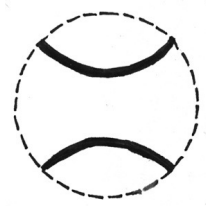

$c$

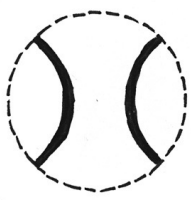

$d$

For example, consider a knot invariant $\Psi$, and without loss of generality suppose a knot diagram $D$ has an elementary 2-tangle $a$. Consider the knot $D$ with the elementary 2-tangle $a$ replaced with the elementary 2-tangle $b$. This knot is denoted $D_{b}$, and similarly for $D_{c}, D_{d}$. More formally than previously, a skein relation is when one can find, for example, $\Psi(D)$ in terms of $\Psi\left(D_{b}\right), \Psi\left(D_{c}\right)$, and $\Psi\left(D_{d}\right)$ (we say broadly 'a relation', since we don't know anything about the knot invariant $\Psi$ ). When we generalise a relation between knots with replaced tangles, we refer to the knots as 
simply what occurs at the tangle in question, as follows.

Note that it important to notice the surroundings of the crossing, as rotating one of the tangles by $\frac{\pi}{2}$ will change the knot.

This notation is consistent with oriented diagrams. To differentiate between the two types of oriented crossings, in the diagrams below the left crossing is a positive crossing and the crossing on the right crossing is a negative crossing. This is also known as the sign of the crossing.

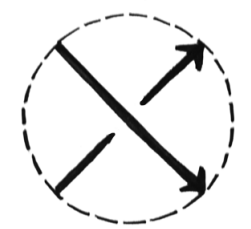

A positive crossing, $n_{+}$

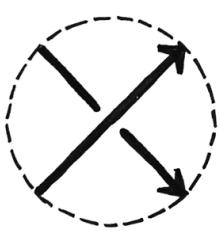

A negative crossing, $n_{-}$

We more generally use "tangles" to refer to a piece of a knot diagram.

\subsection{The Jones polynomial}

First we will define the Kauffman bracket. This method gives a nice analogue to how we will define the Khovanov homology.

\section{Definition 2.2.1. The Kauffman bracket}

Recall that a Laurent polynomial over the ring $R$ is a finite sum $\sum_{k=m}^{n} a_{k} x^{k}$ where $a_{k} \in R, m, n \in \mathbb{Z}$ (in other words, we allow negative powers of $x$ ). The Kauffman bracket of a knot diagram $D$, written $\langle D\rangle$, is a Laurent polynomial in the variable $q$ with coefficients in $\mathbb{Z}$. It satisfies the following three relations.

$\mathbf{K f} \mathbf{1}\langle\emptyset\rangle=1$

$\mathbf{K f 2}\langle\bigcirc \amalg D\rangle=\left(q+q^{-1}\right)\langle D\rangle$ 
$\operatorname{Kf} 3\left\langle\backslash^{\prime}\right\rangle=\langle\asymp\rangle-q\langle)(\rangle$

Here, $\emptyset$ denotes the empty link.

The first relation, Kf $\mathbf{1}$, defines the Kauffman bracket of the empty link. The second relation, Kf2, defines what the Kauffman bracket of the disjoint union of an unknot and $D$, in terms of the Kauffman bracket of $D,\langle D\rangle$. The third relation, Kf $\mathbf{3}$, gives us the Kauffman bracket of a knot $D$ in terms of the Kauffman brackets of the knots with one less crossing.

Note that Kf3 is equivalent to $\langle\aleph\rangle=\langle\rangle\rangle-q\langle\asymp\rangle$, which one can see by simply rotating every tangle by $\frac{\pi}{2}$. By applying $\mathbf{K f} \mathbf{3}$ recursively, one can reduce all knots to a sum of Kauffman brackets of unlinked unknots.

The relations $\mathbf{K f} \mathbf{1}$ and $\mathbf{K f} \mathbf{2}$ specify the Kauffman bracket of $k$ unlinked unknots.

$$
\langle k \text { unlinked unknots }\rangle=\left(q+q^{-1}\right)^{k}
$$

The Kauffman bracket is not itself a knot invariant, but a normalisation of it after orienting the knot (the Jones polynomial!) is. This will be defined soon.

\section{Total smoothings of a knot}

A smoothing of a knot diagram $D$ is when a crossing $\backslash$ in $D$ is replaced with either $\mathrm{a} \asymp$ or ) (. The smoothing $\asymp$ is called as the 0 -smoothing, and the smoothing $)($ is called 1-smoothing.

A total smoothing occurs when every crossing in a knot is replaced with one of these smoothings. Note that a total smoothing will only consist of unlinked unknots, which we know the Kauffman bracket of. Also, since there are two ways to smooth each crossing, there are exactly $2^{n}$ total smoothings of each knot diagram.

In order to keep track of the total smoothings, we will describe a way to label them 
by elements $\alpha$ of $\{0,1\}^{n}$ (strings of length $n$ from the set $\{0,1\}$ ). First, we number the $n$ crossings in $D$ by $1, \ldots, n$ in some arbitrary way. In a total smoothing, the $i$ th crossing will be replaced either by the 0 -smoothing or the 1 -smoothing, and this corresponds, as you would expect, to the 0 or 1 in the $i$ th place of $\alpha$. Hence, once we order the crossings of the knot diagram, there is a natural bijective correspondence between the total smoothings of a knot diagram with $n$ crossings and elements of $\{0,1\}^{n}$. We refer to the total smoothing associated to $\alpha$ as $S_{\alpha}$.

The number of occurrences of a 1 in the string $\alpha$ is called the height of the smoothing, denoted by $|\alpha|$ or $r_{\alpha}$. Also, the total 0-smoothing (respectively total 1-smoothing) refers to the smoothing $S_{00 \cdots 0}$ (respectively $S_{11 \cdots 1}$ ), the smoothing where every crossing is replaced by a 0 -smoothing (respectively 1 -smoothing).

We denote the number of cycles, or unknots in the smoothing $S_{\alpha}$ by $k_{\alpha}$, and therefore $\left\langle S_{\alpha}\right\rangle=\left(q+q^{-1}\right)^{k_{\alpha}}$.

\section{Example 2.2.2. 0011 smoothing of the Figure Eight Knot}
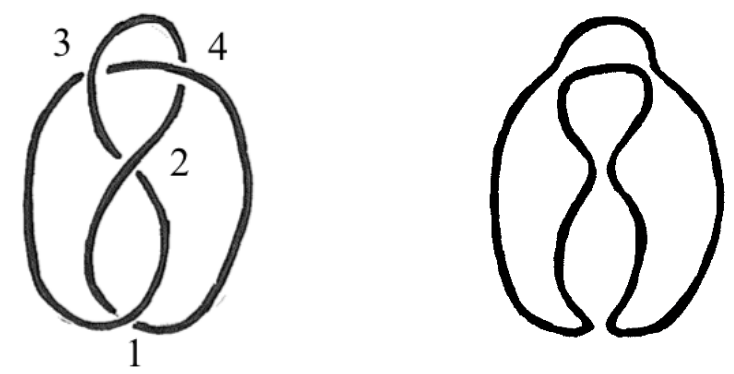

Arbitrarily numbered figure eight knot and its 0011 smoothing.

\section{The $n$-dimensional cube}

A construction that appears regularly during this thesis is the $n$-dimensional cube. Let $\alpha, \alpha^{\prime} \in\{0,1\}^{n}$ such that the strings $\alpha$ and $\alpha^{\prime}$ differ at exactly one place and $|\alpha|<\left|\alpha^{\prime}\right|$. We define the $n$-dimensional cube to be a directed graph with vertices labelled by elements of $\{0,1\}^{n}$, and directed edges $\left\{\alpha \rightarrow \alpha^{\prime}\right\}$ where $\alpha, \alpha^{\prime}$ are as defined in the previous sentence. For now, we only will be concerned with the vertices, but 


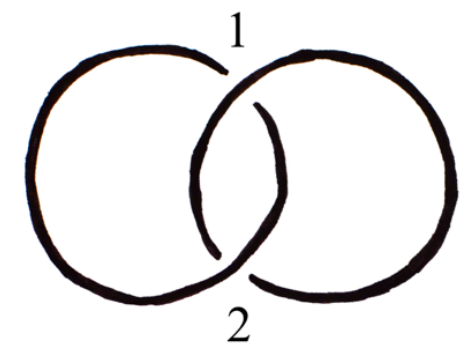

Figure 2.4: Hopf Link

the edges will reappear in later chapters.

Let $D$ be a knot diagram $D$ with $n$ crossings. In terms of the previous discussion, every total smoothing of $D$ can be associated with a vertex of the $n$-dimensional cube, and is drawn in Example 2.2.3.

Example 2.2.3. Computing the Kauffman bracket Here is an example how of the Kauffman bracket of a simple knot is computed using the 2-dimensional cube.

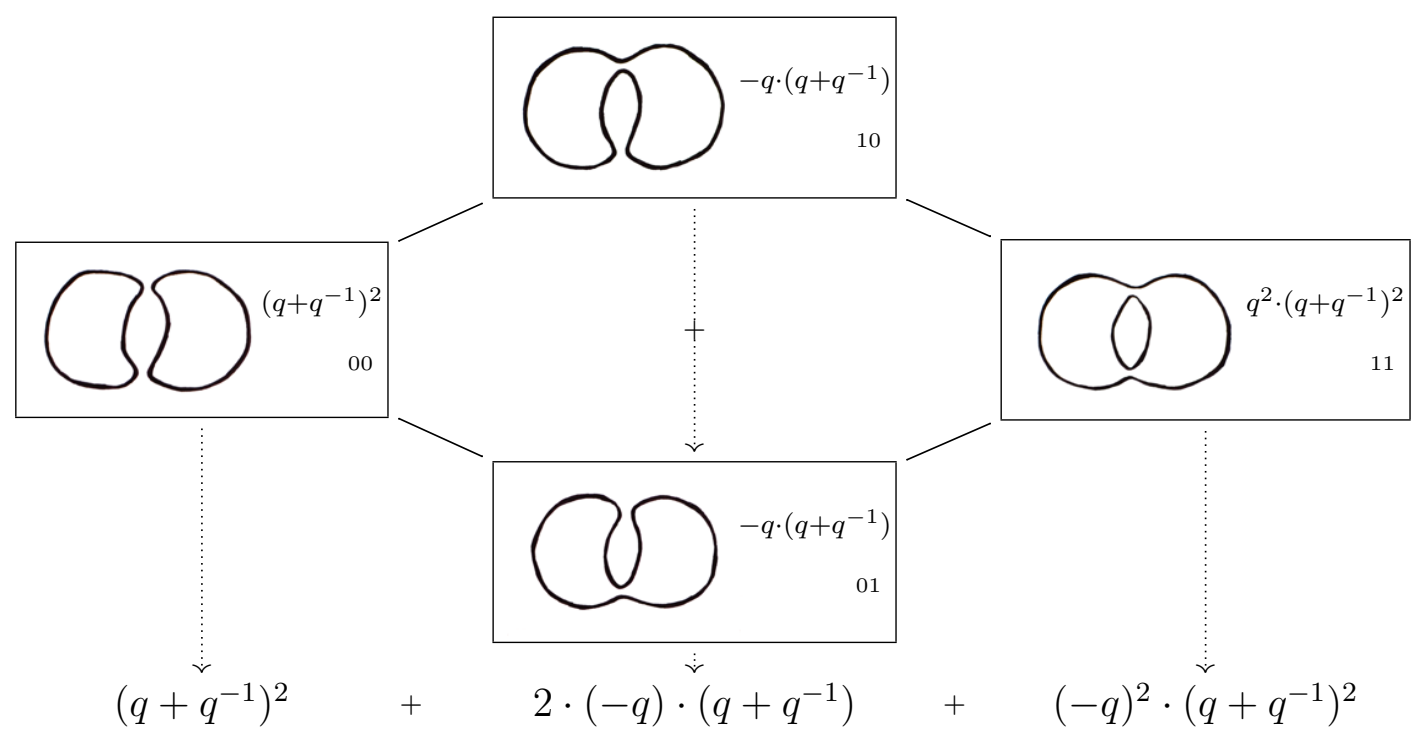


Here is the 2-dimensional cube with smoothings of the same height $|\alpha|$ on each vertical, with the total 0-smoothing on the leftmost vertical and the total 1-smoothing on the rightmost vertical. The polynomial in the box of each smoothing $S_{\alpha}$ is $(-q)^{|\alpha|}\left\langle S_{\alpha}\right\rangle$.

Using Kf 3, we see that the sum of these polynomials is the Kauffman bracket, since for every 1 -smoothing in the smoothing, we multiply by $(-q)$.

We now prove that the relations in 2.2.1 define the unique polynomial in Equation 2.1. This polynomial is defined in terms of the total smoothings of $D$.

Theorem 2.2.4. Let $D$ be a knot diagram with $n$ crossings. The relations in Definition 2.2.1 define a unique polynomial independent of the choice of the crossing when applying the third skein relation, $\boldsymbol{K} \boldsymbol{f 3}$, on D. Therefore, it is independent of how the crossings are numbered. These relations define the Kauffman Bracket of a knot diagram $D$ which can be written as the following sum.

$$
\langle D\rangle=\sum_{\alpha \in\{0,1\}^{n}}(-q)^{r_{\alpha}}\left(q+q^{-1}\right)^{k_{\alpha}}
$$

Proof. This will be shown by induction on the number of crossings of the knot diagram $D$.

As previously mentioned, if $D$ is a knot with no crossings and $k$ components, then the Kauffman bracket of $D$ is $\left(q+q^{-1}\right)^{k}$, which satisfies Equation 2.1.

For the induction hypothesis, we assume $\langle D\rangle=\sum_{\alpha \in\{0,1\}^{n}}(-q)^{r_{\alpha}}\left(q+q^{-1}\right)^{k_{\alpha}}$ is true for $n=k$ crossings. We now show it follows that it is true for $n=k+1$ crossings.

Consider a knot $D$ with $n$ crossings with an arbitrary numbering of the crossings. Choose the $i$ th crossing of $D$. Let $D_{0}$ be identical to $D$ but with a 0 -smoothing at the $i$ th crossing, and similarly $D_{1}$ be identical to $D$ but with a 1 -smoothing at the $i$ th crossing. The third relation of 2.2.1 states $\langle D\rangle=\left\langle D_{0}\right\rangle-q\left\langle D_{1}\right\rangle$.

By the induction hypothesis, one can assume that $\left\langle D_{0}\right\rangle=\sum_{\alpha \in\{0,1\}^{k}}(-q)^{r_{\alpha}}\left(q+q^{-1}\right)^{k_{\alpha}}$ 
and $\left\langle D_{1}\right\rangle=\sum_{\alpha \in\{0,1\}^{k}}(-q)^{r_{\alpha}}\left(q+q^{-1}\right)^{k_{\alpha}}$, where $k_{\alpha}$ depends on the knot in question (here, $D_{0}$ and $D_{1}$ respectively). First, we need to introduce some notation.

Let $\alpha_{j}$ for $1 \leq j \leq n$ be the $j$ th element of the string $\alpha \in\{0,1\}^{n}$. For some $\alpha \in\{0,1\}^{k}$, let $\alpha^{0}$ and $\alpha^{1}$ represent elements of $\{0,1\}^{k+1}$ such that the $i$ th element in the string $\alpha^{0}$ is 0 , and $\left(\alpha^{0}\right)_{j<i}=\alpha_{j}$ and $\left(\alpha^{0}\right)_{j>i}=\alpha_{j-1}$. Similarly, the $i$ th element in the string $\alpha^{1}$ is 1 , and satisfies $\left(\alpha^{1}\right)_{j<i}=\alpha_{j}$ and $\left(\alpha^{1}\right)_{j>i}=\alpha_{j-1}$. So $\alpha^{0}$ (respectively $\left.\alpha^{1}\right)$ represents a total smoothing of $D$ which is exactly the total smoothing $\alpha$ of $D_{0}$ (respectively $D_{1}$ ).

Then, let $k_{\alpha}^{0}$ be the number of cycles in $D_{0}$ with smoothing $\alpha$, and let $k_{\alpha}^{1}$ be the number of cycles in $D_{1}$. Then, $k_{\alpha}^{0}=k_{\alpha^{0}}$ and $k_{\alpha}^{1}=k_{\alpha^{1}}$. Note that $r_{\alpha}$ depends only on $\alpha$, and not on the smoothing itself, and that $r_{\alpha^{0}}=r_{\alpha}=r_{\alpha^{1}}-1$.

$$
\begin{aligned}
\langle D\rangle & =\left\langle D_{0}\right\rangle-q\left\langle D_{1}\right\rangle \\
& =\sum_{\alpha \in\{0,1\}^{k}}(-q)^{r_{\alpha}}\left(q+q^{-1}\right)^{k_{\alpha}^{0}}+(-q) \sum_{\alpha \in\{0,1\}^{k}}(-q)^{r_{\alpha}}\left(q+q^{-1}\right)^{k_{\alpha}^{1}} \\
& =\sum_{\alpha^{0} \in\{0,1\}^{k+1}}(-q)^{r_{\alpha} 0}\left(q+q^{-1}\right)^{k_{\alpha^{0}}}+(-q) \sum_{\alpha^{1} \in\{0,1\}^{k+1}}(-q)^{r^{1}-1}\left(q+q^{-1}\right)^{k_{\alpha} 1} \\
& =\sum_{\alpha^{0} \in\{0,1\}^{k+1}}(-q)^{r_{\alpha^{0}}}\left(q+q^{-1}\right)^{k_{\alpha^{0}}}+\sum_{\alpha^{1} \in\{0,1\}^{k+1}}(-q)^{r^{1}}\left(q+q^{-1}\right)^{k_{\alpha}} \\
& =\sum_{\alpha \in\{0,1\}^{k+1}}(-q)^{r_{\alpha}}\left(q+q^{-1}\right)^{k_{\alpha}}
\end{aligned}
$$

The last equality holds because $\left\{\alpha^{0}: \alpha \in\{0,1\}^{k}\right\} \cup\left\{\alpha^{1}: \alpha \in\{0,1\}^{k}\right\}=\{0,1\}^{k+1}$ and $\left\{\alpha^{0}: \alpha \in\{0,1\}^{k}\right\} \cap\left\{\alpha^{1}: \alpha \in\{0,1\}^{k}\right\}=\emptyset$.

We now claim the Kauffman bracket does not depend on the ordering of the crossings. Consider a permutation $\sigma:\{0,1\}^{n} \rightarrow\{0,1\}^{n}$ which changes the order of the crossings. The permutation will not alter the height of $\alpha \in\{0,1\}^{n}$, since there are still the same number of 1 s. Let $\alpha^{\prime}=\sigma(\alpha)$. Then, each smoothing $S_{\sigma(\alpha)}$ is the same 
as the smoothing $S_{\alpha^{\prime}}$. Hence, a smoothing of height $r$ will be the same as exactly one smoothing of height $r$ in the new ordering of the crossings. Therefore, summing over all total smoothings of height $r$ for each ordering will yield the same polynomial. Alternatively, we have the following equalities.

$$
\sum_{|\alpha|=r}\left\langle S_{\alpha}\right\rangle=\sum_{|\sigma(\alpha)|=r}\left\langle S_{\alpha}\right\rangle=\sum_{|\sigma(\alpha)|=r}\left\langle S_{\sigma(\alpha)}\right\rangle
$$

\section{Definition 2.2.5. The Jones polynomial}

Let $D$ be an oriented diagram with $n_{+}$positive crossings and $n_{-}$negative crossings. The unnormalised Jones polynomial of $D$, denoted $\hat{J}(D)$, is the following polynomial.

$$
\hat{J}(D):=(-1)^{n_{-}} q^{n_{+}-2 n_{-}}\langle D\rangle
$$

Theorem 2.2.6. The Jones polynomial is an invariant of oriented knots.

Proof. One only needs to show that the Jones polynomial is invariant under the three Reidemeister moves, as it is clearly invariant under planar isotopy.

First, we compute the Kauffman bracket of the tangle, and then assign all possible orientations to the strands to find the Jones polynomial.

I will use the fact that changing the orientation of a single strand in a crossing will change its sign as well. For example, by reversing orientations on both strands of a positive crossing, the crossing will remain a positive crossing.

1.

$$
\begin{aligned}
\langle\Omega\rangle & =\langle\Omega\rangle-q\langle\underline{O}\rangle \\
& =\langle\Omega\rangle-q\left(q+q^{-1}\right)\langle\Omega\rangle \\
& =\left(1-q^{2}-1\right)\langle\Omega\rangle \\
& =-q^{2}\langle\Omega\rangle
\end{aligned}
$$


We now assign an orientation to find the positive crossings and negative crossings. Suppose $\mathcal{R}_{x}$ has $n_{+}$positive crossings and $n_{-}$negative crossings. Then if $\Omega$ keeps the orientation of $\mathcal{X}$, then it has one less negative crossing. Therefore:

$$
\begin{aligned}
\hat{J}(\boldsymbol{\mathcal { X }}) & =(-1)^{n_{-}} q^{n_{+}-2 n_{-}}\langle\text {炎 }\rangle \\
& =(-1)^{n_{-}} q^{n_{+}-2 n_{-}}\left(-q^{2}\right)\langle\Omega\rangle \\
& =(-1)^{n_{-}-1} q^{n_{+}-2 n_{-}+2}\langle\Omega\rangle \\
& =(-1)^{n_{-}-1} q^{n_{+}-2\left(n_{-}-1\right)}\langle\Omega\rangle \\
& =\hat{J}(\boldsymbol{\Omega})
\end{aligned}
$$

as required.

The opposite orientation is almost identical, as assigning the reverse orientation to 8 , the sign of the crossing will be unchanged.

2 .

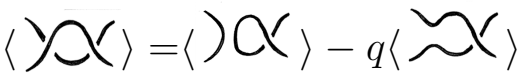

$$
\begin{aligned}
& =\langle) \widetilde{\sim}\rangle-q\langle) O(\rangle-q\langle\widetilde{\sim}\rangle+q^{2}\langle\precsim(\rangle
\end{aligned}
$$

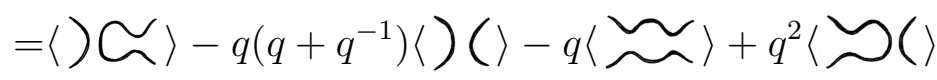

$$
\begin{aligned}
& =\left(1-q^{2}-1+q^{2}\right)\langle)(\rangle-q\langle\widetilde{\sim}\rangle \\
& =-q\langle\sim\rangle
\end{aligned}
$$

There are four ways to orient the strands in the tangle, and every possible orientation will have one positive crossing and one negative crossing.

Suppose 1 has $n_{+}$positive crossings and $n_{-}$negative crossings. Then will have $n_{+}-1$ positive crossings and $n_{-}-1$ negative crossings. Therefore: 


$$
\begin{aligned}
& \hat{J}(\text { Y) })=(-1)^{n_{-}} q^{n_{+}-2 n_{-}}\langle\text {Yे } \\
& =(-1)^{n_{-}} q^{n_{+}-2 n_{-}}(-q)\langle\stackrel{\sim}{\sim}\rangle \\
& =(-1)^{n_{-}-1} q^{n_{+}-2 n_{-}+1}(-q) \leadsto \sim \\
& =(-1)^{n_{-}-1} q^{n_{+}-2 n_{-}-1+2}(-q)\langle\stackrel{\sim}{\sim}\rangle \\
& =(-1)^{n_{-}-1} q^{\left(n_{+}-1\right)-2\left(n_{-}-1\right)}(-q)\langle\stackrel{\sim}{\sim}\rangle \\
& =\hat{J}(\stackrel{\sim}{\sim})
\end{aligned}
$$

3. We use planar isotopy and invariance under R2. That is, we use that $\langle\mathcal{C}\langle\rangle=$

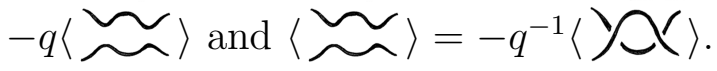

$$
\begin{aligned}
& \langle\rangle\langle\rangle=\langle\stackrel{\wedge}{\curvearrowright}-q\langle\rangle\langle\rangle \\
& =(-q)\langle\stackrel{\smile}{\bumpeq}-q\langle\rangle\langle\gamma\rangle \\
& =(-q)\left(-q^{-1}\right)\langle{\underset{\boldsymbol{q}}{\boldsymbol{\gamma}}}\rangle-q\langle\rangle_{\boldsymbol{\gamma}}\langle\rangle \\
& =\left\langle\mathscr{\vartheta}_{\boldsymbol{\gamma}}\right\rangle-q\langle\rangle\langle\rangle \\
& =\langle\rangle\langle
\end{aligned}
$$

Note that no matter how we orient number of positive crossings is the same as in $\hat{J}(\lambda)=\hat{J}(\lambda)$ for every possible orientation, as required.

Therefore, the Jones polynomial is invariant under the Reidemeister moves. 


\section{Chapter 3}

\section{Homological algebra}

In order to discuss the Khovanov homology and the proof that it is a knot invariant, we first need the tools of homological algebra. Much of this chapter can be generalised to a class of categories called abelian categories. In this chapter, for clarity and brevity of exposition, we will only refer only to $R$-modules but later in the chapter use the fact that it is possible to generalise to any abelian category. We will assume knowledge of the definition of a category and functors between categories, which can all be found in [10]. What we eventually need is everything in this section in terms of $\mathbb{Z}$-graded $R$-modules, which the last section of this chapter is devoted to.

The following definitions and propositions will only discuss cohomology rather than homology, which is all that is needed for the next chapters. Everything done here can be extended to homology with little effort. The more typical algebraic structures such as modules and tensor products follow [9] and [11. The homological algebra section follows the structure of the first chapter of Weibel, [14].

Notation 3.0.7. The arrow $\hookrightarrow$ denotes an injective map, or more generally, a monic morphism. The arrow $\rightarrow$ denotes a surjective map, or more generally, an epic morphism. 


\subsection{Background}

\section{Modules}

Let $R$ be a ring with a multiplicative identity. A left $R$-module $M$ is an abelian group $(M,+)$ and a scalar mulitplication map, $: R \times M \rightarrow M$, that satisfies the following relations for $r, s \in R$ and $m, n \in M$.

1. $r \cdot(m+n)=r \cdot m+r \cdot n$

2. $(r+s) \cdot m=r \cdot m+s \cdot m$

3. $(r s) \cdot m=r \cdot(s \cdot m)$

4. $1_{R} \cdot m=m$

A right $R$-module is defined similarly, but with a scalar multiplication map $M \times R \rightarrow$ $M$. We will only work with commutative rings with unit.

$R$-modules are generalisations of vector spaces, by replacing fields with rings. So, one can view a vector space as a $K$-module where $K$ is a field. Also, an abelian group $A$ can be considered a $\mathbb{Z}$-module where for $n \in \mathbb{Z}$ and $a \in A$, with scalar multiplication defined as $n \cdot a:=\underbrace{a+a+\cdots+a}_{n}$.

Consider left $R$-modules $M$ and $N$. A left $R$-homomorphism is a map $f: M \rightarrow N$ such that $f\left(m+m^{\prime}\right)=f(m)+f\left(m^{\prime}\right)$ for all $m, m^{\prime} \in M$ (that is, $f$ is a homomorphism of the groups $M$ and $N)$ and $f(r \cdot m)=r \cdot f(m)$ for all $r \in R$ and $m \in M$ (that is, $f$ is $R$-linear). An $R$-isomorphism is a bijective $R$-homomorphism, and two modules are called isomorphic, denoted $M \cong N$, if there exists an $R$-isomorphism between them.

A right $R$-homomorphism of right $R$-modules is defined similarly, but the $R$-linear condition is replaced with right $R$-multiplication, specifically $f(m \cdot r)=f(m) \cdot r$. When the context is clear, we use $R$-homomorphism, or simply homomorphism when 
referring to left or right $R$-homomorphisms. From now on, when we refer to $R$ modules, we mean left $R$-modules. This can all be translated into the language of right $R$-modules.

Let $M$ be a module. A submodule $N$ of $M$ is subset $N$ of $M$, which is closed under the binary operation + and action of $R$. In other words, $N$ is itself an $R$-module under the operations of the module $M$. Hence, $(N,+)$ is an abelian group, and $\left.\cdot\right|_{R \times N}: R \times N \rightarrow N$ is well defined, so $N$ is an $R$-module. The trivial $R$-module is the zero module, written 0 .

The collection of $R$-modules as objects and $R$-module homomorphisms as morphisms forms a category, which is denoted $R$-mod.

Given $R$-modules $M, N$ such that $N$ is a submodule of $M$, one can define the quotient module $M / N$. This is the $R$-module of equivalence classes of elements of $M$ under the equivalence relation $m \sim n$ if and only if $m-n \in N$. The elements of $M / N$ are written $[m]$ or $m+N .(M / N,+)$ inherits the operation of addition from $(M,+)$, that is, $[m]+[n]:=[m+n]$, and the action of $R$ on $M, r \cdot[m]:=[r \cdot m]$. It follows that these operations are well defined, as $M$ and $N$ are abelian groups, so $N$ is a normal subgroup of $M$. Hence, $M / N$ is indeed an $R$-module.

Consider any $R$-homomorphism $h: M \rightarrow M^{\prime}$, and let $M / N$ be a quotient module with the associated surjective map $j: M \rightarrow M / N$. The quotient module $M / N$ has the universal property that if $N$ is a subset of the kernel of $h$, then there exists exactly one $R$-homomorphism $\tilde{h}$ such that $\tilde{h} \circ j=h$.

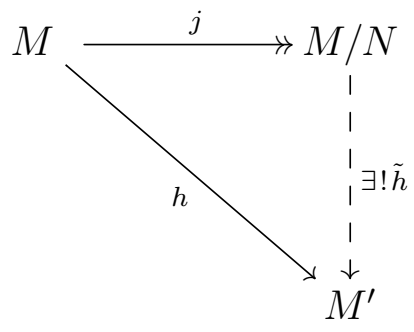


We now define an important quotient module that we consider in this chapter. Let $f: M \rightarrow N$ be an $R$-homomorphism. The co-kernel of an $R$-module homomorphism $f$ is the $R$-module coker $(f):=N / \operatorname{im} f$. The co-kernel of an $R$-homomorphism $f$ is trivial if and only if $f$ is surjective, which is the dual property of the fact that the kernel of $f$ is trivial if and only if $f$ is injective. The co-kernel is always defined since the image of a map $f, \operatorname{im} f$, is always a submodule of its codomain, $N$.

Let $B$ be a subset of $M$. Recall the span of $B, \operatorname{span} B$, is the collection of all linear combinations of $B$ in $M$.

$$
\operatorname{span} B:=\left\{r_{1} \cdot b_{1}+\cdots+r_{k} \cdot b_{k}: r_{i} \in R, b_{i} \in B \text { for all } 1 \leq i \leq k\right\}
$$

span $B$ is a submodule of $M$ as it is closed under addition and scalar multiplication.

Let $M$ be a module. Recall that a set $B$ is linearly independent if the following finite sum

$$
\sum_{i} r_{i} b_{i}=0 \quad \text { where } r_{i} \in R, b_{i} \in B
$$

implies that $r_{i}=0$ for all $i$. The rank of a module $M$ is the cardinality of a maximal independent set. Note that this set doesn't necessarily span $M$.

Given $R$-modules $M, N$, one can define a larger $R$-module called the direct sum of $R$-modules, denoted $M \oplus N$. This $R$-module is the collection of formal sums of elements of $M$ and elements of $N$. Specifically, $M \oplus N=\{m+n: m \in M, n \in N\}$. Addition is as you would expect, $(m+n)+\left(m^{\prime}+n^{\prime}\right):=\left(m+m^{\prime}\right)+\left(n+n^{\prime}\right)$, and scalar multiplication $r \cdot(m+n):=r \cdot m+r \cdot n$.

Suppose $M$ is a module with submodules $N, N^{\prime}$. Suppose that $\operatorname{span}\left(N \cup N^{\prime}\right)=M$ and $N \cap N^{\prime}=\{0\}$. Then, $M=N \oplus N^{\prime}$. 
One can extend the direct sum to a collection of modules $\left\{M_{i}\right\}_{i \in I}$.

$$
\bigoplus_{i \in I} M_{i}:=\left\{\begin{array}{cc}
\sum_{i \in I} r_{i} m_{i}: & m_{i}, r_{i} \in R \text { for } i \in I \\
\text { only finitely many } r_{i} \text { are non-zero }
\end{array}\right\}
$$

For a finite direct sum, one can also write the direct sum as ordered elements, or the Cartesian product, $\left(m_{1}, m_{2}, \ldots, m_{k}\right) \in M_{1} \times \cdots \times M_{k}$. The rank of module $M \oplus N$ is equal to the sum of the $\operatorname{ranks}$ of $M$ and $N, \operatorname{rank}(M \oplus N)=\operatorname{rank} M+\operatorname{rank} N$.

\section{Free modules}

Many nice properties of vector spaces are lost when generalising to modules, and we will look at one particular type of $R$-module that retains some of the useful properties of vector spaces.

A free $R$-module, $M$, is an $R$-module that admits a basis. That is, there exists a set $S \subset M$ so every element $m \in M$ can be written uniquely as a sum

$$
m=\sum_{i} r_{i} s_{i} \quad \text { where } r_{i} \in R, s_{i} \in S
$$

where only finitely many $r_{i}$ are non-zero.

We call the set $S$ a basis of $M$. When a basis $S$ of a module $M$ is finite, the module is called finitely generated. As opposed to vector spaces, not every linearly independent set in $M$ can be augmented to a basis of $M$. In the case that the basis has finite cardinality, every basis of a free module $M$ will have the same cardinality. Therefore, one can define the rank of a finitely generated free module to be the cardinality of a basis $S$ of this free module (note that rank is equal to dimension when referring to vector spaces).

A finitely generated free $R$-module of rank $n$ is isomorphic to the direct sum of $n$ copies of $R$. All modules over a field (that is, vector spaces) are free modules. A 
free module generated by a set $S$ always exists.

Let $S$ be a set, and denote the free module on $S$ over $R$ by $F(S)$, and let $\Phi$ be the inclusion map $\Phi: S \hookrightarrow F(S)$. Free $R$-modules have the universal property that for any $R$-module $G$ and map $g: S \rightarrow G$, there exists exactly one $R$-homomorphism $\tilde{g}: F(S) \rightarrow G$ such that $\tilde{g} \circ \Phi=g$.

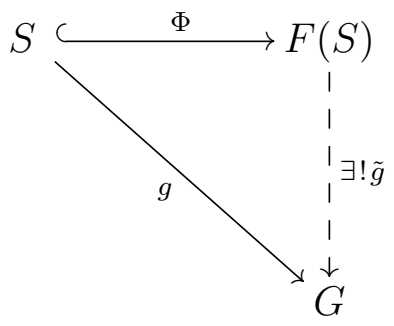

\subsubsection{Tensor product of modules}

This section will be devoted to constructing another $R$-module from other $R$-modules - the tensor product of $R$-modules. Suppose $R$ is commutative. We will first define $a$ tensor product of two $R$-modules, that is describe the universal property we want a tensor product to have. Then we show its existence by constructing it explicitly, and showing that it satisfies the chosen universal property. Subsequently, we can then call it the tensor product, which is unique up to unique isomorphism.

Let $U \times U^{\prime}$ be the cartesian product of the underlying groups of the $R$-modules $U$ and $U^{\prime}$. Let $W$ be an $R$-module. Recall that a $R$-bilinear map is a map $f: U \times U^{\prime} \rightarrow W$ such that $f$ is an $R$-homomorphism in each variable, so $f\left(r \cdot u, u^{\prime}\right)=f\left(u, r \cdot u^{\prime}\right)=$ $r \cdot f\left(u, u^{\prime}\right), f\left(u+v, u^{\prime}\right)=f\left(u, u^{\prime}\right)+f\left(v, u^{\prime}\right)$ and $f\left(u, u^{\prime}+v^{\prime}\right)=f\left(u, u^{\prime}\right)+f\left(u, v^{\prime}\right)$ for every $r \in R, u, v \in U$, and $u^{\prime}, v^{\prime} \in U^{\prime}$.

Let Tens $\left(U, U^{\prime}\right)$ denote a tensor product. We now describe the universal property of a tensor product: there exists a bilinear map $\Psi: U \times U^{\prime} \rightarrow \operatorname{Tens}\left(U, U^{\prime}\right)$ such that for any bilinear map $f: U \times U^{\prime} \rightarrow W$, there is a unique $R$-homomorphism $\tilde{f}: \operatorname{Tens}\left(U, U^{\prime}\right) \rightarrow W$ such that $\tilde{f} \circ \Psi=f$. This implies that a tensor product, should 
it exist, is defined up to unique isomorphism.

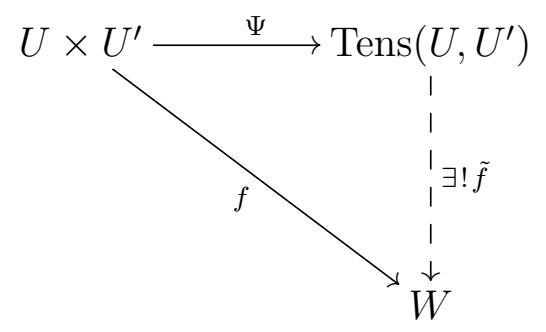

We now construct a tensor product, thereby showing its existence. Let $M$ denote the free $R$-module on the set $U \times U^{\prime}$. We now will construct a submodule $N$ of $M$. Let $N$ be the module generated by elements

$$
\begin{gathered}
\left(u+v, u^{\prime}\right)-\left(u, u^{\prime}\right)-\left(v, u^{\prime}\right), \\
\left(u, u^{\prime}+v^{\prime}\right)-\left(u, u^{\prime}\right)-\left(u, v^{\prime}\right), \\
\left(r \cdot u, u^{\prime}\right)-r \cdot\left(u, u^{\prime}\right), \\
\left(u, r \cdot u^{\prime}\right)-r \cdot\left(u, u^{\prime}\right),
\end{gathered}
$$

where $u, v \in U, u^{\prime}, v^{\prime} \in U^{\prime}$, and $r \in R$.

Then, the quotient module $M / N$ is what we define to be the tensor product of $U$ and $U^{\prime}$, denoted $U \otimes U^{\prime}$, with elements denoted by sums of elements of the form $u \otimes u^{\prime}$. That is, the tensor product of $R$-modules $U$ and $U^{\prime}$ is the $R$-module $U \otimes U^{\prime}$, which is generated by the ordered pairs $\left\{u \otimes u^{\prime}: u \in U, u^{\prime} \in U^{\prime}\right\}$, with the equivalences

$$
\begin{gathered}
(u+v) \otimes\left(u^{\prime}+v^{\prime}\right)=\left(u \otimes u^{\prime}\right)+\left(u \otimes v^{\prime}\right)+\left(v \otimes u^{\prime}\right)+\left(v \otimes v^{\prime}\right), \\
(r \cdot u) \otimes\left(r^{\prime} \cdot u^{\prime}\right)=\left(r r^{\prime}\right) \cdot\left(u \otimes u^{\prime}\right)
\end{gathered}
$$

for all $r, r^{\prime} \in R, u, v \in U$, and $u^{\prime}, v^{\prime} \in U^{\prime}$.

We now check this satisfies the universal property, and hence is well defined. Let $i: U \times U \hookrightarrow M$ be the inclusion map of $U \times U^{\prime}$ to the free module on $U \times U^{\prime}$, 
and let $j: M \rightarrow M / N=U \otimes U^{\prime}$ be the induced quotient map. Define the map $\Psi: U \times U^{\prime} \rightarrow U \otimes U^{\prime}$ to be the composition $j \circ i$. This map $\Psi$ is $R$-bilinear by construction.

Now suppose $f: U \times U^{\prime} \rightarrow W$ is $R$-bilinear. Next we check the existence and uniqueness of an $R$-homomorphism $\tilde{f}: U \otimes U^{\prime} \rightarrow W$ such that $\tilde{f} \circ \Psi=f$. This follows from the universal property of the free module $M$, and the universal property of the quotient module $M / N=U \otimes U^{\prime}$.

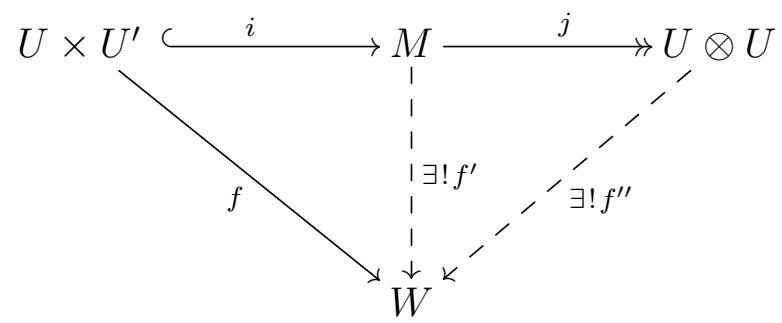

From the left triangle, any $f: U \times U^{\prime} \rightarrow W$, there exists a unique $R$-homomorphism $f^{\prime}$ such that $f^{\prime} \circ i=f$. Since $f$ is bilinear and $f^{\prime}\left(u, u^{\prime}\right)=f\left(u, u^{\prime}\right), f^{\prime}$ is bilinear on the image of $i$, hence we find that $f^{\prime}$ is zero on the generating elements of $N$, therefore $N$ is a subset of the kernel of $f^{\prime}$. Hence from the right triangle, there exists a unique $R$-homomorphism $f^{\prime \prime}$ such that $f^{\prime \prime} \circ j=f^{\prime}$. Combining these equations, with $\Psi=j \circ i$, we have a unique $R$-homomorphism $f^{\prime \prime}$ such that $f^{\prime \prime} \circ \Psi=f$, as required.

To be clear that one is considering the tensor product of modules over the ring $R$, the tensor product is occasionally denoted $\otimes_{R}$. This will often be omitted when the ring is clear from the context.

In the case of finitely generated $R$-modules $U$ and $U^{\prime}$, the rank of the tensor product is the product of the ranks. That is,

$$
\operatorname{rank}\left(U \otimes U^{\prime}\right)=\operatorname{rank} U \cdot \operatorname{rank} U^{\prime}
$$

The tensor product is associative, so one can omit the parentheses of the tensor 
product of more than one $R$-module.

$$
U \otimes\left(U^{\prime} \otimes U^{\prime \prime}\right) \cong\left(U \otimes U^{\prime}\right) \otimes U^{\prime \prime}
$$

Therefore, for $n \in \mathbb{N}$, one can write the $n$th tensor power of a $R$-module $U$ to be $U^{\otimes n}$. By convention, $U^{\otimes 0}=R$.

$$
U^{\otimes n}:=\underbrace{U \otimes U \otimes \cdots \otimes U}_{n}
$$

Also, the tensor product is distributive over direct sums. That is, $U \otimes\left(W \oplus W^{\prime}\right) \cong$ $(U \otimes W) \oplus\left(U \otimes W^{\prime}\right)$.

Example 3.1.1. Consider the tensor product of an $R$-module $W$ and the 1-dimensional free $R$-module, $R$. Then $W \otimes R \cong W \cong R \otimes W$.

We only show $W \otimes R \cong W$, as the other isomorphism follows similarly. Consider the linear map $\Phi: W \rightarrow W \otimes R$, defined by $w \mapsto w \otimes 1$. This is clearly linear as $\Phi(r \cdot w)=(r \cdot w) \otimes 1=r \cdot(w \otimes 1)=r \cdot \Phi(w)$. This map is injective since $\Phi(w)=0$ if and only if $w \otimes 1=0$ if and only if $w=0$. The map $\Phi$ is surjective since if $w \otimes r \in W \otimes R$, then $w \otimes r=w \otimes(r \cdot 1)=(r \cdot w) \otimes 1=\Phi(r \cdot w)$, as required.

The above properties ensure the tensor product is a symmetric monoidal product, which means it has certain nice properties in the category $R$-mod (we employ this without justification). For example, for $R$-homomorphisms $f: M \rightarrow N$ and $f^{\prime}$ : $M^{\prime} \rightarrow N^{\prime}, f \otimes f^{\prime}$ is a well defined $R$-homomorphism from $M \otimes M^{\prime} \rightarrow N \otimes N^{\prime}$, such that $f \otimes f^{\prime}\left(m \otimes m^{\prime}\right)=(f(m)) \otimes\left(f^{\prime}\left(m^{\prime}\right)\right)$. We discuss the concept of symmetric monoidal categories thoroughly in Chapter 7 . 


\subsection{Cochain complexes and cohomology}

\section{A cochain complex of modules}

A cochain complex of $R$-modules is a sequence of $R$-modules $C^{*}:=\left\{C^{i}\right\}_{i \in \mathbb{Z}}$ and $R$ module homomorphisms $\left\{d^{i}: C^{i} \rightarrow C^{i+1}\right\}_{i \in \mathbb{Z}}$ such that the composition $d^{i+1} \circ d^{i}=0$.

$$
\cdots \rightarrow C^{i-1} \stackrel{d^{i-1}}{\longrightarrow} C^{i} \stackrel{d^{i}}{\rightarrow} C^{i+1} \rightarrow \cdots
$$

The requirement that $d^{i} \circ d^{i-1}=0$ is equivalent to im $d^{i-1} \subseteq \operatorname{ker} d^{i}$. The $\left\{C^{i}\right\}_{i \in \mathbb{Z}}$ are called the spaces of the chain complex.

The $R$-homomorphisms $\left\{d^{i}\right\}_{i \in \mathbb{Z}}$ are called the differentials of $C^{*}$. For each $i \in \mathbb{Z}$, the image of $d^{i-1}$ is denoted by $B^{i}\left(C^{*}\right)$, and its elements are known as the $i$-coboundaries of the cochain complex. The kernel of $d^{i}$ is denoted by $Z^{i}\left(C^{*}\right)$, and its elements are called the $i$-cocycles of the cochain complex. The $i$ of $C^{i}$ is its cochain complex degree. We call a cochain complex $C^{*}$ bounded if its spaces are non-trivial for finitely many $i$.

Let $C^{*}$ be a cochain complex with differentials $\left\{d_{C}^{i}\right\}_{i \in \mathbb{Z}}$. A sub-complex of the cochain complex $C^{*}$ is cochain complex $D^{*}$ such that $D^{i}$ is a sub-module of $C^{i}$ for every $i$, and the $i$ th differential of $D^{*}$ is the restriction of $d_{C}^{i}$ to $D^{i}$, denoted $d_{D}^{i}$. Now let $D^{*}$ be a sub-complex of a cochain complex $C^{*}$. A quotient of the cochain complex $C^{*}$ with respect to $D^{*}$ is the cochain complex $(C / D)^{*}$ which has spaces $C^{i} / D^{i}$ in degree $i$. Its differentials are the induced differentials of $C^{*}$, which are well defined.

Consider cochain complexes $C^{*}, D^{*}$ with respective differentials $d_{C}^{*}, d_{D}^{*}$. The direct sum of $C^{*}$ and $D^{*}$, denoted $C^{*} \oplus D^{*}$, is the cochain complex with spaces $C^{i} \oplus D^{i}$, and differentials $d^{i}$ defined by $d^{i}(c, d)=\left(d_{C}^{i} c, d_{D}^{i} d\right)$ for $c \in C^{i}, d \in D^{i}$.

For a cochain complex $C^{*}$, the height shift operator $\cdot[s]$ with $s \in \mathbb{Z}$ defines a new 
chain complex $C^{*}[s]$ by

$$
C^{n}[s]:=C^{n-s}
$$

The differentials of $C^{*}[s]$ are also shifted. That is, the $n$th differential of $C^{*}[s]$ is the $(n-s)$ th differential of $C^{*}$.

\section{Definition 3.2.1. Cohomology of a cochain complex}

The nth cohomology module of a cochain complex $C^{*}$ is the module defined by the quotient

$$
H^{n}\left(C^{*}\right):=Z^{n}\left(C^{*}\right) / B^{n}\left(C^{*}\right)
$$

This is well defined since it is required that $B^{n}\left(C^{*}\right) \subseteq Z^{n}\left(C^{*}\right)$ for every $n$ by definition of a chain complex, and in fact $B^{n}\left(C^{*}\right)$ is a submodule of $Z^{n}\left(C^{*}\right)$, by the properties of homomorphisms.

Suppose $C^{*}$ is a chain complex such that $H^{n}\left(C^{*}\right)=0$. Then one says $C^{*}$ is exact at $C^{n}$. If $H^{n}\left(C^{*}\right)=0$ for every $n$, then $C^{*}$ is called an exact sequence, or we say it has zero homology. Also, a chain complex $C^{*}$ is called acyclic if it has zero homology.

Note that the shift operator $\cdot[s]$ on a cochain complex extends to the homology of the complex, which is clear since the differentials are also shifted.

$$
H^{n}\left(C^{*}[s]\right)=H^{n-s}\left(C^{*}\right)
$$

Example 3.2.2. Consider the following cochain complex $M^{*}$ of $\mathbb{Z}$-modules.

$$
M^{i}= \begin{cases}\mathbb{Z} / 8 \mathbb{Z} & : i \geq 0 \\ 0 & : i<0\end{cases}
$$


With the following differentials.

$$
d^{i}=\left\{\begin{array}{lll}
4 & \text { so } d^{i}(x)=4 x & : i \geq 0 \\
0 & \text { so } d^{i}(x)=0 & : i<0
\end{array}\right.
$$

Then the cochain complex $M^{*}$ is as follows.

$$
\cdots \longrightarrow 0 \stackrel{0}{\longrightarrow} 0 \stackrel{0}{\longrightarrow} \mathbb{Z} / 8 \mathbb{Z} \stackrel{4}{\longrightarrow} \mathbb{Z} / 8 \mathbb{Z} \stackrel{4}{\longrightarrow} \mathbb{Z} / 8 \mathbb{Z} \longrightarrow \cdots
$$

This is a cochain complex as for degrees $\leq 0,0 \circ 0=0$ and $4 \circ 4=0$, and for degrees $>0, \operatorname{im} 4=\{0,4\}$ and ker $4=\{0,2,4,6\}$. Therefore $\operatorname{im} 4 \subseteq$ ker 4 as required.

The cohomology modules for this complex are:

$$
H^{i}\left(M^{*}\right) \cong \begin{cases}\mathbb{Z} / 2 \mathbb{Z} & : i>0 \\ \mathbb{Z} / 4 \mathbb{Z} & : i=0 \\ 0 & : i<0\end{cases}
$$

Since, for $i<0, H^{i}\left(M^{*}\right)=0 / 0=0$. For $i=0, H^{i}\left(M^{*}\right)=\{0,2,4,6\} /\{0\} \cong \mathbb{Z} / 4 \mathbb{Z}$. Lastly, for $i>0, H^{i}\left(M^{*}\right)=\{0,2,4,6\} /\{0,4\} \cong \mathbb{Z} / 2 \mathbb{Z}$.

\section{Cochain complex maps}

Let $C^{*}$ and $D^{*}$ be cochain complexes with differentials $d_{C}^{*}$ and $d_{D}^{*}$ respectively. A cochain complex map $\left\{f^{*}: C^{*} \rightarrow D^{*}\right\}$ is a collection of $R$-homomorphisms $\left\{f^{n}\right.$ : $\left.C^{n} \rightarrow D^{n}\right\}_{n \in \mathbb{Z}}$ that commute with the differentials of $C^{*}$ and $D^{*}$. So, there is a commuting "ladder" diagram:

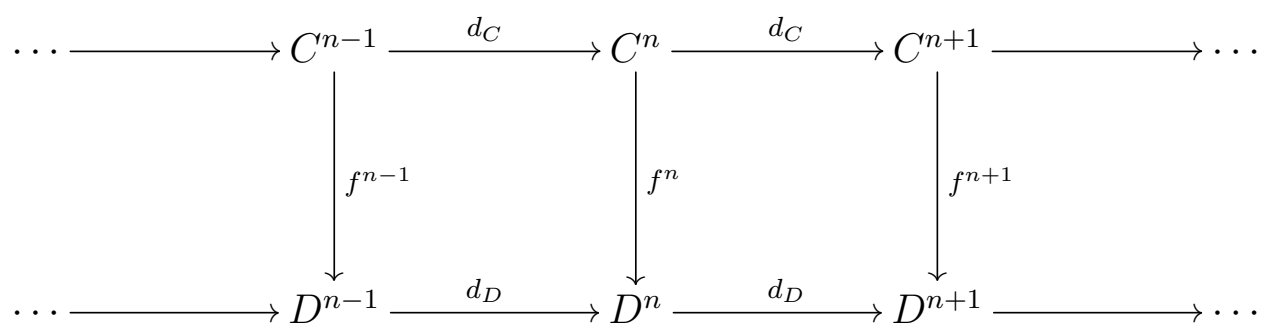


In other words, $d_{D} \circ f^{n}=f^{n+1} \circ d_{C}$ for every $n$. For clarity, the composition symbol "०" will sometimes be omitted in the following working.

Chain complexes of $R$-modules with chain complex maps form a category denoted $\mathbf{C h}(R$-mod $)$. The identity chain complex map is clearly the chain map which is the collection of identity maps at each degree $n$, and is written $\operatorname{id}_{C^{*}}$. The zero chain complex map is the map which is zero for every degree $n$, and is denoted $0^{*}$.

A cochain complex map $f^{*}: C^{*} \rightarrow D^{*}$ induces an $R$-homomorphism $\overline{f^{n}}: H^{n}\left(C^{*}\right) \rightarrow$ $H^{n}\left(D^{*}\right)$ on cohomology for every $n$. To show this induced map is well defined, we first show that $f^{*}$ sends cocycles to cocycles. If $c \in \operatorname{ker} d_{C}$, then since $f^{*}$ is a cochain map, $d_{D} f^{n}(c)=f^{n+1} d_{C}(c)=0$, which implies $f^{n}(c)$ is a cocycle of $D^{n}$. Therefore, $f^{n}\left(Z^{n}\left(C^{*}\right)\right) \subseteq Z^{n}\left(D^{*}\right)$.

Next we show that the induced map is well defined under the quotient by the boundary $B^{n}\left(C^{*}\right)$. Suppose $c, c^{\prime}$ represent the same element in $H^{n}\left(C^{*}\right)$, so $c-c^{\prime} \in B^{n}\left(C^{*}\right)$. Then $c-c^{\prime}=d_{C} b$ for some $b \in C^{n-1}$. Therefore, $f^{n}\left(c-c^{\prime}\right)=f^{n} d_{C}(b)=d_{D} f^{n-1}(b)$, so it follows that $f^{n}(c)-f^{n}\left(c^{\prime}\right) \in B^{n}\left(D^{*}\right)$. This shows that $f^{n}(c)$ and $f^{n}\left(c^{\prime}\right)$ represent the same equivalence class in $H^{n}\left(D^{*}\right)$, as required.

\section{Definition 3.2.3. Quasi-isomorphism}

A chain complex map is called a quasi-isomorphism if the induced maps are isomorphisms on the cohomology modules for every $n$. Hence, quasi-isomorphisms form an equivalence relation on chain complexes.

Next we state a proposition that states $H^{n}$ has nice properties, that it, it is a functor.

Proposition 3.2.4. The nth cohomology module $H^{n}$ is a functor from $\mathbf{C h}(R$-mod) to $R$-mod. More generally, for an abelian category $\mathcal{A}, H^{n}$ is a functor from $\operatorname{Ch}(\mathcal{A})$ to $\mathcal{A}$. 
This section, as well as the rest of this chapter, can be generalised to abelian categories, which will not be explicitly described here, but is done in [14]. Moreover, one can show that if $\mathcal{A}$ is an abelian category, $\operatorname{Ch}(\mathcal{A})$ is also an abelian category. Hence, many of the previous discussions can be extended to homology of cochain complexes, of which we will discuss some details now.

In the next proposition, we explain the kernel and co-kernel of a chain complex of $R$-modules are in the category $\mathbf{C h}(R$-mod $)$. The kernel and co-kernel of a chain map $f^{*}$ are denoted ker $f^{*}$ and coker $f^{*}$ respectively. There is slightly confusing terminology, as both the chain complex ker $f^{*}$ and the inclusion map ker $f^{*} \hookrightarrow C^{*}$ are denoted ker $f^{*}$. Similarly, both the chain complex coker $f^{*}$ and the inclusion map $D^{*} \rightarrow$ coker $f^{*}$ are denoted coker $f^{*}$.

Proposition 3.2.5. Let $f^{*}: C^{*} \rightarrow D^{*}$ be a chain complex map of $R$-modules.

The kernel of a chain complex map $f^{*}$ in the category $\mathbf{C h}(R$-mod $)$, is the following chain complex of the kernels of each $f^{i}$, where the differentials $d_{\mathrm{ker} f^{*}}^{*}$ are the restrictions $\left.d^{i}\right|_{\text {ker } f^{*}}$.

$$
\cdots \longrightarrow \operatorname{ker} f^{i-1} \stackrel{d_{\text {ker } f}^{i-1}}{\longrightarrow} \operatorname{ker} f^{i} \stackrel{d_{\text {ker } f}^{i}}{\longrightarrow} \operatorname{ker} f^{i+1} \longrightarrow \cdots
$$

The co-kernel of a chain complex map $f^{*}$ in the category $\mathbf{C h}(R$-mod $)$, is the following chain complex of the co-kernels of each $f^{i}$, where the differentials $d_{\text {coker } f^{*}}^{*}$ are the induced maps on quotients $d^{i}$ o coker $f^{i}$.

$$
\cdots \longrightarrow \operatorname{cokerf}^{i-1} \stackrel{d_{\text {cokerf }}^{i-1}}{\longrightarrow} \operatorname{coker} f^{i} \stackrel{d_{\text {cokerf }}^{i}}{\longrightarrow} \operatorname{cokerf}^{i+1} \longrightarrow \cdots
$$

Proof. It is clear that $\operatorname{ker} f^{*}$ and coker $f^{*}$ have the desired properties of a kernel and co-kernel in $\mathbf{C h}(R$-mod $)$. That is, ker $f^{*}$ has the universal property of a map such that $f^{*} \circ\left(\operatorname{ker} f^{*}\right)=0^{*}$, and coker $f^{*}$ has the universal property of a map such that $\left(\operatorname{coker} f^{*}\right) \circ f^{*}=0^{*}$.

We first check that $\operatorname{ker} f^{*}$ and coker $f^{*}$ as defined above are in fact well defined 
sequences. Then we check they form chain complexes with their induced differentials, and finally that their respective vertical maps $\left(\left\{\operatorname{ker} f^{i}\right\}_{i \in \mathbb{Z}}\right.$ and $\left.\left\{\operatorname{coker} f^{i}\right\}_{i \in \mathbb{Z}}\right)$ are in fact chain maps.

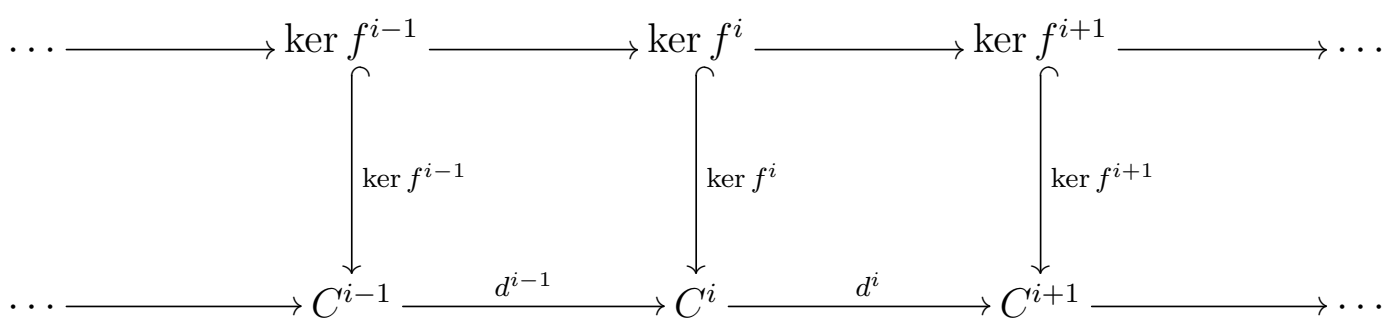

The top row of the above diagram is what we want to show is the kernel space of the chain map $f^{*}$. The vertical maps is what we want to show is the inclusion kernel map of $f^{*}$, also denoted ker $f^{*}$. First, we check that $\left.d^{i}\right|_{k e r f^{i}}\left(\operatorname{ker} f^{i}\right) \subseteq \operatorname{ker} f^{i+1}$. Take some $c \in \operatorname{ker} f^{i}$. Then $\left.f^{i+1} d^{i}\right|_{k e r f^{i}}(c)=f^{i+1} d^{i}(c)=d^{i} f^{i}(c)=0$. Hence, $c \in \operatorname{ker} f^{i+1}$.

Next, we check $\left.\left.d^{i+1}\right|_{\operatorname{ker} f^{i+1}} d^{i}\right|_{\operatorname{ker} f^{i}}=0$. Take $c \in \operatorname{ker} f^{i}$, then $\left.\left.d^{i+1}\right|_{\operatorname{ker} f^{i+1}} d^{i}\right|_{\operatorname{ker} f^{i}}(c)=$ $\left.d^{i+1}\right|_{\operatorname{ker} f^{i+1}} d^{i}(c)=d^{i+1} d^{i}(c)=0$, as required. It follows that $d^{i} \operatorname{ker} f^{i}=\left.\operatorname{ker} f^{i+1} d^{i}\right|_{\operatorname{ker} f^{i}}$ from the construction of the kernel maps. Explicitly, take some $c \in \operatorname{ker} f^{i}$. Then $d^{i} \operatorname{ker} f^{i}(c)=d^{i}(c)=\operatorname{ker} f^{i+1} d^{i}(c)$. This shows that ker $f^{*}$ is the collection of kernel maps of each component of the chain map, or $\left\{\operatorname{ker} f^{i}\right\}_{i \in \mathbb{Z}}$, as required.

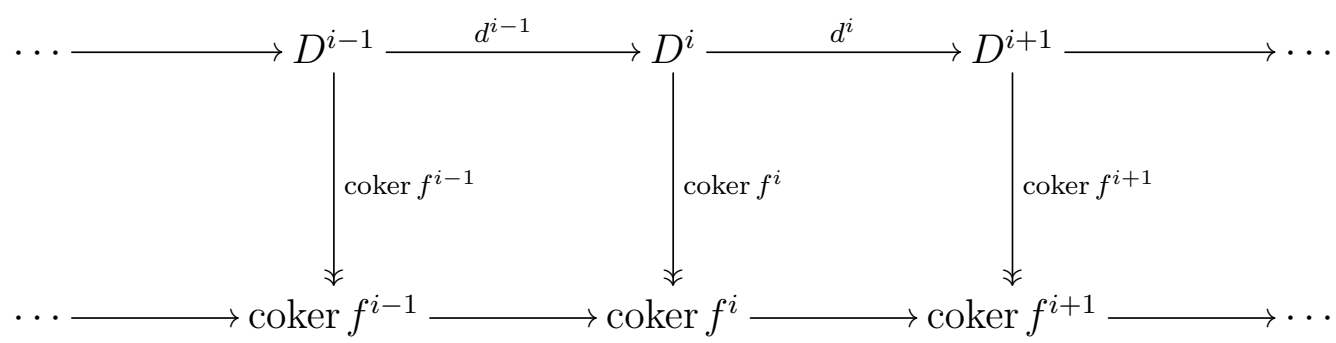

The bottom row of the above diagram is what we want to show is the co-kernel space of the chain map $f^{*}$. The vertical maps is what we want to show is the quotient cokernel map of $f^{*}$, also denoted coker $f^{*}$. First, we check that $\overline{d^{i}}$ : coker $f^{i} \rightarrow \operatorname{coker} f^{i+1}$ is well defined. Take some $c_{1}, c_{2} \in D^{i}$ such that $c_{1}-c_{2}=f^{i}\left(c^{\prime}\right)$ for some $c^{\prime} \in C^{i}$. Hence 
$c_{1}, c_{2}$ represent the same element in coker $f^{i}$. Then $\overline{d^{i}}\left(\left[c_{1}\right]\right)-\overline{d^{i}}\left(\left[c_{2}\right]\right)=d^{i}\left(c_{1}\right)-d^{i}\left(c_{2}\right)=$ $d^{i} f^{i}\left(c^{\prime}\right)=f^{i+1} d^{i}\left(c^{\prime}\right)$. So $\overline{d^{i}}\left(\left[c_{1}\right]\right)-\overline{d^{i}}\left(\left[c_{2}\right]\right) \in \operatorname{im} f^{i+1}$, which shows $\overline{d^{i}}$ is well defined.

Next, we check $\overline{d^{i+1} d^{i}}=0$. Take $[c] \in \operatorname{coker} f^{i}$, then $\overline{d^{i+1} d^{i}}([c])=\overline{d^{i+1}}\left(\left[d^{i}(c)\right]\right)=$ $\left[d^{i+1} d^{i}(c)\right]=[0]$, as required. It follows that $\overline{d^{i}}$ coker $f^{i}=$ coker $f^{i+1} d^{i}$ from the construction of the co-kernel maps. Explicitly, take some $c \in C^{i}$. Then $\overline{d^{i}}$ coker $f^{i}(c)=$ $d^{i}([c])=\left[d^{i}(c)\right]=$ coker $f^{i+1}\left(d^{i}(c)\right)$. This shows that coker $f^{*}$ is the collection of cokernel maps of each component of the chain map, or $\left\{\text { coker } f^{i}\right\}_{i \in \mathbb{Z}}$, as required.

Hence, we have shown that the chain complexes ker $f$ and coker $f$ are indeed cochain complexes with induced differentials.

\subsubsection{Euler characteristic}

The Euler characteristic is a well known topological invariant, which is the alternating sum of the number of $n$-cells. For example, for a 2-dimensional surface, with $V$ vertices, $E$ edges, and $F$ faces, the Euler characteristic is $V-E+F$, 44. Hence, as homology is a topological invariant, the Euler characteristic is an invariant of homology, and is defined as follows.

\section{Definition 3.2.6. Euler characteristic}

Suppose $C^{*}$ is a bounded cochain complex which has finitely generated spaces. The Euler characterisitic, $\chi$, of $C^{*}$ is the alternating sum of the ranks of its cohomology modules, $H^{k}\left(C^{*}\right)$.

$$
\chi\left(C^{*}\right):=\sum_{k \in \mathbb{Z}}(-1)^{k} \operatorname{dim} H^{k}\left(C^{*}\right)
$$

Proposition 3.2.7. The Euler Characteristic is an invariant of cohomology.

Proof. If $H^{n}\left(B^{*}\right) \cong H^{n}\left(C^{*}\right)$ for every $n$, then the dimension of the homology group are the same for every $n \in \mathbb{Z}$. It follows that the sum of the dimensions over all homology groups are equal, so $\chi\left(B^{*}\right)=\chi\left(C^{*}\right)$.

In this thesis, the above is important as the Jones polynomial is the graded Euler characteristic of the Khovanov homology. Hence, what Khovanov has done is replaced 
a polynomial with a cochain complex which has the graded Euler characteristic of the original polynomial. This is an example of "categorification". A typical example of categorification is replacing the cardinality of a finite set (which is an invariant of finite sets), with the category of the sets themselves. The Khovanov homology has also inspired other categorifications of polynomial knot invariants, as discussed in [13].

\subsubsection{Exact sequences}

A sequence $C^{*}$ is called exact at $C^{n}$ if $H^{n}\left(C^{*}\right)=0$. This is equivalent to $\operatorname{im} d^{n-1}=$ ker $d^{n}$.

Let $A, B$, and $C$ be $R$-modules. A short exact sequence is a sequence

$$
0 \rightarrow A \stackrel{f}{\rightarrow} B \stackrel{g}{\rightarrow} C \rightarrow 0
$$

such that the sequence is exact at every module. Such a sequence is exact if and only if $f$ is injective ( $\operatorname{ker} f=0), g$ is surjective (coker $g=0$ ), and $\operatorname{im} f=\operatorname{ker} g$.

We claim that a short sequence of chain complexes, $0 \rightarrow A^{*} \stackrel{f^{*}}{\rightarrow} B^{*} \stackrel{g^{*}}{\rightarrow} C^{*} \rightarrow 0$ is exact in $\mathbf{C h}(R$-mod $)$ if and only if the sequence $0 \rightarrow A^{n} \stackrel{f^{n}}{\longrightarrow} B^{n} \stackrel{g^{n}}{\longrightarrow} C^{n} \rightarrow 0$ is exact for every $n$. The forward direction is clear. The converse follows because ker $f^{n}=0$ for every $n$, hence $0^{*}$ forms the kernel of $f^{*}$, and similarly the cokernel of $g^{n}$ for all $n$ forms coker $g^{*}$. We know that $\operatorname{im} f^{n}=\operatorname{ker} g^{n}$ are subspaces of $B^{n}$ for every $n$, and because $\operatorname{ker} g^{n}$ forms a subcomplex $\operatorname{ker} g^{*}$ of $B^{*}, \operatorname{im} f^{*}=\operatorname{ker} g^{*}$ as subcomplexes.

There is a very useful theorem in homology theory when considering short exact sequences of chain complexes.

Theorem 3.2.8. Consider the short exact sequence of chain complexes:

$$
0 \rightarrow A^{*} \stackrel{f^{*}}{\rightarrow} B^{*} \stackrel{g^{*}}{\rightarrow} C^{*} \rightarrow 0
$$


There exist natural R-homomorphisms $\partial^{n}: H^{n}(C) \rightarrow H^{n+1}(A)$ such that the homology groups under the induced maps form a long exact sequence as follows.

$\cdots \rightarrow H^{n-1}\left(C^{*}\right) \stackrel{\partial^{n-1}}{\longrightarrow} H^{n}\left(A^{*}\right) \stackrel{\overline{f^{n}}}{\longrightarrow} H^{n}\left(B^{*}\right) \stackrel{\overline{g^{n}}}{\longrightarrow} H^{n}\left(C^{*}\right) \stackrel{\partial^{n}}{\longrightarrow} H^{n+1}\left(A^{*}\right) \rightarrow \cdots$

The proof of this theorem uses the Snake Lemma, which we will prove below.

\section{Lemma 3.2.9. Snake Lemma}

Consider the following commuting diagram.

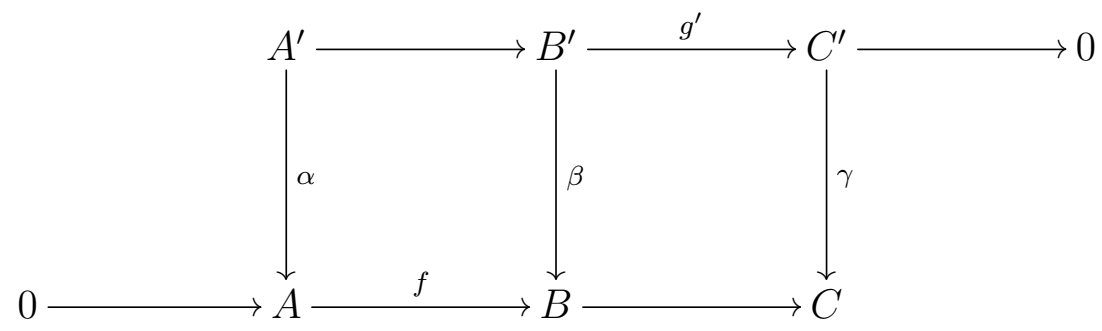

If the rows are exact, then the following is an exact sequence.

$$
\operatorname{ker} \alpha \rightarrow \operatorname{ker} \beta \rightarrow \operatorname{ker} \gamma \stackrel{\partial}{\rightarrow} \operatorname{coker} \alpha \rightarrow \operatorname{coker} \beta \rightarrow \operatorname{coker} \gamma
$$

Where the map $\partial$ is defined as

$$
\partial\left(c^{\prime}\right):=f^{-1} \circ \beta \circ\left(g^{\prime}\right)^{-1}\left(c^{\prime}\right) \quad c^{\prime} \in \operatorname{ker} \gamma
$$

Proof. We begin by showing the sequence is well defined. Consider the following commuting diagram with, by the assumption, exact second and third rows. 


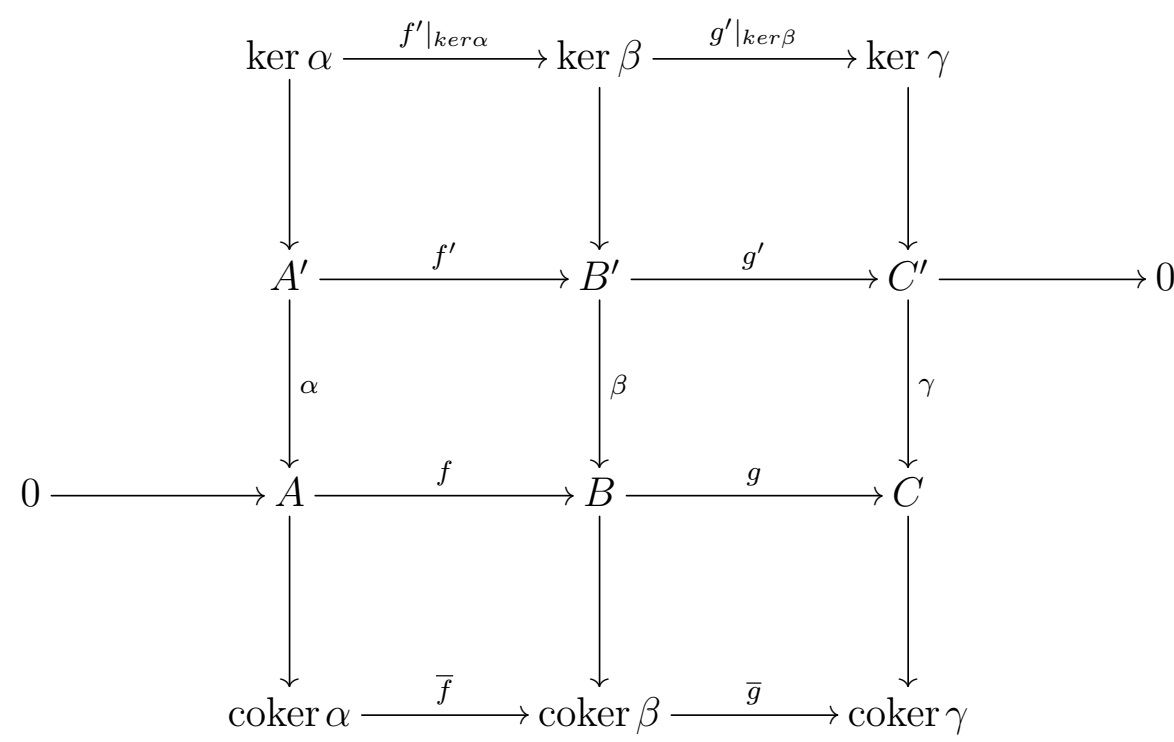

If we consider the middle two rows as chain complexes with chain maps $\alpha, \beta, \gamma$, the top row is the kernel complex and the bottom row is the co-kernel complex with respective induced maps. Hence it is indeed a commuting diagram.

We claim the map $\partial: \operatorname{ker} \gamma \rightarrow \operatorname{coker} \alpha$ defined by $\partial\left(c^{\prime}\right):=f^{-1} \beta g^{\prime-1}\left(c^{\prime}\right)$ is well defined. By exactness of the second row, $g^{\prime}$ is surjective, so $g^{\prime-1}\left(c^{\prime}\right)$ exists. Also by exactness of the third row, we know that $\operatorname{im} f=\operatorname{ker} g$. By the commutativity of the right square, $g \beta g^{\prime-1}\left(c^{\prime}\right)=\gamma g^{\prime} g^{-1}\left(c^{\prime}\right)=\gamma\left(c^{\prime}\right)=0$, so $\beta g^{\prime-1}\left(c^{\prime}\right) \in \operatorname{ker} g=\operatorname{im} f$, and by the injectivity of $f$, there exists a unique element $a \in A$ such that $f(a)=\beta g^{-1}\left(c^{\prime}\right)$. So, the equivalence class represented by $a$ in coker $\alpha$ is the image element of $c^{\prime}$ via $\partial$.

Therefore, to check that $\partial$ is well defined, we need to ensure that the function is not affected by the choice of $b^{\prime} \in B^{\prime}$ such that $g^{\prime}\left(b^{\prime}\right)=c^{\prime}$. Suppose $g^{\prime}\left(b^{\prime}\right)=g^{\prime}\left(b^{\prime \prime}\right)=c^{\prime}$. It suffices to show that $f^{-1} \beta\left(b^{\prime}-b^{\prime \prime}\right) \in \operatorname{im} \alpha$, and therefore $f^{-1} \beta\left(b^{\prime}\right)$ and $f^{-1} \beta(b)$ represent the same equivalence class in coker $\alpha$. By exactness of the second row, $\operatorname{im} f^{\prime}=\operatorname{ker} g^{\prime}$, so since $g^{\prime}\left(b^{\prime}-b^{\prime \prime}\right)=0$, there exists $a^{\prime} \in A^{\prime}$ such that $f^{\prime}\left(a^{\prime}\right)=b^{\prime}-b^{\prime \prime}$. By commutativity of the left square, $f^{-1} \beta\left(b^{\prime}-b^{\prime \prime}\right)=f^{-1} \beta f^{\prime}\left(a^{\prime}\right)=\alpha\left(a^{\prime}\right) \in \operatorname{im} \alpha$, which shows that $f^{-1} \beta\left(b^{\prime}\right)$ and $f^{-1} \beta\left(b^{\prime \prime}\right)$ represent the same equivalence class in coker $\alpha$, as required. 
Finally, we show the sequence is exact, by showing exactness in four places.

(i) $\left.\operatorname{im} f^{\prime}\right|_{\operatorname{ker} \alpha}=\left.\operatorname{ker} g^{\prime}\right|_{\operatorname{ker} \beta}$

This follows by the commutativity of the top two squares and exactness of the second row. We know already that the induced maps $\left.\left.g^{\prime}\right|_{\operatorname{ker} \beta} f^{\prime}\right|_{\operatorname{ker} \alpha}=0$ since the top row is a chain complex. The $\operatorname{kernel} \operatorname{ker}\left(\left.g^{\prime}\right|_{\operatorname{ker} \beta}\right)$ is contained in the image $\operatorname{im}\left(\left.f^{\prime}\right|_{\operatorname{ker} \alpha}\right)$, since suppose $g^{\prime}\left(b^{\prime}\right)=0$ for some $b^{\prime} \in \operatorname{ker} \beta$. By exactness at $B^{\prime}$, there is an $a^{\prime} \in A^{\prime}$ such that $f^{\prime}\left(a^{\prime}\right)=b^{\prime}$. Therefore by commutativity of the left square, $f \alpha\left(a^{\prime}\right)=\beta f^{\prime}\left(a^{\prime}\right)=\beta\left(b^{\prime}\right)=0$, and since $f$ is injective, $\alpha\left(a^{\prime}\right)=0$. So there is an $a^{\prime} \in \operatorname{ker} \alpha$ such that $\left.f^{\prime}\right|_{\operatorname{ker} \alpha}\left(a^{\prime}\right)=b^{\prime}$.

(ii) $\left.\operatorname{im} g^{\prime}\right|_{\operatorname{ker} \beta}=\operatorname{ker} \partial$

For $\left.\operatorname{im} g^{\prime}\right|_{\operatorname{ker} \beta} \subseteq \operatorname{ker} \partial$, suppose there exists $b^{\prime} \in \operatorname{ker} \beta$ such that $g^{\prime}\left(b^{\prime}\right)=c^{\prime}$. Then $\partial\left(c^{\prime}\right)=f^{-1} \beta g^{\prime-1} g^{\prime}\left(b^{\prime}\right)=f^{-1} \beta\left(b^{\prime}\right)=f^{-1}(0)=0$. ker $\left.\partial \subseteq \operatorname{im} g^{\prime}\right|_{\operatorname{ker} \beta}$ : let $c^{\prime}$ such that $\partial\left(c^{\prime}\right)=f^{-1} \beta g^{-1}\left(c^{\prime}\right) \in \operatorname{im} \alpha$. Then $f^{-1} \beta g^{-1}\left(c^{\prime}\right)=\alpha\left(a^{\prime}\right)$ for some $a^{\prime} \in A^{\prime}$, and therefore $\beta g^{\prime-1}\left(c^{\prime}\right)=f \alpha\left(a^{\prime}\right)=\beta f^{\prime}\left(a^{\prime}\right)$. Choose $b^{\prime}=g^{\prime-1}\left(c^{\prime}\right)-f^{\prime}\left(a^{\prime}\right)$, which is in $\operatorname{ker} \beta$ since $\beta g^{\prime-1}\left(c^{\prime}\right)-\beta f^{\prime}\left(a^{\prime}\right)=0$ and $g^{\prime}\left(b^{\prime}\right)=g^{\prime} g^{\prime-1}\left(c^{\prime}\right)-g^{\prime} f^{\prime}\left(a^{\prime}\right)=c^{\prime}-0=$ $c^{\prime}$, which shows $\left.c^{\prime} \in \operatorname{im} g^{\prime}\right|_{\operatorname{ker} \beta}$, as required.

(iii) $\operatorname{im} \partial=\operatorname{ker} \bar{f}$

Clearly $\operatorname{im} \partial \subseteq \operatorname{ker} \bar{f}$, since $\bar{f} \partial\left(c^{\prime}\right)=\bar{f} f^{-1} \beta g^{\prime-1}\left(c^{\prime}\right)=\beta g^{-1}\left(c^{\prime}\right) \in \operatorname{im} \beta$, which is trivial in coker $\beta$. $\operatorname{ker} \bar{f} \subseteq \operatorname{im} \partial$ since if $f(a) \in \operatorname{im} \beta$, there is $b^{\prime} \in B^{\prime}$ such that $\beta\left(b^{\prime}\right)=f(a)$ and $\gamma g^{\prime}\left(b^{\prime}\right)=g \beta\left(b^{\prime}\right)=g f(a)=0$, and so can choose $c^{\prime}:=g^{\prime}\left(b^{\prime}\right) \in \operatorname{ker} \alpha$ so that $\partial\left(c^{\prime}\right)=a+\operatorname{im} \gamma \in \operatorname{coker} \alpha$, as required.

(iv) $\operatorname{im} \bar{f}=\operatorname{ker} \bar{g}$

We know $\bar{g} \bar{f}=0$ since the bottom row is a chain complex. Suppose $\bar{g}(b+\operatorname{im} \beta)=$ $\operatorname{im} \gamma$. Then there is $b^{\prime} \in B^{\prime}$ such that $g \beta\left(b^{\prime}\right)=g(b)$, since $g^{\prime}$ is surjective. Therefore, $g\left(b-\beta\left(b^{\prime}\right)\right)=0$, and so as the third row is exact, there exists $a \in A$ such that $f(a)=b-\beta\left(b^{\prime}\right)$. So, $\bar{f}(a+\operatorname{im} \alpha)=b-\beta\left(b^{\prime}\right)+\operatorname{im} \beta=b+\operatorname{im} \beta$, as required. 
Proof of Theorem 3.2.8. Consider the short exact sequence of cochain complexes:

$$
0 \rightarrow A^{*} \stackrel{f^{*}}{\longrightarrow} B^{*} \stackrel{g^{*}}{\longrightarrow} C^{*} \rightarrow 0
$$

with differentials $\left\{\alpha^{n}: A^{n} \rightarrow A^{n+1}\right\}_{n \in \mathbb{Z}},\left\{\beta^{n}: B^{n} \rightarrow B^{n+1}\right\}_{n \in \mathbb{Z}}$, and $\left\{\gamma^{n}: C^{n} \rightarrow\right.$ $\left.C^{n+1}\right\}_{n \in \mathbb{Z}}$. We consider a segment of this short exact sequence with appropriate kernels in the top row and co-kernels in the bottom row as in the proof of the Snake Lemma:

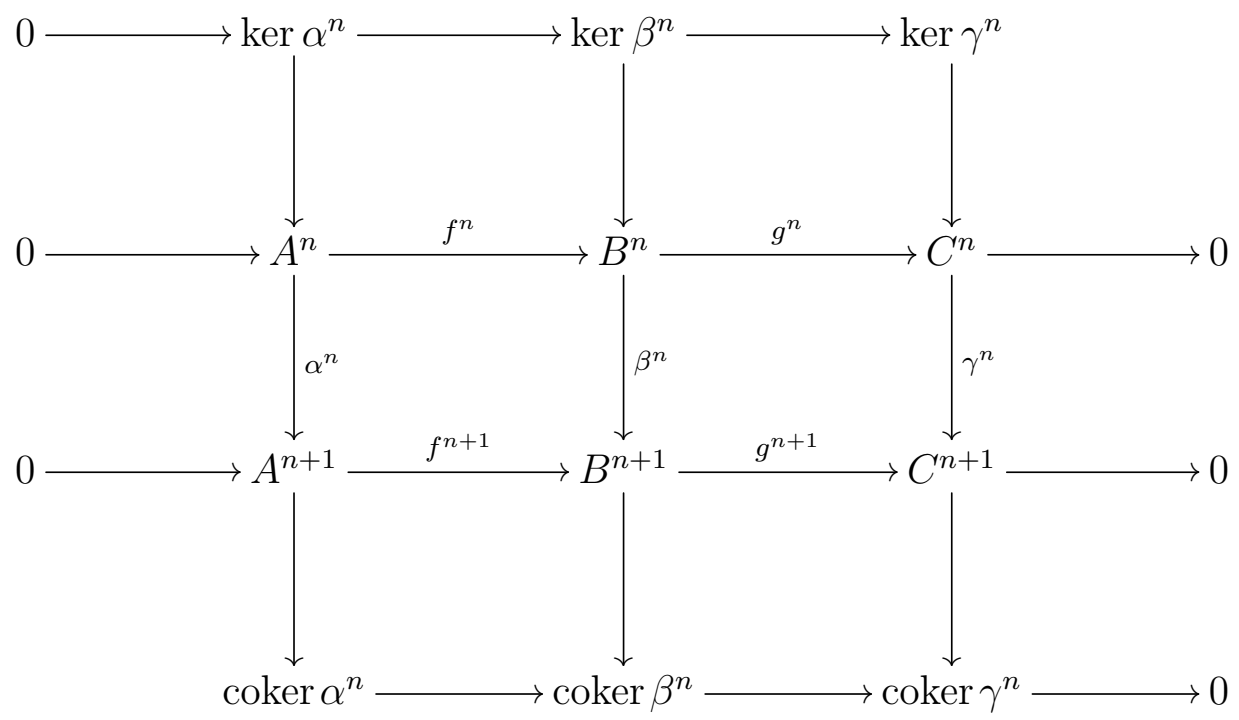

We know that the second and third rows are exact from the discussion at the beginning of this section. Therefore, we apply the discussion in the proof of the Snake Lemma to know that this diagram commutes, and also that the top and bottom rows are exact except at ker $\alpha^{n}$ and coker $\gamma^{n}$. Since $\operatorname{ker} \alpha^{n} \rightarrow \operatorname{ker} \beta^{n}$ is induced from $f^{n}$, it follow from the injectivity of $f^{n}$, and similarly for coker $\gamma^{n}$. Therefore, we now have shown that this is a commutative diagram with exact rows and columns.

Consider the natural induced map $\tilde{\alpha}^{n}: A^{n} / \operatorname{im} \alpha^{n-1} \rightarrow \operatorname{ker} \alpha^{n+1}$ of $\alpha^{n}: A^{n} \rightarrow$ $\operatorname{ker} \alpha^{n+1}$. Since $\operatorname{im} \alpha^{n-1} \subseteq \operatorname{ker} \alpha^{n}$, this map exists and is unique by the universal property of the quotient space $A^{n} / \operatorname{im} \alpha^{n-1}$. 
Note that $[a] \in A^{n} / \operatorname{im} \alpha^{n-1}$ is in the kernel of $\tilde{\alpha}^{n}$ if and only if $a \in \operatorname{ker} \alpha^{n}$, and since $\operatorname{im} \alpha^{n-1} \subseteq \operatorname{ker} \alpha^{n}$, then $[a] \in \operatorname{ker} \alpha^{n} / \operatorname{im} \alpha^{n-1}$. This implies that $\operatorname{ker} \tilde{\alpha}^{n}=H^{n}\left(A^{*}\right)$. The image of $\tilde{\alpha}^{n}$ is the same as the image of $\alpha^{n}$, and so coker $\tilde{\alpha}^{n}=H^{n+1}\left(A^{*}\right)$. The homology groups of $B^{*}$ and $C^{*}$ are formed similarly from the kernels and co-kernels of $\tilde{\beta^{n}}$ and $\tilde{\gamma^{n}}$.

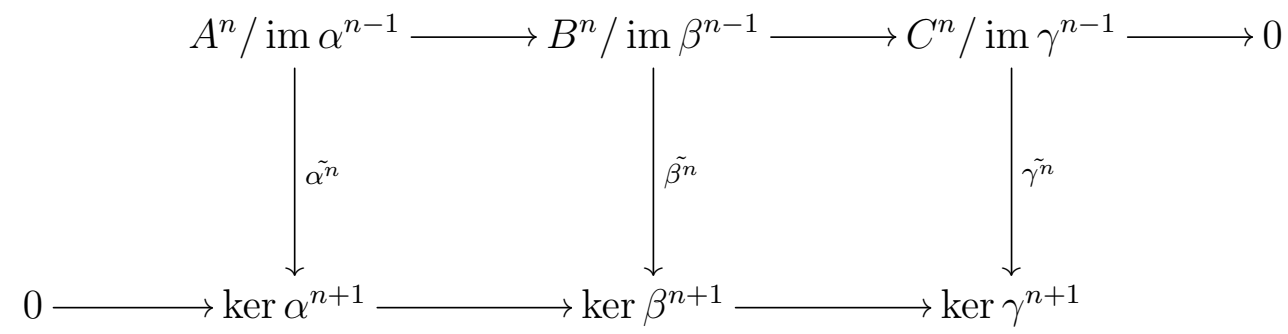

Therefore, by the Snake Lemma, there is an exact map $\partial^{n}$ that forms an exact sequence as follows. All other maps are induced maps on homology.

$$
H^{n}\left(A^{*}\right) \rightarrow H^{n}\left(B^{*}\right) \rightarrow H^{n}\left(C^{*}\right) \stackrel{\partial}{\rightarrow} H^{n+1}\left(A^{*}\right) \rightarrow H^{n+1}\left(B^{*}\right) \rightarrow H^{n+1}\left(C^{*}\right)
$$

Joining these exact sequence for every $n$ forms the long exact sequence, as required.

\subsubsection{Bi-complexes}

\section{Definition 3.2.10. Bi-complex}

A bi-complex is a collection of graded vector spaces $\left\{C^{p, q}\right\}_{p, q \in \mathbb{Z}}$ and maps $d^{p}: C^{p, *} \rightarrow$ $C^{p+1, *}$ and $d^{q}: C^{*, q} \rightarrow C^{*, q+1}$ such that $d^{p+1} \circ d^{p}=d^{q+1} \circ d^{q}=d^{q+1} \circ d^{p}+d^{p+1} \circ d^{q}=0$. It can be visualised as the lattice below (note that this diagram is not required to commute). 


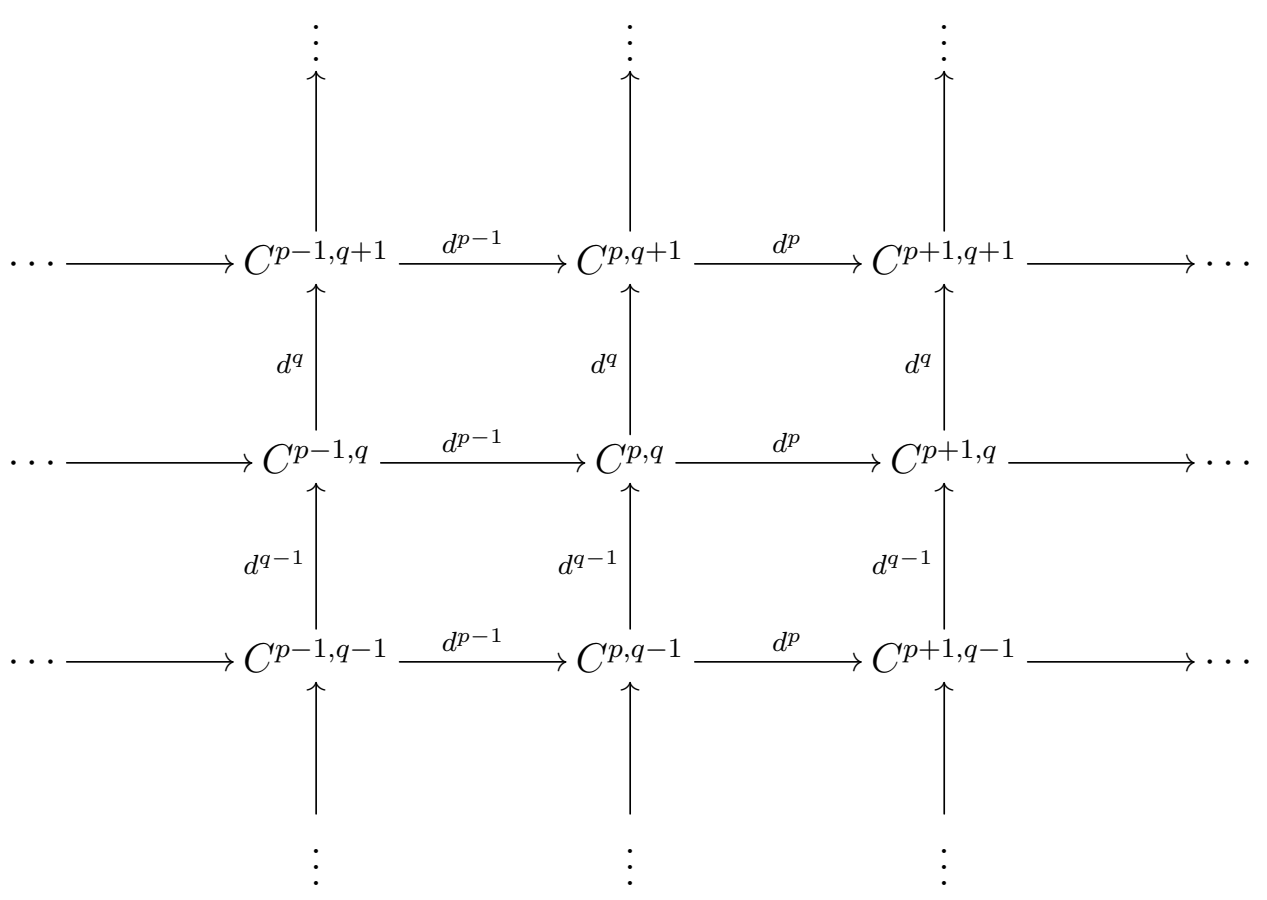

Note that $\left\{d^{p}\right\}_{p \in \mathbb{Z}}$ and $\left\{d^{q}\right\}_{q \in \mathbb{Z}}$ are not necessarily chain maps, as they don't commute with differentials.

\subsubsection{Mapping cones}

It will be useful when talking about the Khovanov homology to have some more constructions from homological algebra. The next construction we introduce is the mapping cone of a chain map $f^{*}$, denoted Cone $f^{*}$.

Consider a chain map $f^{*}: B^{*} \rightarrow C^{*}$. The mapping cone, denoted Cone $\left(f^{*}\right)$ is the chain complex $B^{*} \oplus C^{*}[-1]$ where the $n$th differential $d$ is defined as:

$$
d(b, c):=\left(d_{B} b, f b-d_{C} c\right) \quad \text { for every } b \in B^{n}, c \in C^{n-1}
$$

where $d_{B}, d_{C}$ are the differentials of $B^{*}$ an $C^{*}$ respectively, and the negatives denote additive inverses. 
Since the elements of Cone $\left(f^{*}\right)$ can be written as ordered pairs, and the differential is $R$-linear, the differentials can be written as matrices as follows, and we will do so accordingly. Also, composition of these $R$-linear maps therefore can be represented by matrix multiplication.

$$
\left(\begin{array}{cc}
d_{B}^{n+1} & 0 \\
f^{n+1} & -d_{C}^{n}
\end{array}\right)
$$

Recall that $d_{C}^{n} f^{n}=f^{n+1} d_{B}^{n}$ for every $n$, since $f^{*}$ is a chain map. Therefore, the mapping cone is indeed a cochain complex since:

$$
\left(\begin{array}{cc}
d_{B}^{n+1} & 0 \\
f^{n+1} & -d_{C}^{n}
\end{array}\right)\left(\begin{array}{cc}
d_{B}^{n} & 0 \\
f^{n} & -d_{C}^{n-1}
\end{array}\right)=\left(\begin{array}{cc}
d_{B}^{n+1} d_{B}^{n} & 0 \\
f^{n+1} d_{B}^{n}-d_{C}^{n} f^{n} & d_{C}^{n} d_{C}^{n-1}
\end{array}\right)=\left(\begin{array}{ll}
0 & 0 \\
0 & 0
\end{array}\right)
$$

as required.

\subsubsection{Chain homotopies}

Chain homotopies form an equivalence relation on chain maps between chain complexes. Much like homotopy-equivalent spaces having the same homology, chain homotopy equivalent complexes have the same homology.

\section{Chain homotopies}

Consider a cochain complex map $f^{*}: A^{*} \rightarrow B^{*}$ where the chain complexes $A^{*}$ and $B^{*}$ have differentials $d_{A}^{*}$ and $d_{B}^{*}$ respectively. $f^{*}$ is null-homotopic if there exists a collection of maps $\left\{s^{n}: A^{n} \rightarrow B^{n-1}\right\}$ such that $f^{n}=d_{B}^{n-1} s^{n}+s^{n+1} d_{A}^{n}$ for every $n$.

For clarity, we can consider the following non-commuting diagram. 


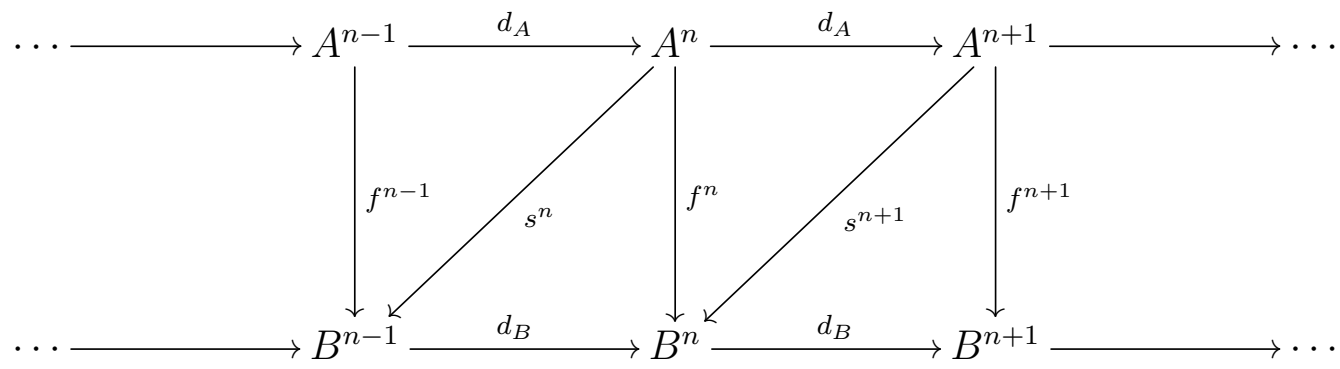

Two chain complex maps $f^{*}, g^{*}: A^{*} \rightarrow B^{*}$ are called chain homotopic if their difference is null-homotopic. Chain homotopic maps form an equivalence relation on all chain maps from $A^{*} \rightarrow B^{*}$, written $\simeq$. Therefore, since $f^{*}$ and $g^{*}$ are homotopic if and only if $f^{*}-g^{*}$ is null-homotopic, so $f^{*} \simeq g^{*}$ if and only if $(f-g)^{*}=f^{*}-g^{*} \simeq 0^{*}$.

Two chain complexes $A^{*}$ and $B^{*}$ are called chain homotopy equivalent, denoted $A^{*} \sim B^{*}$ if there exists chain complex maps $f^{*}: A^{*} \rightarrow B^{*}$ and $g^{*}: B^{*} \rightarrow A^{*}$ such that $f^{*} g^{*} \simeq \mathrm{id}_{B^{*}}$ and $g^{*} f^{*} \simeq \operatorname{id}_{A^{*}}$. This forms an equivalence relation on chain complexes.

As previously discussed, chain maps induce maps on the homology modules of the chain complex. That is $f^{*}: A^{*} \rightarrow B^{*}$ induces a map $\bar{f}^{n}: H^{n}\left(A^{*}\right) \rightarrow H^{n}\left(B^{*}\right)$ for every $n$.

Possibly the most useful property of chain homotopy is that chain homotopic maps induce the same homomorphism on homology modules. This theorem implies that it is sufficient to construct a chain homotopy equivalence between two chain complexes to show they are quasi-isomorphic. This is shown explicitly below.

Proposition 3.2.11. Let $f^{*}, g^{*}$ be chain homotopic chain maps, so $f^{*} \simeq g^{*}$. Then the induced maps of $f^{*}$ and $g^{*}$ on homology are equal: $\overline{f^{n}}=\overline{g^{n}}: H^{n}\left(A^{*}\right) \rightarrow H^{n}\left(B^{*}\right)$.

Proof. Suppose $f^{*}, g^{*}: A^{*} \rightarrow B^{*}$ are chain homotopic chain maps. Then there exists a family of maps $\left\{s^{n}: A^{n} \rightarrow B^{n-1}\right\}_{n \in \mathbb{Z}}$ such that $f^{n}-g^{n}=d_{B}^{n-1} s^{n}+s^{n+1} d_{A}^{n}$ for each 
$n$. Take some $a \in \operatorname{ker} d_{A}^{n}$. Then we can rewrite $f^{n}(a)-g^{n}(a)$ as follows.

$$
\begin{aligned}
f^{n}(a)-g^{n}(a) & =\left(d_{B}^{n-1} s^{n}+s^{n+1} d_{A}^{n}\right)(a) \\
& =d_{B}^{n-1} s^{n}(a)+s^{n+1} d_{A}^{n}(a) \\
& =d_{B}^{n-1} s^{n}(a)
\end{aligned}
$$

This shows $f(a)-g(a) \in \operatorname{im} d_{B}$. Therefore, $\overline{f^{n}}([a])-\overline{g^{n}}([a])=[f(a)-g(a)]=[0]$ for every $a \in \operatorname{ker} d_{A}^{n}$, so $\overline{f^{n}}=\overline{g^{n}}$, as required.

Using Proposition 3.2.11, it is straightforward to see that chain homotopy equivalent complexes have isomorphic homology groups. This can be seen as follows. Suppose $A^{*} \sim B^{*}$ are chain homotopy equivalent via chain maps $f^{*}: A^{*} \rightarrow B^{*}$ and $g^{*}: B^{*} \rightarrow$ $A^{*}$. Then $\overline{\left(f^{n} g^{n}\right)}=\overline{f^{n}} \overline{g^{n}}=\overline{\operatorname{id}_{B^{*}}}=\mathrm{id}_{H^{n}(B)}$, and similarly $\overline{g^{n}} \overline{f^{n}}=\operatorname{id}_{H^{n}(A)}$, which implies that $\overline{g^{n}}$ and $\overline{f^{n}}$ are both isomorphisms for each $n$, and so $H^{n}\left(A^{*}\right) \cong H^{n}\left(B^{*}\right)$ for every $n$.

\section{$3.3 \quad \mathbb{Z}$-graded $R$-modules}

The algebraic structures we will be using for most of this thesis are graded vector spaces - vector spaces with some additional structure. In this section, we define them and translate some of the constructions in the previous section from $R$-modules to $\mathbb{Z}$-graded $R$-modules, and $R$-homomorphism to $\mathbb{Z}$-graded $R$-homomorphisms.

We use graded vector spaces as opposed to vector spaces since one can 'encode' a polynomial in these structures by considering the graded dimension of graded vector spaces - an analogue of dimension of vector spaces.

\section{Definition 3.3.1. Graded vector spaces}

An $A$-graded vector space $W$ over the field $K$ is the direct sum $\bigoplus_{\alpha \in A} W_{\alpha}$, where each $W_{\alpha}$ is a vector space over $K$. The elements of $W_{\alpha}$ in $W$ are said to have degree $\alpha$, or to have $q$-grading $\alpha$. A homogeneous element of degree $\alpha$ is an element in $W_{\alpha}$. 
Note that the set $A$ can have some additional structure (for example, $A$ could be a group).

For convenience, the graded vector space will be written as the direct sum of the vector spaces in each dimension which are non-zero, with subscripts referring to its dimension. For example, for $\alpha_{i} \in A$, where $i \neq j$ implies $\alpha_{i} \neq \alpha_{j}$, and $n_{i} \in \mathbb{N}$, $K_{\alpha_{1}}^{n_{1}} \oplus K_{\alpha_{2}}^{n_{2}} \oplus \cdots \oplus K_{\alpha_{k}}^{n_{k}}$ is the $A$-graded $K$-vector space which is $K^{n_{i}}$ in degree $\alpha_{i}$.

Definition 3.3.2. Maps between graded vector spaces A map between $A$ graded vector spaces $L: W \rightarrow W^{\prime}$ is called a graded linear map if it preserves the grading, i.e. $L\left(W_{\alpha}\right) \subseteq W_{\alpha^{\prime}}$. In other words, the map $L$ is a collection of linear maps $\left\{L_{\alpha}: W_{\alpha} \rightarrow W_{\alpha}^{\prime}\right\}_{\alpha \in A}$ between associated vector spaces of degree $\alpha$.

Let $(A,+)$ be a set with a commutative binary operation $+: A \times A \rightarrow A$. $\mathrm{A}$ homogeneous linear map of degree $\beta$ is a map $L: W \rightarrow W^{\prime}$ such that $L\left(W_{\alpha}\right) \subseteq W_{\alpha+\beta}$. This means if 0 is the identity element of $(A,+)$, a homogeneous linear map of degree 0 is a graded linear map.

Example 3.3.3. One can think of the set of polynomials with real coefficients, $\mathbb{R}[X]$ as an $\mathbb{N}$-graded vector space. In this case, the coefficient of $X^{n}$ corresponds to the same element in degree $n$ of the graded vector space. So in degree $n \in \mathbb{N}$ of the graded vector space is a 1 -dimensional vector space over $\mathbb{R}$. The linear map that brings a polynomial $p(X)$ to $X p(X)$ is a graded linear map of degree 1 .

\section{Definition 3.3.4. Direct sum of graded vector spaces}

Consider $A$-graded vector spaces $W=\bigoplus_{\alpha \in A} W_{\alpha}$ and $W^{\prime}=\bigoplus_{\alpha \in A} W_{\alpha}^{\prime}$. The direct sum of $W$ and $W^{\prime}$ is the $A$-graded vector space is defined as follows.

$$
\left(W \oplus W^{\prime}\right)_{\alpha}:=\left(W_{\alpha} \oplus W_{\alpha}^{\prime}\right)
$$

We now will describe the tensor product of graded vector spaces. Recall in Example 3.1.1 that we showed that for every $R$-module $W, R \otimes W \cong W \cong W \otimes R$. So, the 
tensor product with $R$ will not change the $R$-module. The anologue of this object for $\mathbb{Z}$-graded $R$ modules is the following $R$-module, denoted $R_{0}$.

$$
\left(R_{0}\right)_{i}:= \begin{cases}R & : i=0 \\ 0 & : \text { otherwise }\end{cases}
$$

\section{Definition 3.3.5. Tensor product of graded vector spaces}

Recall a semi-group is a set with a commutative associative binary operation. Consider $A$-graded vector spaces $W$ and $W^{\prime}$ where $(A, *)$ is a semi-group. Let $i, j, k$ be elements of $A$. The tensor product of $W$ and $W^{\prime}$ is the $A$-graded vector space is defined for all $A$-gradings $k$ by:

$$
\left(W \otimes W^{\prime}\right)_{k}:=\bigoplus_{i * j=k}\left(W_{i} \otimes W_{j}^{\prime}\right)
$$

For $m \in \mathbb{N}$, the $m$ th tensor power of a graded vector space $W$ is written $W^{\otimes m}$.

$$
W^{\otimes m}:=\underbrace{W \otimes W \otimes \cdots \otimes W}_{m}
$$

Example 3.3.6. Recall $\mathbb{Z} / 2 \mathbb{Z}$ is the group of order 2 . Let $W$ be the $\mathbb{Z} / 2 \mathbb{Z}$-graded vector space which is 1 -dimensional over $\mathbb{Q}$ in degrees 0 and 1 , i.e. $W_{0}=W_{1}=\mathbb{Q}$. For convenience, this is written $\mathbb{Q}_{0} \oplus \mathbb{Q}_{1}$.

Then the tensor product $W \otimes W$ is the graded vector space:

$$
\begin{aligned}
& (W \otimes W)_{0}=\left(\mathbb{Q}_{0} \otimes \mathbb{Q}_{0}\right) \oplus\left(\mathbb{Q}_{1} \otimes \mathbb{Q}_{1}\right) \cong \mathbb{Q}^{2} \\
& (W \otimes W)_{1}=\left(\mathbb{Q}_{0} \otimes \mathbb{Q}_{1}\right) \oplus\left(\mathbb{Q}_{1} \otimes \mathbb{Q}_{0}\right) \cong \mathbb{Q}^{2}
\end{aligned}
$$

Here we are using that $\mathbb{Q} \otimes \mathbb{Q}=\mathbb{Q}$, where $\mathbb{Q}$ represents one dimensional vector spaces over $\mathbb{Q}$. Note that the graded dimension of $V$ is independent of field of the vector space.

From now on, we will consider only $\mathbb{Z}$-graded vector spaces. 


\section{Definition 3.3.7. Graded dimension of graded vector spaces}

The graded dimension, denoted $q$ dim, of a $\mathbb{Z}$-graded vector space $W=\bigoplus_{k \in \mathbb{Z}} W_{k}$ is a polynomial in variable $q$, defined as follows.

$$
q \operatorname{dim}\left(\bigoplus_{k \in \mathbb{Z}} W_{k}\right):=\sum_{k} q^{k} \operatorname{dim}\left(W_{k}\right)
$$

Example 3.3.8. From the point of view of the Khovanov homology, a very appropriate graded vector space to consider is the $\mathbb{Z}$-graded vector space $V$ over the field $K$ generated by one element $v_{+}$in degree 1 and one element $v_{-}$in degree -1 . The specific $\mathbb{Z}$-gradings of the graded vector space are:

$$
V_{i}:= \begin{cases}\operatorname{span}_{K}\left\{v_{+}\right\} & : i=+1 \\ \operatorname{span}_{K}\left\{v_{-}\right\} & : i=-1 \\ 0 & : \text { otherwise }\end{cases}
$$

In other words,

$$
V=K_{-1} \oplus K_{+1}
$$

The only $q$-gradings with non-trivial dimensions are \pm 1 , so we only consider a sum of $q^{1}$ and $q^{-1}$. Since the dimensions of the spaces at these $q$-gradings are exactly one, the graded dimension of this graded vector space is $q+q^{-1}$. Also, observe that $q+q^{-1}$ is the unnormalised Jones Polynomial of the unknot! This graded vector space will resurface in the next chapter.

In this latest example, we see a construction of a graded vector space that is related to a polynomial by its graded dimension. In fact, it 'encodes' this polynomial in its structure. The next definitions and propositions show some other manipulations of graded vector spaces which will have the necessary effect on their graded dimensions.

Example 3.3.9. We give another example of the tensor product, but this time with the $\mathbb{Z}$-graded $K$-vector space defined in Example 3.3.8. So, $V \cong K_{-1} \oplus K_{+1}$. Then, 
we have the following.

$$
\begin{aligned}
V \otimes V & =\left(K_{-1} \oplus K_{+1}\right) \otimes\left(K_{-1} \oplus K_{+1}\right) \\
& =\left(K_{-1} \otimes K_{-1}\right) \oplus\left(K_{1} \otimes K_{-1}\right) \oplus\left(K_{-1} \otimes K_{+1}\right) \oplus\left(K_{+1} \otimes K_{+1}\right) \\
& =(K \otimes K)_{-2} \oplus(K \otimes K)_{0} \oplus(K \otimes K)_{0} \oplus(K \otimes K)_{+2} \\
& \cong(K)_{-2} \oplus(K)_{0} \oplus(K)_{0} \oplus(K)_{+2} \\
& =(K)_{-2} \oplus\left(K^{2}\right)_{0} \oplus(K)_{+2}
\end{aligned}
$$

More generally, we can find $V^{\otimes k}$ for any $k \in \mathbb{N}$. The minimum non-trivial $q$-grading is $-k$, which is generated by

$$
\underbrace{K_{-1} \otimes K_{-1} \otimes \cdots \otimes K_{-1}}_{k} \cong K
$$

The next non-trivial $q$-grading is generated by all permutations of the following tensor product,

$$
\underbrace{K_{-1} \otimes K_{-1} \otimes \cdots \otimes K_{-1} \otimes K_{+1}}_{k} \cong K
$$

of which there are $k$. Continuing the pattern, we see there are $k$ choose $i,\left(\begin{array}{l}k \\ i\end{array}\right)$, different ways to arrange $i$ of $K_{+1}$ and $k-i$ of the $K_{-1}$, which will generate the space in $q$-grading $i-(k-i)=-k+2 i$. Hence, we can represent $V^{\otimes k}$ by the following direct sum over the $q$-gradings.

$$
V^{\otimes k}=\bigoplus_{0 \leq i \leq k} K_{-k+2 i}^{\left(\begin{array}{l}
k \\
i
\end{array}\right)}
$$

\section{Definition 3.3.10. Shifts in grading}

Take some $l \in \mathbb{Z}$. For a $\mathbb{Z}$-graded vector space $W=\bigoplus_{k \in \mathbb{Z}} W_{k}$, the degree shift operator, denoted $\cdot\{l\}$ defines a new graded vector space as follows.

$$
W\{l\}_{k}:=W_{k-l}
$$

Hence, if $l$ is positive, the operator $\cdot\{l\}$ "shifts" the graded vector space "up", or in 
the positive direction, by $l$ places. If $l$ is negative, the operator $\cdot\{l\}$ shifts the graded vector space in the negative direction.

One can think of this shift as a shift isomorphism, $\cdot\{l\}: W \cong W\{l\}$ which is a homogenous map of degree $l$ (and hence isn't strictly an isomorphism by our definition), where each map $W_{k} \stackrel{\equiv}{\rightarrow} W_{k-l}$ is an automorphism. Therefore, the shift sends an element $x \in W_{k}$ to $x \in W\{l\}_{k}$, so, if the degree of $x$ in $W$ is $k$, then the degree of $x$ in $W\{l\}$ is $k-l$.

\section{Proposition 3.3.11. Graded dimensions under the direct sum, the tensor} product, and grade shifts

Consider $\mathbb{Z}$-graded vector spaces $W$ and $W^{\prime}$.

1. $q \operatorname{dim}\left(W \oplus W^{\prime}\right)=q \operatorname{dim}(W)+q \operatorname{dim}\left(W^{\prime}\right)$

2. $q \operatorname{dim}\left(W \otimes W^{\prime}\right)=q \operatorname{dim}(W) \cdot q \operatorname{dim}\left(W^{\prime}\right)$

3. $q \operatorname{dim}(W\{l\})=q^{l} \cdot q \operatorname{dim}(W)$

Proof. 1. The $q$-dimension of a direct sum is the sum of the $q$-dimensions of the two graded vector spaces. This follows directly from that two vector spaces $U$ and $U^{\prime}, \operatorname{dim}\left(U \oplus U^{\prime}\right)=\operatorname{dim} U+\operatorname{dim} U^{\prime}$. So, at each grading, $\operatorname{dim}\left(W_{k} \oplus W_{k}^{\prime}\right)=$ $\operatorname{dim} W_{k}+\operatorname{dim} W_{k}^{\prime}$. Hence we have the following equations.

$$
\begin{aligned}
q \operatorname{dim}\left(W \oplus W^{\prime}\right) & =\sum_{k \in \mathbb{Z}} q^{k} \operatorname{dim}\left(\left(W \oplus W^{\prime}\right)_{k}\right) \\
& =\sum_{k \in \mathbb{Z}} q^{k} \operatorname{dim}\left(W_{k} \oplus W_{k}^{\prime}\right) \\
& =\sum_{k \in \mathbb{Z}} q^{k}\left(\operatorname{dim} W_{k}+\operatorname{dim} W_{k}^{\prime}\right) \\
& =\sum_{k \in \mathbb{Z}} q^{k} \operatorname{dim} W_{k}+\sum_{k \in \mathbb{Z}} q^{k} \operatorname{dim} W_{k}^{\prime} \\
& =q \operatorname{dim}(W)+q \operatorname{dim}\left(W^{\prime}\right)
\end{aligned}
$$


2. The $q$-dimension of a tensor product is the multiple of the $q$-dimensions of the two graded vector spaces. Similarly, this follows from the relation $\operatorname{dim}\left(U \otimes U^{\prime}\right)=$ $\operatorname{dim} U \cdot \operatorname{dim} U^{\prime}$

$$
\begin{aligned}
q \operatorname{dim}\left(W \otimes W^{\prime}\right) & =\sum_{k \in \mathbb{Z}} q^{k} \operatorname{dim}\left(\left(W \otimes W^{\prime}\right)_{k}\right) \\
& =\sum_{k \in \mathbb{Z}} q^{k} \operatorname{dim}\left(\bigoplus_{\substack{j+j^{\prime}=k \\
k \in \mathbb{Z}}} W_{j} \otimes W_{j^{\prime}}^{\prime}\right) \\
& =\sum_{k \in \mathbb{Z}} q^{k}\left(\sum_{\substack{j+j^{\prime}=k \\
k \in \mathbb{Z}}} \operatorname{dim}\left(W_{j} \otimes W_{j^{\prime}}^{\prime}\right)\right) \\
& =\sum_{k \in \mathbb{Z}} q^{k}\left(\sum_{\substack{j+j^{\prime}=k \\
k \in \mathbb{Z}}} \operatorname{dim} W_{j} \cdot \operatorname{dim} W_{j^{\prime}}^{\prime}\right) \\
& =\sum_{k \in \mathbb{Z}}\left(\sum_{\substack{j+j^{\prime}=k \\
k \in \mathbb{Z}}} q^{j+j^{\prime}} \operatorname{dim} W_{j} \cdot \operatorname{dim} W_{j^{\prime}}^{\prime}\right) \\
& =\left(\sum_{j \in \mathbb{Z}} q^{j} \operatorname{dim} W_{j}\right) \cdot\left(\sum_{j^{\prime} \in \mathbb{Z}} q^{j^{\prime}} \operatorname{dim} W_{j^{\prime}}^{\prime}\right) \\
& =q \operatorname{dim}(W) \cdot q \operatorname{dim}\left(W^{\prime}\right)
\end{aligned}
$$

3. Lastly, the shift map $\cdot\{l\}$ alters the $q$-dimension by $q^{l}$.

$$
\begin{aligned}
q \operatorname{dim}(W\{l\}) & =\sum_{k \in \mathbb{Z}} q^{k} \operatorname{dim}\left(W\{l\}_{k}\right) \\
& =\sum_{k \in \mathbb{Z}} q^{k} \operatorname{dim}\left(W_{k-l}\right) \\
& =q^{l} \sum_{k \in \mathbb{Z}} q^{k-l} \operatorname{dim}\left(W_{k-l}\right) \\
& =q^{l} \sum_{k-l \in \mathbb{Z}} q^{k-l} \operatorname{dim}\left(W_{k-l}\right) \\
& =q^{l} \cdot q \operatorname{dim}(W)
\end{aligned}
$$


Thinking back to the relations used to compute the Kauffman Bracket, these relations provide one with a way to construct a graded vector space with a specific graded dimension. For example, in relation two of Definition 2.2.1, $\langle\bigcirc \amalg D\rangle=\left(q+q^{-1}\right)\langle D\rangle$. To find a graded vector space with graded dimension that satisfies this relation, we see from (2) in Proposition 3.3.11 that $q \operatorname{dim}\left(W \otimes W^{\prime}\right)=q \operatorname{dim}(W) \cdot q \operatorname{dim}\left(W^{\prime}\right)$. So if $q \operatorname{dim}(V)=q+q^{-1}$, we know that the right hand side of the equation can be represented by a tensor product, $\otimes$, of graded vector spaces.

This is not exactly what Khovanov has done. Instead of finding a graded vector space for every knot diagram which is related to its Kauffman bracket by its graded dimension, he found a chain complex of graded vector spaces for every knot diagram $D$. This will be defined in the next chapter.

\section{Homology on graded vector spaces}

The definitions of chain complexes and homology of $R$-modules are defined similarly for graded vector spaces. We will make a few brief recollections about homology in the context of graded vector spaces.

A cochain complex of graded vector spaces over the field $K$ is a sequence of graded vector spaces $W^{*}:=\left\{W^{i}\right\}_{i \in \mathbb{Z}}$ and graded $K$-linear maps $\left\{d^{i}: W^{i} \rightarrow W^{i+1}\right\}_{i \in \mathbb{Z}}$ such that the composition $d^{i+1} \circ d^{i}=0$.

$$
\cdots \rightarrow W^{i-1} \stackrel{d^{i-1}}{\longrightarrow} W^{i} \stackrel{d^{i}}{\rightarrow} W^{i+1} \rightarrow \cdots
$$

In this context, the differentials of the chain complex are to be graded linear maps, so they must be of degree zero. Hence the $i$ th differential can be thought of as a collection of maps $d_{j}^{i}: W_{j}^{i} \rightarrow W_{j}^{i+1}$ for every $j \in \mathbb{Z}$. The height shift operator $\cdot[s]$ for $s \in \mathbb{Z}$ is also defined on cochain complexes of graded vector spaces.

$$
W^{r}[s]:=W^{r-s}
$$

We now cover some facts that will be useful for showing the Jones polynomial is the 
graded Euler characteristic of the Khovanov bracket.

\section{Definition 3.3.12. Graded Euler characteristic}

The graded Euler characterisitic, $\chi_{q}$, of a bounded chain complex of graded vector spaces $W^{*}$ is the alternating sum of the graded dimensions of the homology graded vector spaces.

$$
\chi_{q}\left(W^{*}\right):=\sum_{i}(-1)^{i} q \operatorname{dim} H^{i}\left(W^{*}\right)
$$

Proposition 3.3.13. The graded Euler characteristic of a bounded chain complex of finitely generated graded vector spaces $C^{*}$ (with degree zero differentials) is equal to the alternating sums of the graded dimensions of the chain complex $C^{*}$.

Proof. The proof of the proposition follows from two facts. Firstly, for a linear map $f: A \rightarrow B, A \cong \operatorname{ker} f \oplus \operatorname{im} f$, and therefore $\operatorname{dim} A=\operatorname{dim}(\operatorname{ker} f)+\operatorname{dim}(\operatorname{im} f)$. Secondly, if $A$ is the quotient $B / C$, then $\operatorname{dim} A=\operatorname{dim} B-\operatorname{dim} C$.

Consider a bounded chain complex of finitely generated vector spaces, $C^{*}$, and without loss of generality we assume it is trivial in homological degree $<0$. There is an $r \in \mathbb{Z}$ such that $C^{i}=0$ for $i<0$ and $i>r$. We know from the above statements that $\operatorname{dim}\left(C^{i}\right)=\operatorname{dim}\left(\operatorname{ker} d^{i}\right)+\operatorname{dim}\left(\operatorname{im} d^{i}\right)$ and $\operatorname{dim}\left(H^{i}\left(C^{*}\right)\right)=\operatorname{dim}\left(\operatorname{ker} d^{i}\right)-\operatorname{dim}\left(\operatorname{im} d^{i-1}\right)$ which are all finite, hence we substitute this into the Euler characteristic of $C^{*}$.

$$
\begin{aligned}
\chi\left(C^{*}\right) & =\sum_{i}(-1)^{i} \operatorname{dim} H^{i}\left(C^{*}\right) \\
& =\sum_{i}(-1)^{i} \operatorname{dim}\left(\operatorname{ker} d^{i} / \operatorname{im} d^{i-1}\right) \\
& =\sum_{i}(-1)^{i}\left(\operatorname{dim}\left(\operatorname{ker} d^{i}\right)-\operatorname{dim}\left(\operatorname{im} d^{i-1}\right)\right) \\
& =\operatorname{dim}\left(\operatorname{ker} d^{0}\right)-\operatorname{dim}\left(\operatorname{im} d^{-1}\right)-\left(\operatorname{dim}\left(\operatorname{ker} d^{i}\right)-\operatorname{dim}\left(\operatorname{im} d^{i-1}\right)\right)+\cdots \\
& \quad \pm\left(\operatorname{dim}\left(\operatorname{ker} d^{r}\right)-\operatorname{dim}\left(\operatorname{im} d^{r-1}\right)\right) \\
& =\operatorname{dim}\left(C^{0}\right)-\operatorname{dim}\left(C^{1}\right)+\cdots \pm \operatorname{dim}\left(C^{r}\right) \\
& =\sum_{i}(-1)^{i} \operatorname{dim}\left(C^{i}\right)
\end{aligned}
$$


We have only shown that this is the case for vector spaces, so we now extend this to graded vector spaces. Thus, suppose $C^{*}$ is a cochain complex of graded vector spaces. Then the above equality is true for each $q$-grading of the graded vector space, $C_{j}^{*}$. The equalities below complete the proof.

$$
\begin{aligned}
\chi_{q}\left(C^{*}\right) & =\sum_{i}(-1)^{i} q \operatorname{dim} H^{i}\left(C^{*}\right) \\
& =\sum_{i}(-1)^{i}\left(\sum_{j} q^{j} \operatorname{dim}\left(H^{i}\left(C^{*}\right)_{j}\right)\right) \\
& =\sum_{j} q^{j}\left(\sum_{i}(-1)^{i} \operatorname{dim}\left(H^{i}\left(C^{*}\right)_{j}\right)\right) \\
& =\sum_{j} q^{j}\left(\sum_{i}(-1)^{i} \operatorname{dim}\left(C^{i}\right)_{j}\right) \\
& =\sum_{i}(-1)^{i}\left(\sum_{j} q^{j} \operatorname{dim}\left(C^{i}\right)_{j}\right) \\
& =\sum_{i}(-1)^{i} q \operatorname{dim}\left(C^{i}\right)
\end{aligned}
$$




\section{Chapter 4}

\section{The Khovanov homology}

In this chapter, we introduce the Khovanov homology, which is an invariant of knots. This is really a cohomology theory, but is called the Khovanov homology throughout the literature.

We begin this chapter with a description and then a definition of the Khovanov bracket. The Khovanov bracket satisfies three relations which are analogous to the three Kauffman bracket relations, but instead assigns a cochain complex of $\mathbb{Z}$-graded vector spaces to each knot diagram. However, the Khovanov bracket itself is not a link invariant. After assigning an orientation to a knot diagram, we assign the Khovanov bracket of the underlying knot diagram with a shift that depends on its orientation, called the Khovanov cochain complex. The Khovanov cohomology is the cohomology of this cochain complex of graded vector spaces, and is indeed a knot invariant.

For the majority of this chapter, the graded vector spaces we will look at are over a general field $K$. In particular, if we consider the Khovanov chain complex over the field $\mathbb{Q}$, we find that the Jones polynomial is the Euler characteristic of this chain complex. In fact, through little extra effort, everything we say here can be generalised to a commutative ring $R$ with identity, by replacing every instance of 'field' with 'commutative ring with identity'. Hence, the ' $\mathbb{Z}$-graded $K$-vector space' $V$ becomes the ' $\mathbb{Z}$-graded $R$-module' generated by an element $v_{+}$in degree 1 and one 
element $v_{-}$in degree -1 , but one can forget about this for almost the entire chapter.

\section{1 $\quad$ Setting the scene}

Here are some definitions and constructions that we will need to describe the Khovanov bracket. The notation in this section when referring to smoothings is the same as the notation of Chapter 2, but many of the points and definitions will be reiterated here.

\section{The $\mathbb{Z}$-graded $K$-vector space $V$}

First we recall a $\mathbb{Z}$-graded $K$-vector space, $V$, first defined in Chapter 3 . This is the graded vector space referred to as $V$ for the rest of this thesis. Let $V$ be generated by one element $v_{+}$in degree 1 and one element $v_{-}$in degree -1 . Specifically, the graded vector space is as follows.

$$
V_{i}:= \begin{cases}\operatorname{span}_{K}\left\{v_{+}\right\} & : i=+1 \\ \operatorname{span}_{K}\left\{v_{-}\right\} & : i=-1 \\ 0 & : \text { otherwise }\end{cases}
$$

In other words,

$$
V=K_{-1} \oplus K_{+1}
$$

Also, recall that the graded dimension of this $\mathbb{Z}$-graded vector space $V$, denoted $q \operatorname{dim}(V)$, is $q+q^{-1}$.

\section{The Flatten operation}

We now describe a 'Flatten' operation which takes a chain complex of chain complexes to a chain complex. First, it assigns a bi-complex to a chain complex of chain complexes, and then forms a chain complex from a bi-complex by taking each space to be a direct sum of a diagonal. We first describe how to get the bi-complex, and 
then describe how the Flatten operation works.

Let $\left(C^{p}\right)^{*}$ be a chain complex for every $p \in \mathbb{Z}$. Consider a chain complex of chain complexes. That is, consider the following chain complex of chain complexes $\left\{\left(C^{p}\right)^{*}\right\}_{p \in \mathbb{Z}}$ and differentials (that are chain complex maps) $\left(f^{p}\right)^{*}$.

$$
\cdots \rightarrow\left(C^{p-1}\right)^{*} \stackrel{\left(f^{p-1}\right)^{*}}{\longrightarrow}\left(C^{p}\right)^{*} \stackrel{\left(f^{p}\right)^{*}}{\longrightarrow}\left(C^{p+1}\right)^{*} \rightarrow \cdots
$$

Let $d^{p, q}$ be the $q$ th differential of $\left(C^{p}\right)^{*}$, and $f^{p, q}$ is the $q$ th chain map component of $\left(f^{p}\right)^{*}$. Then since $\left(f^{p}\right)^{*}$ is a chain map, we have $f^{p, q+1} \circ d^{p, q}=d^{p+1, q} \circ f^{p, q}$ by definition, as seen in the following commutative diagram. Also, we have that $d^{p} d^{p}=f^{q} f^{q}=0$.

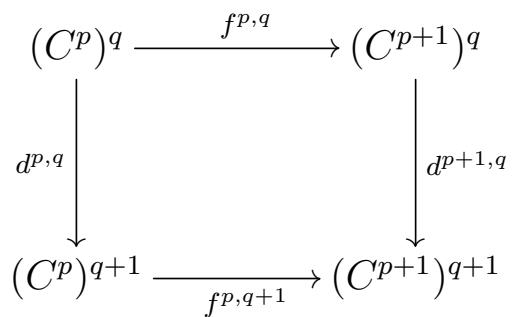

Furthermore, one can form a bi-complex, $\left\{C^{p, q}\right\}_{p, q \in \mathbb{Z}}$, from this chain complex of chain complexes by including minuses on the differentials to make each square anticommute. Specifically, the component $C^{p, q}$ is the object $\left(C^{p}\right)^{q}$ in the above notation. As for differentials, let the vertical maps $\left\{d_{v}:=d^{p, q}: C^{p, q} \rightarrow C^{p, q+1}\right\}_{p, q \in \mathbb{Z}}$ be the differentials of each $\left(C^{p}\right)^{*}$ in the sequence above, and let the horizontal maps $\left\{d_{h}: C^{p, q} \rightarrow C^{p+1, q}\right\}_{p, q \in \mathbb{Z}}$ be defined as the map $(-1)^{q}\left(f^{p}\right)^{q}$. 


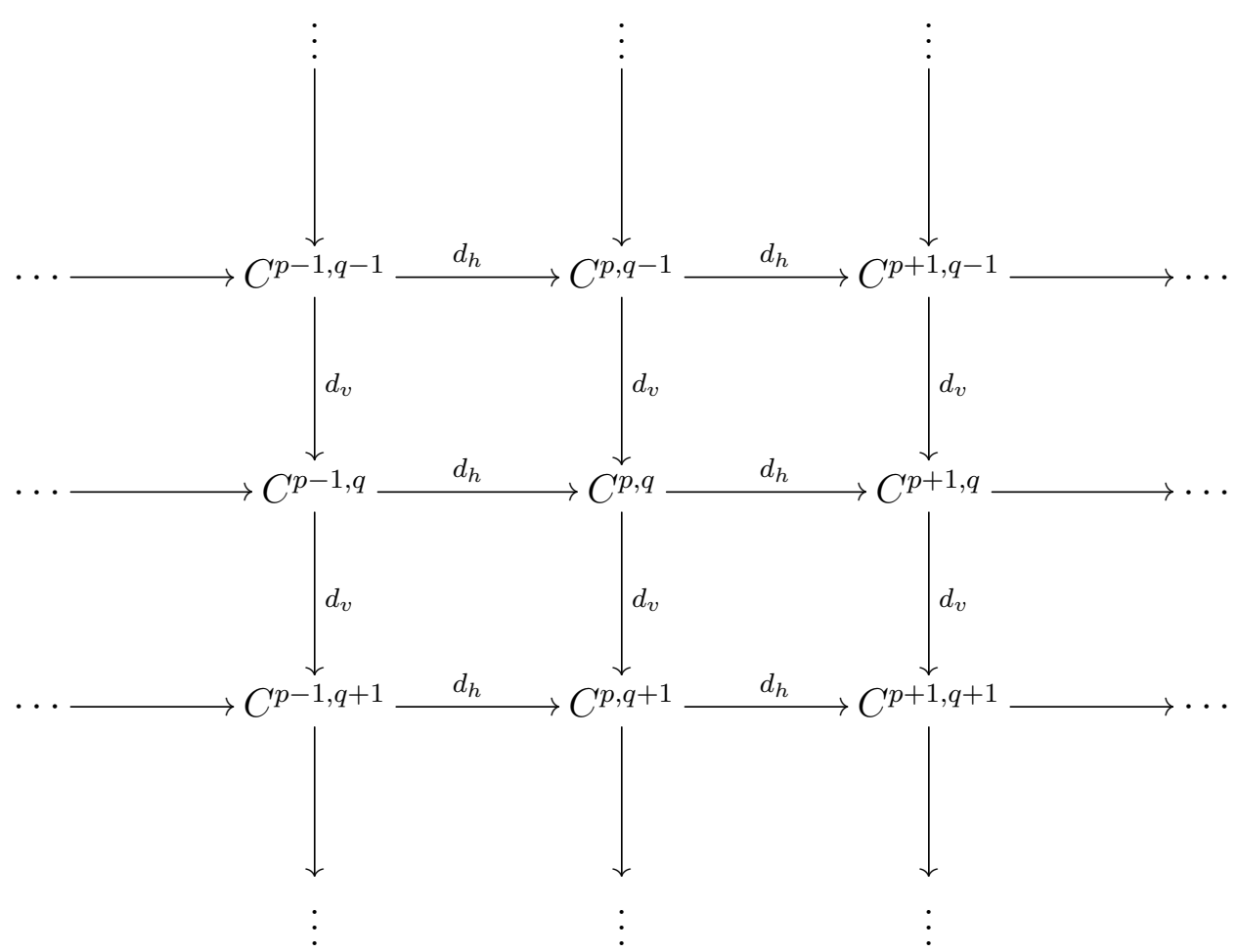

The sign change of the horizontal differentials in the previous paragraph implies that $d_{v}^{p+1, q} \circ d_{h}^{p, q}=d_{v}^{p+1, q} \circ(-1)^{q} f^{p, q}=(-1)^{q} f^{p, q+1} \circ d_{v}^{p, q}=(-1)^{q}(-1)^{-(q+1)} d_{h}^{p, q+1} \circ d_{v}^{p, q}=$ $-d_{h}^{p, q+1} \circ d_{v}^{p, q}$. Hence we have established anti-commutativity on each square of the diagram above. This means that now $\left\{d_{h}^{p, q}\right\}_{p, q \in \mathbb{Z}}$ and $\left\{d_{v}^{p, q}\right\}_{p, q \in \mathbb{Z}}$ are not necessarily chain maps. Note that we also know that $d_{v} d_{v}=d_{h} d_{h}=0$ as both $C^{p, *}$ and $C^{*, q}$ are chain complexes.

Now we have a bi-complex, we can define the flatten operation, which gives us another chain complex. The components of

$$
\text { Flatten }\left[\cdots \rightarrow\left(C^{p}\right)^{*} \stackrel{\left(f^{p}\right)^{*}}{\longrightarrow}\left(C^{p+1}\right)^{*} \rightarrow \cdots\right]
$$

are the direct sums of the diagonals of this bi-complex. Thus, the $n$th component of this new chain complex is

$$
\bigoplus_{p+q=n} C^{p, q}
$$


This collection of spaces along with the maps

$$
d^{n}=\sum_{p+q=n}\left(d_{v}^{p, q}+d_{h}^{p, q}\right)
$$

forms a chain complex. This is a chain complex from the anti-commutativity of the squares above and that $d_{v} d_{v}=d_{h} d_{h}=0$.

\section{Tensor product of a cochain complex and a module}

Consider a $\mathbb{Z}$-graded vector space $U$ and a chain complex of $\mathbb{Z}$-graded vector spaces $W^{*}$. We define the tensor product $U \otimes\left(W^{*}\right)$ to be the chain complex of graded vector spaces as follows. The graded vector space in homological degree $i$ is $\left\{U \otimes W^{i}\right\}$, and if $d^{i}$ is the $i$ th differential of $W^{*}$, then the $i$ th differential of $U \otimes\left(W^{*}\right) \operatorname{is} \operatorname{id}_{U} \otimes d^{i}$. This is a well defined cochain complex as $\left(\mathrm{id}_{U} \otimes d^{i+1}\right) \circ\left(\mathrm{id}_{U} \otimes d^{i}\right)=\left(\operatorname{id}_{U} \mathrm{id}_{U}\right) \otimes\left(d^{i+1} d^{i}\right)=$ $\operatorname{id}_{U} \otimes 0=0$.

\subsection{The Khovanov bracket}

We now construct the Khovanov bracket of a link diagram, which is a chain complex of graded vector spaces. Each of $\mathbf{K f} \mathbf{1}, \mathbf{K f \mathbf { 2 }}, \mathbf{K f} \mathbf{3}$ has a direct analogue to one of the following relations. We begin by describing the three relations we want the Khovanov bracket to satisfy, along with a description of what they imply. Next, we give a definition of the graded vector spaces in each degree of the chain complex, and then define the differentials of this chain complex. Finally, we will show that this definition does indeed satisfy the three Khovanov bracket relations. This list of relations is from [1].

The Khovanov bracket of a knot diagram $D$, written $\llbracket D \rrbracket$, is a cochain complex of $\mathbb{Z}$-graded $K$-vector spaces. It satisfies the following three relations.

$$
\begin{aligned}
& \text { Kh1 } \llbracket \emptyset \rrbracket=0 \rightarrow K \rightarrow 0 \\
& \text { Kh2 } \bigcirc \amalg D \rrbracket=V \otimes \llbracket D \rrbracket
\end{aligned}
$$


Kh3 $\llbracket \backslash \rrbracket=$ Flatten $\left[0^{*} \rightarrow \llbracket \bumpeq \rrbracket \rightarrow \llbracket\right)\left(\rrbracket\{1\} \rightarrow 0^{*}\right]$

We sometimes will refer to the chain complex $\llbracket \cdot \rrbracket$ with the superscript $\llbracket \cdot \rrbracket^{*}$. The graded vector space in homological degree $r$ will be denoted $\llbracket \cdot \rrbracket^{r}$.

Property Kh1 tells us the Khovanov bracket of the empty link is the chain complex which is only non-trivial in degree 0 , where the graded vector space is simply $K$ in $q$-degree 0 . Property $\mathbf{K h 2}$ defines the disjoint union of an unknot and any knot diagram $D$ in homological degree $r$ to be the tensor product of $V \otimes \llbracket D \rrbracket^{r}$, as explained in Section 4.1 .

The third rule, Kh3, relates the Khovanov bracket of a knot diagram $D$ to the Khovanov bracket of its smoothings. Explicitly, suppose $D$ is a knot diagram with $n>0$ crossings, and choose a crossing of $D$ which we refer to as the $i$ th crossing. Let $D_{0}$ be identical to $D$ but with a 0 -smoothing at the $i$ th crossing, and similarly $D_{1}$ be identical to $D$ but with a 1 -smoothing at the $i$ th crossing. Inside the brackets of the Flatten operation in $\mathbf{K h} \mathbf{3}$, there is the map of cochain complexes as follows (drawn horizontally rather than vertically for convenience). The top row lies in degree 0 , and the bottom row is in degree 1 . These are the only non-trivial rows in the diagram.

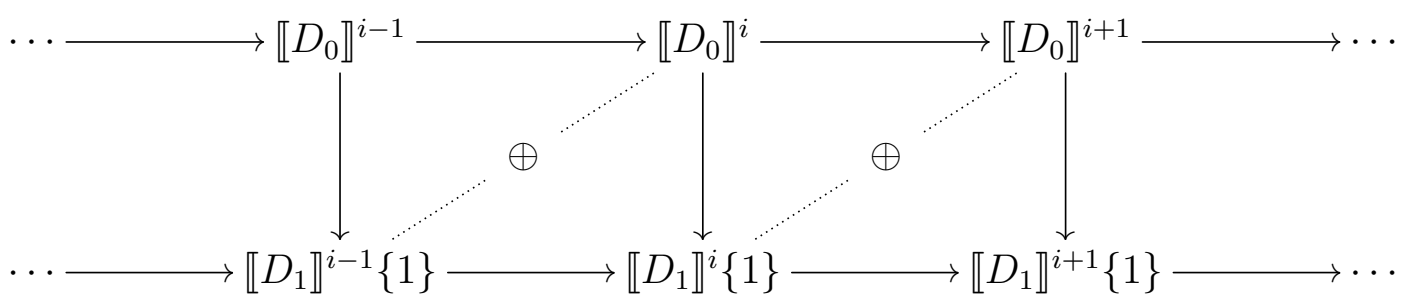

Hence, the $i$ th graded vector space in the cochain complex will be the graded vector space $\llbracket D_{0} \rrbracket^{i} \oplus \llbracket D_{1} \rrbracket^{i-1}\{1\}$, as shown by the dotted diagonal lines. We let the 0th graded vector space in the new "flattened" sequence be the diagonal that includes the graded vector space in the 0 th row and 0th column, which in this case is $\llbracket D_{0} \rrbracket^{0}$. Moreover, it also follows from all three rules that the chain complex $\llbracket \cdot \rrbracket$ will only be 
non-trivial in homological degrees $0 \leq i \leq n$ (recall $n$ is the number of crossings in $D)$. Therefore, we can rewrite the graded vector spaces in the cochain complex $\llbracket D \rrbracket$ as follows.

$$
\llbracket D \rrbracket^{i}= \begin{cases}\llbracket D_{0} \rrbracket^{i} & : i=0 \\ \llbracket D_{0} \rrbracket^{i} \oplus \llbracket D_{1} \rrbracket^{i-1}\{1\} & : 0<i<n \\ \llbracket D_{1} \rrbracket^{i-1}\{1\} & : i=n\end{cases}
$$

So far we have avoided discussing the differentials of the chain complex $\llbracket \cdot \rrbracket$, including the vertical arrows in the above diagram, but we will soon. The Flatten operation also defines "new" differentials from the current bi-complex.

Note also that $\mathrm{Kh} \mathbf{1}$ and $\mathbf{K h} \mathbf{2}$ specify the Khovanov bracket of a knot diagram of $k$ unlinked unknots in the plane.

$$
\llbracket k \text { unlinked unknots } \rrbracket=0 \rightarrow V^{\otimes k} \rightarrow 0
$$

Like the Kauffman bracket, the Khovanov bracket of a knot projection $D$ is also constructed from the total smoothings of $D$. Suppose $D$ is a knot projection with $n$ crossings that are ordered in some way. Recall that to every total smoothing of $D$, one can associate a string of length $n$ from the alphabet $\{0,1\}$, or an $\alpha \in\{0,1\}^{n}$. Also, each string can be associated to a vertex of the $n$-dimensional cube mentioned in Section 2.2. In other words, there is a bijective natural correspondence between the total smoothings of $D$ and elements $\alpha \in\{0,1\}^{n}$. This is "natural" in the sense that once there is an ordering of the crossings of the knot projection, then we can associate the smoothing of the $i$ th crossing to the $i$ th element in $\alpha$.

The total smoothing of a knot diagram $D$ is a link diagram with every crossing in $D$ replaced by a smoothing, and hence is a collection of $k$ unlinked unknots in the plane. To be explicit, the total smoothing of $D$ associated to $\alpha$ is as follows. If the 
$i$ th element in $\alpha$ is 0 , then the $i$ th crossing of $D$ is replaced with a 0 -smoothing, and similarly if $i$ th element in $\alpha$ is 1 , and is denoted $S_{\alpha}$. For a complete discussion, refer to Chapter 2 .

Let $V_{\alpha}$ be the graded vector space of the smoothing $S_{\alpha}$. Recall $k_{\alpha}$ is the number of cycles in $S_{\alpha}$, and $r_{\alpha}$ is the number of 1-smoothings of $S_{\alpha}$, also denoted $|\alpha|$. We define the graded vector space associated with the total smoothing $S_{\alpha}$ of $D$ to be the following.

$$
V_{\alpha}:=V^{\otimes k_{\alpha}}\left\{r_{\alpha}\right\}
$$

\section{Example 4.2.1. Khovanov bracket of the Hopf link}

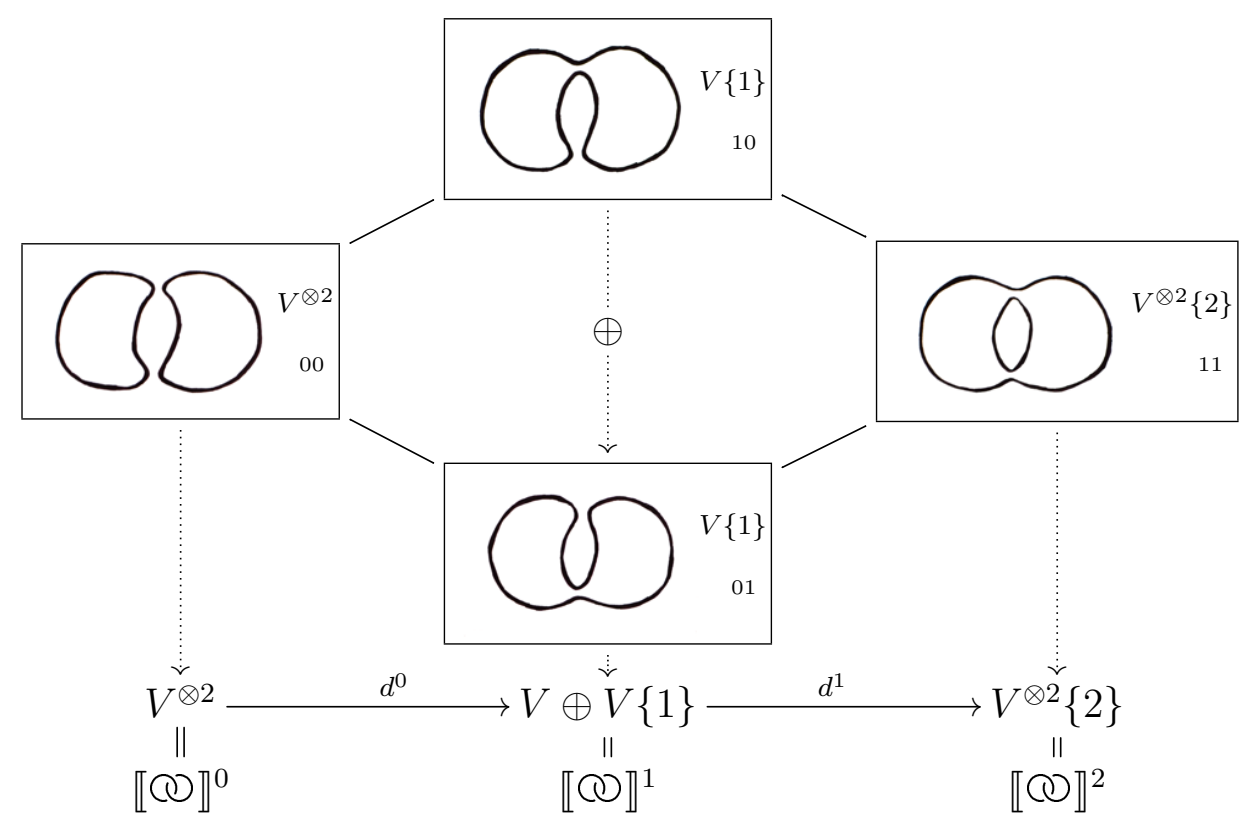

In the above diagram (which we note is similar to Example 2.2.3), the bottom line is the Khovanov bracket (though the differentials $d^{*}$ have not been defined). It sits with the leftmost graded vector space in chain complex degree 0 . Another way to think of the cochain complex of the Hopf link is seen in the table above. This is the 


\begin{tabular}{|c|c|c|c|c|}
\hline$m$ & -1 & 0 & 1 & 2 \\
\hline 4 & & & & $K$ \\
\hline 3 & & & & \\
\hline 2 & & $K$ & $K^{2}$ & $K^{2}$ \\
\hline 1 & & & & \\
\hline 0 & & $K^{2}$ & $K^{2}$ & $K$ \\
\hline-1 & & & & \\
\hline-2 & & $K$ & & \\
\hline
\end{tabular}

Khovanov bracket of the Hopf link where $V$ is a graded vector space over $K$. Here, $m$ is the $q$-grading and $r$ is the height or chain complex degree. In other words, each column represents a different graded vector space in the cochain complex of graded vector spaces. Only the non-zero entries are shown.

So, for example, at degree zero of the chain complex, the graded vector space is the direct sum of the column, $K_{-2} \oplus K_{0}^{2} \oplus K_{2}$. See Example 3.3 .9 for the explicit calculation of $V^{\otimes 2}$.

We now define the underlying collection of graded vector spaces of the Khovanov bracket. Once we define the differentials, and show that together this sequence does form a cochain complex, we will show that this Khovanov bracket satisfies the relations Kh1, Kh2 and Kh3.

\section{Definition 4.2.2. The spaces of the Khovanov bracket, $\llbracket \cdot \rrbracket$}

Let $D$ be a knot diagram with $n$ crossings which are ordered in some arbitrary way. Also, let $\alpha \in\{0,1\}^{n}$. The graded vector space in degree $r$ of the Khovanov bracket, $\llbracket D \rrbracket$, is defined to be the following direct sum.

$$
\llbracket D \rrbracket^{r}:=\bigoplus_{r_{\alpha}=r} V^{\otimes k_{\alpha}}\left\{r_{\alpha}\right\} \quad \text { for } 0 \leq r \leq n
$$

In degrees $r<0$ and $r>n$, the Khovanov bracket is trivial. 
We claim that this definition of the Khovanov bracket is independent of the ordering of the crossings of the knot diagram $D$. This amounts to considering a permutation, $\sigma:\{0,1\}^{n} \rightarrow\{0,1\}^{n}$ on $n$ elements, which rearranges the crossings. It follows that $|\alpha|=|\sigma(\alpha)|$, so the height is unchanged, though the smoothing $S_{\alpha}$ will not necessarily be the same as $S_{\sigma(\alpha)}$. Therefore, consider the string $\alpha^{\prime}:=\sigma(\alpha)$. Then the smoothing $S_{\alpha^{\prime}}=S_{\sigma(\alpha)}$, and so $k_{\alpha^{\prime}}=k_{\sigma(\alpha)}$. Therefore, a permutation will only change the ordering in the direct sum,

$$
\bigoplus_{r_{\alpha}=r} V^{\otimes k_{\alpha}}\left\{r_{\alpha}\right\}
$$

for each $r$, since $\{\alpha:|\alpha|=r\}=\{\sigma(\alpha):|\alpha|=r\}$. Note that we haven't actually defined a way to order the $V_{\alpha}$ in the direct sum above, so in some way we were defining the bracket up to "ways to order this direct sum".

\section{The edges of the $n$-dimensional cube of smoothings}

Let $\alpha, \alpha^{\prime} \in\{0,1\}^{n}$ such that $\alpha$ and $\alpha^{\prime}$ differ in exactly one place, which we refer to as the $i$ th crossing, and $|\alpha|<\left|\alpha^{\prime}\right|$ (in other words, this implies $|\alpha|+1=\left|\alpha^{\prime}\right|$ ). Let $\zeta \in\{0,1, \star\}^{n}$ such that $\zeta$ is the same as $\alpha$ and $\alpha^{\prime}$ everywhere except in the $i$ th crossing (where $\alpha$ and $\alpha^{\prime}$ differ) which is replaced with $\star$. We will refer to this crossing in $D$ as the $i$ th crossing. In an $n$-dimensional cube, there is exactly one edge between $\alpha$ and $\alpha^{\prime}$, which will be denoted $\zeta$ as it was just defined. All edges in the cube are of this form.

We define a graded linear map $d_{\zeta}: V_{\alpha} \rightarrow V_{\alpha^{\prime}}$ along each edge of the $n$-dimensional cube of (the graded vector spaces of) smoothings, and as in the previous description, the subscript $\zeta$ denotes the edge, and depends on $\alpha, \alpha^{\prime}$. As a slight abuse of notation, we will sometimes say cube of smoothings when we really mean the cube of the graded vector spaces associated to the smoothings.

Each $d_{\zeta}$ is a graded linear map between two smoothings which only affects the graded vector spaces of the cycles that "intersect" the $i$ th crossing. In other words, consider $\alpha, \alpha^{\prime}$ which differ only in the $i$ th place as before, and their total smoothings $S_{\alpha}, S_{\alpha^{\prime}}$. The two strands in the 0 -smoothing of the $i$ th crossing and the 1-smoothing of the 
$i$ th crossing will either be a part of one cycle or two cycles. In the case that the 0 -smoothing of the $i$ th crossing is part of a single cycle, the 1-smoothing of the $i$ th crossing will be part of two cycles, as seen in the following figure. One swaps the figures and rotates them for the case when the 0 -smoothing of the $i$ th crossing is part of two cycles.
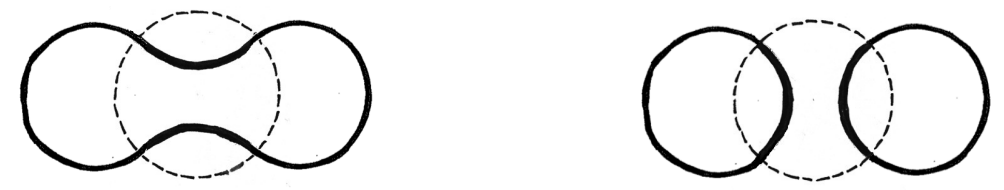

Therefore, $k_{\alpha}$ and $k_{\alpha^{\prime}}$ will differ by exactly one. When restricting to the cycles intersecting the crossing, each $d_{\zeta}$ will map either these two cycles to one cycle, or one cycle to two cycles. We want $d_{\zeta}$ to be the identity on the rest of the cycles. Each cycle is represented by $V$ in the chain complex, so these mappings are equivalent to finding graded linear maps that map $V \otimes V \rightarrow V$, and $V \rightarrow V \otimes V$.

Moreover, we require that $d_{\zeta}$ is a graded linear map, and thus is of homogeneous degree 0 (recall this means that the $q$-grading of a homogeneous element is unchanged under $\left.d_{\zeta}\right)$. First we must take into account the $q$-shift of each complex. The map $d_{\zeta}: V_{\alpha} \rightarrow V_{\alpha^{\prime}}$ maps $V^{\otimes k_{\alpha}}\left\{r_{\alpha}\right\} \rightarrow V^{\otimes k_{\alpha^{\prime}}}\left\{r_{\alpha}+1\right\}$, as $r_{\alpha^{\prime}}=r_{\alpha}+1$. Therefore, we require that the maps $V \otimes V \rightarrow V$ and $V \rightarrow V \otimes V$ to be of homogenous degree -1 (with respect to the $q$-grading).

These maps, known as $m$ and $\Delta$ are defined on the generators of $V$ and $V \otimes V$ as follows.

$$
\begin{aligned}
& m: \quad v_{+} \otimes v_{+} \mapsto v_{+} \\
& \Delta: \quad v_{+} \mapsto v_{+} \otimes v_{-}+v_{-} \otimes v_{+} \\
& v_{+} \otimes v_{-} \mapsto v_{-} \\
& v_{-} \mapsto v_{-} \otimes v_{-} \\
& v_{-} \otimes v_{+} \mapsto v_{-} \\
& v_{-} \otimes v_{-} \mapsto 0
\end{aligned}
$$

Note that these maps are homogenous of degree -1 , as required. Of course, $S_{\alpha}$ and $S_{\alpha^{\prime}}$ will be the same everywhere except at the cycles which are part of the $i$ th cross- 
ing. We want these other cycles to be unaffected by the graded linear map . Thus, the map $d_{\zeta}: V_{\alpha} \rightarrow V_{\alpha^{\prime}}$ will act as the identity on all other cycles in $S_{\alpha}$.

Let $\tau: V \otimes V \rightarrow V \otimes V$ be the twist map, $\tau(a \otimes b)=b \otimes a$. Note that since there is no natural ordering of the cycles of $S_{\alpha}$ (or the tensor product of cycles, $V^{\otimes k}$ ), this forces $m$ and $\Delta$ to be commutative $(m \tau=m)$ and co-commutative $(\tau \Delta=\Delta)$ respectively. So, $m\left(v_{+} \otimes v_{-}\right)=m\left(v_{-} \otimes v_{+}\right)$is forced, and also $\Delta\left(v_{+}\right)=v_{+} \otimes v_{-}+v_{-} \otimes v_{+}=$ $v_{-} \otimes v_{+}+v_{+} \otimes v_{-}=\tau \Delta\left(v_{+}\right)$is forced.

Example 4.2.3. The map $d_{0 \star 00}$ of the figure eight knot
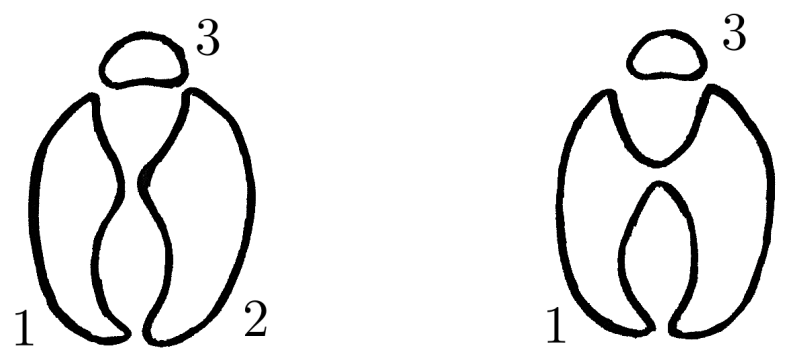

For convenience, I have labelled the cycles of the smoothings. We see that $d_{0 \star 00}$ leaves $V_{3}$ unchanged, and takes $V_{1} \otimes V_{2} \rightarrow V_{1}$. Since this is a 'merging' of two cycles which are labelled 1 and 2 , this happens via the map $m_{12}$, where the subscript refers to which cycles are to be merged. Similarly, $\mathrm{id}_{3}$ is the identity map on the cycle labelled 3. Therefore, the differential between the two maps is as follows.

$$
d_{00 \star 0}=m_{12} \otimes i d_{3}
$$

We now define the differentials of this chain complex, which are sums of the edges of the $n$-dimensional cube. We have to include a function which gives us the sign of 
the edges when we add them. The reason for this will be clear when we show that in fact these maps do form a cochain complex, $\llbracket D \rrbracket$.

\section{Definition 4.2.4. The differentials of the Khovanov bracket, $\llbracket \rrbracket$}

Let $Z_{n}$ be the collection of $\zeta \in\{0,1, \star\}^{n}$ where $\zeta$ contains exactly one $\star$. Recall $|\zeta|$ is the height of $\zeta$ (the number of 1 s in $\zeta$ ). Let $s:\{0,1, \star\}^{n} \rightarrow \mathbb{Z}^{\geq 0}$ be a function that maps each string of $\{0,1, \star\}^{n}$ to the number of 1 s on the left of the (leftmost) $\star$.

The $r$ th differential of the Khovanov cochain complex is as follows.

$$
d^{r}:=\sum_{\substack{|\zeta|=r \\ \zeta \in Z_{n}}}(-1)^{s(\zeta)} d_{\zeta}
$$

\section{Example 4.2.5. Differentials of the Hopf link}

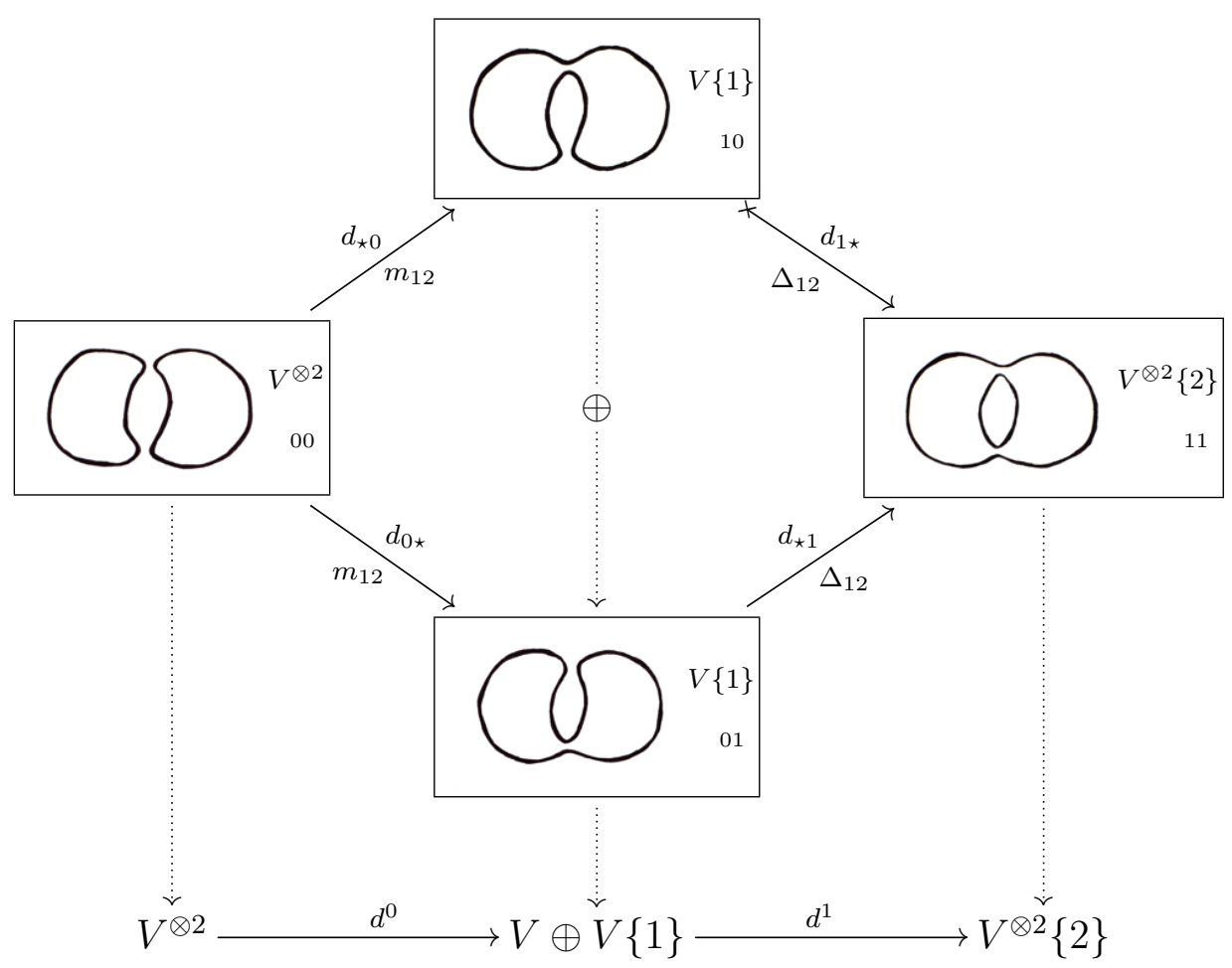


A negative differential is denoted by a cross on the tail of the arrow.

From the diagram, one can see the differentials are:

$$
\begin{gathered}
d^{0}=d_{\star 0}+d_{0 \star}=(m, m), \quad \text { hence for } v \in V_{00}, d^{0}(v)=(m v, m v) \in V_{10} \oplus V_{01}, \\
d^{1}=\left.d_{\star 1}\right|_{V_{10}}-\left.d_{1 \star}\right|_{V_{01}}=\Delta-\Delta, \quad \text { hence for }\left(v_{1}, v_{2}\right) \in V_{01} \oplus V_{10}, d^{1}\left(v_{1}, v_{2}\right)= \\
\Delta v_{1}-\Delta v_{2} \in V_{11} .
\end{gathered}
$$

Observe that the maps $\left\{d^{r}\right\}_{r \in \mathbb{Z}}$ form a well defined sequence of graded vector spaces on the Khovanov bracket as defined in Definition 4.2.2. That is, $d^{r}$ does indeed map $\llbracket D \rrbracket^{r} \rightarrow \llbracket D \rrbracket^{r+1}$, as each summand $d_{\zeta}$ of $d^{r}$ maps an element of height $r$ to an element of height $r+1$. Hence, it remains to show that this is in fact a chain complex, which we will discuss in the next lemma and theorem.

Lemma 4.2.6. If the faces of the n-dimensional cube commute without signs, then the Khovanov bracket is a cochain complex

Proof. We will prove that $(-1)^{s(\zeta)}$ in Equation 4.2 ensures that for every face of the $n$-dimensional cube, there will be an odd number of minus signs of the edges of this face. This means that if each face commutes without the minuses, they will anti-commute with the minuses. Thus, since composition $d^{r+1} \circ d^{r}$ is the same as summing over all the faces, we will be able to say the sums will cancel!

We now check that there will be an odd number of minus signs on each edge of the cube. The four edges of a face of a cube will be of the form $\alpha_{1} \star \alpha_{2} 0 \alpha_{3}$, $\alpha_{1} \star \alpha_{2} 1 \alpha_{3}, \alpha_{1} 0 \alpha_{2} \star \alpha_{3}$, and $\alpha_{1} 1 \alpha_{2} \star \alpha_{3}$ for some strings of $\{0,1\} \alpha_{1}, \alpha_{2}, \alpha_{3}$. Then $s\left(\alpha_{1} \star \alpha_{2} 0 \alpha_{3}\right)=s\left(\alpha_{1} \star \alpha_{2} 1 \alpha_{3}\right)=s\left(\alpha_{1} 0 \alpha_{2} \star \alpha_{3}\right)=-s\left(\alpha_{1} 1 \alpha_{2} \star \alpha_{3}\right)$.

Therefore, with Lemma 4.2.6, all we need to show that the Khovanov bracket is indeed a cochain complex is to prove that each face of the $n$-dimensional cube of 
smoothings commutes, which we state in the following theorem.

Theorem 4.2.7. Each face of the n-dimensional cube of smoothings commutes.

This proof is more straightforward using TQFTs. Hence, we prove this theorem in Section 7.3 of Chapter 7 .

We now show that the Khovanov bracket is invariant under the ordering of the crossings. We already have mentioned that the spaces of the Khovanov bracket are invariant under ordering of the crossings, but it remains to show that the Khovanov bracket is invariant up to isomorphism (as a chain complex) under ordering of the crossings. We first note that in general, the $n$-dimensional cube of two differently ordered knot diagrams are the same before considering the signs $(-1)^{s(\zeta)}$. Note that each edge of the cube depends only on the vertex at either edge. If one permutes the order of the crossings by $\sigma$, then the height of $\alpha$ is unchanged, $r_{\alpha}=r_{\sigma(\alpha)}$ (there is still the same the number of 1 -smoothings). Thus, the $d_{\zeta}$ will not change in the differential. However, the signs $(-1)^{s(\zeta)}$ change.

Proposition 4.2.8. The Khovanov bracket as defined in Definition 4.2.2 and Definition 4.2.4 is invariant of the ordering of the crossings (up to isomorphism).

Proof. Consider a knot diagram $D$ with $n$ crossings. Consider two orderings of the crossings of $D$, and let $\sigma$ be the permutation that changes the first ordering to the second ordering. We denote the chain complex with respect to the first ordering $\llbracket D \rrbracket$, and the chain complex with respect to the second ordering $\llbracket D \rrbracket_{\sigma}$. The graded vector spaces of the vertices of the $n$-dimensional cube of the first ordering of $D$ are simply denoted $V_{\alpha}$, and the vertices of the $n$-dimensional cube of the second ordering of $D$ are denoted $V_{\sigma(\alpha)}$. Similarly, $d_{\zeta}$ and $d_{\sigma(\zeta)}$ refer to the "same" edge map in each of the $n$-dimensional cube of smoothings. It is implicit that the sub-script $\sigma$ of these graded vector spaces and edge maps imply that these graded vector spaces refer to 
the second ordering, and those without $\sigma$ refer to the first ordering.

We will show that there is an isomorphism of chain complexes from $\llbracket D \rrbracket$ to $\llbracket D \rrbracket_{\sigma}$. Recall that with the ordering of the crossings as defined, the smoothing $S_{\alpha}$ is the same as the smoothing $S_{\sigma(\alpha)}$, hence there are isomorphisms between the graded vector spaces $\phi_{\alpha}: V_{\alpha} \rightarrow V_{\sigma(\alpha)}$. Note also that $-\phi_{\alpha}$ is also an isomorphism of graded vector spaces. We let $t$ be a function that depends on the string $\alpha$ and the permutation $\sigma$, which we will define properly in the next paragraph. For now, we define the $r$ th chain map component to be a sum $\sum_{\alpha=r}(-1)^{t(\alpha, \sigma)} \phi_{\alpha}$ which is an isomorphism of the $r$ th space of the chain complex.

We noted previously that changing the ordering of the crossings will change the differentials. More specifically, it changes the sign on the edges of the $n$-dimensional cube, or summands of the differential. That is, $s(\zeta)$ and $s(\sigma(\zeta))$ are not necessarily the same, therefore we require that the $(-1)^{t(\alpha, \sigma)}$ ensures that this collection of maps does in fact form chain map - that is, that they commute with the differentials.

We define $t(\alpha, \sigma)$ to be the number of transpositions of the 1s of $\alpha$ under $\sigma$ mod 2. In other words, we exclude all the 0 s from $\alpha$, and find the parity of the permutation of only the 1 s under $\sigma$ (the parity of a permutation is normally denoted $\operatorname{sgn}(\cdot)$ ). Recall that $s(\zeta)$ is the number of 1 s to the left of the $\star$.

Restricting our attention to an edge of the $n$-dimensional cube, we next show that the maps between the corresponding edges of the $n$-dimensional cube commute. Once we know this, we claim that the maps $\left\{\sum_{\alpha=r}(-1)^{t(\alpha, \sigma)} \phi_{\alpha}\right\}_{r \in \mathbb{Z}}$ form a chain complex map. This is because each edge map with minuses, $(-1)^{s(\zeta)} d_{\zeta}$, will commute with the $(-1)^{t(\alpha, \sigma)} \phi_{\alpha}$. Hence the sum of these maps from some $V_{\alpha}$, which is the the differential restricted to a summand of $\llbracket D \rrbracket^{r}$ and $\llbracket D \rrbracket_{\sigma}^{r}$ will commute with the $(-1)^{t(\alpha, \sigma)} \phi_{\alpha}$. Therefore, since this is true for each summand of $\llbracket D \rrbracket^{r}$, the (non-restricted) differential commutes with the maps $\sum_{\alpha=r}(-1)^{t(\alpha, \sigma)} \phi_{\alpha}$, as required for a chain map. More briefly, it is because of the universal property of the product and co-product of the finite direct sum that the edges $V_{\alpha} \rightarrow V_{\alpha^{\prime}}$ where $|\alpha|=r$ form a unique map $\llbracket D \rrbracket^{r} \rightarrow \llbracket D \rrbracket_{\sigma}^{r}$. 
Let $\alpha, \alpha^{\prime} \in\{0,1\}^{n}$ differ at only the $i$ th place, and let $\zeta$ be the edge of the $n$ dimensional cube between them, $\alpha \stackrel{\zeta}{\longrightarrow} \alpha^{\prime}$.

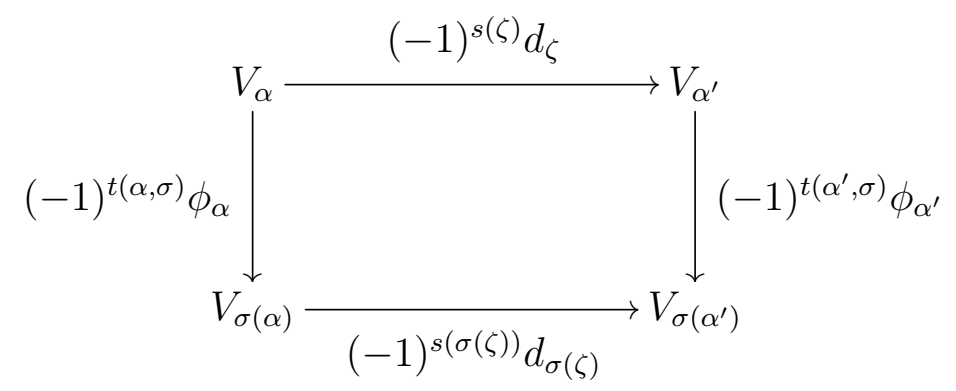

We know that $d_{\zeta}$ and $d_{\sigma(\zeta)}$ are the same in their respective $n$-dimensional cubes, hence $d_{\sigma(\zeta)} \phi_{\alpha}=\phi_{\alpha^{\prime}} d_{\zeta}$. What remains to show for the above diagram to commute is the following equality.

$$
s(\zeta)+t\left(\alpha^{\prime}, \sigma\right)=s(\sigma(\zeta))+t(\alpha, \sigma) \bmod 2
$$

Firstly, note $s(\zeta)-s(\sigma(\zeta))$ is the number of transpositions of $\star$ with the 1 s under $\sigma, \bmod 2$. Secondly, note $t(\alpha, \sigma)-t\left(\alpha^{\prime}, \sigma\right)$ is the number of times the $i$ th element of $\alpha$ is transposed with 1 s under $\sigma$, mod 2. Therefore, since $\star i s$ the $i$ th element of $\zeta, s(\zeta)-s(\sigma(\zeta))$ and $t(\alpha, \sigma)-t\left(\alpha^{\prime}, \sigma\right)$ are both the number of transpositions of the $i$ th element with respect to the 1 s under $\sigma(\bmod 2)$, so are in fact equal. Hence, the above equation holds and thus the diagram commutes, as each way around the diagram has the same number of minus signs.

Therefore, we have shown that there is an isomorphism of chain complexes between two Khovanov brackets of the same knot diagram under different ordering of crossings, as required.

Next, we will show that the Khovanov bracket as we have defined it satisfies Kh1, Kh2 and Kh3. The following proposition and proof are similar to the proposition and proof that the Kauffman bracket is well defined, but only when checking that the spaces of the cochain complex satisfy the three relations. This reinforces the 
point that the Khovanov bracket is constructed in an analogous way to the Kauffman bracket. In fact, like the Kauffman bracket, the relations Kh1, Kh2 and Kh3 define unique spaces of the chain complex (up to isomorphism). What makes matters more confusing in these definitions is the chain maps of the cochain complex. They do satisfy the three relations $\mathbf{K h} \mathbf{1}, \mathbf{K h} \mathbf{2}$ and $\mathbf{K h} \mathbf{3}$, but the three relations do not define unique chain maps of the Khovanov bracket.

Theorem 4.2.9. Let $D$ be a knot diagram. The chain complex $\llbracket D \rrbracket$ with the rth graded vector space as defined in Definition 4.2.2. and differentials as defined in Definition 4.2.4 satisfies the three Khovanov bracket relations $\mathbf{K h 1}, \boldsymbol{K h \mathbf { 2 }}, \boldsymbol{K h} \mathbf{3}$.

Proof. Recall that for any $K$-vector space $W$, that $W^{\otimes 0}:=K$. Consider the empty link, $\emptyset$. Then by definition, we get the following Khovanov bracket of the empty link.

$$
\llbracket \emptyset \rrbracket:=\left[\cdots \rightarrow 0 \rightarrow V^{\otimes 0} \rightarrow 0 \rightarrow \cdots\right]
$$

Hence, since $V^{\otimes 0}=K$, the relation $\mathbf{K h} \mathbf{1}$ is satisfied.

Consider a knot diagram $D$ and an unknot $\bigcirc$. Let $S_{\alpha}$ be a smoothing of $D$. Then the smoothing of $D \amalg \bigcirc$ corresponding to $\alpha$ is $S_{\alpha} \amalg \bigcirc$. Therefore, if we let $k_{\alpha}$ be the number of cycles in $S_{\alpha}$, then $k_{\alpha}+1$ will be the number of cycles in $S_{\alpha} \amalg \bigcirc$. The height of the smoothing of $D, r_{\alpha}$, will be the same as the height of the corresponding smoothing of $D \amalg \bigcirc$. Therefore, we have the following equalities for every $r$.

$$
\begin{aligned}
\llbracket D \amalg \bigcirc \rrbracket^{r} & =\bigoplus_{\substack{r_{\alpha}=r \\
\alpha \in\{0,1\}^{k}}} V^{\otimes\left(k_{\alpha}+1\right)}\left\{r_{\alpha}\right\} \\
& =\bigoplus_{\substack{r_{\alpha}=r \\
\alpha \in\{0,1\}^{k}}} V \otimes V^{\otimes k_{\alpha}}\left\{r_{\alpha}\right\} \\
& =V \otimes\left(\bigoplus_{\substack{r_{\alpha}=r \\
\alpha \in\{0,1\}^{k}}} V^{\otimes k_{\alpha}}\left\{r_{\alpha}\right\}\right) \\
& =V \otimes \llbracket D \rrbracket^{r}
\end{aligned}
$$


The summands of the differentials act as the identity on all cycles which are not part of the crossing in question. Hence if $d_{\zeta}$ is the $r$ th differential of $\llbracket D \rrbracket$, the $r$ th differential of $\llbracket D \amalg \bigcirc \rrbracket$ is $d_{\zeta} \otimes \mathrm{id}_{V}$, as required.

The relation Kh3 will be shown by induction on the number of crossings of the knot diagram $D$. It is clear for a knot $D$ with no crossings and $l$ components that $\llbracket D \rrbracket$ has the following components.

$$
\llbracket D \rrbracket^{r}= \begin{cases}V^{\otimes l} & : r=0 \\ 0 & : r \neq 0\end{cases}
$$

Consider a knot projection with $n=k$ crossings, $D$. For the induction hypothesis, we assume that the cochain complex $\llbracket D \rrbracket$ satisfies the Kh3. We now show that follows Kh3 is satisfied for a knot projection with $n=k+1$ crossings.

Consider a knot $D$ with $k+1$ crossings with an arbitrary numbering of the crossings. Choose the $i$ th crossing of $D$. Let $D_{0}$ be identical to $D$ but with a 0 -smoothing at the $i$ th crossing, and similarly $D_{1}$ be identical to $D$ but with a 1 -smoothing at the $i$ th crossing. We need to show that $\llbracket D \rrbracket$ as defined satisfies the relation $\mathbf{K h} \mathbf{3}$, that $\llbracket D \rrbracket=$ Flatten $\left[0 \rightarrow \llbracket D_{0} \rrbracket \rightarrow \llbracket D_{1} \rrbracket\{1\} \rightarrow 0\right]$. We will begin by showing that the spaces of $\llbracket D \rrbracket$ satisfy Kh3. Thus, we now show that the spaces of the chain complex $\llbracket D \rrbracket$ are as follows (as discussed in Section 4.1).

$$
\llbracket D \rrbracket^{r}= \begin{cases}\llbracket D_{0} \rrbracket^{r} & : r=0 \\ \llbracket D_{0} \rrbracket^{r} \oplus \llbracket D_{1} \rrbracket^{r-1}\{1\} & : 0<r<k+1 \\ \llbracket D_{1} \rrbracket^{r-1}\{1\} & : r=k+1\end{cases}
$$

First, we need to introduce some notation.

For $1 \leq j \leq n$, let $\alpha_{j}$ be the $j$ th element of the string $\alpha \in\{0,1\}^{n}$. For some $\alpha \in\{0,1\}^{n-1}$, let $\alpha^{0}$ and $\alpha^{1}$ represent an element of $\{0,1\}^{n}$ such that the $i$ th element in the string $\alpha^{0}$ is 0 , and $\left(\alpha^{0}\right)_{j<i}=\alpha_{j}$ and $\left(\alpha^{0}\right)_{j>i}=\alpha_{j-1}$. Similarly, the $i$ th element 
in the string $\alpha^{1}$ is 1 , and satisfies $\left(\alpha^{1}\right)_{j<i}=\alpha_{j}$ and $\left(\alpha^{1}\right)_{j>i}=\alpha_{j-1}$.

Then, where $k_{\alpha}^{0}$ is the number of cycles in $D_{0}$ with smoothing $\alpha$, and $k_{\alpha}^{1}$ is the number of cycles in $D_{1}$. Thus we have $k_{\alpha}^{0}=k_{\alpha^{0}}$ and $k_{\alpha}^{1}=k_{\alpha^{1}}$. Note that $r_{\alpha}$ depends only on $\alpha$, and not on the smoothing itself, and that $r_{\alpha^{0}}=r_{\alpha}=r_{\alpha^{1}}-1$.

$$
\begin{aligned}
\llbracket D \rrbracket^{r} & :=\bigoplus_{\substack{r_{\alpha}=r \\
\alpha \in\{0,1\}^{k+1}}} V^{\otimes k_{\alpha}}\left\{r_{\alpha}\right\} \\
& =\left(\bigoplus_{\substack{r_{\alpha 0}=r \\
\alpha^{0} \in\{0,1\}^{k+1}}} V^{\otimes k_{\alpha^{0}}}\left\{r_{\alpha^{0}}\right\}\right) \oplus\left(\bigoplus_{\substack{r_{\alpha 1}=r \\
\alpha^{1} \in\{0,1\}^{k+1}}} V^{\otimes k_{\alpha^{1}}}\left\{r_{\alpha^{1}}\right\}\right) \\
& =\left(\bigoplus_{\substack{r_{\alpha}=r \\
\alpha^{0} \in\{0,1\}^{k+1}}} V^{\otimes k_{\alpha^{0}}}\left\{r_{\alpha^{0}}\right\}\right) \oplus\left(\bigoplus_{\substack{r_{\alpha}-1=r-1 \\
\alpha^{1} \in\{0,1\}^{k+1}}}\left(V^{\otimes k_{\alpha^{1}}}\left\{r_{\alpha^{1}}-1\right\}\{1\}\right)\right) \\
& =\left(\bigoplus_{\substack{r_{\alpha}=r \\
\alpha \in\{0,1\}^{k}}} V^{\otimes k_{\alpha}^{0}}\left\{r_{\alpha}\right\}\right) \oplus\left(\left(\bigoplus_{\substack{r_{\alpha}=r-1 \\
\alpha \in\{0,1\}^{k}}} V^{\otimes k_{\alpha}^{1}}\left\{r_{\alpha}\right\}\right)\{1\}\right) \\
& =\llbracket D_{0} \rrbracket^{r} \oplus \llbracket D_{1} \rrbracket^{r-1}\{1\}
\end{aligned}
$$

The second equality is because $\left\{\alpha^{0}: \alpha \in\{0,1\}^{k}\right\} \cup\left\{\alpha^{1}: \alpha \in\{0,1\}^{k}\right\}=\{0,1\}^{k+1}$ and $\left\{\alpha^{0}: \alpha \in\{0,1\}^{k}\right\} \cap\left\{\alpha^{1}: \alpha \in\{0,1\}^{k}\right\}=\emptyset$. The above working is sufficient to say that the spaces as defined in Definition 4.2.2 satisfy Kh3.

Finally, we show that the differentials of $\llbracket D \rrbracket$ will also satisfy $\mathbf{K h} \mathbf{3}$, which we also show by induction on the number of crossings of $D$. Suppose $D$ has $n=1$ crossing, then there is one 0 -smoothing, $S_{0}$, and one 1-smoothing, $S_{1}$, of $D$. Let $k_{0}$ be the number of cycles in $S_{0}$ and $k_{1}$ be the number of cycles in $S_{1}$. Then the (only non-trivial) differential is defined to be either $m \otimes \mathrm{id}_{V^{\otimes\left(k_{0}-2\right)}}$ or $\Delta \otimes \mathrm{id}_{V^{\otimes\left(k_{0}-1\right)}}$. This is also the only nontrivial differential of the bi-complex constructed from $\left[0 \rightarrow \llbracket D_{0} \rrbracket \rightarrow \llbracket D_{1} \rrbracket\{1\} \rightarrow 0\right]$, and the only summand of this differential. Since the domain is $\llbracket D_{0} \rrbracket^{0},(-1)^{0}=1$, so there is no minus sign, as required. 
Now, for the induction step, consider a knot diagram $D$ with $n=k+1$ crossings. Let $D_{0}$ and $D_{1}$ be smoothings of $D$ which differ only at the $(k+1)$ th crossing (we choose this crossing for convenience). By definition, we know that the $r$ th differential of each $\llbracket D_{0} \rrbracket$ and $\llbracket D_{1} \rrbracket$ is $\sum_{|\zeta|=r}(-1)^{s(\zeta)} d_{\zeta}$ where $\zeta \in Z_{k}$. Although, in the notation with respect to the smoothings of $D$, these become $\sum_{|\zeta 0|=r}(-1)^{s(\zeta 0)} d_{\zeta 0}$ and $\sum_{|\zeta 1|=r}(-1)^{s(\zeta 1)} d_{\zeta 1}$ respectively. Note that $s(\zeta)=s(\zeta 0)=s(\zeta 1)$. Now we look at the differentials corresponding to the $(k+1)$ crossing, which we want to show are formed from the bi-complex below. That is, we want to show that $d^{i}=\sum_{p+q=i}\left(d_{v}^{p, q}+d_{h}^{p, q}\right)$ (see the beginning of this chapter for details about notation).

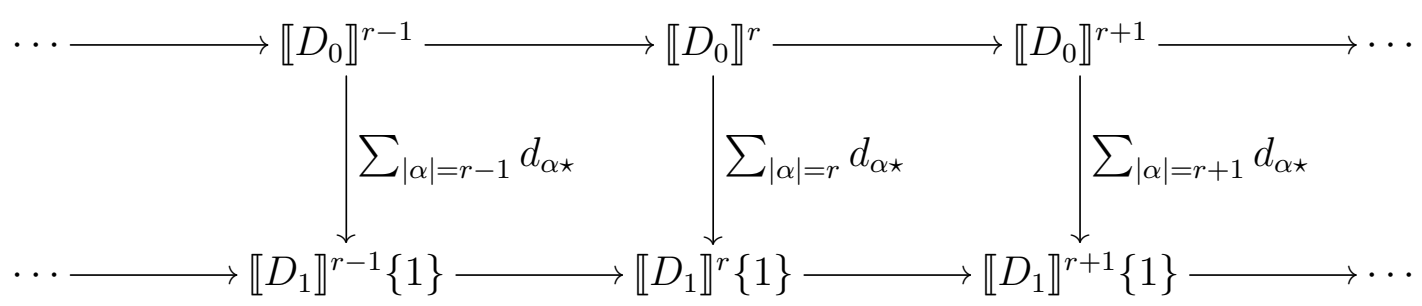

Hence, the $r$ th differential of $\llbracket D \rrbracket$ can be written as follows.

$$
\begin{aligned}
d^{r} & =\sum_{\substack{|\zeta|=r \\
\zeta \in Z_{k+1}}}(-1)^{s(\zeta)} d_{\zeta} \\
& =\sum_{\substack{|\zeta|=r \\
\zeta \in Z_{k}}}(-1)^{s(\zeta 0)} d_{\zeta 0}+\sum_{\substack{|\zeta|=r-1 \\
\zeta \in Z_{k}}}(-1)^{s(\zeta 1)} d_{\zeta 1}+\sum_{\substack{|\alpha|=r \\
\alpha \in\{0,1\}^{k}}}(-1)^{|\alpha|} d_{\alpha \star} \\
& =d_{0}^{r}+d_{1}^{r-1}+\sum_{\substack{|\alpha|=r \\
\alpha \in\{0,1\}^{k}}}(-1)^{|\alpha|} d_{\alpha \star}
\end{aligned}
$$

The second equality holds since $\left\{\zeta 0: \zeta \in Z_{k}\right\} \cup\left\{\zeta 1: \zeta \in Z_{k}\right\} \cup\left\{\alpha \star: \alpha \in\{0,1\}^{k}\right\}=$ $Z_{k+1}$ and $\left\{\zeta 0: \zeta \in Z_{k}\right\} \cap\left\{\zeta 1: \zeta \in Z_{k}\right\} \cap\left\{\alpha \star: \alpha \in\{0,1\}^{k}\right\}=\emptyset$. The final sum is exactly the $r$ th differential as defined from a bi-complex of the form above, as required. 


\subsection{The Khovanov cohomology}

Recall in Chapter 2, we first defined the Kauffman bracket on a knot diagram, and then defined the unnormalised Jones polynomial of an oriented knot diagram $D$ to be the Kauffman bracket of $D$ under the normalisation, $(-1)^{n_{-}} q^{n_{+}-2 n_{-}}$. Under this normalisation, we have a a knot invariant. Accordingly, to continue our analogous construction of the Khovanov chain complex with respect to the unnormalised Jones polynomial, we define the Khovanov chain complex to be the Khovanov bracket under a $q$-degree shift and a homological degree shift, $\left[-n_{-}\right]\left\{n_{+}-2 n_{-}\right\}$, as follows.

\section{Definition 4.3.1. The Khovanov chain complex}

The Khovanov cochain complex $\mathcal{C}^{*}(D)$ of an oriented knot diagram $D$ with $n_{+}$positive crossings and $n_{-}$negative crossings is defined as follows.

$$
\mathcal{C}^{*}(D):=\llbracket D \rrbracket\left[-n_{-}\right]\left\{n_{+}-2 n_{-}\right\}
$$

\section{Definition 4.3.2. The Khovanov cohomology}

The $r$ th Khovanov cohomology module, denoted $\mathrm{Kh}^{r}(D)$, is the cohomology of the cochain complex $\mathcal{C}^{*}(D)$.

Consider the $r$ th graded vector space of the Khovanov cochain complex, $\mathcal{C}^{r}(D)$. The vector space in $q$-degree $m$ of $\mathcal{C}^{r}(D)$ is denoted $\mathcal{C}_{m}^{r}(D)$. Similarly, the vector space in $q$-degree $m$ of the $r$ th cohomology module of the Khovanov cochain complex is denoted $\operatorname{Kh}_{m}^{r}(D)$. This follows the standard notation of both the homological degree of cochain complexes and the $q$ dim of graded vector spaces. Everything we have done in this section so far has been over a $\mathbb{Z}$-graded $K$-vector space. It all can be generalised to a $\mathbb{Z}$-graded $R$-module where $R$ is a commutative ring with identity. In fact, in Khovanov's original paper, the definition was over a polynomial ring $\mathbb{Z}[c]$. Such rings, particularly $\mathbb{Z}$, are interesting when applying the quotients (when finding the homology), as the interesting property of torsion sometimes arises. Unfortunately, this is beyond the scope of this thesis.

Finally, we state the fundamental property of the Khovanov homology. 
Theorem 4.3.3. The Khovanov homology is a knot invariant.

This theorem will proved in Chapter 5 and is mentioned again in Chapter 7 .

\subsection{Relation to Jones polynomial}

Proposition 4.4.1. The unnormalised Jones polynomial is the graded Euler characteristic of the Khovanov homology of an oriented knot D.

Proof. We know from Proposition 3.3 .13 that the graded Euler characteristic of a chain complex of finitely generated graded vector spaces $C^{*}$ is equal to the alternating sums of the graded dimensions of the chain complex $C^{*}$. Also, recall the Jones polynomial from Definition 2.2.5 is $\hat{J}(D)=(-1)^{n_{-}} q^{n_{+}-2 n_{-}}\langle D\rangle$. Using the properties of $q \mathrm{dim}$, the following equalities hold.

$$
\begin{aligned}
q \operatorname{dim}\left(\llbracket D \rrbracket^{r}\right) & =q \operatorname{dim}\left(\bigoplus_{r_{\alpha}=r} V_{\alpha}\right) \\
& =\sum_{r_{\alpha}=r} q \operatorname{dim}\left(V^{\otimes k_{\alpha}}\left\{r_{\alpha}\right\}\right) \\
& =\sum_{r_{\alpha}=r} q^{r_{\alpha}} q \operatorname{dim}\left(V^{\otimes k_{\alpha}}\right) \\
& =\sum_{r_{\alpha}=r} q^{r_{\alpha}}(q \operatorname{dim} V)^{k_{\alpha}} \\
& =\sum_{r_{\alpha}=r} q^{r_{\alpha}}\left(q+q^{-1}\right)^{k_{\alpha}}
\end{aligned}
$$

We must also consider the shift $\cdot\left[-n_{-}\right]\left\{n_{+}-2 n_{-}\right\}$. Recall that $q \operatorname{dim}(W\{l\})=$ $q^{l} q \operatorname{dim} W$. The shift $\left[-n_{-}\right]$will not affect the $q$-dimension, but will affect the power of $(-1)$ in the Euler characteristic by moving $i \mapsto i+n_{-}$. Therefore, we have the 
following.

$$
\begin{aligned}
q \operatorname{dim}\left(\mathcal{C}^{r}(D)\right) & =q \operatorname{dim}\left(\llbracket D \rrbracket^{r}\left[-n_{-}\right]\left\{n_{+}-2 n_{-}\right\}\right) \\
& =q^{n_{+}-2 n_{-}} q \operatorname{dim}\left(\llbracket D \rrbracket^{r+n_{-}}\right)
\end{aligned}
$$

Therefore, summing over all $r$, we find the Euler characteristic of $\mathcal{C}(D)$.

$$
\begin{aligned}
\chi_{q}\left(\mathcal{C}^{*}(D)\right) & :=\sum_{j}(-1)^{j} q \operatorname{dim}\left(\mathcal{C}^{j}(D)\right) \\
& =\sum_{j}(-1)^{j} q^{n_{+}-2 n_{-}} q \operatorname{dim}\left(\llbracket D \rrbracket^{j+n_{-}}\right) \\
& =\sum_{i}(-1)^{i-n_{-}} q^{n_{+}-2 n_{-}} q \operatorname{dim}\left(\llbracket D \rrbracket^{i}\right) \\
& =(-1)^{n_{-}} q^{n_{+}-2 n_{-}} \sum_{i}(-1)^{i}\left(\sum_{r_{\alpha}=i} q^{r_{\alpha}}\left(q+q^{-1}\right)^{k_{\alpha}}\right) \\
& =(-1)^{n_{-}} q^{n_{+}-2 n_{-}} \sum_{\alpha \in\{0,1\}^{n}}(-1)^{r_{\alpha}}\left(q^{r_{\alpha}}\left(q+q^{-1}\right)^{k_{\alpha}}\right) \\
& =(-1)^{n_{-}} q^{n_{+}-2 n_{-}}\langle D\rangle
\end{aligned}
$$

The fourth equality is summing over all of $\alpha \in\{0,1\}^{n}$. In other words, $\bigcup_{i \in \mathbb{Z}}\{\alpha \in$ $\left.\{0,1\}^{n}: r_{\alpha}=i\right\}=\{0,1\}^{n}$. The last line is the unnormalised Jones polynomial of $D$, as required.

Khovanov homology is a strictly stronger invariant than the Jones polynomial. We have shown that the Khovanov homology encodes the Jones polynomial, hence if two knots have the same Khovanov homology, they will have the same Jones polynomial. The following is an example of a two knots, $5_{1}$ and $10_{132}$, that have the same Jones polynomial but different Khovanov homologies, which shows it is indeed a stronger invariant. These were shown to have different Khovanov homologies in [1]. 

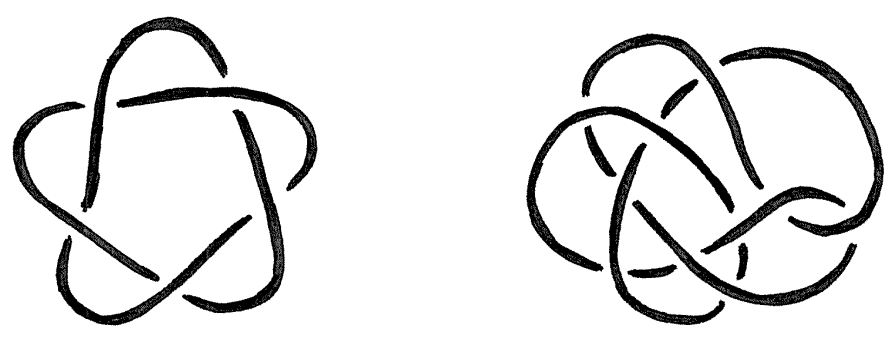

An incredibly appealing property of the Khovanov homology is that it is an unknotdetector, [8]. That is, if a knot diagram $D$ has the same Khovanov homology as the unknot, then the knot diagram $D$ is the unknot. Instead, while it is unknown that the Jones is an unknot-detector for knots with only one component, there are knots with more than one component, or links, that have the same Jones polynomial as the unknot.

\subsection{A long exact sequence}

A nice feature of attributing a cochain complex to every knot diagram that one can use properties of homological algebra. In particular, we construct a short exact sequence of chain complexes, and then use Theorem 3.2.8 in Chapter 3 to construct a long exact sequence of homology graded vector spaces. In this case, each long exact sequence is based on one particular crossing of a knot, and in the following proposition we will consider a positive crossing. The long exact sequence of a negative crossing can be obtained similarly with some modifications of the indices. This follows [13].

\section{Theorem 4.5.1. Long exact sequence of a knot}

Let $D$ be an oriented knot diagram with a positive crossing. Let $D_{0}$ and $D_{1}$ be knot diagrams where the positive crossing is replaced with either a 0 -smoothing or 1-smoothing respectively. Then there is a long exact sequence of Khovanov homology modules:

$$
\cdots \rightarrow K h_{j-3 c-2}^{i-c-1}\left(D_{1}\right) \rightarrow K h_{j}^{i}(D) \rightarrow K h_{j-1}^{i}\left(D_{0}\right) \rightarrow K h_{j-3 c-2}^{i-c}\left(D_{1}\right) \rightarrow \cdots
$$


where $c:=\left(\right.$ number of negative crossings in $\left.D_{1}\right)-($ number of negative crossings in $D)$.

Proof. The construction of the short exact sequence follows from the construction of the Khovanov bracket.

Suppose $D$ is a knot diagram with $n$ crossings. Let $d_{0}^{r}$ represent the $r$ th differential of $\llbracket D_{0} \rrbracket$ and $d_{1}^{r}$ represent the $r$ th differential of $\llbracket D_{1} \rrbracket$. Recall the spaces of $\llbracket D \rrbracket$ are as follows.

$$
\llbracket D \rrbracket^{r}= \begin{cases}\llbracket D_{0} \rrbracket^{r} & : r=0 \\ \llbracket D_{0} \rrbracket^{r} \oplus \llbracket D_{1} \rrbracket^{r-1}\{1\} & : 0<r<n \\ \llbracket D_{1} \rrbracket^{r-1}\{1\} & : r=n\end{cases}
$$

Hence, $\mathcal{C}^{r}(D)$ can be written as a direct sum of $\mathcal{C}^{r}\left(D_{0}\right)$ and $\mathcal{C}^{r-1}\left(D_{1}\right)$ under some shifts in the homological grading and the $q$-grading, which makes up the bulk of this proof. Nonetheless, we first must construct a short exact sequence of the brackets.

We claim that $\iota^{i}: \llbracket D_{1} \rrbracket^{i-1}\{1\} \hookrightarrow \llbracket D_{0} \rrbracket^{i} \oplus \llbracket D_{1} \rrbracket^{i-1}\{1\}$ is an injective chain map. We need only to check $\iota$ commutes with the differentials. This can be seen because each differential of $\llbracket D \rrbracket$ will be a sum of the edges of the $n$-dimensional cube of $D_{0}$, $D_{1}$, or from $D_{0}$ to $D_{1}$, since each summand of the differential replaces a 0 -smoothing with a 1 -smoothing. Therefore the restriction of the $i$ th differential to $\llbracket D_{1} \rrbracket^{i-1}\{1\}$ in $\llbracket D_{0} \rrbracket^{i} \oplus \llbracket D_{1} \rrbracket^{i-1}\{1\}$ is exactly the $i$ th differential in the chain complex $\llbracket D_{1} \rrbracket^{i-1}\{1\}$.

Next, we claim the surjective projection map $j^{i}: \llbracket D_{0} \rrbracket^{i} \oplus \llbracket D_{1} \rrbracket^{i-1}\{1\} \rightarrow \llbracket D_{0} \rrbracket^{i}$ is a chain map. This can be seen for similar reasons as above. Also, it is clear that the image of $\iota$ is exactly the kernel of $\jmath$. Hence, we have the following exact sequence of cochain complexes.

$$
0^{*} \rightarrow \llbracket D_{1} \rrbracket[1]\{1\} \hookrightarrow \llbracket D \rrbracket \rightarrow \llbracket D_{0} \rrbracket \rightarrow 0^{*}
$$

As mentioned in Subsection 3.2.2, we know that this is an exact sequence of graded 
vector spaces for each homological degree. Since the maps between graded vector spaces graded linear maps (they preserve the grading), we know when restricting to a particular $q$-grading, we have an exact sequence of vector spaces. Hence, for every $(i, j)$ (where $i$ is the homological degree and $j$ is the $q$-degree), we have the following exact sequence of vector spaces.

$$
0 \rightarrow \llbracket D_{1} \rrbracket_{j-1}^{i-1} \hookrightarrow \llbracket D \rrbracket_{j}^{i} \rightarrow \llbracket D_{0} \rrbracket_{j}^{i} \rightarrow 0
$$

From this form, we alter the indices under the normalisation $\cdot\left[-n_{-}\right]\left\{n_{+}-2 n_{-}\right\}$to find a short exact sequence of the Khovanov chain complexes of $D, D_{0}, D_{1}$.

Since the normalisation of the Khovanov bracket depends on how the knot is oriented, we must consider separately when the crossing in question is positive or negative. We will consider the case where the crossing is positive.

In the case the crossing is positive, $D_{0}$ will inherit the orientation of $D$.
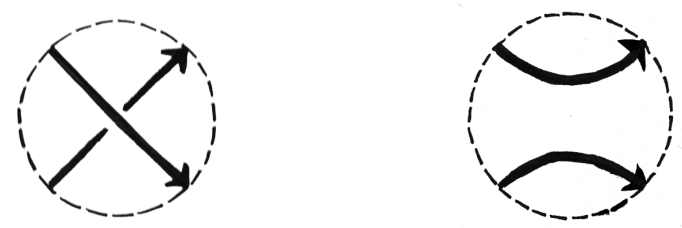

Instead, for $D_{1}$, one must arbitrarily choose the orientation. Define $c \in \mathbb{Z}$ as follows.

$c=$ number of negative crossings in $D_{1}$ - number of negative crossings in $D$

Therefore we can we find the Khovanov chain complex of $D, D_{0}$, and $D_{1}$.

1. $D$ has $n_{+}$positive crossings and $n_{-}$negative crossings, so $\mathcal{C}^{*}(D)=\llbracket D \rrbracket\left[-n_{-}\right]\left\{n_{+}-\right.$ $\left.2 n_{-}\right\}$,

2. $D_{0}$ has $n_{+}-1$ positive crossings and $n_{-}$negative crossings, so $\mathcal{C}^{*}\left(D_{0}\right)=$ $\llbracket D_{0} \rrbracket\left[-n_{-}\right]\left\{n_{+}-1-2 n_{-}\right\}$, 
3. $D_{1}$ has $\left(n_{+}+n_{-}\right)-\left(c+n_{-}\right)-1=n_{+}-c-1$ positive crossings and $c+n_{-}$ negative crossings, so $\mathcal{C}^{*}\left(D_{1}\right)=\llbracket D_{1} \rrbracket\left[-\left(c+n_{-}\right)\right]\left\{n_{+}-c-1-2\left(c+n_{-}\right)\right\}=$ $\llbracket D_{1} \rrbracket\left[-c-n_{-}\right]\left\{n_{+}-2 n_{-}-3 c-1\right\}$.

Note also that $\mathcal{C}_{j}^{i}[a]\{b\}=\mathcal{C}_{j-b}^{i-a}$, so $\mathcal{C}_{j+b}^{i+a}[a]\{b\}=\mathcal{C}_{j}^{i}$.

We now write each of the graded vector spaces in equation 4.3 after the shift $\cdot\left[-n_{-}\right]\left\{n_{+}-\right.$ $\left.2 n_{-}\right\}$.

$$
\begin{aligned}
& \llbracket D_{1} \rrbracket_{j-1}^{i-1}\left[-n_{-}\right]\left\{n_{+}-2 n_{-}\right\}=\llbracket D_{1} \rrbracket_{j-1-3 c-1}^{i-c-1}\left[-n_{-}-c\right]\left\{n_{+}-2 n_{-}-3 c-1\right\} \\
& =\mathcal{C}_{j-3 c-2}^{i-c-1}\left(D_{1}\right) \\
& \llbracket D \rrbracket_{j}^{i}\left[-n_{-}\right]\left\{n_{+}-2 n_{-}\right\}=\llbracket D \rrbracket_{j}^{i}\left[-n_{-}\right]\left\{n_{+}-2 n_{-}\right\} \\
& =\mathcal{C}_{j}^{i}(D) \\
& \llbracket D_{0} \rrbracket_{j}^{i}\left[-n_{-}\right]\left\{n_{+}-2 n_{-}\right\}=\llbracket D_{0} \rrbracket_{j-1}^{i}\left[-n_{-}\right]\left\{n_{+}-2 n_{-}-1\right\} \\
& =\mathcal{C}_{j-1}^{i}\left(D_{0}\right)
\end{aligned}
$$

Hence we have the following exact chain complex of graded vector spaces.

$$
0 \rightarrow \mathcal{C}_{j-3 c-2}^{i-c-1}\left(D_{1}\right) \rightarrow \mathcal{C}_{j}^{i}(D) \rightarrow \mathcal{C}_{j-1}^{i}\left(D_{0}\right) \rightarrow 0
$$

Since all the chain maps are unaffected by the shifts, we have the following short exact sequence of chain complexes. After this, we apply Theorem 3.2 .8 to get a long exact sequence of vector spaces (not graded - but it is easy enough to form the graded vector space by "combining" the vector spaces).

$$
0^{*} \rightarrow \mathcal{C}_{j-3 c-2}^{*}\left(D_{1}\right)[c+1] \rightarrow \mathcal{C}_{j}^{*}(D) \rightarrow \mathcal{C}_{j-1}^{*}\left(D_{0}\right) \rightarrow 0^{*}
$$

Recall the homological shifts of the cochain complexes also shift the homology in a 
similar way. Therefore, this short exact sequence gives us the long exact sequence of Khovanov homologies for each $q$-degree $j \in \mathbb{Z}$ as follows.

$$
\cdots \rightarrow \mathrm{Kh}_{j-3 c-2}^{i-c-1}\left(D_{1}\right) \rightarrow \mathrm{Kh}_{j}^{i}(D) \rightarrow \mathrm{Kh}_{j-1}^{i}\left(D_{0}\right) \rightarrow \mathrm{Kh}_{j-3 c-2}^{i-c}\left(D_{1}\right) \rightarrow \cdots
$$

As required. 


\section{Chapter 5}

\section{Invariance under the Reidemeister moves}

In this chapter, we follow Dror Bar-Natan's proofs of the Reidemeister invariance of the Khovanov homology in [1]. We endeavour to include all details. These proofs only use algebraic techniques to show that two complexes have the same homologies. In the following proof of $\mathbf{R} \mathbf{1}$ and $\mathbf{R} \mathbf{2}$, the techniques are analogous to the proof of the Jones polynomial. However, in the proof of invariance under R3, where one uses R2, one requires a more sophisticated method.

We will use the cone construction, which is described in Chapter 3 . Specifically, for $\mathbf{R} 1$, we show that $\llbracket \Omega \rrbracket=$ Cone $[\llbracket \bigcirc \rrbracket \rightarrow \llbracket \Omega \rrbracket]$, which we describe soon. It is useful to first state and prove the following lemma.

\section{Lemma 5.0.2. Cancellation theorems}

Let $\mathcal{C}, \mathcal{C}^{\prime}$ be chain complexes, where $\mathcal{C}^{\prime}$ is a sub-complex of $\mathcal{C}$.

1. If $\mathcal{C}^{\prime}$ is acyclic, then $H^{n}(\mathcal{C}) \cong H^{n}\left(\mathcal{C} / \mathcal{C}^{\prime}\right)$ for every $n$,

2. If $\mathcal{C} / \mathcal{C}^{\prime}$ is acyclic, then $H^{n}(\mathcal{C}) \cong H^{n}\left(\mathcal{C}^{\prime}\right)$ for every $n$.

Proof. 1. Let $\mathcal{C}^{\prime}$ be acyclic, and consider the short exact sequence of chain complexes

$$
0 \rightarrow \mathcal{C}^{\prime} \hookrightarrow \mathcal{C} \rightarrow \mathcal{C} / \mathcal{C}^{\prime} \rightarrow 0
$$


This induces a long exact sequence of homology modules

$$
\cdots \rightarrow H^{n-1}\left(\mathcal{C} / \mathcal{C}^{\prime}\right) \rightarrow H^{n}\left(\mathcal{C}^{\prime}\right) \rightarrow H^{n}(\mathcal{C}) \rightarrow H^{n}\left(\mathcal{C} / \mathcal{C}^{\prime}\right) \rightarrow H^{n+1}\left(\mathcal{C}^{\prime}\right) \rightarrow \cdots
$$

By assumption, $H^{n}\left(\mathcal{C}^{\prime}\right)=0$ for every $n$, so the map $H^{n}(\mathcal{C}) \rightarrow H^{n}\left(\mathcal{C} / \mathcal{C}^{\prime}\right)$ is both an injection by exactness at $H^{n}(\mathcal{C})$, and surjective by exactness at $H^{n}\left(\mathcal{C} / \mathcal{C}^{\prime}\right)$. Therefore, we have isomorphisms $H^{n}(\mathcal{C}) \cong H^{n}\left(\mathcal{C} / \mathcal{C}^{\prime}\right)$ for every $n$, so $\mathcal{C}$ and $\mathcal{C} / \mathcal{C}^{\prime}$ are quasi-isomorphic.

2. Let $\mathcal{C} / \mathcal{C}^{\prime}$ be acyclic, then $H^{n}(\mathcal{C}) \cong H^{n}\left(\mathcal{C}^{\prime}\right)$ for every $n$. Similarly we find from the long exact sequence that $H^{n}(\mathcal{C}) \rightarrow H^{n}\left(\mathcal{C}^{\prime}\right)$ must be injective and surjective for every $n$, and thecoore $\mathcal{C}$ and $\mathcal{C}^{\prime}$ are quasi-isomorphic.

\section{Notation, cochain complexes, and quotients}

Firstly, we let the concatenation of two strings, say $\alpha$ and $\beta$, simply be written as their juxtaposition $\alpha \beta$. Hence, for $\alpha \in\{0,1\}^{n-1}$, and if $S, S_{0}$ and $S_{1}$ denote smoothings of $D, D_{0}$ and $D_{1}$ which differ in the first crossing respectively, then conveniently our notation implies $\left(S_{0}\right)_{\alpha}=S_{0 \alpha},\left(S_{1}\right)_{\alpha}=S_{1 \alpha},\left(V_{0}\right)_{\alpha}=V_{0 \alpha}$ and $\left(V_{1}\right)_{\alpha}=V_{1 \alpha}$.

Next, we briefly describe an approach to view the constructions we use. Let $D$ be a knot diagram with $n$ crossings. Let $D_{0}$ be the same knot diagram as $D$ with a 0 -smoothing in the first crossing and similarly let $D_{1}$ be the same knot diagram as $D$ with a 1-smoothing in the first crossing. Recall each edge of the $n$-dimensional cube is the graded vector space denoted by $V_{0 \alpha}$ or $V_{1 \alpha}$, which are vertices of the $(n-1)$ dimensional cube of $D_{0}$ and $D_{1}$ respectively. It is useful to discuss maps between the "sub"-n-dimensional cubes of a knot diagram $D$, which is defined by the collection of maps as follows.

$$
\left\{\left[d_{\star \alpha}: V_{0 \alpha} \longrightarrow V_{1 \alpha}\right]: \alpha \in\{0,1\}^{n-1}\right\}
$$

We have chosen $D_{0}$ and $D_{1}$ to be smoothings of the first crossing of $D$ to simplify things in terms of the minus signs, which we can assume since we showed in Chapter 
4 that the Khovanov bracket doesn't depend on the ordering of the smoothings in the knot diagram.

Hence, in the map between the $r$ th spaces of Khovanov brackets is sum $\sum_{|\alpha|=r} d_{\star \alpha}$. Note that these maps will not form a chain map between the brackets $\llbracket D_{0} \rrbracket$ and $\llbracket D_{1} \rrbracket$, since (put briefly) the faces including the $d_{\star \alpha}$ are required to anti-commute.

So, for this chapter, when we talk of the "complex,"

$$
\left[\llbracket D_{0} \rrbracket \longrightarrow \llbracket D_{1} \rrbracket\right]
$$

which is a cochain complex of cochain complexes, we are implicitly flattening this complex - that is, putting it in a bi-complex and then taking the direct sums of the diagonals. Note in this case, to form a a bi-complex which matches the definitions of the differentials in Chapter 4 , we choose minuses in front of all edges of all differentials in $D_{1}$, that is, if $\zeta \in Z_{n-1}$ then $-(-1)^{s(\zeta)}\left(d_{1}\right)_{\zeta}=(-1)^{s(1 \zeta)} d_{1 \zeta}$ since $s(1 \zeta)=s(\zeta)+1$.

Now we talk briefly of taking quotients. All the following proofs rely heavily on taking quotients of cochain complexes in the form above, and using Lemma 5.0.2. We now show that this is indeed is well defined.

Let $\left[B^{*} \stackrel{f^{*}}{\longrightarrow} C^{*}\right]$ be a cochain complex as described above, and note that this is in fact the cone of $f^{*}$. We claim that it is sufficient to show that if $B^{\prime *}, C^{* *}$ are sub-complexes of $B^{*}$ and $C^{*}$ respectively, and the map $f^{\prime *}: B^{\prime *} \rightarrow C^{*}$ is a restriction of the chain map $f^{*}: B^{*} \rightarrow C^{*}$, then $\left[B^{\prime *} \stackrel{f^{\prime *}}{\longrightarrow} C^{\prime *}\right]$ is indeed a sub-complex. This can be done by considering the cone construction, and then follows rather effortlessly.

$$
\left(\begin{array}{cc}
d_{B^{\prime}}^{n+1} & 0 \\
f^{\prime n+1} & -d_{C^{\prime}}^{n}
\end{array}\right)\left(\begin{array}{cc}
d_{B^{\prime}}^{n} & 0 \\
f^{\prime n} & -d_{C^{\prime}}^{n-1}
\end{array}\right)=\left(\begin{array}{cc}
d_{B^{\prime}}^{n+1} d_{B^{\prime}}^{n} & 0 \\
f^{\prime n+1} d_{B^{\prime}}^{n}-d_{C^{\prime}}^{n} f^{\prime n} & d_{C^{\prime}}^{n} d_{C^{\prime}}^{n-1}
\end{array}\right)=\left(\begin{array}{ll}
0 & 0 \\
0 & 0
\end{array}\right)
$$

We add that $d_{B^{\prime}}^{n+1} d_{B^{\prime}}^{n}=d_{C^{\prime}}^{n} d_{C^{\prime}}^{n-1}=0$ follows since $B^{\prime *}, C^{* *}$ are chain complexes. Next, $f^{\prime n+1} d_{B^{\prime}}^{n}-d_{C^{\prime}}^{n} f^{\prime n}=0$ follows since $f^{\prime n+1} d_{B^{\prime}}^{n}=\left.f^{n+1} d_{B}^{n}\right|_{B^{\prime}}=\left.d_{C}^{n} f^{n}\right|_{B^{\prime}}=d_{C^{\prime}}^{n} f^{\prime n}$.

What remains is to show that putting a cochain complex map into a bi-complex 
and then taking direct sums of the diagonals is the same as taking the cone. This is simple if one puts the minuses in the right places for the bi-complex, which in this example is on the row or column containing the differentials of $C^{*}, d_{C}$. With no further comment, we continue to the proofs.

\subsection{First Reidemeister move}

In this section, we will discuss the proof of the first Reidemeister move using geometric complexes for the Khovanov homology of graded vector spaces. Let $D$ denote , and $D^{\prime}$ denote $\Omega$. The opposite orientation is similar, as seen the proof of Theorem 2.2.6. Additionally, we let $D_{0}$ be the 0 -smoothing of $D, \stackrel{\bigcirc}{\bigcirc}$, and let $D_{1}$ be the 1-smoothing, $\Omega$. Also, let $n, n_{+}$and $n_{-}$be the number of crossings, positive crossings and negative crossings of $D$, respectively. Without loss of generality, we assume the crossing in is the first crossing, and that the ordering of the crossings of $D_{0}$ and $D_{1}$ is inherited from $D$.

We will show that the Khovanov homologies of $D$ and $D^{\prime}$ are the same by rewriting the Khovanov bracket of $D$ in terms of the Khovanov brackets of $D_{0}$ and $D_{1}$, which will we discuss in detail. The cancellation theorems are used to find a "simpler" complex which is quasi-isomorphic to $D$, and also has a more evident isomorphism with $D^{\prime}$. Lastly, we show that the degree shifts to construct the quasi-isomorphism are adjusted for under the shift map (that is, we use that two quasi-isomorphic complexes are still quasi-isomorphic under the same shift of the $q$-degree, $\{\cdot\}$, and the same shift of the homological degree, $[\cdot])$.

Proof of invariance of the Khovanov homology under $\boldsymbol{R} \mathbf{1}$. We start by showing that the Khovanov bracket $\llbracket \Omega \rrbracket$ can be rewritten as Cone $\left(\llbracket \bigcirc \rrbracket^{*} \stackrel{\breve{m}^{*}}{\longrightarrow} \llbracket \Omega \rrbracket^{*-1}\right)$.

Let $d_{0}$ and $d_{1}$ be the differentials of $\llbracket \rrbracket^{*}$ and $\llbracket \Omega \rrbracket^{*-1}$ respectively, where a superscript will denote the degree of the differential in their respective cochain complexes. 
From Equation 4.2 , the differentials of $\llbracket \Omega$ can be written as follows.

$$
\begin{aligned}
d^{r} & :=\sum_{|\zeta|=r}(-1)^{s(\zeta)} d_{\zeta} \\
& =\sum_{|\zeta|=r}(-1)^{s(0 \zeta)} d_{0 \zeta}+\sum_{|\zeta|=r-1}(-1)^{s(1 \zeta)} d_{1 \zeta}+\sum_{|\alpha|=r}(-1)^{s(\star \alpha)} d_{\star \alpha} \\
& =d_{0}^{r}-d_{1}^{r-1}+\sum_{|\alpha|=r} d_{\star \alpha}
\end{aligned}
$$

Here we use $s(\star \alpha)=0$ and $s(1 \zeta)=s(\zeta)+1$. In the following working, we write $d^{r+1} d^{r}$ in terms of the above. Needless to say, composition of the differentials only occurs where there is well defined composition of maps.

$$
\begin{aligned}
d^{r+1} d^{r} & =\left(d_{0}^{r+1}-d_{1}^{r}+\sum_{|\alpha|=r+1} d_{\star \alpha}\right)\left(d_{0}^{r}-d_{1}^{r-1}+\sum_{|\alpha|=r} d_{\star \alpha}\right) \\
& =d_{0}^{r+1} d_{0}^{r}+d_{1}^{r} d_{1}^{r-1}+\left(\sum_{|\alpha|=r+1} d_{\star \alpha}\right)\left(\sum_{|\alpha|=r} d_{\star \alpha}\right)+\left(\sum_{|\alpha|=r+1} d_{\star \alpha}\right) d_{0}^{r}-d_{1}^{r}\left(\sum_{|\alpha|=r} d_{\star \alpha}\right) \\
& =\left(\sum_{|\alpha|=r+1} d_{\star \alpha}\right) d_{0}^{r}-d_{1}^{r}\left(\sum_{|\alpha|=r} d_{\star \alpha}\right)
\end{aligned}
$$

Since these are differentials of a cochain complex, $d^{r+1} d^{r}=0$, so the last line is equal to zero. Hence, when the Khovanov bracket is drawn as the following diagram, we use the above equality to assert that the vertical maps in the following diagram form a chain complex map.

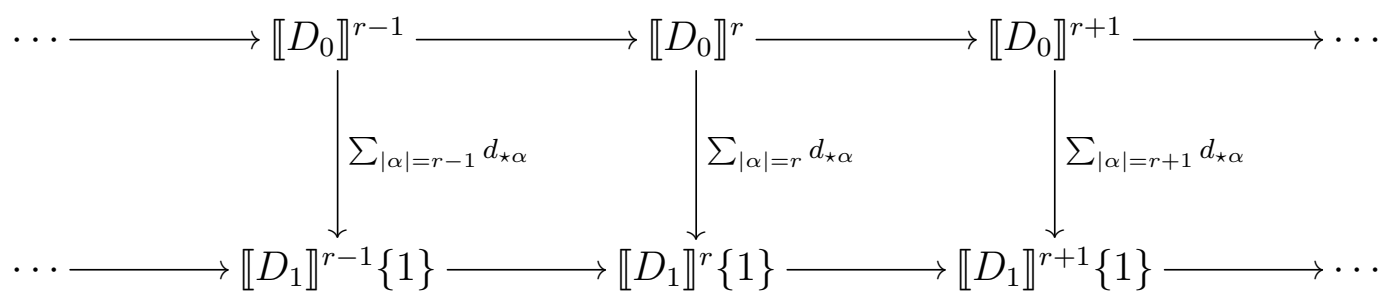

Next, we note that each summand of the vertical chain maps is a map from a smooth- 
ing of $\bigcirc$ to $\Omega$, and hence each summand is the map $m \otimes \mathrm{id}$, where $m$ acts on the two cycles seen in $\bigcirc$. We denote the collection of these maps that form the chain complex by $\breve{m}^{*}$. That is, it is the edge map which is the identity on all cycles

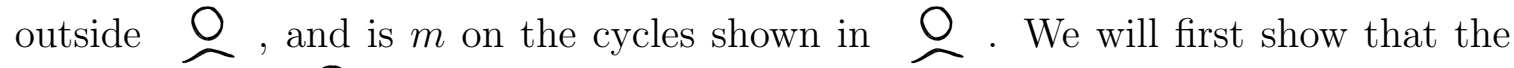
chain complex $\llbracket \Omega$ can be written as $\operatorname{Cone}\left(\breve{m}^{*}\right)$.

Every element of $\llbracket D \rrbracket^{r}$ can be written as a unique sum $a+b$ where $a \in \llbracket D_{0} \rrbracket^{r}$ and $b \in \llbracket D_{1} \rrbracket^{r-1}$ (a property of the direct sum). Hence, as the differential shown above, $d^{r}(a+b)=d^{r}(a)+(\breve{m})^{r}(a)-d^{r-1}(b)$.

We claim that $\llbracket \Omega \rrbracket=$ Cone $\left(\llbracket \bigcirc \rrbracket^{*} \stackrel{\breve{m}^{*}}{\rightarrow} \llbracket \Omega \rrbracket^{*-1}\right)$, so each $\llbracket \Omega \rrbracket^{r}$ is the direct sum $\llbracket \rrbracket^{r} \oplus \llbracket \Omega \rrbracket^{r-1}$. We now will define the differentials in the context of these direct sums (and from the equation above follows almost tautologically). We now will write this differential as a matrix to act on the ordered pairs from the direct sum.

$$
\left(\begin{array}{cc}
d_{0}^{n} & 0 \\
\breve{m} & -d_{1}^{n-1}
\end{array}\right)
$$

Note that the minus signs are to ensure the differential is the same as in $\Omega$. The following shows that the differential here is indeed the same as that in $\llbracket$.

$$
(a, b) \mapsto\left(d_{0}(a), \breve{m}(a)-d_{1}(b)\right)
$$

Recall that $m$ is the merging map $V \otimes V \rightarrow V$. It is defined on the generators of $V \otimes V$ as follows.

$$
\begin{aligned}
m: \quad v_{+} \otimes v_{+} & \mapsto v_{+} \\
v_{+} \otimes v_{-}, v_{-} \otimes v_{+} & \mapsto v_{-} \\
v_{-} \otimes v_{-} & \mapsto 0
\end{aligned}
$$

Consider the complex $\because \underset{\bigcirc}{\bigcirc} \rrbracket_{v_{+}}^{*} \oplus \llbracket \Omega \rrbracket^{*-1}$, where the subscript " $v_{+}$" denotes that

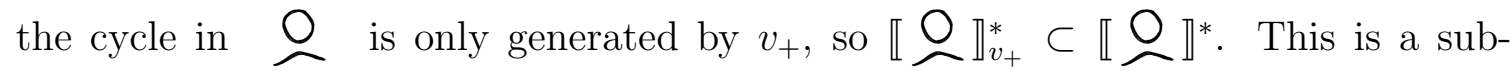


complex of $\llbracket \rrbracket^{*} \oplus \llbracket \Omega \rrbracket^{*-1}$ since the cycle in $\bigcirc$ will be unaffected by the differentials, and thus the differentials act as the identity on $v_{+}$.

We now will show that this complex is acyclic by showing every $r$-cocycle of $\llbracket \stackrel{\bigcirc}{\bigcirc} \rrbracket_{v_{+}}^{*} \oplus$ $\llbracket \Omega \rrbracket^{*-1}$ is also an $r$-coboundary. In other words, we show that if $(a, b) \in \llbracket \bigcirc \rrbracket_{v_{+}}^{r} \oplus$ $\llbracket \Omega \rrbracket^{r-1}$ is such that

$$
\left(\begin{array}{cc}
d_{0}^{r} & 0 \\
m & -d_{1}^{r-1}
\end{array}\right)\left(\begin{array}{l}
a \\
b
\end{array}\right)=\left(\begin{array}{c}
d_{0}^{r} a \\
\breve{m} a-d_{1}^{r-1} b
\end{array}\right)=\left(\begin{array}{l}
0 \\
0
\end{array}\right)
$$

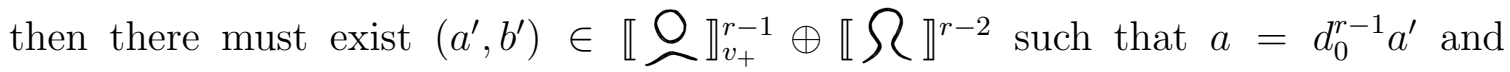
$b=\breve{m}^{r-1} a^{\prime}-d_{1}^{r-2} b^{\prime}$.

First, we claim that $\breve{m}^{*}$ is a bijection and begin by showing $\breve{m}$ is surjective. Every smoothing of $\Omega$ has a corresponding smoothing of $\bigcirc$, which is the same smoothing and only differs inside our chosen crossing. Therefore, take an element $x$ of $V_{\alpha} \subset \llbracket \Omega \rrbracket^{r}$. The element $x$ has an element of $V$ corresponding to each cycle. Hence, if we consider the corresponding $V_{\alpha} \subset \mathbb{U} \Omega \rrbracket^{r}$, the element with the same element of $V$ assigned to each cycle and $v_{+}$assigned to the extra cycle will be in the preimage of $x$.

More explicitly, take $x \otimes x^{\prime} \in V_{\alpha} \subset \mathbb{U} \Omega \rrbracket^{r}$, where $x$ is the element of $V$ representing the cycle in $\Omega$, and $x^{\prime}$ represents the rest of the cycles in the rest of the smoothing. Then $m \otimes \operatorname{id}\left(v_{+} \otimes x \otimes x^{\prime}\right)=x \otimes x^{\prime}$, since $m$ is the identity on tensor products with $v_{+}$, that is $v_{+} \otimes\left(r \cdot v_{+}\right) \stackrel{m}{\longmapsto} r \cdot v_{+}$and $v_{+} \otimes\left(r \cdot v_{-}\right) \stackrel{m}{\longmapsto} r \cdot v_{-}$for every $r \in R$. This is true for every generator $V_{\alpha} \subset \mathbb{I} \Omega \rrbracket^{r}$. So in fact, we have shown that there is an element in the preimage that is also an element of $\llbracket \bigcirc \rrbracket_{v_{+}}^{n-1}$, as required.

Now for the injectivity of $\breve{m}$. If $m \otimes \operatorname{id}\left(v_{+} \otimes x \otimes x^{\prime}\right)=m \otimes \operatorname{id}\left(v_{+} \otimes y \otimes y^{\prime}\right)$, then $x \otimes x^{\prime}=y \otimes y^{\prime}$. Clearly, it follows that $\breve{m}$ is an injection.

Now we are convinced that $\breve{m}^{r-1}: \llbracket \cong \rrbracket_{v_{+}}^{r-1} \rightarrow \llbracket \Omega \rrbracket^{r-1}$ is a bijection, we can 


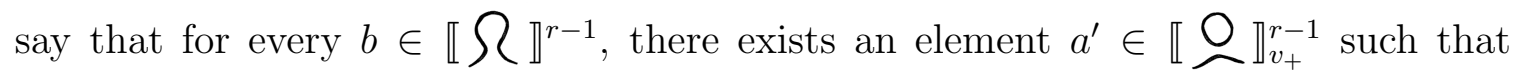
$\breve{m}^{r-1}\left(a^{\prime}\right)=b$. Therefore, since $(a, b)$ is a cocycle and $\breve{m}^{*}$ is a chain map, $\breve{m} a-$ $\breve{m} d_{0}^{r-1} a^{\prime}=\breve{m} a-d_{1}^{r-1} \breve{m} a^{\prime}=0$. As $\breve{m}^{r}$ is injective, and $\breve{m}\left(a-d_{0}^{r-1} a^{\prime}\right)=0, a=d_{0}^{r-1} a^{\prime}$. So, for $\left(a^{\prime}, 0\right) \in \llbracket \stackrel{\bigcirc}{\bigcirc} \rrbracket_{v_{+}}^{r-1} \oplus \mathbb{\|} \Omega \rrbracket^{r-2}$, we have that $d\left(\left(a^{\prime}, 0\right)\right)=\left(d_{0}^{r-1} a^{\prime}, \breve{m} a^{\prime}\right)=(a, b)$, so $(a, b)$ is a coboundary, as required.

Note that though $\breve{m}^{*}$ is a bijection, it is not an isomorphism of graded modules, since it has a $q$-shift of -1 . That is, if $\operatorname{deg}(x)=i$ for $x \in \llbracket \cong \rrbracket^{r}$, then $\operatorname{deg}\left(\breve{m}^{r}(x)\right)=i-1$.

Now we know the subcomplex $\llbracket \bigcirc \rrbracket_{v_{+}}^{*} \oplus \llbracket \Omega \rrbracket^{*-1}$ is acyclic, we apply the cancellation theorems:

$$
\begin{aligned}
& H^{r}\left(\llbracket \bigcirc \rrbracket^{*} \oplus \llbracket \Omega \rrbracket^{*-1}\right) \cong H^{r}\left(\left(\llbracket \bigcirc \rrbracket^{*} \oplus \llbracket \Omega \rrbracket^{*-1}\right) /\left(\llbracket \bigcirc \rrbracket_{v_{+}}^{*} \oplus \llbracket \Omega \rrbracket^{*-1}\right)\right)
\end{aligned}
$$

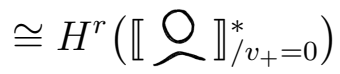

for all $n$. It is clear that in the second isomorphism, all elements of the form $v_{+} \otimes x$ are equivalent to 0 . Therefore, all elements are generated by elements of the form $v_{-} \otimes x$.

It remains to show that $H^{r}\left(\llbracket \bigcirc \rrbracket_{/ v_{+}=0}^{*}\right)$ is isomorphic to $H^{r}\left(\llbracket \Omega \rrbracket^{*}\{-1\}\right)$ for all $n$. It easily follows that the homologies after the shift by $\cdot\left[-n_{-}\right]\left\{n_{+}-2 n_{-}\right\}$will also be isomorphic, and in both cases, this will give the Khovanov chain complex of the respective oriented knots, as $\Omega$ has one less positive crossing than 2 , which is adjusted for in the $q$-shift $\cdot\{-1\}$.

Consider the element $x \otimes v_{-}$in $\llbracket \bigcup_{v_{v_{+}=0}^{r}}^{r}$, and denote its $q$-degree by $k \in \mathbb{Z}$. Consider the isomorphism of vector spaces $x \otimes v_{-} \mapsto x$. This is a bijection, but to show it is an isomorphism it remains to show that this does not change the $q$-degree of the element. If $\operatorname{deg}\left(x \otimes v_{-}\right)=k$, then $\operatorname{deg}(x)=k+1$, but because of the $q$-shift $\cdot\{-1\}$ which shifts everything in the graded module "down" by 1 , the $q$-dimension of $x \in \llbracket \Omega \rrbracket^{*}\{-1\}$ is altered by -1 (see Definition 3.3.10). Thus, the shift of $x$ is 
adjusted accordingly, since $\operatorname{deg}(x)=k+1-1=k$, as required.

The differentials are identical in $\llbracket \bigcirc \rrbracket_{v_{-}}^{*}$ and $\llbracket \Omega \rrbracket^{*}\{-1\}$, since the two knot projections are identical outside this section and all differentials are unaffected by the extra cycle in $\bigcirc$. This means that the $n$-dimensional cubes are identical except for an extra cycle generated by $v_{-}$in $\llbracket \rrbracket_{v_{-}}^{*}$, which is unaffected by the differential.

This completes the proof that the Khovanov homology of a link is invariant under R1.

\subsection{Second Reidemeister move}

Let $D$ denote the knot projection with crossings as follows, $\mathcal{Y}$, and $D^{\prime}$ denote the same knot projection with the previous crossing replaced by $\sim$. We denote the projections of $D$ with the crossings in $\gamma$ replaced by smoothings of $D$ with subscripts representing the smoothings. Without loss of generality, let the left crossing in $\mathcal{Y}$ be the first crossing of $D$, and let the right crossing be the second crossing of $D$. In other words, $D_{00}$ denotes $) \simeq, D_{01}$ denotes $) \bigcirc\left(, D_{10}\right.$ denotes $\approx$, and $D_{11}$ denotes $\cong$ (.

We will discontinue the use of $\breve{m}$ for the map $m \otimes$ id, and use $m$ instead, where the tensor product with the identity is implicit.

Proof of invariance of the Khovanov homology under $\boldsymbol{R} 2$. We start by introducing a new way to write the cochain complex graded vector space $\llbracket D \rrbracket$ as a diagram. Let $\alpha$ be a smoothing that resolves every crossing in $D$ except the first and second crossings which are seen in $\mathcal{Y}$. Then if $|\alpha|=r$, then the following chain complex represents 
all the smoothings of $D$ which contain $\alpha$. These are $00 \alpha, 01 \alpha, 10 \alpha$, and $11 \alpha$.

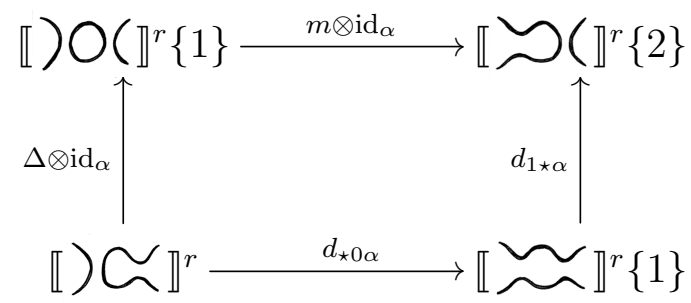

$\llbracket D \rrbracket$

This diagrams holds for every $\alpha \in\{0,1\}^{n-2}$. Recall from the proof of $\mathbf{R} 1$ that the maps pictured above will form chain maps on the brackets of $D_{00}, D_{01}, D_{10}$, and $D_{11}$. As in the discussion at the beginning of this chapter, what we have drawn in this diagram is chain complex maps between Khovanov brackets, and these maps are "pre-flattened," hence all produce commuting ladder diagrams. Nonetheless, we keep in mind that we are implicitly taking direct sums and including some minuses on the differentials.

It will be satisfactory to show that the following cochain complexes are quasi-isomorphic. These diagrams above account for the normalisation $\left[-n_{-}\right]\left\{n_{+}-2 n_{-}\right\}$, since no matter how one orients the strands, $\mathcal{Y}$ has exactly one more negative crossing and one more positive crossing than $\sim$. Therefore, we know the shift of $D$ will be $\left[-n_{-}\right]\left\{n_{+}-2 n_{-}\right\}$, so the shift of $D^{\prime}$ will be $\left[-\left(n_{-}-1\right)\right]\left\{\left(n_{+}-1\right)-2\left(n_{-}-1\right)\right\}$ which is the same as $\left[-n_{-}\right]\left\{n_{+}-2 n_{-}\right\}[1]\{1\}$. 


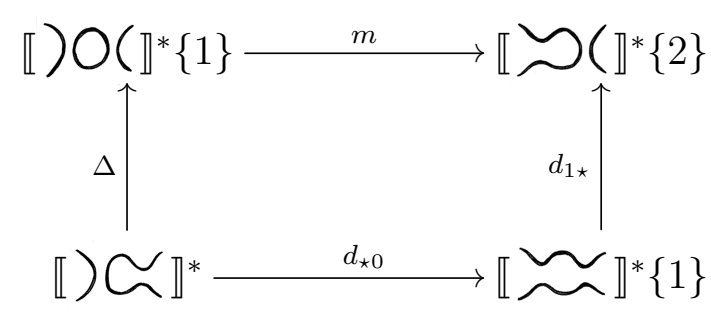

$\llbracket D \rrbracket^{*}$

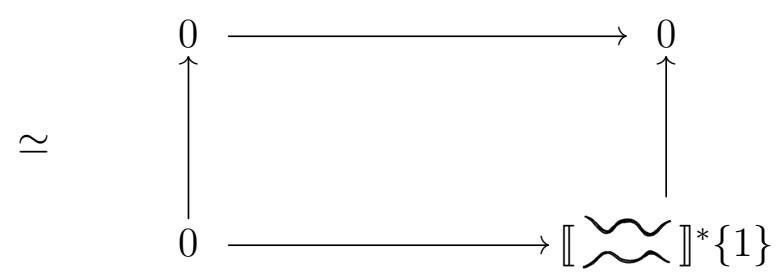

$\llbracket D^{\prime} \rrbracket^{*}[1]\{1\}$

As mentioned previously, these are cochain complex maps in the same way as $m$ : $\bigcirc \rightarrow \Omega\{1\}$ was in the first Reidemeister move. The chain complex on the left represents the bi-complex

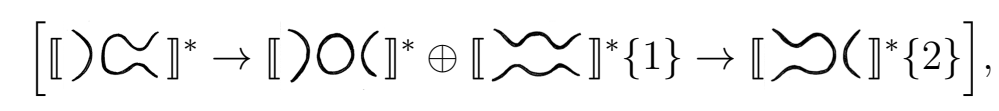

where the first map is represented by $\left(\begin{array}{c}\Delta \\ d_{\star 0}\end{array}\right)$, and the second map is represented by $\left(\begin{array}{ll}m & d_{1 \star}\end{array}\right)$. In other words, the modules of the chain complex are the direct sums of the downward right diagonals, with differentials the sum of the maps of the direct summands. One can regard the number of arrows, $s$, from the bottom left to one of the vertices in this diagram as a visual way to represent homological degree shifts, or $[s]$, at this vertex.

We remark that it is less convenient to use the cone construction here as we would end up with a $4 \times 4$-matrix. Nonetheless, what remains of the construction built in R1 is the method of the chain maps as matrices. Also what remains is the validity of taking quotients of the chain complexes when drawn as squares. Recall it is equivalent to rewrite the above as $\llbracket \mathcal{Q} \rrbracket^{*} \simeq \llbracket \widetilde{\sim} \rrbracket^{*-1}\{1\}$. For clarity, the asterisk superscripts $*$, and occasionally the shifts $\{\cdot\}$ will be omitted in these diagrams, as above. 
Consider the following subcomplex of $\llbracket \mathcal{\gamma} \rrbracket^{*}$.

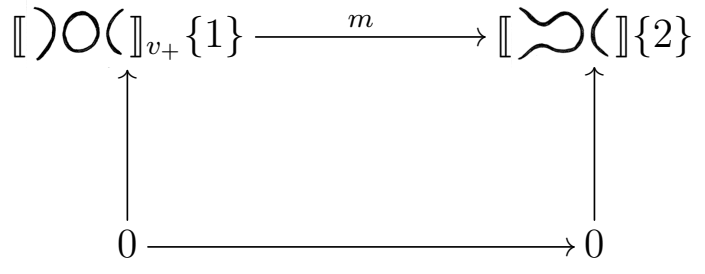

$\mathcal{C}^{\prime}$

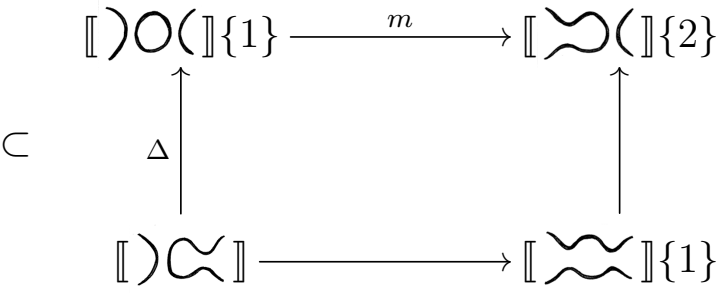

$\mathcal{C}$

Conveniently, we have shown that the complex $\mathcal{C}^{\prime}$ on the left (it is identical up to the shift $\cdot[1])$ is acyclic in the proof of $\mathbf{R} \mathbf{1}$, and so by the cancellation theorems, $\mathcal{C} \simeq \mathcal{C} / \mathcal{C}^{\prime}$.

The quotient $\mathcal{C} / \mathcal{C}^{\prime}$ is as follows.

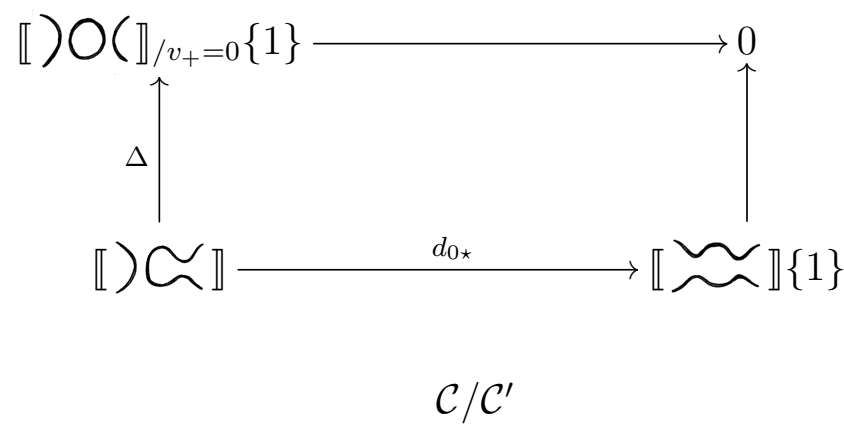

Next, one finds another acyclic subcomplex of $\mathcal{C} / \mathcal{C}^{\prime}$, call it $\mathcal{C}^{\prime \prime}$.

Without loss of generality, let the first element of the tensor product represents the cycle in $) O($, and the second element represents the rest of the cycles. We show that $\Delta$ in $\mathcal{C} / \mathcal{C}^{\prime}$ is a bijective map. If $v_{+}$is assigned to the right cycle in $D_{00}$, $\Delta \otimes \operatorname{id}\left(v_{+} \otimes x\right)=\left(v_{+} \otimes v_{-}+v_{-} \otimes v_{+}\right) \otimes x \sim v_{-} \otimes v_{+} \otimes x$, since $v_{+} \otimes v_{-} \otimes x=0$ in this quotient of the graded vector space $\llbracket) O\left(\rrbracket_{/ v_{+}=0}^{r}\right.$ for every $x$. So every ele- 
ment of $\llbracket D_{00} \rrbracket$ is sent to the same element but with $v_{-}$assigned to the cycle inside $D_{01}$.

As the map $\Delta$ is bijective, one can define a map $\tau:=d_{0 \star} \Delta^{-1}$. We write elements

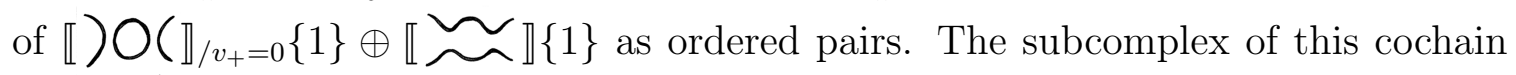
complex is the collection of elements of the form $(\beta, \tau \beta)$, where $\beta$ is any element of $\llbracket) \bigcirc\left(\rrbracket_{/ v_{+}=0}^{r}\{1\}\right.$ for some $r$.

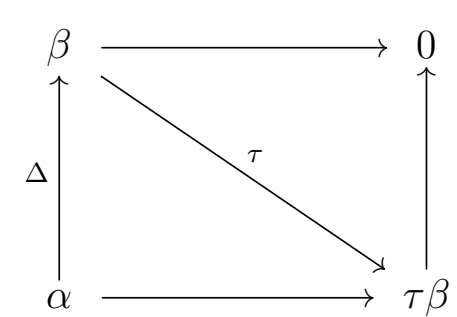

$\mathcal{C}^{\prime \prime}$

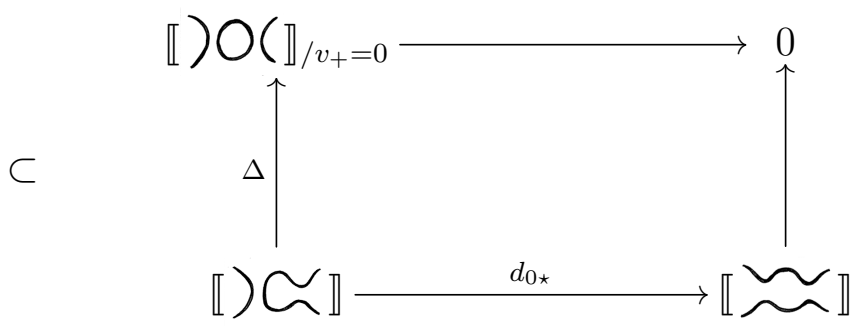

$\mathcal{C} / \mathcal{C}^{\prime}$

Note that above we have written the generating elements of the complex rather than the complex itself. The complex can also be written as follows.

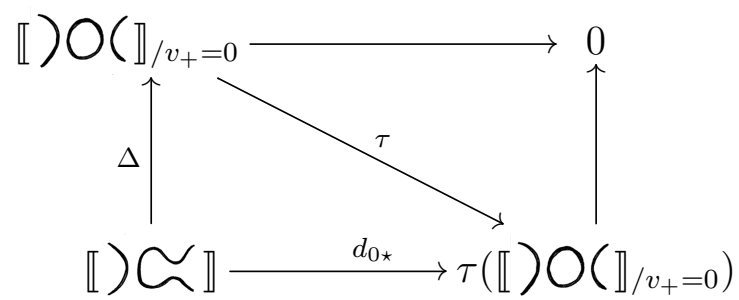

$\mathcal{C}^{\prime \prime}$

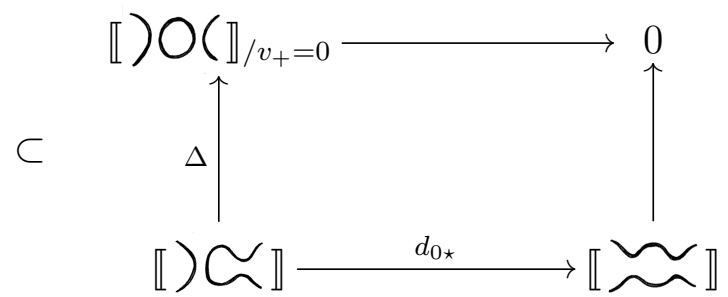

$\mathcal{C} / \mathcal{C}^{\prime}$

We claim that $\mathcal{C}^{\prime \prime}$ is acyclic. The chain map is well defined, since $d_{0 \star}(\alpha)=\tau \Delta(\alpha)$, so $\left(\Delta \alpha, d_{0 \star}(\alpha)\right)=(\Delta \alpha, \tau(\Delta \alpha))$, which is an element in the complex $\mathcal{C}^{\prime \prime}$. The assignment $\alpha \mapsto\left(\Delta \alpha, d_{0 \star} \alpha\right)$ is a bijection, since $\Delta: \llbracket D_{00} \rrbracket^{*} \rightarrow \llbracket D_{01} \rrbracket_{/ v_{+}=0}^{*}\{1\}$ itself is.

Now one mods out by $\mathcal{C}^{\prime \prime}$, so all elements of the complex $\mathcal{C}^{\prime \prime}$ go to zero. There- 
fore, $(\beta, \tau \beta)=0$ for every $\beta \in \llbracket D_{01} \rrbracket / v_{+}=0$ in this quotient complex. In other words, the quotient imposes the equivalence $(\beta, 0) \sim(0,-\tau \beta)$ for every $\beta$. We will call this complex $\mathcal{C}_{\tau}$.

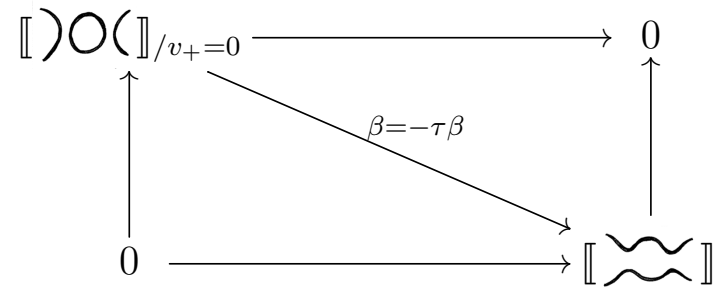

$$
\mathcal{C}_{\tau}:=\left(\mathcal{C} / \mathcal{C}^{\prime}\right) / \mathcal{C}^{\prime \prime}
$$

generated by

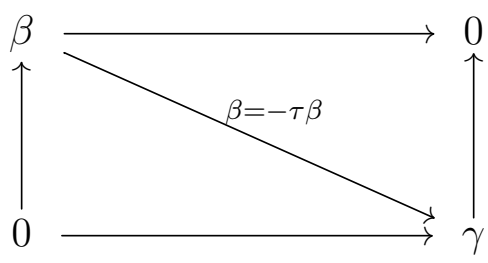

$\mathcal{C}_{\tau}:=\left(\mathcal{C} / \mathcal{C}^{\prime}\right) / \mathcal{C}^{\prime \prime}$

When discussing this complex we will always use $\beta$ to denote an element of $\llbracket) \bigcirc\left(\rrbracket_{/ v_{+}=0}^{r}\right.$, and $\gamma$ to denote an element of $\llbracket \sim \rrbracket^{r}$, as above, which shows two different ways to write the same chain complex.

We claim the elements of $\llbracket \sim \rrbracket$ can be (naturally) embedded into this quotient. Consider the inclusion $\iota: \llbracket \widetilde{\sim} \mathbb{1}^{r} \hookrightarrow \mathcal{C}_{\tau}^{r}$ which maps $\gamma \mapsto(0, \gamma)$. Take $\gamma_{1}, \gamma_{2} \in$ $\llbracket \approx \rrbracket^{r}$ such that $\iota\left(\gamma_{1}\right)=\iota\left(\gamma_{2}\right)$. Then $\left(0, \gamma_{1}-\gamma_{2}\right)=(\beta, \tau \beta)$ for some $\beta$, and clearly $\beta=0$, so $\gamma_{1}-\gamma_{2}=\tau \beta=0$, and are the same element of $\llbracket \sim \rrbracket^{r}$.
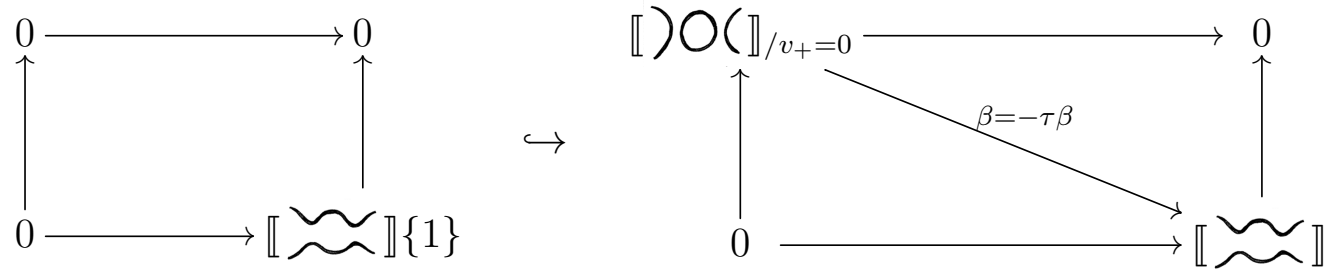

$$
\mathcal{C}_{\tau}:=\left(\mathcal{C} / \mathcal{C}^{\prime}\right) / \mathcal{C}^{\prime \prime}
$$

We have shown the above is an embedding. Nonetheless, it remains to show that this embedding is an isomorphism. Let $[\cdot]_{\sim}$ denote the equivalence classes of elements under this quotient. We want to show that this embedding is also surjective, and we 
only need to show that every element $(\beta, \gamma)$ is equivalent to $\left(0, \gamma^{\prime}\right)$ for some $\gamma^{\prime}$. This follows if we choose $\gamma^{\prime}=\gamma-\tau \beta$, then $(\beta, \gamma)-(0, \gamma-\tau \beta)=(\beta, \tau \beta)$, which is an element of $\{(\beta, \tau \beta): \beta \in \mathbb{}) \bigcirc\left(\rrbracket^{r}\right\}$, as required.

This shows the invariance under $\mathbf{R} \mathbf{2}$, as required.

\subsection{Third Reidemeister move}

Proof of invariance of the Khovanov homology under R3. Consider an oriented knot projection $D$ with a section of the projection which looks like knot projection but with

These projections will be denoted simply by respectively. For the third Reidemeister move, we want to show that the Khovanov bracket of these two projections are quasi-isomorphic.

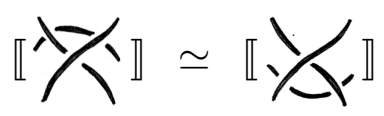

This is sufficient - that is we don't need to consider any shifts - since no matter how one orients the strands in the projections (that are consistent with the surrounding knot), the two knots will have the same number of positive crossings and negative crossings. Therefore, both (oriented) knot projections have the shift $\left[-n_{-}\right]\left\{n_{+}-2 n_{-}\right\}$.

As before, we now smooth the crossings pictured, which are ordered as follows. Where required, the subscript ' $a$ ' will denote when something occurs on the left knot ( ), and ' $b$ ' will denote when something occurs on the right knot ( 

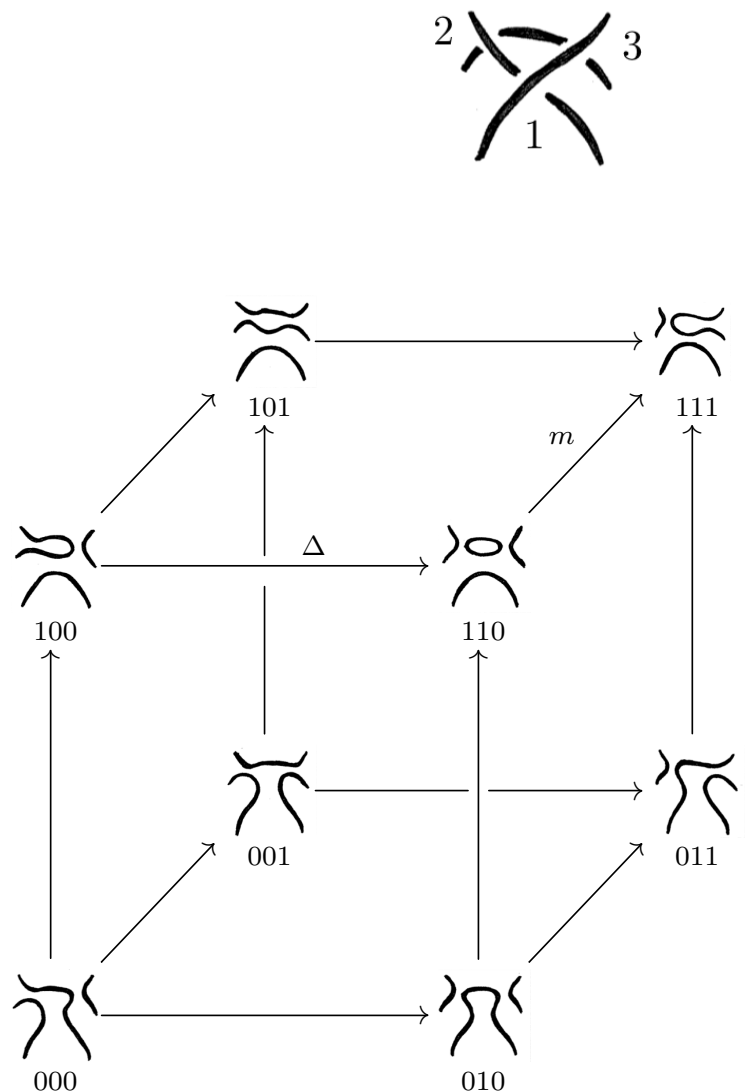
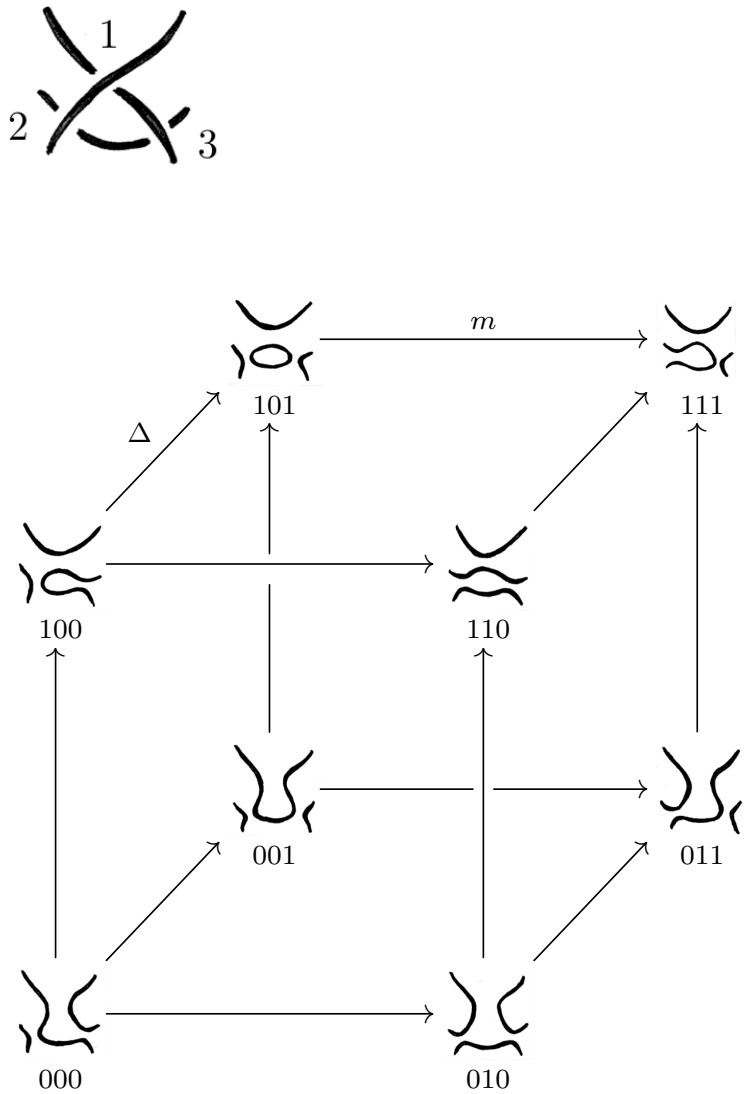

Note, we haven't included the $q$-shifts $\{\cdot\}$, and brackets $\llbracket \cdot \rrbracket$, which we will continue to omit along with the smoothing label. Another way to think of the above is as the chain complexes of the following, where only the first crossing has been replaced by a smoothing.
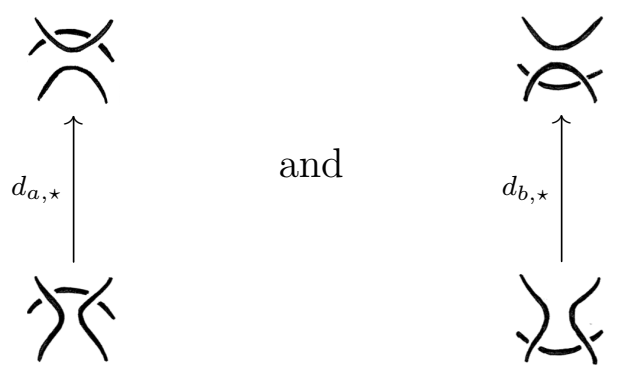

Next, one replaces the top layer of the cube using the complex $\mathcal{C}_{\tau}$ from the proof of $\mathbf{R 2}$ in the previous section. That is, the top layer is quasi-isomorphic to the following quotient chain complex. 


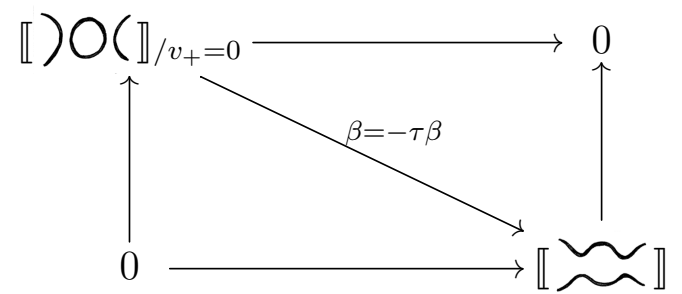

More rigorously, we find the same sub-complexes and take the same quotients of the above cubes by the complex $\left[0 \rightarrow \mathcal{C}^{\prime}\right]$, and so on.
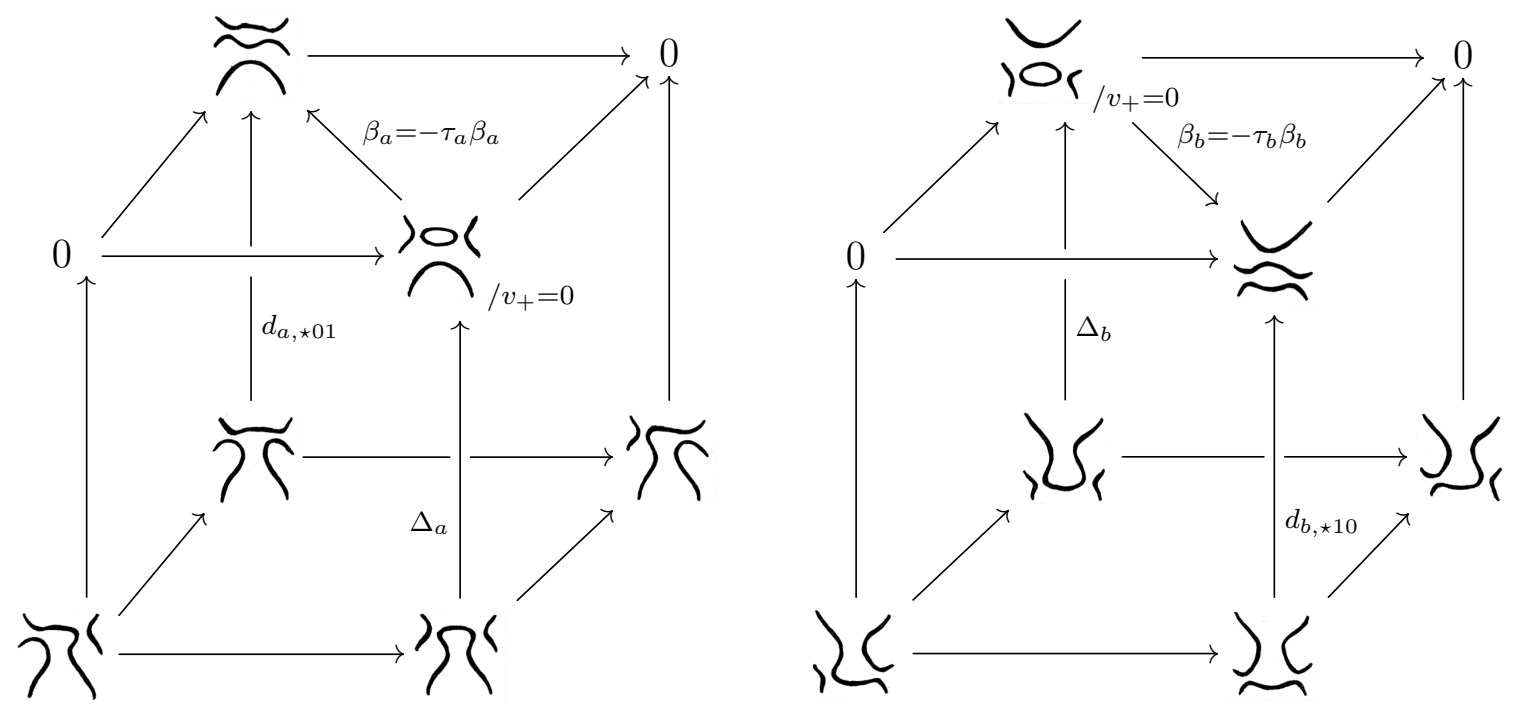

Now, we would like to construct an isomorphism between these two chain complexes. The isomorphism between the bottom layers is clear, and the isomorphism of the top layers is just a transpose of the direct sums. Recall, the top layers are isomorphic since they are both isomorphic to $\asymp$. For this isomorphism on spaces - let us call it $\Upsilon$ - to also be an isomorphism of chain complexes, the only thing that remains to show is that the vertical maps between the layers commute with the isomorphism $\Upsilon$ between the cubes.

Let $\Upsilon$ act on the top layers by sending $\left[\left(0, \gamma_{a}\right)\right] \mapsto\left[\left(0, \gamma_{b}\right)\right]$. To show that this isomor- 
phism on spaces commutes with the differentials, one must prove the following.

$$
d_{a, \star 01}=\tau_{b} \Delta_{b} \quad \text { and } \quad d_{b, \star 10}=\tau_{a} \Delta_{a}
$$

where $d_{a, \star 10}=\Delta_{a}$ and $d_{b \star 01}=\Delta_{b}$. We want to show because, say $\Upsilon$ is the isomorphism, then we require $\Upsilon d_{a, \star 01}=\Delta_{b} \Upsilon$ but

$$
\operatorname{im}\left(\Upsilon d_{a, \star 01}\right) \subseteq \mho_{/ i m} \tau_{b} \quad \text { and } \quad \operatorname{im}\left(\Delta_{b} \Upsilon\right) \subseteq \bigcup_{\mathbf{S}_{/ v_{+}=0}}
$$

So, we must consider what the images are under the equivalence relation $\beta \sim \tau_{b} \tau$. If $\Upsilon d_{a, \star 01}=-\tau_{b} \Delta_{b} \Upsilon$, then these maps are equal since $(\beta, 0) \sim(0,-\tau \beta)$ under the equivalence relation. If one ignores the $\Upsilon$ - which one can since it is the identity on the spaces it is acting on in this case - the equation is exactly that in (5.1). It is similar for the second.

Now the task is to show that these two relations are equivalent. Recall that, using the notation of the diagrams, that $\tau_{a}:=d_{a, 10 \star} \Delta^{-1}$ where $\Delta$ is the map $d_{a, 1 \star 0}$. We denote the map $\Delta_{a}$ which is mapped to the space $\stackrel{>}{\wedge} / v_{+}=0$ by $\overline{\Delta_{a}}$. (This just means $\overline{\Delta_{a}}=\phi \circ \Delta_{a}$, where $\phi$ mods out by $\left.v_{+}\right)$.

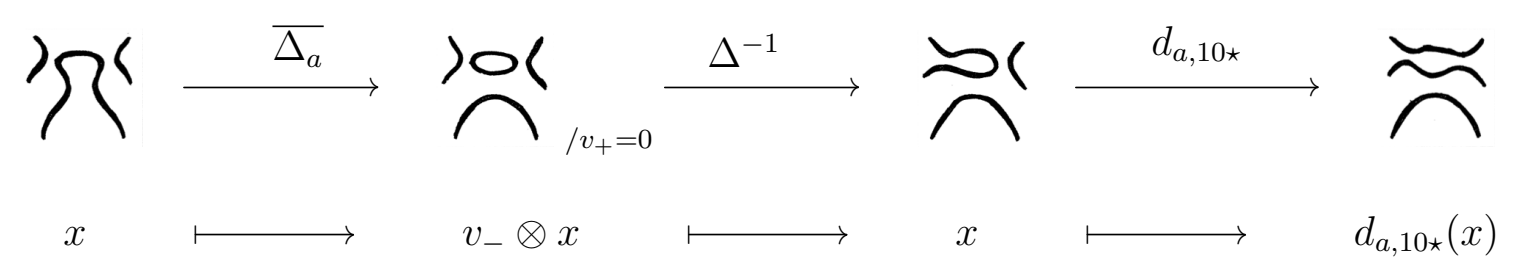

Recall $\Delta^{-1}$ from $\mathbf{R 2}$. It is clear from the above maps that $\Delta^{-1} \overline{\Delta_{a}}=$ id. Therefore $\tau_{a} \Delta_{a}=d_{a, 10 \star}$. Note also that the edges of the cube,

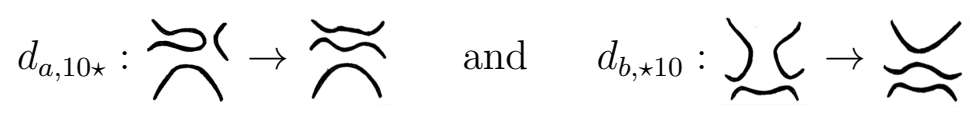


are the same rearrangement of the top two strands, and so are the same map. Given the morphism $\Upsilon$ is the identity on the bottom layer of the cube, $\Upsilon$ commutes with this side of the cube, as in equation 5.1 .

As for the proof of $d_{a, \star 01}=\tau_{b} \Delta_{b}$, it is almost identical to the one given above. Recall that

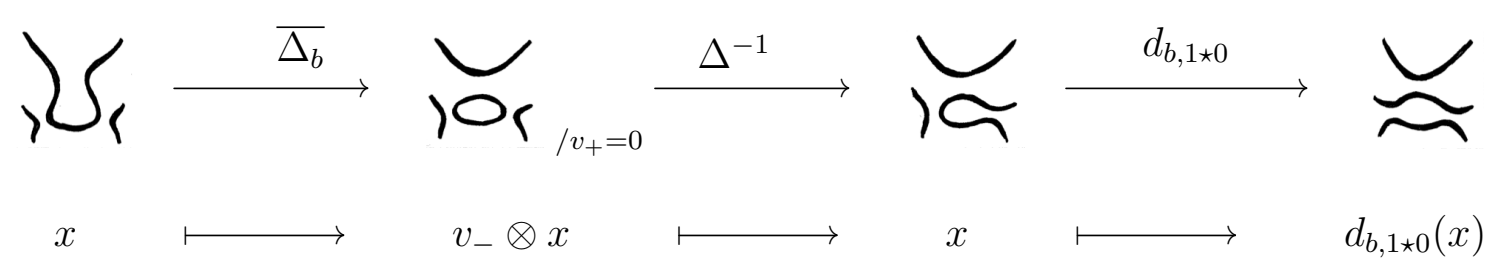

which implies that $\Delta^{-1} \overline{\Delta_{b}}=\mathrm{id}$, and so $\tau_{b} \Delta_{b}=d_{b, 1 \star 0}$. Accordingly, we have

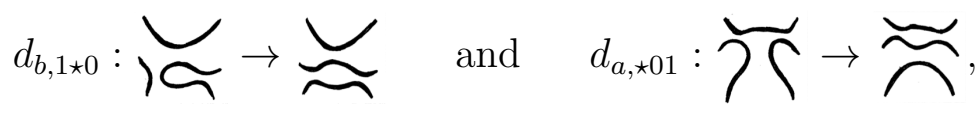

are the same, as required.

Therefore, the Khovanov homology is invariant under R3, as required.

In Dror Bar-Natan's proofs in [1], we see that these proofs are (as you would expect) not an algebraic analogue of the proofs of Reidemeister invariance of the Jones polynomial.

This is not apparent in the proof of $\mathbf{R} \mathbf{1}$, but, as shown explicitly by Bar-Natan, we see that it is not possible to use $\mathbf{R} \mathbf{2}$ in the invariance of $\mathbf{R 3}$. Specifically, though we

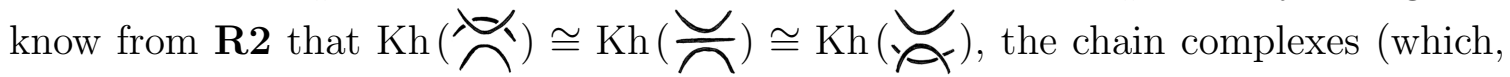
recall, are isomorphic on each space), 

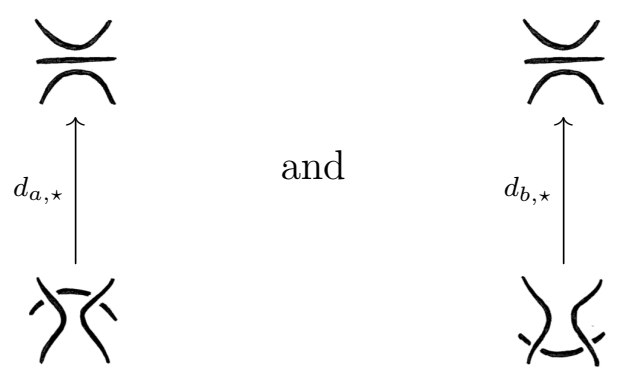

cease to be isomorphic as chain complexes.

Instead, by using the quotient complex isomorphic to of the chain complexes that also commutes with the differentials $d_{a, \star}$ and $d_{b, \star}$. 


\section{Chapter 6}

\section{Topological Quantum Field Theories and Frobenius objects}

The purpose of this chapter is to show, from the bottom up, an equivalence of categories between $(1+1)$-TQFTs and Frobenius algebras. This is useful because the graded $R$-module $V$ in Chapter 4 is a Frobenius object in the category of $\mathbb{Z}$-graded $R$-modules, which we will show in Chapter 7. Hence, one can apply the TQFT to replace all algebraic relations with something more topological. This simplifies much of the working. In Chapter 7 we also use TQFTs to review some aspects of the Khovanov homology.

In sections 6.1 and 6.2, we aim to describe an isomorphism of categories,

$$
\operatorname{SymMonCat}(X, V) \cong \operatorname{cFrob}(\mathrm{V})
$$

between the symmetric monoidal functor category and Frobenius objects in the category $\mathbf{V}$. This involves the construction of the free monoidal category $\mathbf{X}$, with desired relations. In Section 6.3, we discuss, as promised, a topological description of a category of cobordisms. We see in this section the geometric reasoning the construction of the category $\mathbf{X}$, as all relations reflect the topology of surfaces. Also, we show the above isomorphism of categories in the context of 2-cobordisms in Section 6.5. This all follows [7], except with a rearrangement of the material, by instead building up 
the isomorphism from a category-theoretic point of view. Lastly, in this chapter we mention the symmetric monoidal category we consider for applying this chapter to the Khovanov homology in the final chapter of this thesis.

\section{Some category theory notation}

For a category $\mathcal{C}$, let $\operatorname{Ob}(\mathcal{C})$ denote the collection of objects of $\mathcal{C}$, and let $\operatorname{Mor}(\mathcal{C})$ denote the collection of morphisms of $\mathcal{C}$. An equivalence of categories is denoted $\simeq$, and an isomorphism of categories is denoted $\cong$. We assume the reader is familiar with the basics of category theory. Our source for this material is [10].

\subsection{Symmetric monoidal categories}

\section{Symmetric monoidal categories}

\section{Definition 6.1.1. Strict monoidal category}

Let $\{*\}$ be the category with exactly one object, $*$, and one morphism, $\mathrm{id}_{*}$. A strict monoidal category is a category $\mathbf{V}$ with a bi-functor $m$ and functor $h$,

$$
m: \mathbf{V} \times \mathbf{V} \rightarrow \mathbf{V} \quad h:\{*\} \rightarrow \mathbf{V}
$$

which satisfy

$$
m \circ\left(m \times \operatorname{id}_{\mathbf{V}}\right)=m \circ\left(\operatorname{id}_{\mathbf{V}} \times m\right), \quad m \circ\left(h \times \operatorname{id}_{\mathbf{V}}\right)=\operatorname{id}_{\mathbf{V}}=m \circ\left(\operatorname{id}_{\mathbf{V}} \times h\right) .
$$

That is, the following diagrams commute.

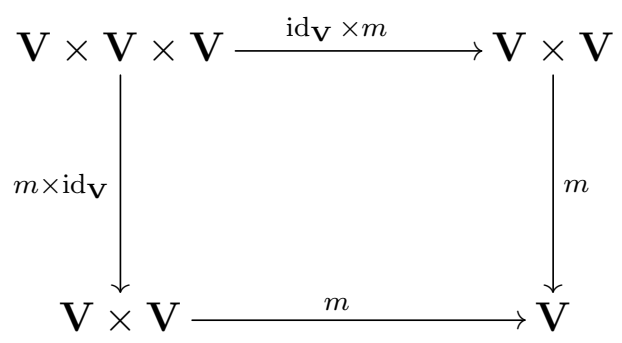



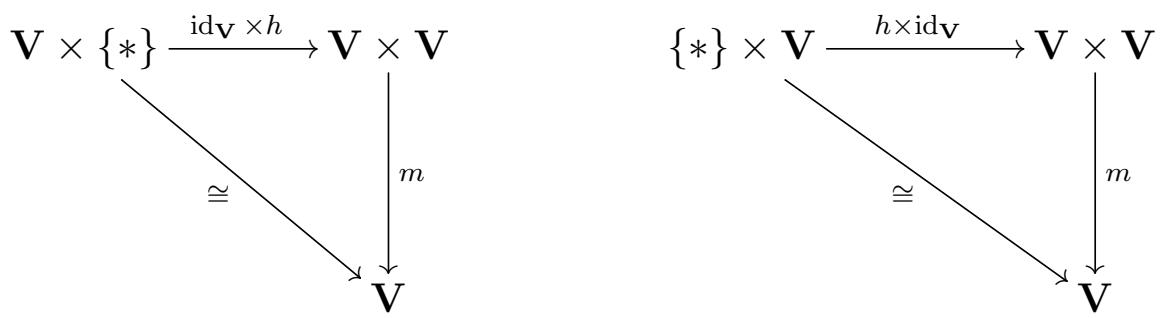

The isomorphisms $\mathbf{V} \times\{*\} \cong \mathbf{V} \cong\{*\} \times \mathbf{V}$ are the projection morphisms onto $\mathbf{V}$, which are isomorphisms of categories.

It is convenient to define an object $I:=h(*)$, and to define an object $X \square Y:=$ $m(X, Y)$ for every $X, Y \in \mathrm{Ob}(\mathbf{V})$, and similarly for $f, g \in \operatorname{Mor}(\mathbf{V})$, the morphism $f \square g:=m(f, g)$. The top diagram implies associativity of $\square,(X \square Y) \square Z=$ $X \square(Y \square Z)$. The bottom diagrams imply there is an identity object $h(*)$, denoted $I$, that commutes with every object $X, X \square I=X=I \square X$.

The bi-functor $m$ is referred to as the monoidal product and $I$ is referred to as the neutral object in $\mathbf{V}$. A monoidal category will be denoted by a triple $(\mathbf{V}, \square, I)$, with respect to its monoidal product and neutral object.

\section{A coherence theorem}

The word strict in strict monoidal category refers to the equalities $X \square I=X=I \square X$ and $(X \square Y) \square Z=X \square(Y \square Z)$. In a monoidal category, these equalities are only required to be isomorphisms, and we will formally define these in the next paragraphs. Then, we will give a brief overview of why it is sufficient to only discuss the strict case, in the method of [10]. 
The following morphism is natural for all $a, b, c \in \mathrm{ObV}$, and the subsequent pentagonal diagram commutes for all $W, X, Y, Z \in \mathrm{ObV}$.

$$
\alpha=\alpha_{X, Y, Z}: X \square(Y \square Z) \cong(X \square Y) \square Z
$$

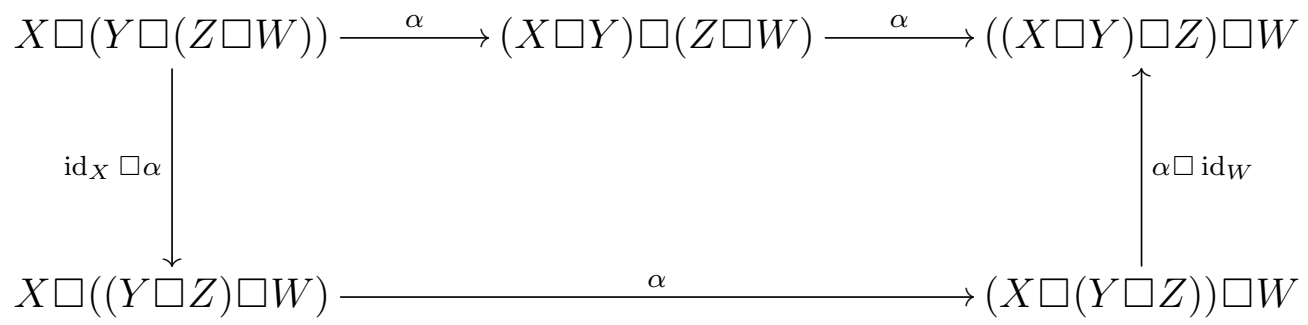

This shows associativity of the monoidal product up to natural isomorphism. Next, we have the following natural isomorphisms for all $X \in \mathrm{ObV}$.

$$
\lambda_{X}: I \square X \cong X, \quad \rho_{X}: X \square I \cong X .
$$

Such that

$$
\lambda_{I}=\rho_{I}: I \square I \rightarrow I,
$$

and the following three diagrams commute.
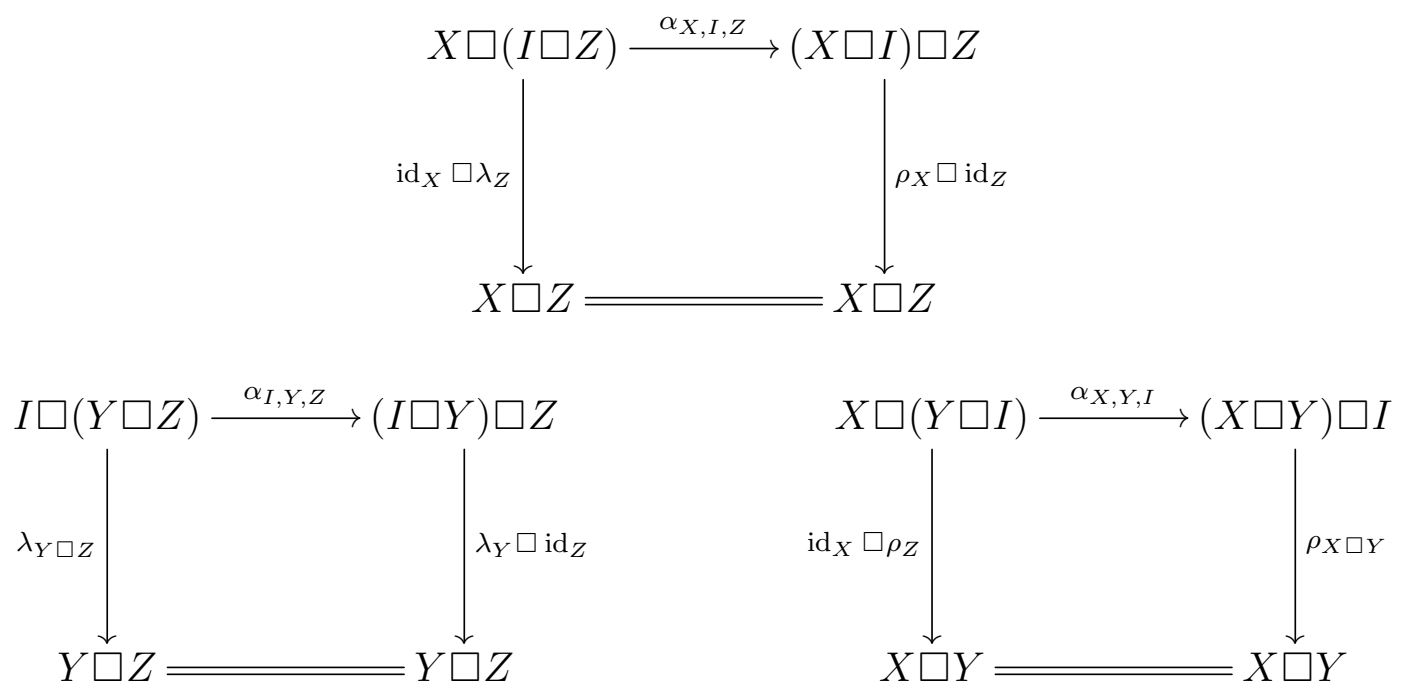
The categories we want to look at will not always be strict monoidal categories, but it will be convenient in the following section to work with only strict monoidal categories. We can do this as discussed in Mac Lane, [10] in Sections VII and XI, which give us the following theorems.

First, Mac Lane proves a theorem that gives an isomorphism between any two strings of objects of $\mathbf{V}$. That is, consider an $n$-tuple of $\mathbf{V}$, or an element of $\mathrm{Ob}\left(\mathbf{V}^{n}\right)$, and let $w, v$ bring each ordered element to a monoidal product combinations of brackets and neutral elements $I$ of the ordered string. For example, consider $w$ which assigns $\left(X_{1} \square I\right) \square X_{2}$ to $\left(X_{1}, X_{2}\right)$ (the ordering of the $X_{i}$ must stay the same). Mac Lane proves that there exists a natural isomorphism between any two elements of this form. The following theorem is a similar assertion of the previous statement.

\section{Theorem 6.1.2. Coherence Theorem}

Every monoidal category is categorically equivalent to a strict monoidal category.

This theorem allows us to say any commuting diagram of a monoidal category is equivalent to one in a strict monoidal category. As a result, in this section we will only consider strict monoidal categories from this point on, and bear in mind that everything can be generalised to general monoidal categories.

\section{"Stick figure" notation of morphisms}

It is useful to visualise objects of a monoidal category as vertical stacks of dots, where the $i$ th dot from the bottom represents $X_{i}$ in $X_{1} \square X_{2} \square \cdots \square X_{n}$.

$$
\begin{array}{cc} 
& X_{n} \\
X_{1} \square X_{2} \square \cdots \square X_{n} \text { is represented by } \quad X_{3} \\
X_{2} \\
X_{1}
\end{array}
$$


Morphisms are represented by lines, with the domain the left dot and the co-domain the right dot of the line. The morphism $f: X \rightarrow Y$ will be the following.

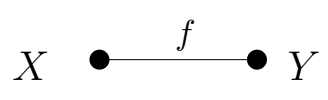

Additionally, it will be useful later to use the following property of monoidal categories. Suppose $f: X \rightarrow X^{\prime}$ and $g: Y \rightarrow Y^{\prime}$ are morphisms in $\mathbf{V}$. Then the following are equivalent.

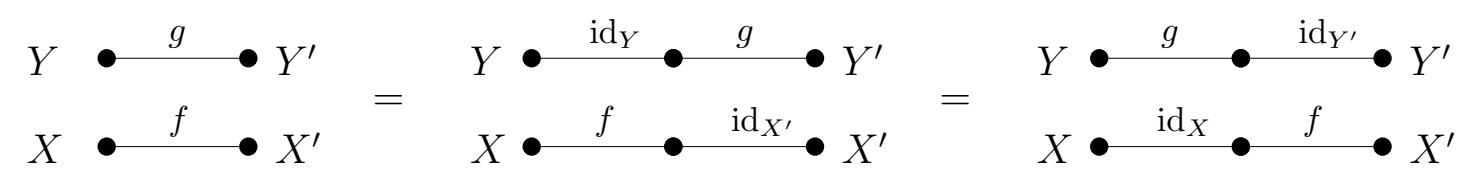

More specifically, this means $f \square g=\left(\operatorname{id}_{X^{\prime}} \square g\right) \circ\left(f \square \operatorname{id}_{Y}\right)=\left(f \square \operatorname{id}_{Y^{\prime}}\right) \circ\left(\operatorname{id}_{X} \square g\right)$. Often, when the map name is omitted from a morphism, the map denotes the identity morphism.

\section{Definition 6.1.3. Strict monoidal functor}

A strict monoidal functor between two strict monoidal categories $(\mathbf{V}, \square, I)$ and $\left(\mathbf{V}^{\prime}, \square^{\prime}, I^{\prime}\right)$ is a functor $F: \mathbf{V} \rightarrow \mathbf{V}^{\prime}$ such that

$$
m^{\prime} \circ(F \times F)=F \circ m \quad \text { and } \quad F \circ h=h^{\prime} .
$$

That is, the following diagrams commute.
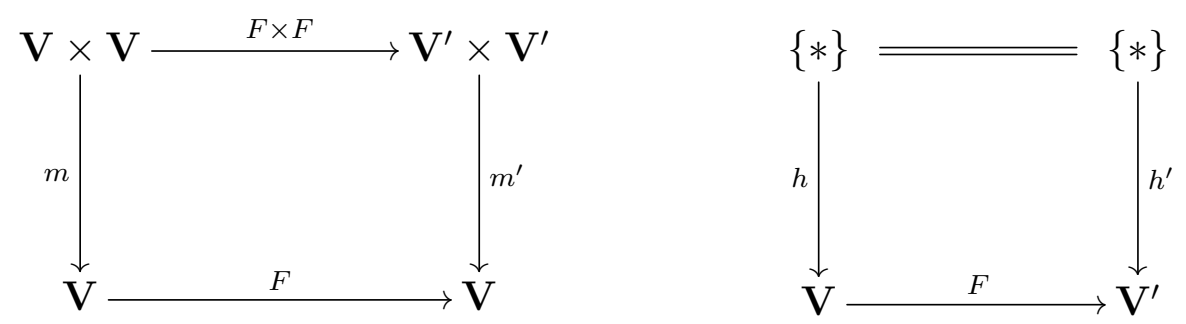
Therefore, for every pair of objects $X, Y$ in $\mathbf{V}, F(X \square Y)=F X \square^{\prime} F Y$, for every pair of morphisms $f, g$ in $\mathbf{V}, F(f \square g)=F f \square^{\prime} F g$, and also $F I=I^{\prime}$.

\section{Definition 6.1.4. Symmetric monoidal category}

A symmetric strictly monoidal category is a strictly monoidal category $(\mathbf{V}, \square, I)$ with a twist map,

$$
\tau_{X, Y}: X \square Y \cong Y \square X \quad \text { for every } X, Y \in \mathrm{ObV} \text {. }
$$

The morphism $\tau_{X, Y}$ will be drawn graphically as follows.

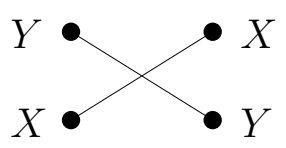

A twist map is an isomorphism, and has the following properties. Sometimes along with commuting diagrams, graphical diagrams have also been included.

1. Naturality for every pair of objects. That is, for every morphisms $f: X \rightarrow X^{\prime}$ and $g: Y \rightarrow Y^{\prime}$ in $\mathbf{V}$, the following diagram commutes.
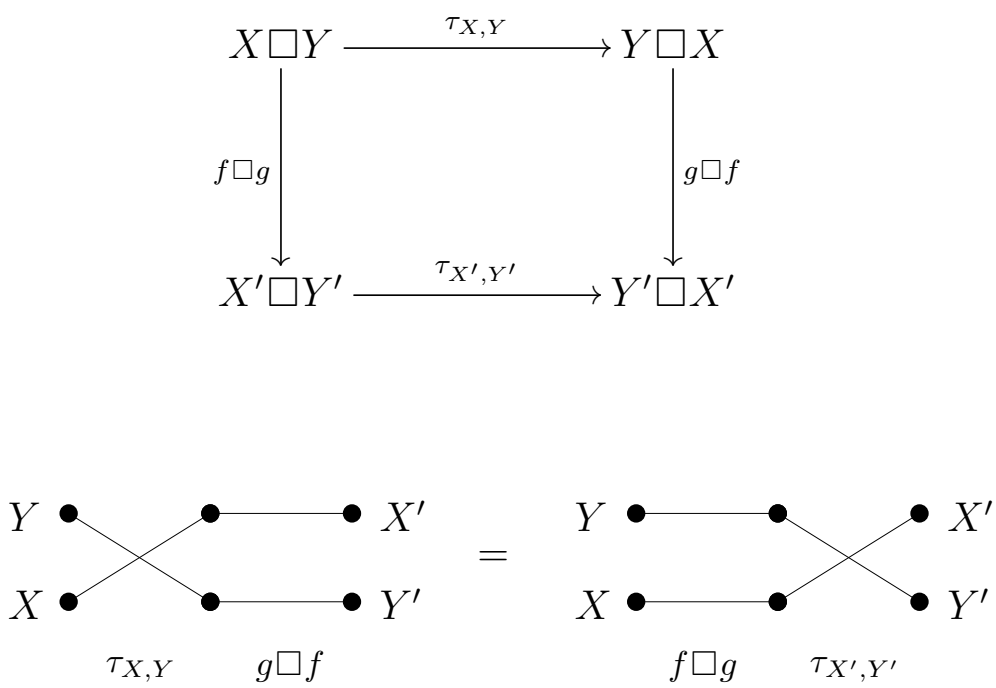
2. $\tau_{Y, X} \tau_{X, Y}=\mathrm{id}_{X \square Y}$
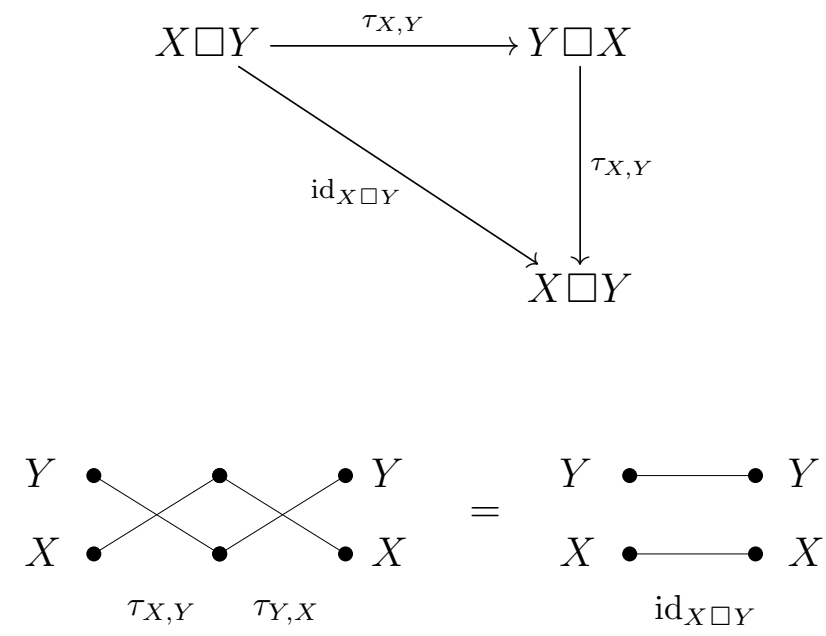

3. The Unit Law
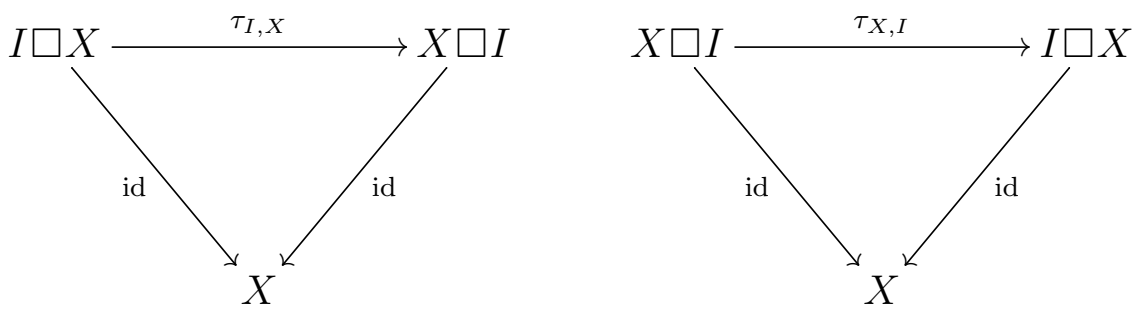

4. Associativity: $\left(\operatorname{id}_{Y} \square \tau_{X, Z}\right) \circ\left(\tau_{X, Y} \square \operatorname{id}_{Z}\right)=\tau_{X, Y \square Z}$ and $\left(\operatorname{id}_{X} \square \tau_{Y, Z}\right) \circ\left(\tau_{X, Z} \square \operatorname{id}_{Y}\right)=$ $\tau_{X \square Y, Z}$
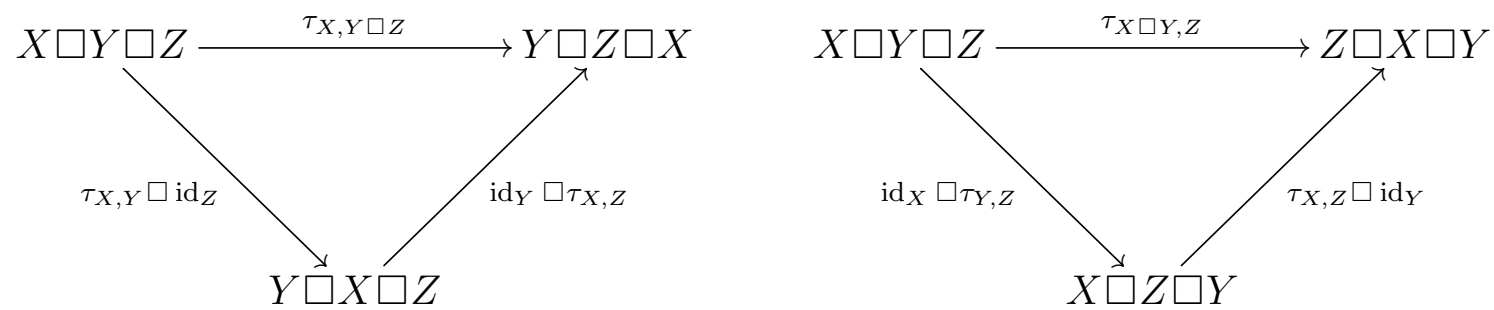

From conditions (2) and (4), the following equality holds, which we will not prove. 


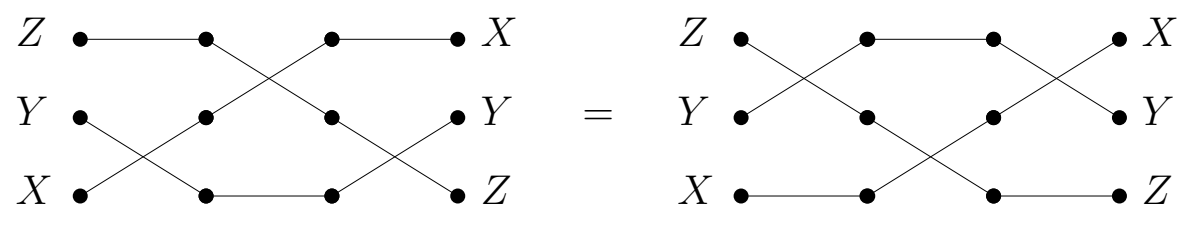

\section{Definition 6.1.5. Symmetric monoidal functor}

A symmetric strict monoidal functor between two symmetric strict monoidal categories $(\mathbf{V}, \square, I, \tau)$ and $\left(\mathbf{V}^{\prime}, \square^{\prime}, I^{\prime}, \tau^{\prime}\right)$ is a monoidal functor $F: \mathbf{V} \rightarrow \mathbf{V}^{\prime}$ such that for every pair of objects $X, Y$ in $\mathbf{V}$, the functor sends $\tau_{X, Y}$ to $\tau_{F X, F Y}^{\prime}$.

$$
F\left(\tau_{X, Y}\right)=\tau_{F X, F Y}^{\prime}
$$
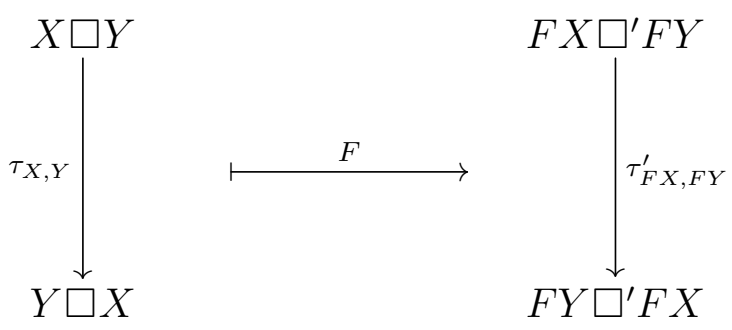

\section{Definition 6.1.6. Monoidal natural transformations}

Consider strict monoidal categories $(\mathbf{V}, \square, I)$ and $\left(\mathbf{V}^{\prime}, \square^{\prime}, I^{\prime}\right)$, and two strict monoidal functors $F, G: \mathbf{V} \rightrightarrows \mathbf{V}^{\prime}$. Recall a natural transformation from $F$ to $G$, written $u: F \rightarrow G$, is a collection of maps $\left\{u_{X}: F X \rightarrow G X\right\}_{X \in \mathrm{ObV}}$ in $\left(\mathbf{V}^{\prime}, \square^{\prime}, I^{\prime}\right)$ such that for every morphism $f: X \rightarrow Y$ in $\mathbf{V}$, the following square commutes in $\mathbf{V}^{\prime}$.

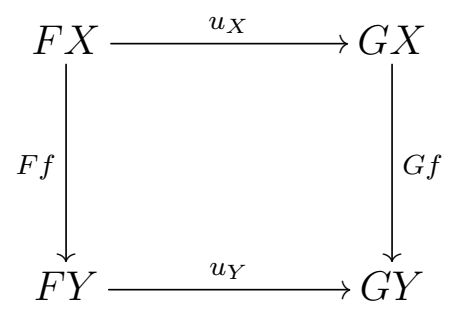


A (strict) monoidal natural transformation from $F$ to $G$ is a natural transformation $u: F \rightarrow G$, such that for every two objects $X, Y$ in $\mathbf{V}, u_{X} \square^{\prime} u_{Y}=u_{X \square Y}$, and $u_{I}=\operatorname{id}_{I^{\prime}}$. That is, the following diagrams commute.
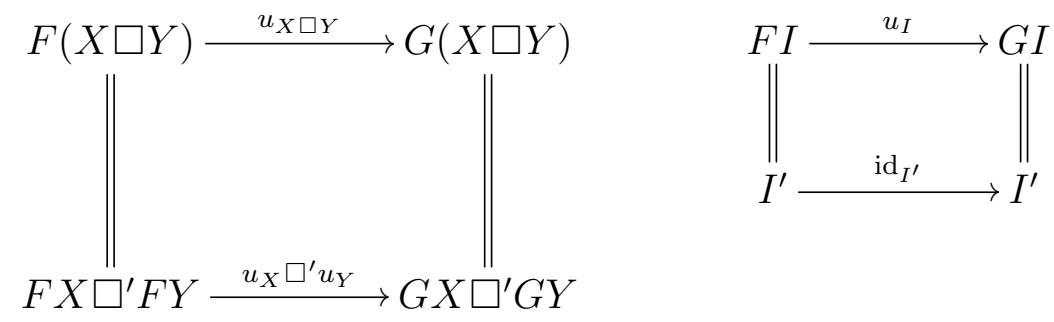

All the vertical equalities follow from the assumption that $F$ and $G$ are strict monoidal functors.

The collection of monoidal natural transformations, denoted $\operatorname{MonCat}\left(\mathbf{V}, \mathbf{V}^{\prime}\right)$, form a category where the objects are monoidal functors $\mathbf{V} \rightarrow \mathbf{V}^{\prime}$, and the morphisms are monoidal natural transformations.

Consider the category of only the symmetric monoidal functors in $\operatorname{MonCat}\left(\mathbf{V}, \mathbf{V}^{\prime}\right)$. This is a full subcategory of $\operatorname{MonCat}\left(\mathbf{V}, \mathbf{V}^{\prime}\right)$, denoted $\operatorname{SymMonCat}\left(\mathbf{V}, \mathbf{V}^{\prime}\right)$. The objects are only symmetric monoidal functors $\mathbf{V} \rightarrow \mathbf{V}^{\prime}$, and the morphisms are exactly the morphisms between these objects in $\operatorname{Mon} \operatorname{Cat}\left(\mathbf{V}, \mathbf{V}^{\prime}\right)$ (hence it is the full subcategory).

\subsubsection{Generating categories under the monoidal product}

Just like a group can be generated by a subset of the group, a monoidal category $\mathcal{C}$ can be generated by certain subsets of its objects, ObC. Of course, one could not define a generating set without the existence of a monoidal product, $m$. 
To illustrate this, we begin by looking at the most general example of the types of categories we want to look at. In this case, it is the simplex category $\Phi$. The category $\Phi$ is the skeleton of the category of finite sets, or FinSet.

The objects of $\Phi$ are given by natural numbers. That is, for $n \geq 1, \mathbf{n}:=\{0,1, \ldots, n-$ $1\}$ and $0:=\emptyset$. The morphisms of $\Phi$ are all set functions on the underlying sets. The monoidal product in $\Phi$ is the cardinal sums of cardinals, denoted + , and $+(m, n)=$ $m+n$. The neutral object is $\mathbf{0}$ (the empty set). The following equations assert that the monoidal product and neutral object are indeed part of a monoidal category. That is, + is associative and the cardinal sum of an object in $\Phi$ and $\mathbf{0}$ doesn't change the object.

$$
(\mathbf{k}+\mathbf{m})+\mathbf{n}=\mathbf{k}+(\mathbf{m}+\mathbf{n}) \quad \text { and } \quad \mathbf{0}+\mathbf{n}=\mathbf{n}=\mathbf{n}+\mathbf{0}
$$

Every $\mathbf{n} \in \mathrm{Ob} \Phi$ can be written as a disjoint union of $n$ many of the $\mathbf{1}$. Therefore, all objects in $\Phi$ can be written as a monoidal co-product of $\mathbf{1}$, and therefore $\mathbf{1}$ generates $\mathrm{ObV}$. Note that $\mathbf{0}$ is the unique initial object in $\Phi$, that is, it is the empty co-product of 1 .

Note that $\mathbf{0}$ is an initial object in $\Phi$, and + is the co-product in $\Phi$. This is in fact an example of the general case that categories with co-products and an initial object are monoidal.

For our purposes, it will be sufficient to know that all set maps on FinSet are cardinal sums of identity morphisms id, twist morphisms $t$, multiplication morphisms $\mu_{\Phi}$, and unit morphisms $\eta_{\Phi}$. In Set, $\mu_{\Phi}$ is the function which sends two elements to one element, $\eta_{\Phi}$ is the unique function from the empty set to a single element set, and the twist map $t$ is a permutation of a two element set. For more specifics, see [7]. 


\subsubsection{Monoids in monoidal categories}

\section{Definition 6.1.7. Monoids in strict monoidal categories}

Consider a monoidal category $(\mathbf{V}, \square, I)$. A monoid in $\mathbf{V}$ is an object $M \in \mathrm{ObV}$ together with two morphisms

$$
\mu: M \square M \rightarrow M \quad \text { and } \quad \eta: I \rightarrow M
$$

which satisfy $\mu\left(\mu \square \operatorname{id}_{M}\right)=\mu\left(\operatorname{id}_{M} \square \mu\right)$ and $\mu\left(\eta \square \operatorname{id}_{M}\right)=\operatorname{id}_{M}=\mu\left(\operatorname{id}_{M} \square \eta\right)$.
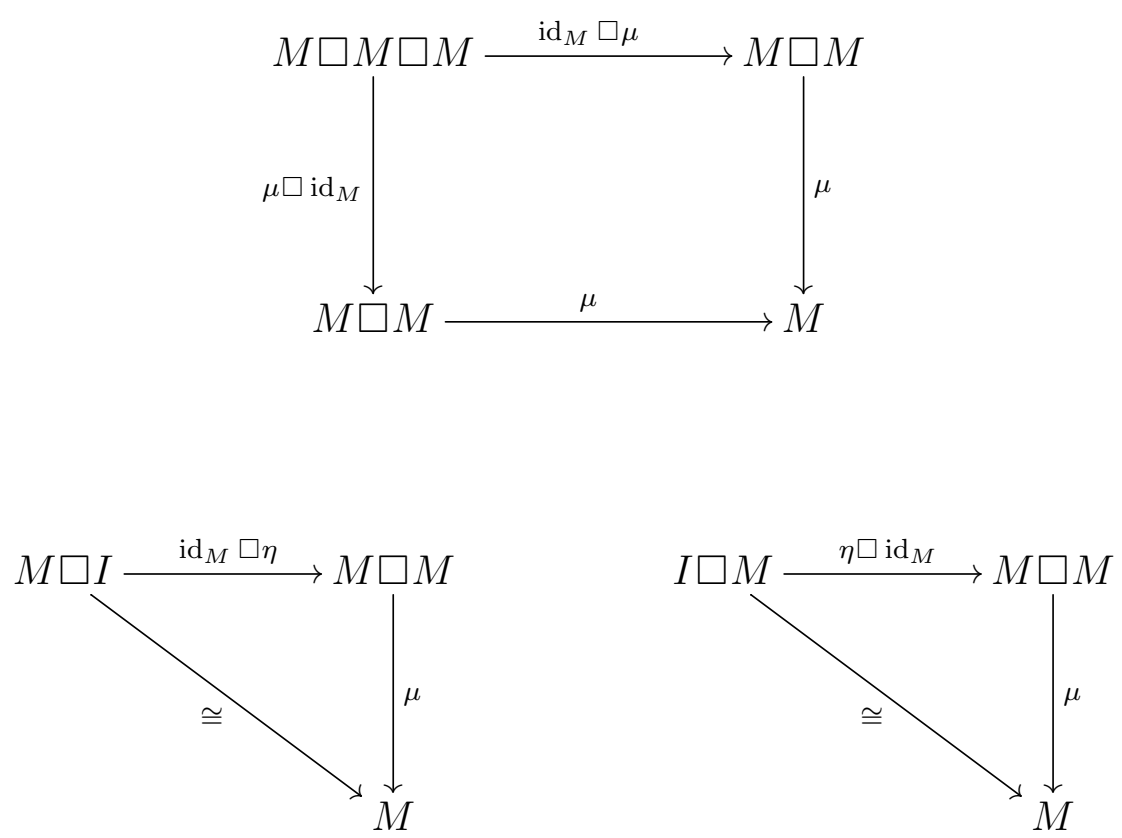

The top diagram implies associativity of multiplication, which can be shown as in the diagram below.

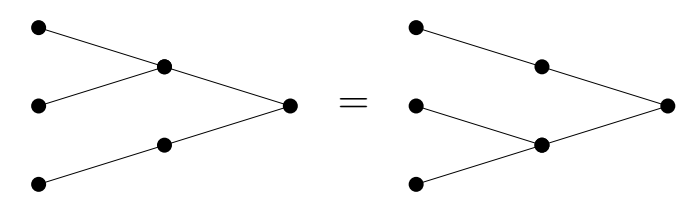

The bottom commutative diagrams imply the following, known as the unit identities. 


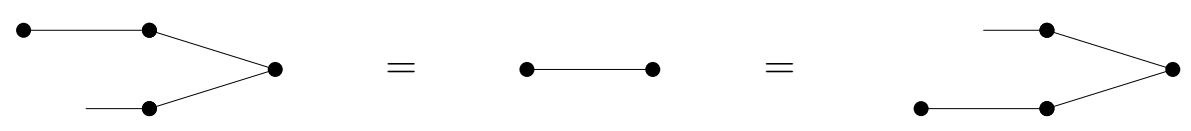

We denote a monoid in $\mathbf{V}$ as a triple with its multiplication map and unit map. That is, a monoid will be denoted $(M, \mu, \eta)$.

In the above diagrams, we have omitted labelling the dots. This is because it is clear in this context that every dot represents $M$, the monoidal object. The map $\mu$, represented graphically by $\supset$ is called the multiplication map and the map $\eta$, represented graphically by $\rightarrow$ is called the unit map.

\section{Definition 6.1.8. Monoid homomorphisms}

A monoid homomorphism, $\psi: M \rightarrow M^{\prime}$, is a morphism in a monoidal category $(\mathbf{V}, \square, I)$ between monoids $M, M^{\prime}$ such that their monoidal structure is preserved. That is, $\mu^{\prime}(\psi \square \psi)=\psi \mu$ and $\psi \eta=\eta^{\prime}$.
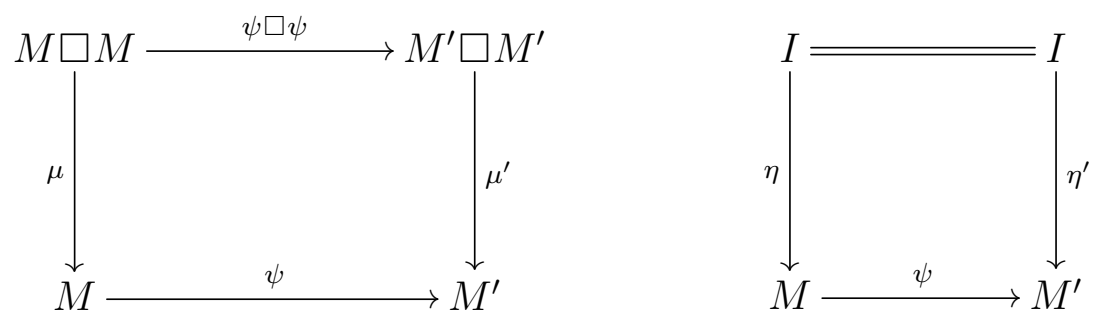

The collection of monoids in a monoidal category $(\mathbf{V}, \square, I)$ form a category, denoted $\operatorname{Mon}(\mathbf{V})$. The objects of this category are monoids $(M, \mu, \eta)$, and the morphisms are monoid homomorphisms.

\section{Definition 6.1.9. Commutative monoids}

Consider now a symmetric (strict) monoidal category $(\mathbf{V}, \square, I, \tau)$. A monoid $M$ in $\mathbf{V}$ is called a commutative monoid if the multiplication map $\mu$ is unaffected by pre-composing with the twist map $\tau$. That is, if

$$
\mu \tau=\mu .
$$


Note the subscript of $\tau_{M, M}$ is omitted. We will continue to do so when the monoid is clear from the context. So the following diagram commutes.
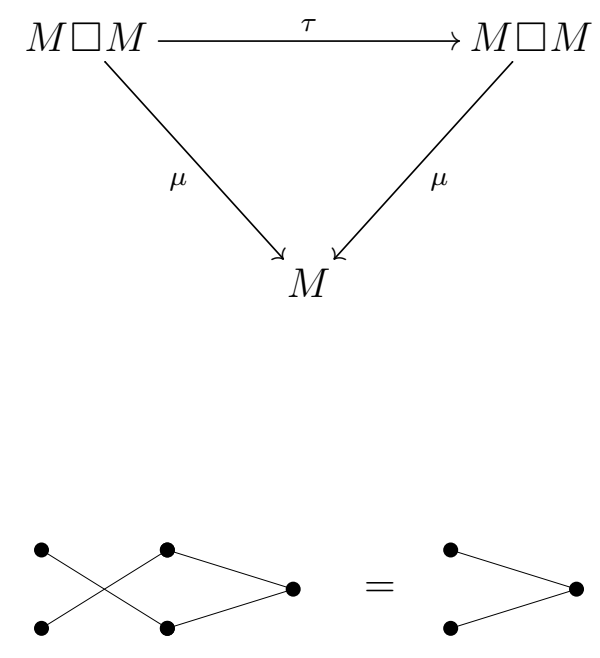

The collection of commutative monoids forms a category, written $\mathbf{c M o n}(\mathbf{V})$. The morphisms are monoid homomorphisms.

Recall from earlier in this section, $(\Phi,+, \mathbf{0}, t)$ is the simplex category described in Subsection 6.1.1. We now prove a theorem which demonstrates an isomorphism of categories that is a precursor to the main theorem of this chapter.

Theorem 6.1.10. Let $(\mathbf{V}, \square, I, \tau)$ be a symmetric monoidal category. Then there is an isomorphism of categories as follows.

$$
\begin{array}{clc}
\operatorname{SymMonCat}(\Phi, \mathbf{V}) & \cong \quad \operatorname{cMon}(\mathbf{V}) \\
{[F: \Phi \rightarrow \mathbf{V}]} & \mapsto\left(F \mathbf{1}, F \mu_{\Phi}, F \eta_{\Phi}, F t\right)
\end{array}
$$

Proof. We show this isomorphism of categories by defining a pair of functors $\mathcal{M}, \mathcal{N}$ which are isomorphisms of categories. Specifically, we check that $\mathcal{M}, \mathcal{N}$ are well defined functors and that $\mathcal{M} \circ \mathcal{N}=\mathrm{id}_{\mathbf{c M o n}(\mathbf{V})}$ and $\mathcal{N} \circ \mathcal{M}=\operatorname{id}_{\operatorname{SymMonCat}(\Phi, \mathbf{V})}$. 


$$
\begin{array}{rlc}
\mathcal{M}: \operatorname{SymMon} \operatorname{Cat}(\Phi, \mathbf{V}) & \rightarrow & \operatorname{cMon}(\mathbf{V}) \\
{[F: \Phi \rightarrow \mathbf{V}]} & \mapsto & \left(F \mathbf{1}, F\left(\mu_{\Phi}\right), F\left(\eta_{\Phi}\right), F(t)\right) \\
u: F \rightarrow G & \mapsto & \left(u_{1}: F \mathbf{1} \rightarrow G \mathbf{1}\right)
\end{array}
$$

We show that $\mathcal{M}$ is indeed a well defined functor. Suppose $F$ is a symmetric monoidal functor $\Phi \rightarrow \mathbf{V}$. First, $\left(F \mathbf{1}, F\left(\mu_{\Phi}\right), F\left(\eta_{\Phi}\right)\right)$ is a monoid with multiplication $F\left(\mu_{\Phi}\right): F \mathbf{1} \square F \mathbf{1} \rightarrow F \mathbf{1}$ and unit $F\left(\eta_{\Phi}\right): I \rightarrow F \mathbf{1}$, which follows as $F$ is monoidal so $F(\mathbf{1}+\mathbf{1})=F \mathbf{1} \square F \mathbf{1}$ and $I=F \mathbf{0}$. Since $F$ is a monoidal functor, the associativity of $F \mu_{\Phi}$ follows from the associativity of $\mu_{\Phi}$, and similarly for the unit relation.

Lastly, $F \mathbf{1}$ is a commutative monoid, since $F$ is a symmetric monoidal functor, for the twist map in $\Phi, t, F(t)=\tau$. Therefore, $F(t) F\left(\mu_{\Phi}\right)=F\left(t \mu_{\Phi}\right)=F \mu_{\Phi}$, as required.

To show $F$ is well defined on morphisms, we need to show $u_{\mathbf{1}}$ is a monoid homomorphism. Suppose $u: F \rightarrow G$ is a monoidal natural transformation. Since $u$ is monoidal, $u_{\mathbf{1}+\mathbf{1}}=u_{\mathbf{1}} \square u_{\mathbf{1}}$ and $F \mathbf{0}=G \mathbf{0}=I$, and also $F(\mathbf{1}+\mathbf{1})=F \mathbf{1} \square F \mathbf{1}$. By naturality of $u$ and the relations of the previous sentence, the following diagrams commute, which ensures that $u_{\mathbf{1}}$ is a monoid homomorphism from $F \mathbf{1}$ to $G \mathbf{1}$.
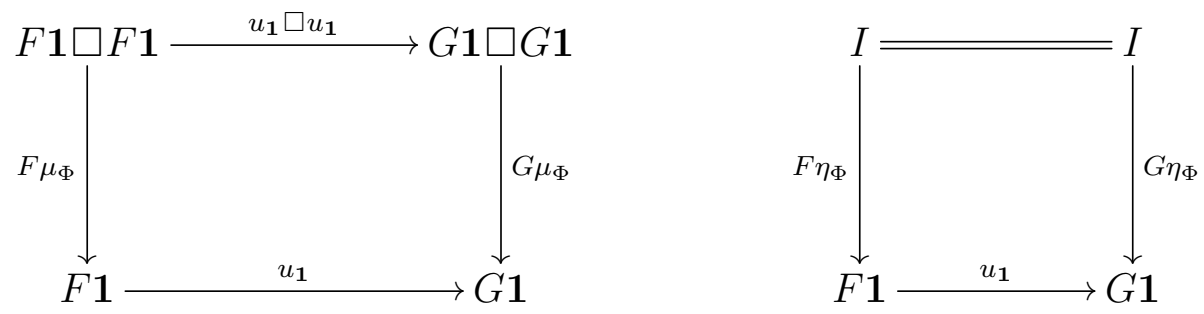


$$
\begin{aligned}
& \mathcal{N}: \operatorname{cMon}(\mathbf{V}) \quad \rightarrow \operatorname{SymMonCat}(\Phi, \mathbf{V}) \\
& \left(\begin{array}{c}
M \\
\mu \\
\eta \\
\tau
\end{array}\right) \quad \mapsto\left(\begin{array}{rlll}
1 & \mapsto & M \\
F: & \mu_{\Phi} & \mapsto & \mu \\
& \eta_{\Phi} & \mapsto & \eta \\
t & \mapsto & \tau
\end{array}\right) \\
& \left(\psi: M \rightarrow M^{\prime}\right) \mapsto \quad\left(u: F \rightarrow F^{\prime}\right)
\end{aligned}
$$

The definition of the functor $\mathcal{N}$ relies on the realisation that a functor can be defined on "generating objects and morphisms" of the domain category. For now, while it is clear the functor can be defined this way, it is not exactly clear what it means to generate the category $\Phi$, though discussed briefly earlier on in the section. For now we will take it on faith that we can do this, and discuss it further in the next section.

We now check this functor $\mathcal{N}$ is well defined on objects. The image of $M$ is a functor on objects that is defined by how it acts on the generating object of $\Phi, \mathbf{1}$. That is, since every object $\mathbf{n}$ in $\Phi$ can be written as a cardinal sum of the object $\mathbf{1}$, and the neutral object is sent to the neutral object, $F \mathbf{0}=I$.

$$
F(\mathbf{n})=F(\underbrace{\mathbf{1}+\cdots+1}_{n}):=\underbrace{F \mathbf{1} \square \cdots \square F \mathbf{1}}_{n}=\underbrace{M \square \cdots \square M}_{n}
$$

This extends to morphisms of $\Phi . F$ is well defined on morphisms as all morphisms in $\Phi$ are composites of cardinal sums of twists, identity arrows, multiplication arrows and units. Therefore, $F$ is a well defined functor. Equation (6.1) ensures $F$ is a monoidal functor, that is, that $F(\mathbf{m}+\mathbf{n})=F \mathbf{m} \square F \mathbf{n}$, and it follows that for $f, g \in \operatorname{Mor} \Phi, F(f+g)=F f \square F g . F$ is also symmetric as the twist map is brought to the twist map: $F t=\tau$.

Next we check that $\mathcal{N}$ is well defined on morphisms. Suppose $\psi: M \rightarrow M^{\prime}$ is a monoidal homomorphism, and let $\mathcal{N}(M, \mu, \eta)=F$ and $\mathcal{N}\left(M^{\prime}, \mu^{\prime}, \eta^{\prime}\right)=G$. The com- 
muting diagram on the left is as required for $\psi$ to be a monoidal homomorphism. If one replaces the objects and morphisms to be in the image of $F$ and defines $u_{\mathbf{1}}:=\psi$, then it becomes the commuting diagram on the right, which is one of the conditions for $u$ to be a natural transformation.
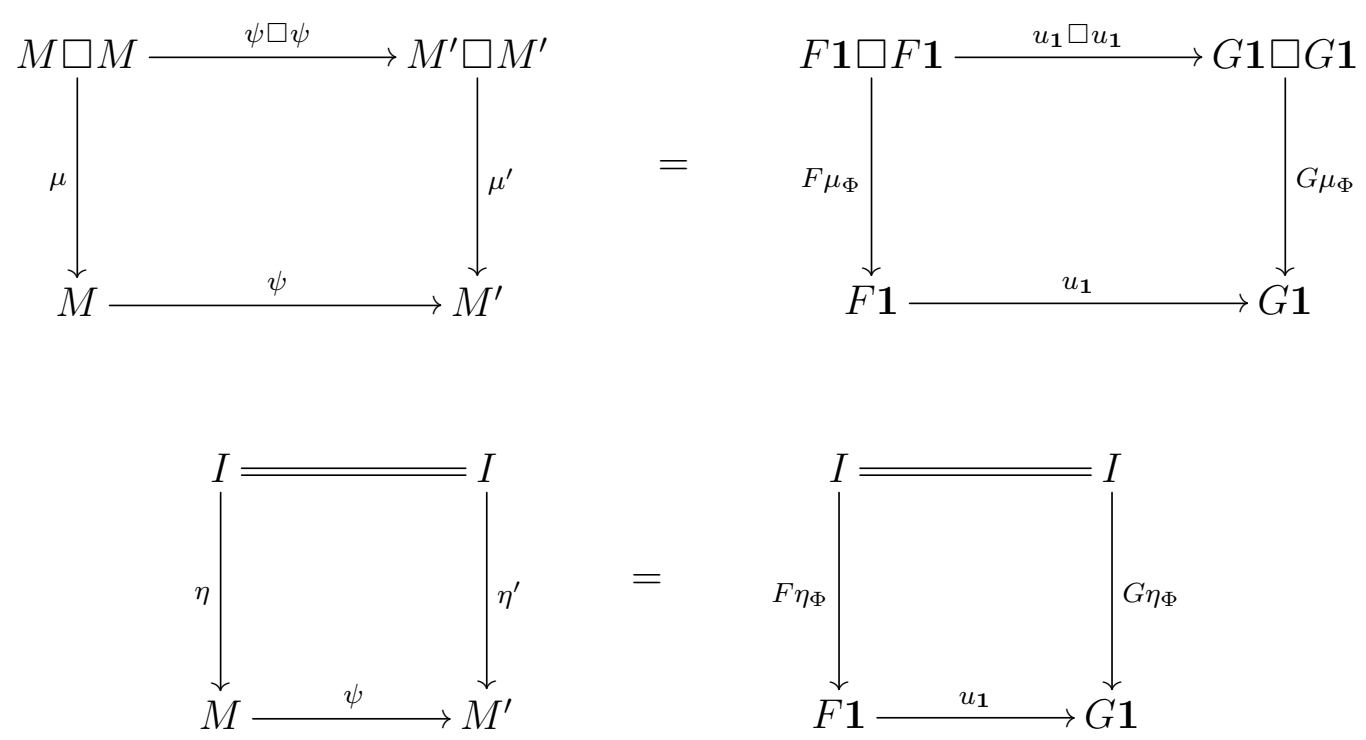

The above diagrams show that $u$ is natural on $\mu_{\Phi}$ and $\eta_{\Phi}$. Naturality of $\tau$ in $V$ means that $(\psi \square \psi) \circ \tau=\tau \circ(\psi \square \psi)$, so clearly since $F t=G t=\tau, u$ is natural on $t$.

Let $u_{\mathbf{n}}=\underbrace{\psi \square \cdots \square \psi}_{n}$. We will check that $u$ is natural for all morphisms in $\Phi$. Suppose $f: \mathbf{m} \rightarrow \mathbf{n}$ in $\operatorname{Mor} \Phi$, and hence is a monoidal product of $\mu_{\Phi}, \eta_{\Phi}, t$, and the identity morphisms. One can rearrange $u_{\mathbf{n}} \circ F f$ to be a cardinal sum of $u_{\mathbf{2}} \circ F \mu_{\Phi}, u_{\mathbf{1}} \circ F \eta_{\Phi}$, $u_{2} \circ F t$, and $u_{1} \circ \mathrm{id}$. As mentioned previously, $u_{1}$ or $u_{1} \square u_{1}$ (respectively) commutes with each of these $G \mu_{\Phi} \circ u_{2}, G \eta_{\Phi}, G t \circ u_{\mathbf{2}}$. Depending on the map $f$, one may have to rearrange the sums between applying naturality for each $\mu_{\Phi}$, etc.. One can do this for compositions of maps. Therefore, rearranging in terms of $G f$, this shows that $u_{\mathbf{n}} \circ F f=G f \circ u_{\mathbf{m}}$, so $u$ is indeed a monoidal natural transformation. 
It only remains to show that the two functors $\mathcal{M}$ and $\mathcal{N}$ are mutual inverses. Firstly, $\mathcal{M N}=\operatorname{id}_{\mathbf{c M o n}(\mathbf{V})}$ as

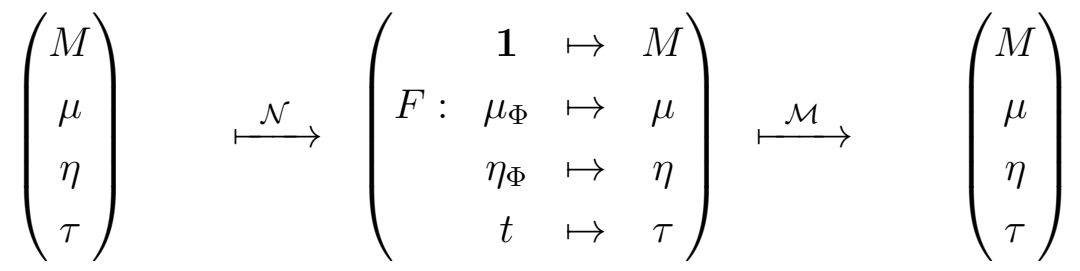

$$
\begin{aligned}
& \left(\psi: M \rightarrow M^{\prime}\right) \stackrel{\mathcal{N}}{\longmapsto}\left(\begin{array}{c}
u: F \rightarrow F^{\prime} \\
\text { where } u_{1}=\psi
\end{array}\right) \stackrel{\mathcal{M}}{\longmapsto}\left(\psi: M \rightarrow M^{\prime}\right)
\end{aligned}
$$

$\mathcal{N} \mathcal{M}=\operatorname{id}_{\text {SymMonCat }(\Phi, \mathbf{V})}$.

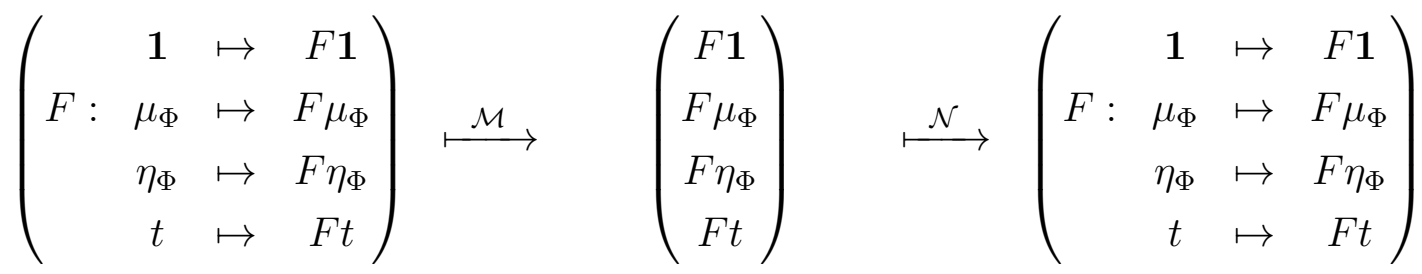

$$
\begin{aligned}
& \left(u: F \rightarrow F^{\prime}\right) \quad \stackrel{\mathcal{M}}{\longmapsto}\left(u_{1}: F \mathbf{1} \rightarrow F^{\prime} \mathbf{1}\right) \stackrel{\mathcal{N}}{\longmapsto} \quad\left(u: F \rightarrow F^{\prime}\right)
\end{aligned}
$$

as required.

We now begin to define the dual concept of a monoid.

\section{Definition 6.1.11. Co-monoids}

Let $(\mathbf{V}, \square, I)$ be a strict monoidal category. A co-monoid is an object in $\mathbf{V}$ with two morphisms

$$
\delta: M \rightarrow M \square M \quad \text { and } \quad \epsilon: M \rightarrow I
$$


which satisfy $\left(\delta \square \operatorname{id}_{M}\right) \delta=\left(\operatorname{id}_{M} \square \delta\right) \delta$ and $\delta\left(\epsilon \square \operatorname{id}_{M}\right)=\operatorname{id}_{M}=\delta\left(\operatorname{id}_{M} \square \epsilon\right)$.
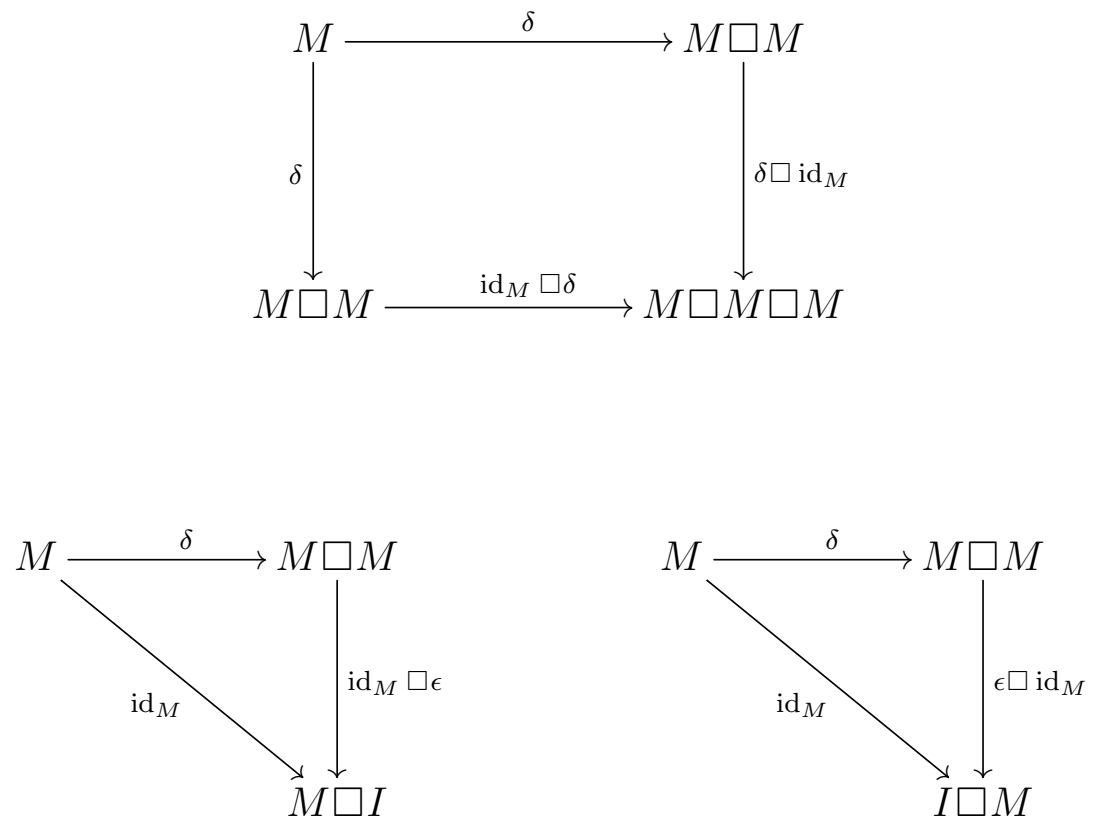

The top diagram implies co-associativity.

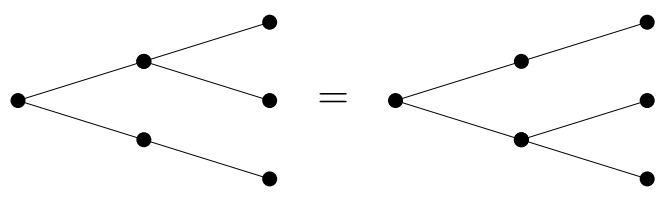

The bottom commutative diagrams imply the following co-unit relations.

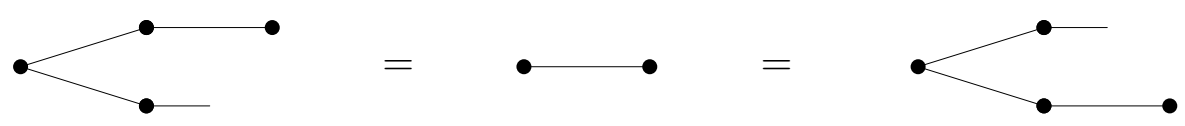

A co-monoid homomorphism, $\psi: M \rightarrow M^{\prime}$, is a morphism in a monoidal category $(\mathbf{V}, \square, I)$ between co-monoids $M, M^{\prime}$ such that their monoidal structure is preserved. 
That is, $\delta^{\prime} \psi=(\psi \square \psi) \delta$ and $\epsilon=\epsilon^{\prime} \psi$.
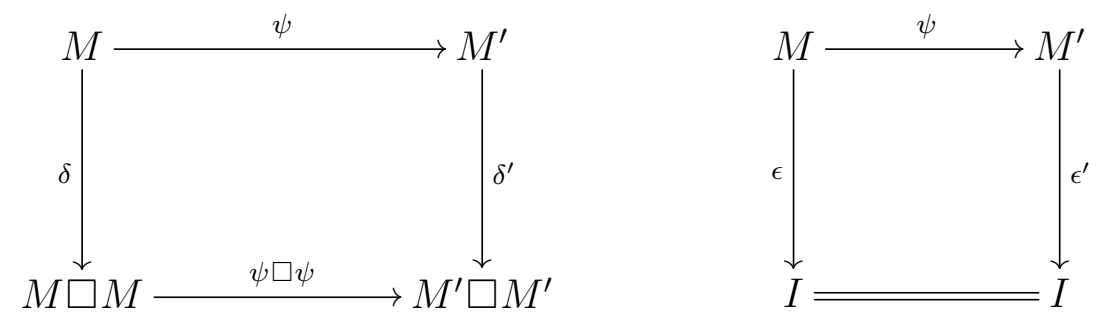

Like $\operatorname{Mon}(\mathbf{V})$, the collection of co-monoids in a monoidal category $(\mathbf{V}, \square, I)$ forms a category, denoted $\operatorname{Comon}(\mathbf{V})$. The objects of this category are co-monoids $(M, \delta, \epsilon)$, and the morphisms are co-monoid homomorphisms.

\section{Definition 6.1.12. Co-commutative co-monoids}

As previously, consider a symmetric strict monoidal category $(\mathbf{V}, \square, I, \tau)$. A comonoid $M$ in $\mathbf{V}$ is called a co-commutative co-monoid if the co-multiplication map $\delta$ commutes with the twist map $\tau$. That is, if

$$
\tau \delta=\delta
$$

So the following diagram commutes.
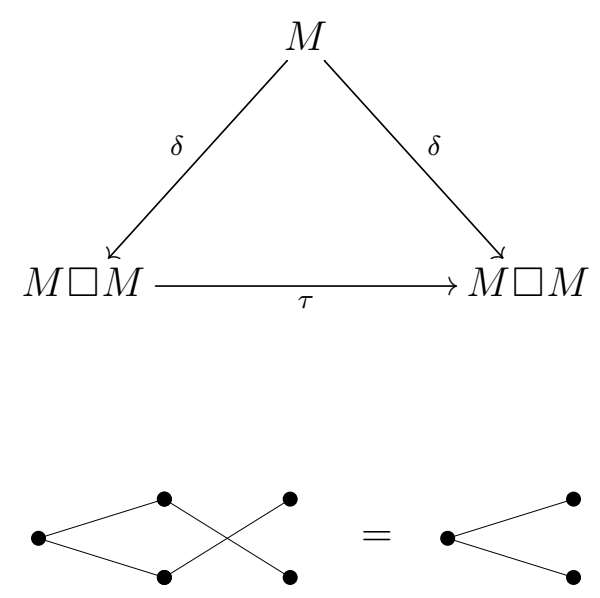

The collection of co-commutative co-monoids forms a category, written $\mathbf{c C o m o n}(\mathbf{V})$. The morphisms are co-monoid homomorphisms. 
For a category $C$, recall its opposite category is the category with the same objects as $C, \mathrm{ObC}$, and morphisms $f^{\mathrm{op}}$, which are in one to one correspondence with the morphisms $f$ of $C$. For a morphism $f: a \rightarrow b$ in $C$, the opposite morphism has the domain and co-domain swapped, that is $f^{\text {op }}: b \rightarrow a$. This opposite category is denoted $C^{\mathrm{op}}$.

Consider the opposite category $\Phi^{\mathrm{op}}$. In this category, we have $\mu_{\Phi}^{\mathrm{op}}=\delta_{\Phi \mathrm{op}}, \eta_{\Phi}^{\mathrm{op}}=\epsilon_{\Phi^{\mathrm{op}}}$ and $t^{\mathrm{op}}=t$. Since associativity of $\mu_{\Phi}$ implies co-associativity of $\delta_{\Phi \mathrm{op}}$, and all other relations on morphisms $\mu_{\Phi}, \eta_{\Phi}, t$ are the dual to the relations of $\delta_{\Phi}, \epsilon_{\Phi}, t$. With this in mind, it follows that there is a dual of Theorem 6.1.10, that $\operatorname{SymMonCat}\left(\Phi^{\mathrm{op}}, \mathbf{V}\right) \cong$ $\operatorname{cComon}(\mathbf{V})$.

Theorem 6.1.13. The category $(\mathbf{c M o n V}, \square, I, \tau)$ is a symmetric monoidal category. That is, if $M, M^{\prime}$ are objects in $\mathbf{c M o n} \mathbf{V}$, then $M \square M^{\prime}$ is also in $\mathbf{c M o n V}$.

Proof. Note that $I$ is in $\mathbf{c M o n V}$, where multiplication and the unit map are identites on $I$. All we are required to do is show there is a multiplication map and unit map for $M \square M^{\prime}$ such that multiplication is associative and the unit relation holds.

Let multiplication $\mu_{M \square M^{\prime}}:\left(M \square M^{\prime}\right) \square\left(M \square M^{\prime}\right) \rightarrow M \square M^{\prime}$ and the unit map $\eta_{M \square M^{\prime}}$ : $I \rightarrow M \square M^{\prime}$ be defined as follows.

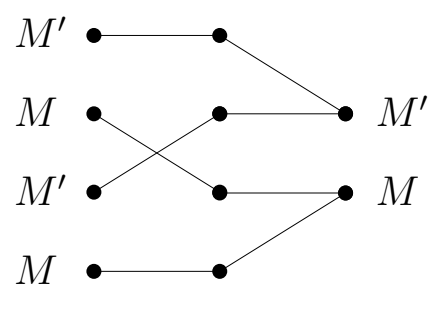

$\mu_{M \square M^{\prime}}$
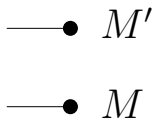

$\eta_{M \square M^{\prime}}$

First recall the following, which follows from naturality of the twist map and that $X \square I=X=I \square X$ for every object $X$ in $\mathbf{V}$. 

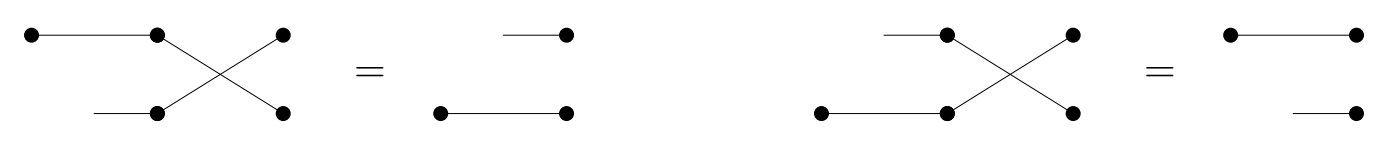

The unit relation holds, as follows.

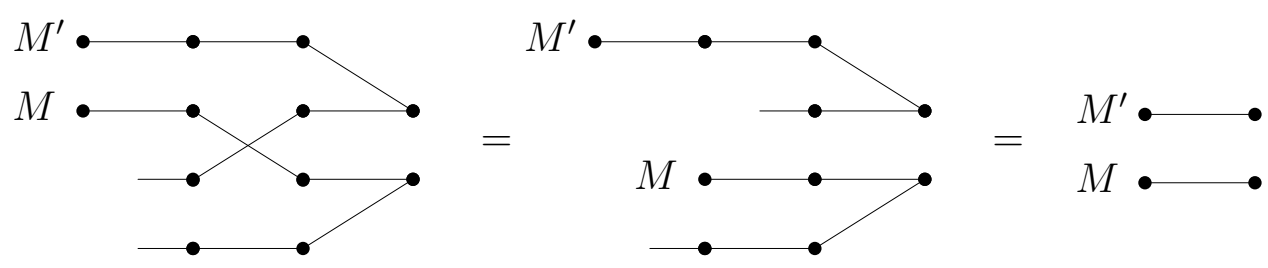

Now we show associativity of multiplication. First note the following relations hold by the naturality of the twist map $\tau$.

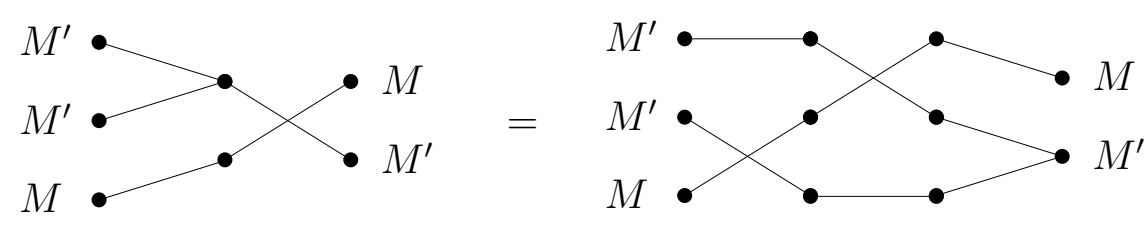

Now for the associativity of $\mu_{M \square M^{\prime}}$. 

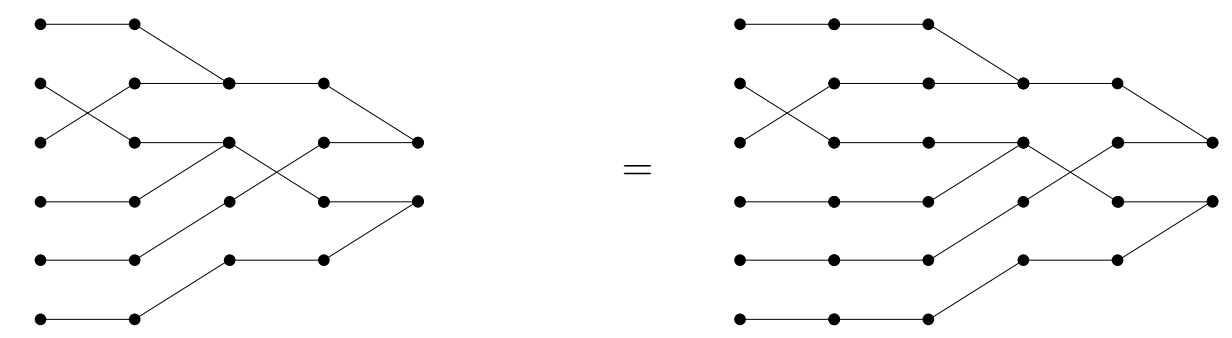

naturality of $\tau$

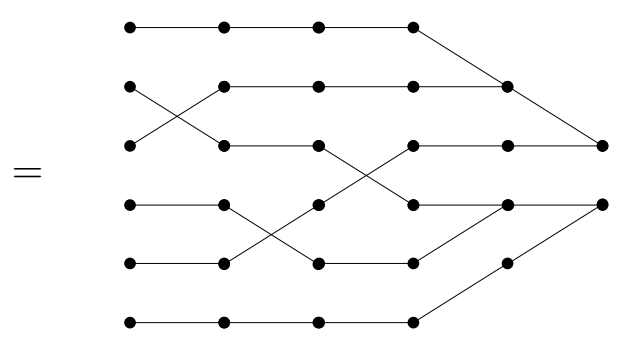

by associativity of multiplication and rearranging the twist maps and identity maps

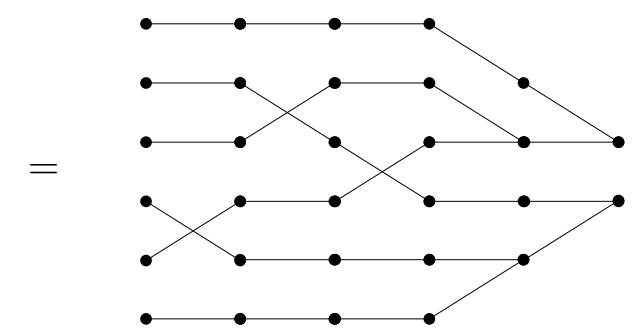

naturality of $\tau$

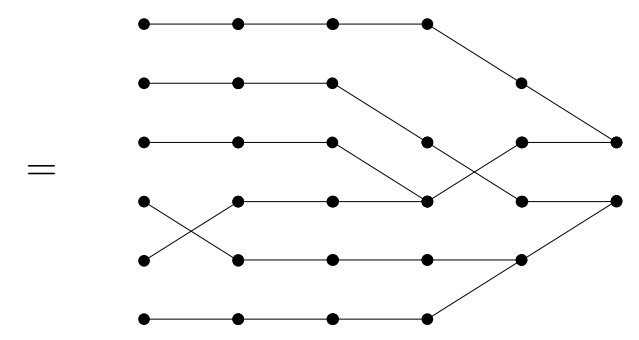

Rearrangement of identity maps

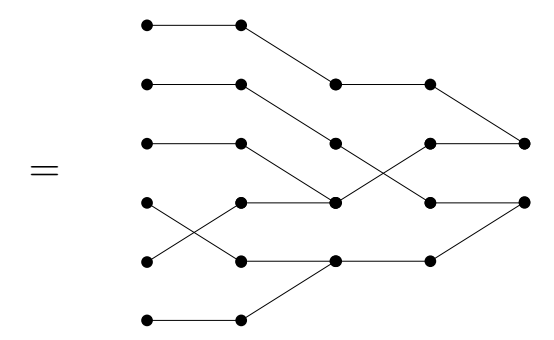


The dual category cComonV can also be shown to have the same property.

\subsection{Frobenius objects}

\section{Definition 6.2.1. Frobenius objects}

A Frobenius object in a (strict) monoidal category $(\mathbf{V}, \square, I)$ is an object $A$ with the following morphisms.

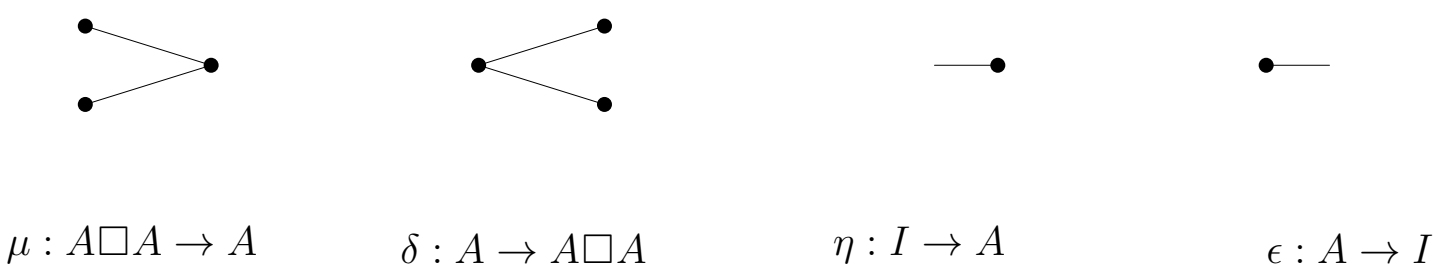

With the following unit and co-unit relations,

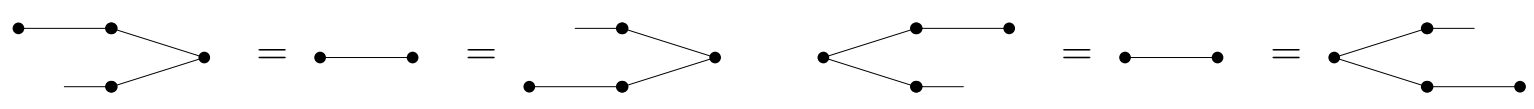

and the Frobenius relation

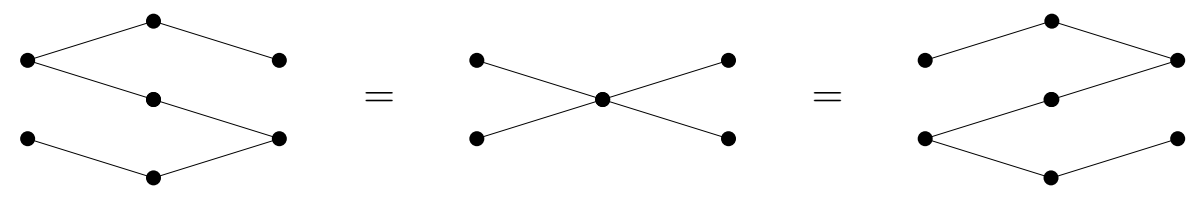

where each dot represents $A$. 
Lemma 6.2.2. A Frobenius object is both a monoid and a co-monoid in $\mathbf{V}$. That is, the "multiplication" map $\mu$ is associative and the "co-multiplication" map $\delta$ is coassociative.

Proof. In the spirit of this section, we show this proof graphically (as done by [7]).

First, we find an equivalent map to $\mu$.

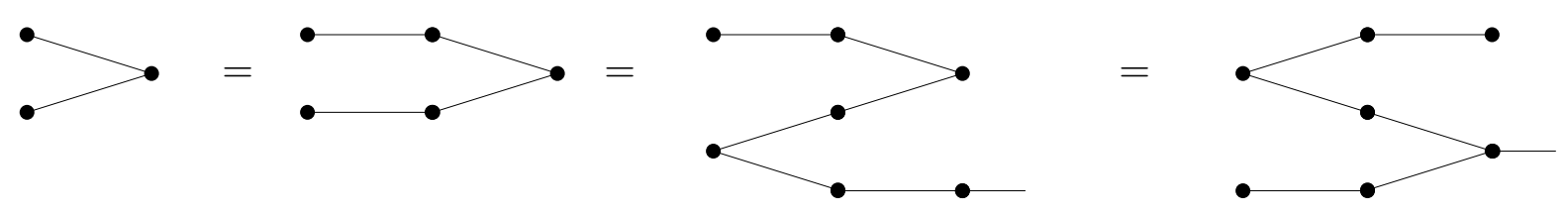

The second equality uses the co-unit relation, and the third equality uses the Frobenius relation.

Now we show associativity.
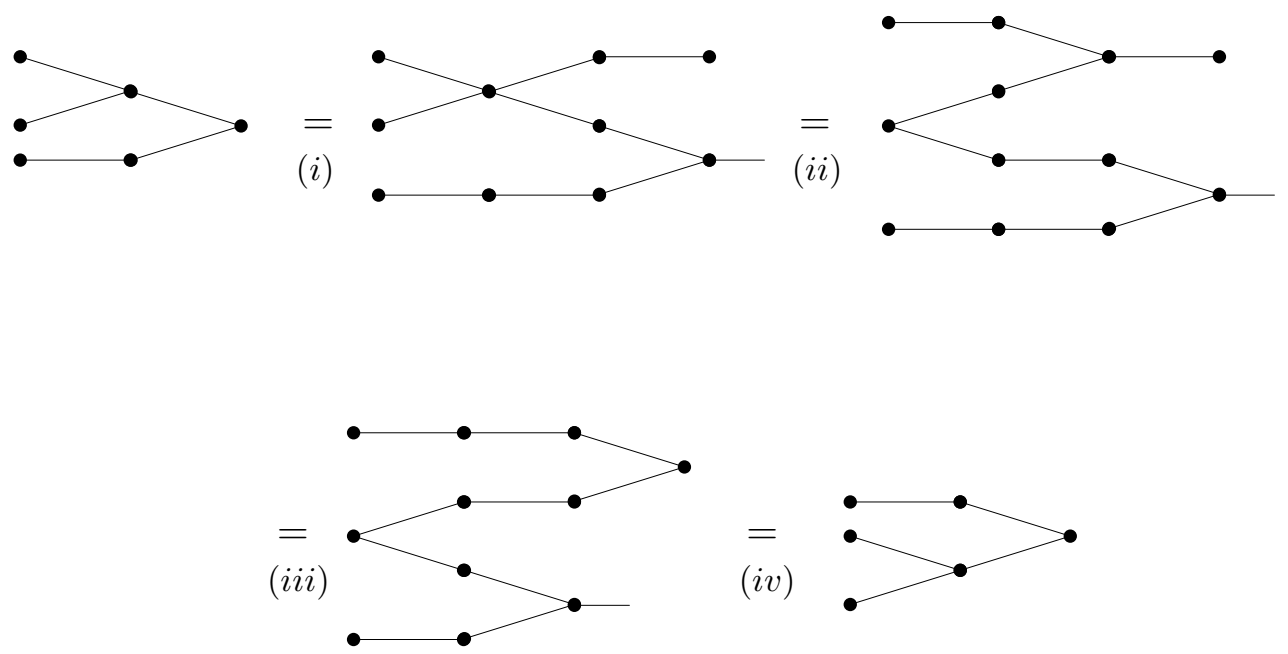

The third equality is simply a rearrangement of the monoidal products with identity maps. The first and fourth equalities use the substitution for multiplication, $\mu$, shown 
above, and the second quality uses the Frobenius relation.

The proof of co-associativity is exactly the dual of this proof.

Next we prove a lemma which we will use to show that $M$ is commutative if and only if it is co-commutative.

Lemma 6.2.3. For an object $M$ in FrobV, the multiplication map $\mu$ is the unique map $M \square M \rightarrow M$ that with the unit $\eta$ satisfies the unit relation and satisfies the Frobenius relation.

Dually, the co-multiplication map $\delta$ is the unique map $M \rightarrow M \square M$ that has counit $\epsilon$ and satisfies the the Frobenius relation.

Proof. This proof follows the method of [7], and is done in three parts. We call the following relation the snake relation, which follows easily from the unit, co-unit, and Frobenius relations.

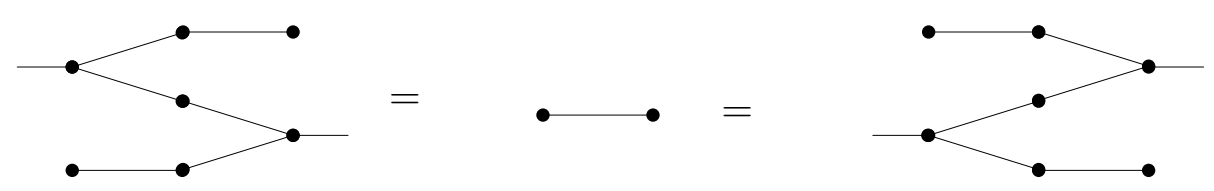

Firstly, we show that $\beta:=\epsilon \mu$ which satisfies the snake relation is unique. Suppose $\xi: M \square M \rightarrow I$ also satisfies the snake relation.

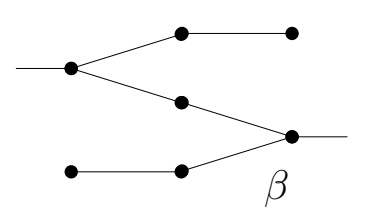

(i)

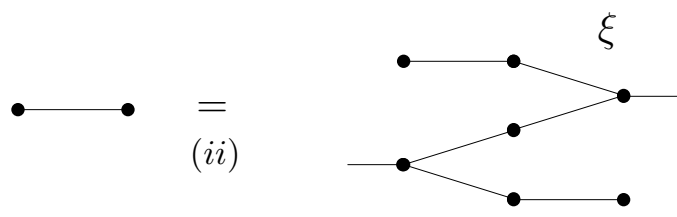

Consider now the right equality, (ii), and compose with $\beta$ on the right. After a 
rearrangement with identity maps, we get the equality $(i i i)$. The equality $(i v)$ follows from $(i)$ above .

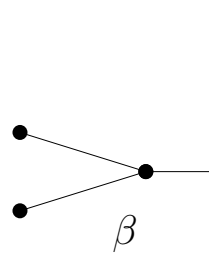

$\beta$

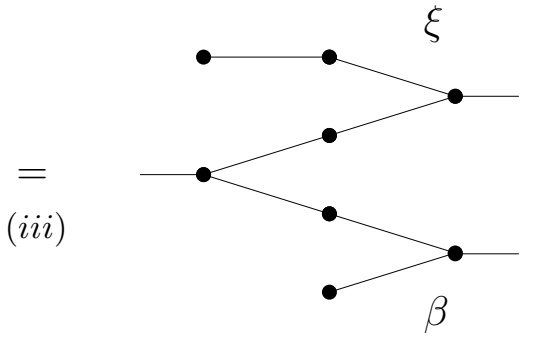

$=$

$(i v)$

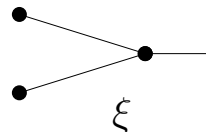

Next, we show that if $\omega: M \square M \rightarrow M$ satisfies the Frobenius relation and unit relation, then $\beta=\epsilon \omega$. In other words, because $\epsilon \omega$ satisfies the snake relation, and by the uniqueness of $\beta$ shown previously, $\beta=\epsilon \omega$.

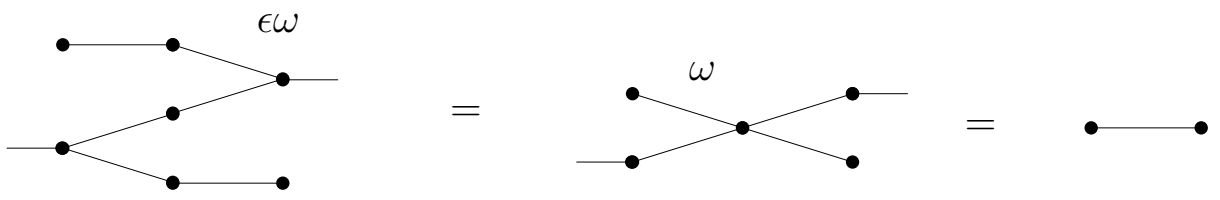

Therefore, since $\epsilon \omega$ satisifies the snake relation, we have $\beta=\epsilon \omega$. This is true for any $\omega$ that satisfies the Frobenius relation and the unit relation, which is true for $\mu$ as well, and hence $\epsilon \omega=\epsilon \mu$. The final step demonstrates that $\mu$ is the unique morphism that satisfies the Frobenius relation and the unit relation.
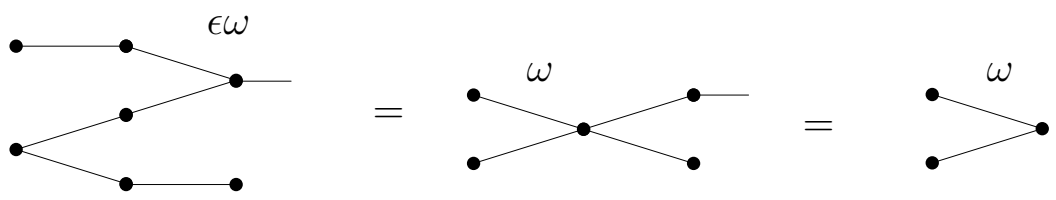

II
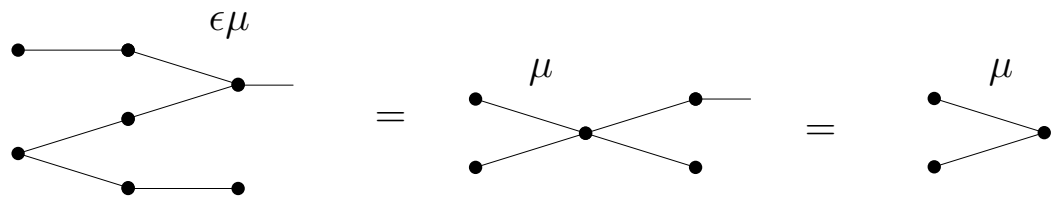
So $\omega=\mu$, as required. A dual proof shows co-multiplication, $\delta$, is unique.

\section{Definition 6.2.4. Commutative Frobenius objects}

A commutative Frobenius object in a symmetric monoidal category $(\mathbf{V}, \square, I, \tau)$ is a Frobenius object which is also a commutative monoid. That is, it satisfies $\mu \tau=\mu$.

Similarly, a co-commutative Frobenius object is a Frobenius object which is also a co-commutative co-monoid, and therefore satisifies $\tau \delta=\tau$.

Lemma 6.2.5. A Frobenius object is commutative if and only if it is co-commutative.

Proof. Suppose the following co-commutativity relation holds for a Frobenius object $M$.

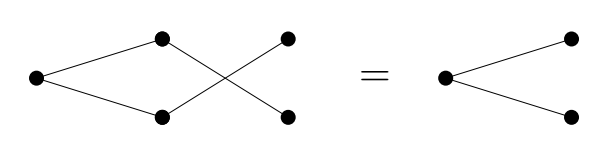

We will demonstrate that $M$ is also commutative by showing that $\mu \tau$ satisfies the co-unit relation and Frobenius relation, and then applying Lemma 6.2.3 which asserts that multiplication is unique, proving that $\mu \tau=\mu$.

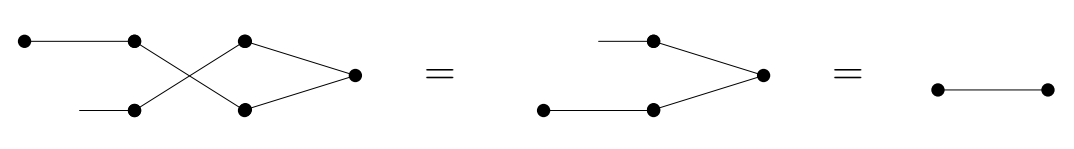

The first equality holds by the naturality of $\tau$ with $\eta \square$ id, and the second relation holds by the unit relation of $\mu$. It is identical to show $\mu \tau$ satisfies the other unit relation $\mu \tau(\operatorname{id} \square \eta)=$ id. Next we show that $\mu \tau$ satisfies the Frobenius relation. 

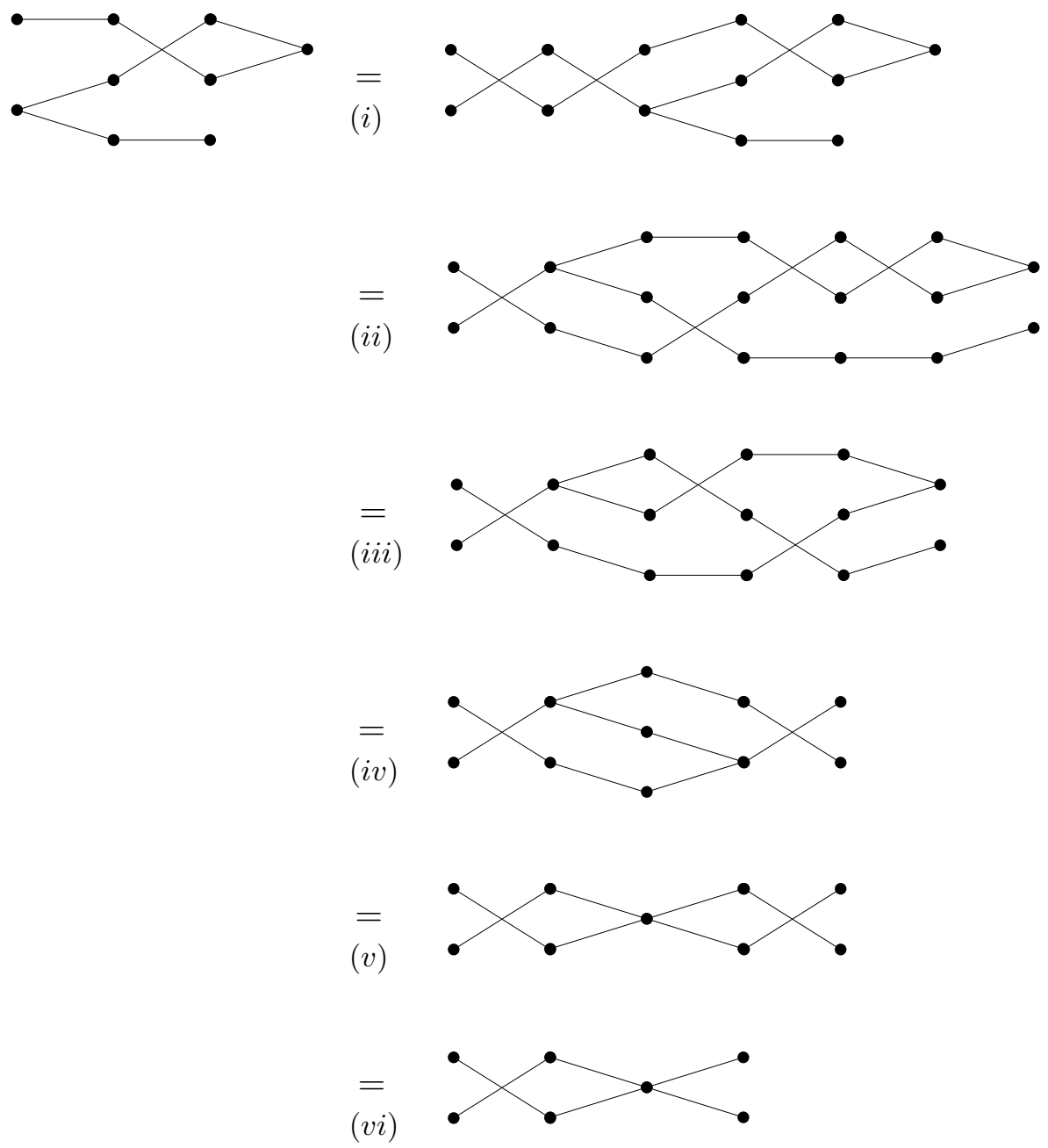

The equality $(i)$ follows from $\tau \tau=\mathrm{id}$. The second and fourth equalities are from naturality in $\tau$. The third equality uses the commutativity of $\delta$, and also $\tau \tau=$ id. The fifth equality uses the Frobenius relation. This shows $\mu \tau$ satisfies the Frobenius relation.

\section{Definition 6.2.6. Morphisms of Frobenius objects}

A morphism of Frobenius objects $(M, \mu, \delta, \eta, \epsilon),\left(M^{\prime}, \mu^{\prime}, \delta^{\prime}, \eta^{\prime}, \epsilon^{\prime}\right)$ in a symmetric monoidal category $(\mathbf{V}, \square, I, \tau)$ is a morphism $\psi: M \rightarrow M^{\prime}$ in $\mathbf{V}$ which is also a monoid homo- 
morphism and a co-monoid homomorphism. That is, it satisfies the following four equations.

$$
\begin{array}{cc}
\psi \mu=\mu^{\prime}(\psi \square \psi) & \delta^{\prime} \psi=(\psi \square \psi) \delta \\
\psi \eta=\eta^{\prime} & \epsilon^{\prime} \psi=\epsilon
\end{array}
$$

The collection of commutative Frobenius objects (and therefore co-commutative Frobenius objects) in a category $\mathbf{V}$ forms a category which is denoted $\mathbf{c F r o b V}$.

Corollary 6.2.7. The symmetric monoidal category (cFrobV $, \square, I, \tau)$ is a monoidal category. That is, if $M, M^{\prime}$ are objects in $\mathbf{c F r o b V}$, then $M \square M^{\prime}$ is also in $\mathbf{c F r o b V}$.

Proof. All we need to show is that the Frobenius relation holds for $M \square M^{\prime}$, as we have shown all other relations, the unit, the co-unit, associativity and co-associativity hold in Theorem 6.1.13, 


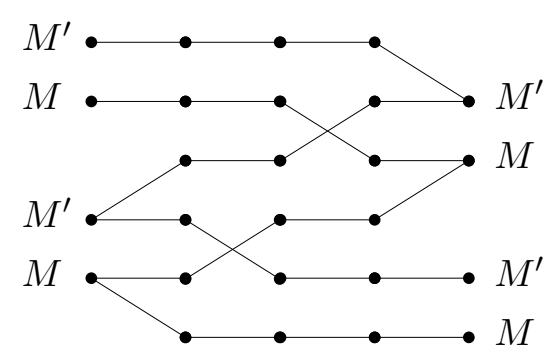

naturality of $\tau$

Since $\tau \tau=\mathrm{id}$, and a rearrangement

Since $\tau \tau=\mathrm{id}$, and a rearrangement

By the Frobenius relation for $M$ and $M^{\prime}$, and a rearrangement, as required.
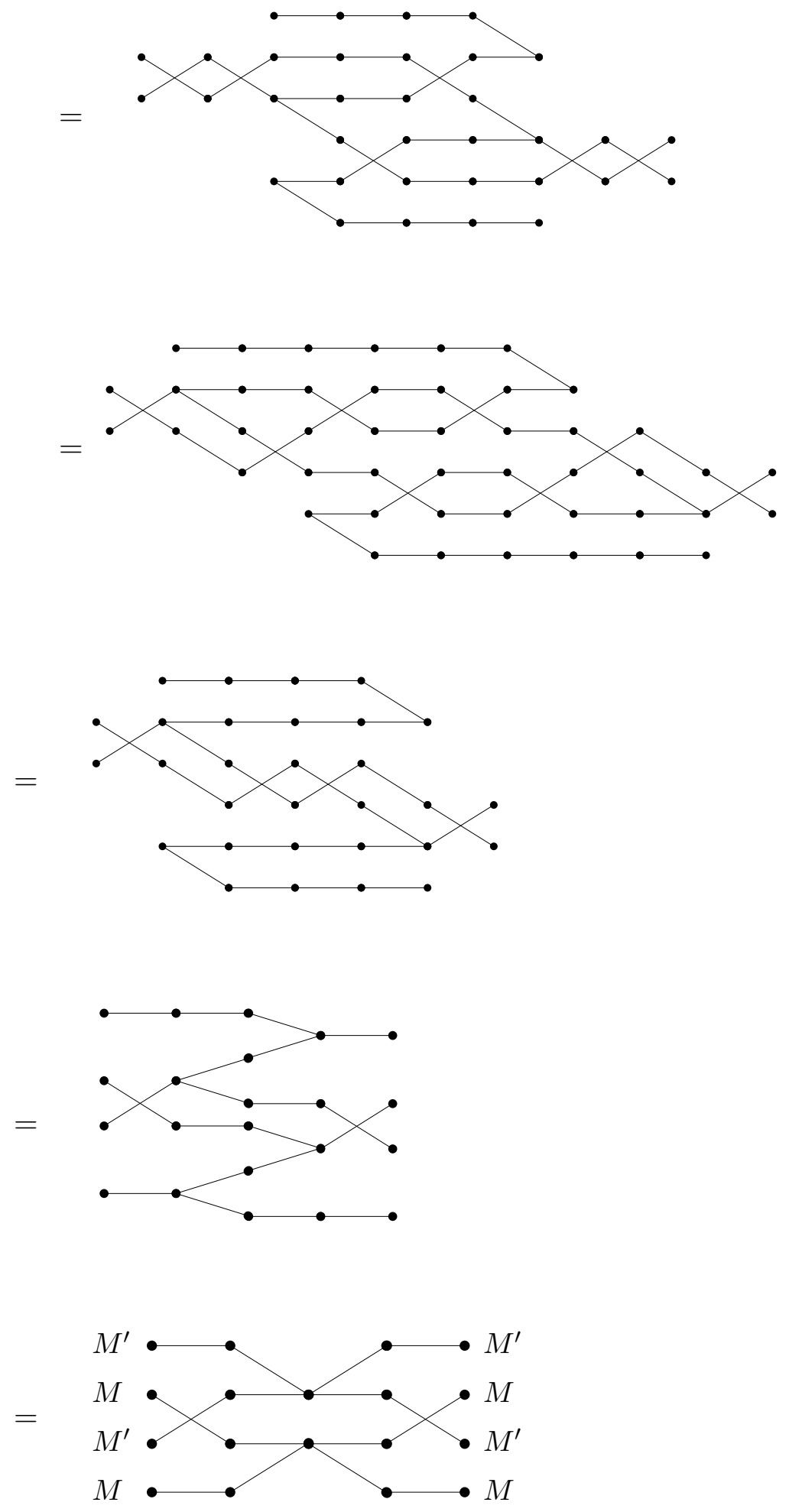


\subsubsection{The category $\mathrm{X}$ and an equivalence of categories}

Before constructing the category $\mathbf{X}$, we will first look at more generally how to generate a category from a directed graph $G$, following the method of [10]. This generated category is denoted $C(G)$. Next, we consider the category $C(G)$ under some relation $R$ on $G$, and see how to construct a "quotient" category, $C(G) / R$, under this relation. This will be useful in the construction of $\mathbf{X}$.

Consider a directed graph $G=(A, O)$, where $O$ is the collection of vertices of $G$, and $A$ is the collection of edges of $G$. Since the graph is directed, one can define two functions: a domain function, $\partial_{0}$, which assigns to each edge the vertex at the tail of that edge, and a co-domain function, $\partial_{1}$ which assigns to each edge the vertex at the tip of that edge.

$$
\partial_{0}: A \rightarrow O \quad \partial_{1}: A \rightarrow O
$$

The objects of the category $C(G)$ (constructed from the graph $G=(A, O)$ ) are exactly the vertices of $G$, the collection $O$. Every morphism of $C(G)$ is either an identity morphism on some element of $O$, or is a composition of the edges of $A$. That is, for objects $a, b$ in $C(G)$, there is a morphism $f \in C(a, b)$ (a morphism $a \rightarrow b$ in $\operatorname{Mor} C$ ) if and only if there is a directed path from $a$ to $b$ in $G$, or $f$ is the identity morphism.

We now can generate a category from a directed graph $G$.

Next we describe how it is possible to construct a category under some relations, $R$. Consider all the morphisms from $a$ to $b$ in a category $C$, denoted $C(a, b)$. Let $R_{a, b}$ be a relation on this set. Let $C / R$ be the quotient category which is defined in the image of a functor $Q$. We define $Q=Q_{R}$ to be the functor which satisfies the following two properties.

1. If $f R_{a, b} f^{\prime}$, then $Q f=Q f^{\prime}$ for every $f, f^{\prime} \in C(a, b)$. 
2. If $H: C \rightarrow D$ is any functor such that $H f=H f^{\prime}$ whenever $f R_{a, b} f^{\prime}$, then there is a unique functor $H^{\prime}: C / R \rightarrow D$ such that $H^{\prime} \circ Q_{R}=H$.

That is, the functor $Q$ is the identity on the objects of $C$, and sends each $f \in C(a, b)$ to its equivalence class with respect to an equivalence relation $R_{a, b}^{\prime}$ extending $R_{a, b}$. Therefore, the hom-sets $C / R(a, b)$ in $C / R$ are the equivalence classes of $C(a, b)$ in $C$ under the relation $R_{a, b}^{\prime}$. Now consider $R$ to be a function from any pair of objects $(a, b) \in \mathrm{ObC} \times \mathrm{ObC}$.

In other words, $Q$ is the universal functor on the category $C$ with $Q f=Q f^{\prime}$ whenever $f R f^{\prime}$. We will briefly describe the idea of the proof.

We will call $S$ a congruence relation if $(i)$ for every $a, b \in \mathrm{ObC}, S_{a, b}$ is an equivalence relation on $C(a, b)$, and ( $i i)$ for all maps $g \in C\left(a^{\prime}, a\right)$ and $h \in C\left(b, b^{\prime}\right)$, if $f \sim_{S_{a, b}} f^{\prime}$, then $h f g \sim_{S_{a^{\prime}, b^{\prime}}} h f^{\prime} g$. For every relation $R$ as above, there exists a least congruence, $R^{\prime}$ extending $R$. That is, $R^{\prime}$ is a congruence relation and $R \subseteq R^{\prime}$, and if $R \subseteq S$, then $R^{\prime} \subseteq S$.

The category $C(G) / R$ is now what we define to be the category generated by $G$ with relations $R$. This provides us exactly with the tools we need in the next section to define a new category, $\mathbf{X}$.

We now alter our definition of generate again. Suppose we let our final category have a monoidal product as well. That is, there is a monoidal product on objects and a neutral object. Instead of using the word "generate" to only mean a category $C(G)$ where morphisms are compositions of morphisms of a graph, we now mean to take a larger category $C_{m}(G)$ where the morphisms are also combinations under the monoidal product. For this category to be well defined under taking relations, we need some extra conditions, which we will talk about soon. 


\section{The category $\mathrm{X}$}

We now define a symmetric strict monoidal category called $(\mathbf{X},+, \mathbf{0}, t)$ as follows. The objects of $\mathbf{X}$ are generated by $\mathbf{1}$. That is, every object is of the form

$$
\mathbf{n}:=\underbrace{1+1+\cdots+1}_{n}
$$

for $n \in \mathbb{N}$, and there is a neutral object $\mathbf{0}$.

All morphisms are generated by multiplication $\left(\mu_{\mathbf{X}}\right)$, co-multiplication $\left(\delta_{\mathbf{X}}\right)$, unit $\left(\eta_{\mathbf{X}}\right)$, co-unit $\left(\epsilon_{\mathbf{X}}\right)$, the twist maps $t$, and of course the identity map on $\mathbf{1}\left(\operatorname{id}_{\mathbf{1}}\right)$.

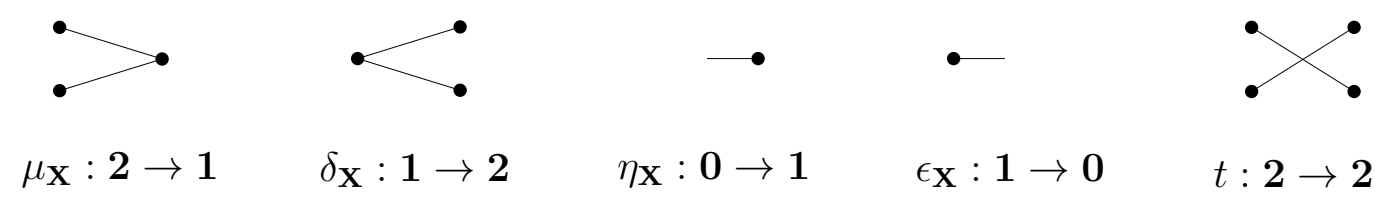

We now draw below a graph that generates $\mathbf{X}$, which, to be brief, are just compositions of (monoidal) products of these maps. A description of its monoidal product follows.

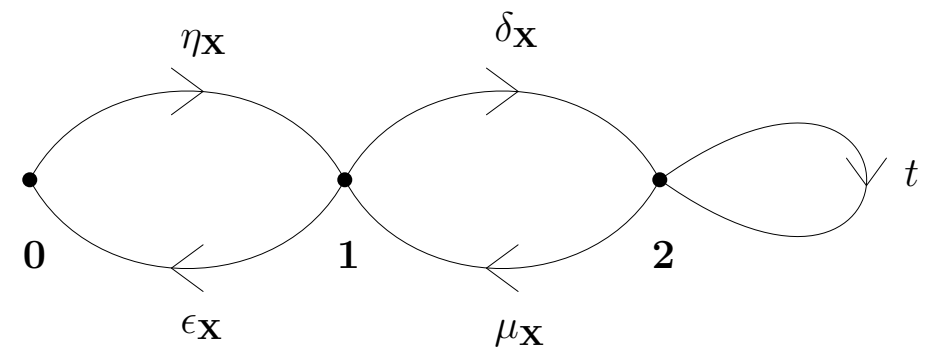

We now know that the compositions in the graph above are morphisms in our category. Now we discuss the monoidal product. Every morphism in $\mathbf{X}$ is the canonical sum of morphisms $\mu_{\mathbf{X}}, \delta_{\mathbf{X}}, \eta_{\mathbf{X}}, \epsilon_{\mathbf{X}}, t$, and $\mathrm{id}_{\mathbf{1}}$. So, a cardinal sum of morphisms $f_{1}+f_{2}+\cdots+f_{k}$ will be a morphism with domain $\operatorname{dom} f_{1}+\operatorname{dom} f_{2}+\cdots+\operatorname{dom} f_{k}$ and 
co-domain $\operatorname{cod} f_{1}+\operatorname{cod} f_{2}+\cdots+\operatorname{cod} f_{k}$.

This collection of cardinal sums of $\mu_{\mathbf{X}}, \delta_{\mathbf{X}}, \eta_{\mathbf{X}}, \epsilon_{\mathbf{X}}, t$, and $\mathrm{id}_{\mathbf{1}}$ does not complete the collection of morphisms, rather it generates it with composites, as described in the previous section by generating categories from graphs. Therefore, we know that this forms a category.

Next, we want to describe the relations on this category, and define the category $\mathbf{X}$ to be the quotient with respect to these relations. The relations are the unit, co-unit, and Frobenius relations.

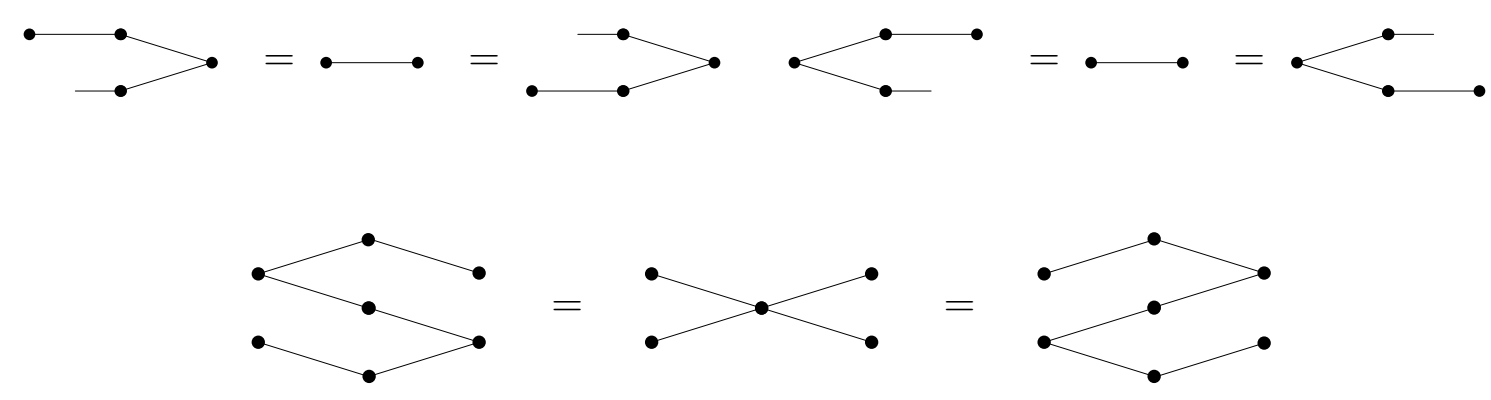

These equivalences extend to all cardinal sums. That is, if $h: \mathbf{k} \rightarrow \mathbf{l}$ and $g: \mathbf{k}^{\prime} \rightarrow \mathbf{l}^{\prime}$ are morphisms in $\mathbf{X}$, if $f \sim_{R_{\mathbf{m}, \mathbf{n}}} f^{\prime}$, then $h+f+g \sim_{R_{\mathbf{k}+\mathbf{m}+\mathbf{k}^{\prime}, \mathbf{1}+\mathbf{n}+\mathbf{1}^{\prime}}} h+f^{\prime}+g$. Also, recall that since we want $\mathbf{X}$ to be monoidal, we require $h+g=\left(\operatorname{id}_{\mathbf{l}}+g\right)\left(h+\mathrm{id}_{k^{\prime}}\right)=$ $\left(h+\operatorname{id}_{\mathbf{l}^{\prime}}\right)\left(\operatorname{id}_{k}+g\right)$.

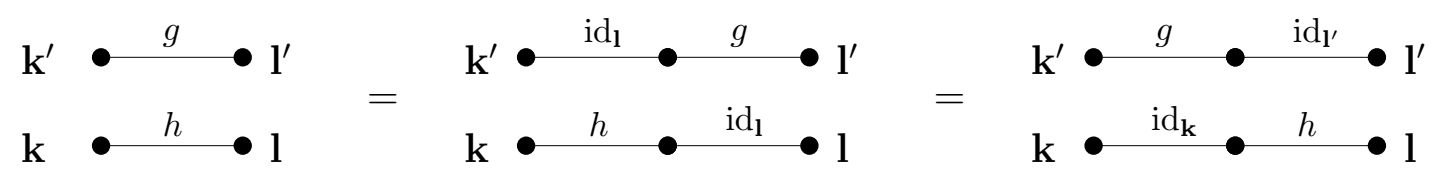

Lastly, we want $t$ to be a twist map, as defined in Definition . Also, we require that $\mu_{\mathbf{X}}$ is commutative and $\delta_{\mathbf{X}}$ is co-commutative.

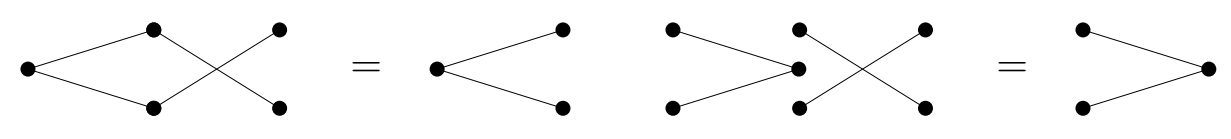


These are all the relations we require. Hence we have defined the category $\mathbf{X}$. Note that since $\mathbf{X}$ is a symmetric monoidal category under these relations, every object in $\mathbf{X}$ is a commutative Frobenius object. Hence, these relations imply associativity of $\mu_{\mathbf{X}}$, etc., as we have shown in previous sections.

Theorem 6.2.8. For a symmetric monoidal category $(\mathbf{V}, \square, I, \tau)$ and the category $\mathbf{X}$, we have the following isomorphism of categories.

$$
\operatorname{SymMonCat}(\mathrm{X}, \mathrm{V}) \cong \operatorname{cFrob}(\mathrm{V})
$$

Proof. We have shown $\operatorname{SymMonCat}(\Phi, \mathbf{V}) \cong \mathbf{c M o n}(\mathbf{V})$ in Theorem 6.1.10. This extends to the dual, that $\operatorname{SymMonCat}\left(\Phi^{\text {op }}, \mathbf{V}\right) \cong \mathbf{c} \operatorname{Comon}(\mathbf{V})$. For this proof, it is sufficient to discuss how the theorems extend to $\operatorname{SymMonCat}(\mathbf{X}, \mathbf{V}) \cong \mathbf{c F r o b}(\mathbf{V})$. In fact, much of the work is already done, and we use a similar pair of functors $\mathcal{M}, \mathcal{N}$ as in 6.1.10. The only things we have yet to consider is the Frobenius relation in $\mathbf{X}$, and the Frobenius relation of the objects of $\mathbf{c F r o b V}$.

We show this isomorphism of categories by defining a similar pair of functors $\mathcal{M}, \mathcal{N}$ as in Theorem 6.1.10. Specifically, we check that $\mathcal{M}, \mathcal{N}$ are well defined functors and that $\mathcal{M} \circ \mathcal{N}=\operatorname{id}_{\mathbf{c M o n}(\mathbf{V})}$ and $\mathcal{N} \circ \mathcal{M}=\operatorname{id}_{\operatorname{SymMonCat}(\Phi, \mathbf{V})}$.

$$
\begin{array}{rlr}
\mathcal{M}: \operatorname{SymMonCat}(\mathbf{X}, \mathbf{V}) & \rightarrow & \operatorname{cFrob}(\mathbf{V}) \\
{[F: \mathbf{X} \rightarrow \mathbf{V}]} & \mapsto & \left(F \mathbf{1}, F\left(\mu_{\mathbf{X}}\right), F\left(\delta_{\mathbf{X}}\right), F\left(\eta_{\mathbf{X}}\right), F\left(\epsilon_{\mathbf{X}}\right), F(t)\right) \\
u: F \rightarrow G & \mapsto & \left(u_{\mathbf{1}}: F \mathbf{1} \rightarrow G \mathbf{1}\right)
\end{array}
$$

We know from Theorem 6.1 .10 and its dual that $F(\mathbf{1})$ is a commutative monoid and co-monoid, and therefore satisifies the unit and co-unit relations. We only need to check $F(\mathbf{1})$ satisfies the Frobenius relation. This holds by the monoidal functorality of $F$, since $F\left(\left(\mu_{\mathbf{X}}+\mathrm{id}\right) \circ\left(\mathrm{id}+\delta_{\mathbf{X}}\right)\right)=\left(F \mu_{\mathbf{X}}+\mathrm{id}_{F \mathbf{1}}\right) \circ\left(\mathrm{id}_{F \mathbf{1}}+F \delta_{\mathbf{X}}\right)$. So since the Frobenius relation holds with multiplication and co-multiplication $\mu_{\mathbf{X}}$ and $\delta_{\mathbf{X}}$ in $\mathbf{X}$, the Frobe- 
nius relation on object $F \mathbf{1}$ holds with multiplication $F \mu_{\mathbf{X}}$ and co-multiplication $F \delta_{\mathbf{X}}$ in $\mathbf{V}$.

To show $F$ is well defined on morphisms, we need to show $u_{\mathbf{1}}$ is a Frobenius homomorphism. This is already done for us by the proof of Theorem 6.1.10 and its dual, since from these we know $u_{1}$ is a monoid and co-monoid homomorphism, which is all we require to say $u_{1}$ is a Frobenius homomorphism.

$$
\begin{aligned}
& \mathcal{N}: \quad \operatorname{cFrob}(\mathbf{V}) \rightarrow \operatorname{SymMonCat}(\mathbf{X}, \mathbf{V}) \\
& \left(\begin{array}{c}
M \\
\mu \\
\delta \\
\eta \\
\epsilon \\
\tau
\end{array}\right) \quad \mapsto\left(\begin{array}{rlll}
1 & \mapsto & M \\
\mu_{\mathbf{X}} & \mapsto & \mu \\
F: & \delta_{\mathbf{X}} & \mapsto & \delta \\
\eta_{\mathbf{X}} & \mapsto & \eta \\
\epsilon_{\mathbf{X}} & \mapsto & \epsilon \\
t & \mapsto & \tau
\end{array}\right) \\
& \left(\psi: M \rightarrow M^{\prime}\right) \mapsto \quad\left(\begin{array}{c}
u: F \rightarrow F^{\prime} \\
\text { where } u_{1}:=\psi
\end{array}\right)
\end{aligned}
$$

For $(M, \mu, \delta, \eta, \epsilon, \tau)$ to be a commutative Frobenius object, we require that $\mu, \delta, \eta, \epsilon, \tau$ satisfy the unit, co-unit, and Frobenius relations. Since $\mathbf{X}$ only satisfies these minimum requirements, and the category is the universal category with respect to these relations, the sub-category generated by $\mu, \delta, \eta, \epsilon, \tau$ in $\mathbf{V}$ under the same (respective) relations must also have the same minimum congruences. Hence, the monoidal functor $F$ as defined above will be well defined. That $\left(u: F \rightarrow F^{\prime}\right)$ is a well defined natural transformation follows from Theorem 6.1.10.

It only remains to show that the two functors $\mathcal{M}$ and $\mathcal{N}$ are mutual inverses. Firstly, $\mathcal{M N}=\mathrm{id}_{\mathbf{c F r o b}(\mathbf{V})}$ as 


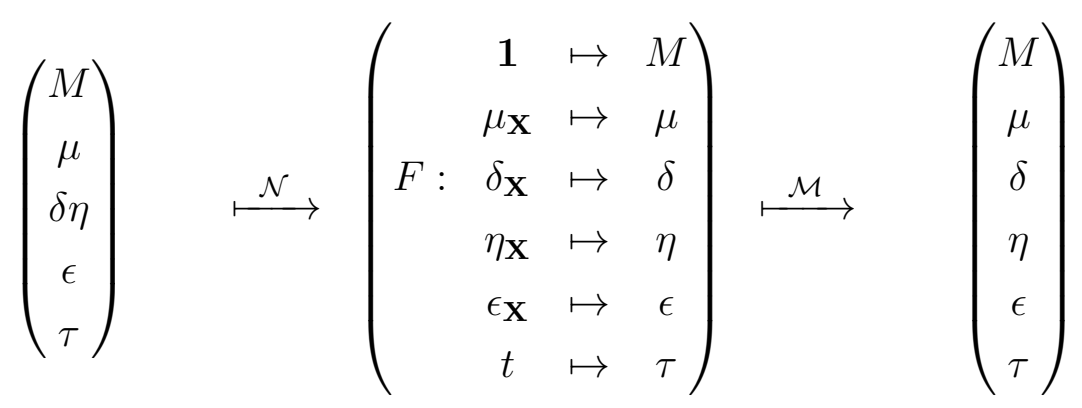

$$
\begin{aligned}
& \left(\psi: M \rightarrow M^{\prime}\right) \stackrel{\mathcal{N}}{\longmapsto}\left(\begin{array}{c}
u: F \rightarrow F^{\prime} \\
\text { where } u_{\mathbf{1}}=\psi
\end{array}\right) \stackrel{\mathcal{M}}{\longmapsto}\left(\psi: M \rightarrow M^{\prime}\right)
\end{aligned}
$$

$\mathcal{N M}=\operatorname{id}_{\text {SymMonCat }(\mathbf{X}, \mathbf{V})}$.

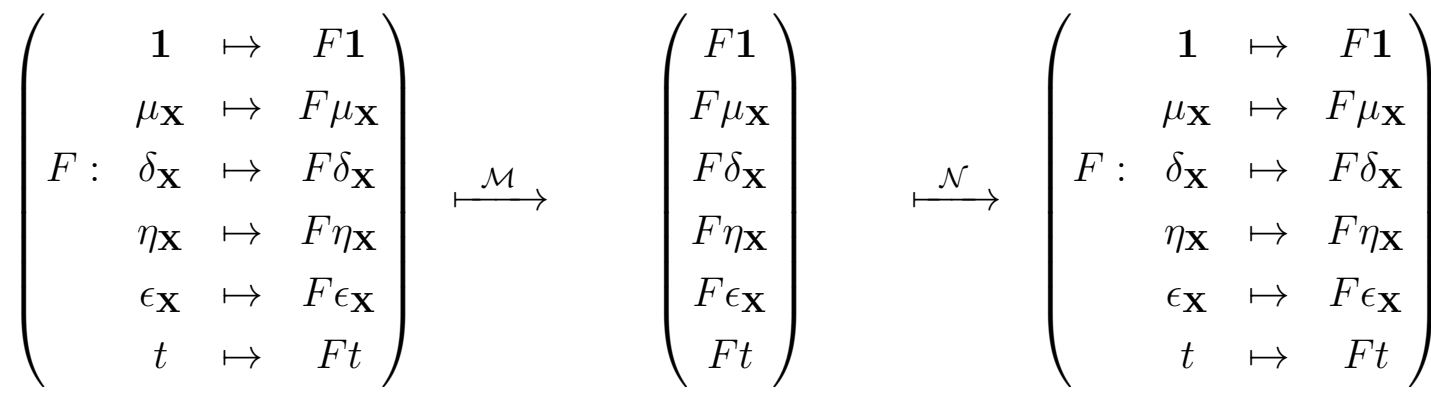

$$
\begin{aligned}
& \left(u: F \rightarrow F^{\prime}\right) \quad \stackrel{\mathcal{M}}{\longmapsto}\left(u_{1}: F \mathbf{1} \rightarrow F^{\prime} \mathbf{1}\right) \stackrel{\mathcal{N}}{\longmapsto} \quad\left(u: F \rightarrow F^{\prime}\right)
\end{aligned}
$$

as required.

\subsection{Cobordisms}

The next section describes another category called $2 \mathrm{Cob}$. The aim is to show that the skeleton of $2 \mathbf{C o b}$ (which we abusively also call $2 \mathbf{C o b}$ ) is isomorphic to the category $\mathbf{X}$ described in the previous section. Instead of sticklike figures, the morphisms will be replaced by surfaces, where two morphisms are equivalent if and only if they are equivalent topologically (specifically, equivalent under orientation preserving dif- 
feomorphism).

We begin with some background in differential topology. This section is a stand alone section that we will not refer to once we have proven what we need - how to construct the category $2 \mathbf{C o b}$, the generators and relations of $2 \mathbf{C o b}$, and an isomorphism of categories. For no reason other than it required no extra effort, we have defined the category $n$ Cob for all $n$, though all we will need is the 2-dimensional case.

We work mostly with compact oriented 2-dimensional manifolds and oriented 1dimensional manifolds without boundary. Our manifolds will not be embedded in any higher dimensional space, but rather are abstract topological spaces.

\subsubsection{Some background in differential topology}

\section{Differentiable manifolds}

Let $\lambda: \mathbb{R}^{n} \rightarrow \mathbb{R}$ be a non-zero linear transformation. Recall a $h a l f$-space, $H^{n}$, is a subspace of $\mathbb{R}^{n}$ such that

$$
H^{n}:=\left\{x \in \mathbb{R}^{n}: \lambda x \geq 0\right\}
$$

with the topology inherited from $\mathbb{R}^{n}$. The boundary of $H^{n}$, denoted $\partial H^{n}$, is the same as the topological boundary as a subspace of $\mathbb{R}^{n}$, and consists of the points in the subset $\left\{x \in \mathbb{R}^{n}: \lambda x=0\right\}$ of $H^{n}$.

A real $n$-dimensional manifold is a metrisable topological space that locally resembles $n$-dimensional Euclidean space. More precisely, a metrisable topological space $M$ is called a manifold if there is an open cover $\left\{U_{i}\right\}_{i \in \Lambda}$ of $M$, such that for every $i \in \Lambda$, there is a homeomorphism to an open subset of a half-space $H^{n}$, $\Phi_{i}: U_{i} \rightarrow \Phi_{i}\left(U_{i}\right) \subset H^{n}$. The open cover $\left\{U_{i}\right\}_{i \in \Lambda}$ is called an atlas of $M$, and the homeomorphisms $\Phi_{i}$ are called the charts of $M$. We sometimes refer to an real $n$ dimensional manifold as an $n$-manifold. 
The boundary points of $M$ are the points which are mapped to $\partial H^{n}$ in the charts, and together, the boundary of $M$ is denoted $\partial M$. A closed manifold will be a compact manifold without boundary (this is not standard language).

A sub-manifold of a manifold $M$ is a subset of $M$ which is itself a manifold with the inherited topology from $M$. We will consider the empty $n$-manifold, $\varnothing_{n}$, to be a manifold for each $n$. We will not require our manifolds to be connected.

The only manifolds we wish to consider are differentiable manifolds (also known as smooth manifolds). These are manifolds with charts that are $C^{\infty}$, or infinitely differentiable (alternatively known as smooth) at every point of the manifold. A map $f$ between differentiable manifolds is called a diffeomorphism if $f$ is a homeomorphism and both $f$ and $f^{-1}$ are infinitely differentiable.

Suppose $M, M^{\prime}$ are $n$-dimensional manifolds. The disjoint union of $M$ and $M^{\prime}$, denoted $M \amalg M^{\prime}$, is a $n$-dimensional manifold in which one can embed both $M$ and $M^{\prime}$. The disjoint union of $M$ and $M^{\prime}$ is defined to be the disjoint union of the underlying sets of $M$ and $M^{\prime}$, and the collection of charts is the union of all the charts of both $M$ and $M^{\prime}$.

Again, suppose $M, M^{\prime}$ are $n$-dimensional manifolds, and let $A$ be a subspace of $M$ with an embedding $f: A \hookrightarrow M^{\prime}$. One can define a new topological space by taking the disjoint union of $M$ and $M^{\prime}$, and then associating each element $a \in A \subset M$ with $f(a) \in M^{\prime}$. This is called the adjunction space of $M$ and $M^{\prime}$ over $f(A)$, and is denoted $M \amalg_{f} M^{\prime}$. In the case $A$ is a closed $(n-1)$-dimensional manifold which is a subset of the boundary of $M$, and $f(A)$ is a subset of the boundary of $M^{\prime}$, then $M \amalg_{f} M$ is itself a manifold. As an abuse of notation, if $\Sigma$ is a closed $(n-1)$-dimensional manifold and is a subset of the boundaries of $M$ and $M^{\prime}$, we will write $M \amalg_{\Sigma} M^{\prime}$ as the adjunction space of $M$ and $M^{\prime}$ where $\Sigma$ is associated. 


\section{Orientable manifolds}

In describing orientable manifolds, we are intentionally brief, as a full description is outside the scope of this thesis, and we refer the reader to [5]. In particular, we have not defined a tangent space of a manifold.

We begin by defining orientability on vector spaces and then extend this to how to define an orientation on a manifold. This uses the collection of all tangent spaces of a manifold.

Consider a vector space $V$. We first define a relation on the collection of all ordered bases of $V$. For ordered bases $B, B^{\prime}$ of $V$, we let $B \sim_{R} B^{\prime}$ if there exists a matrix with a positive determinant that maps one to the other. Hence, there are exactly two equivalence classes under this relation, which once we assign a basis to be a "positive basis," each basis can be referred to as either a positive basis or a negative basis. An orientation of a vector space $V$ is an equivalence class of the ordered bases of $V$. Note that there are exactly two orientations of $V$.

An orientation of a manifold is an orientation of the tangent space at each point $x \in M$. such that there is a smooth change of the tangent space to any other point in the manifold such that the orientation is equivalent.

We will only be considering oriented manifolds, which are manifolds for which there exists an orientation. For an oriented manifold $M$, the manifold with the opposite orientation is written with an over-bar: $\bar{M}$.

Let $T_{x} M$ be the tangent space of a manifold $M$ at $x$. Consider now an oriented manifold $M$ of dimension $n$ with a closed sub-manifold $\Sigma$ of dimension $n-1$ with its own orientation. Let $\left[v_{1}, v_{2}, \ldots, v_{n-1}\right]$ be a positive basis for the tangent space of $x \in \Sigma, T_{x} \Sigma$. Consider a vector $w \in T_{x} M$ not contained in $T_{x} \Sigma$. Then $w$ is called a positive normal if $\left[v_{1}, v_{2}, \ldots, v_{n-1}, w\right]$ is a positive basis of $T_{x} M$.

Now, consider the case where $\Sigma$ is a connected component of the boundary of $M$ 
with an orientation (in other words, $\Sigma$ is a closed manifold contained in $\partial M$ ). Then if the positive normal points inwards towards $M$ - that is toward $H^{n} \backslash \partial H^{n}$ - then $\Sigma$ is called an in-boundary. Otherwise, it is called an out-boundary. The in-boundary (respectively out-boundary) of $M$ is the collection of all in-boundaries (respectively out-boundary) of $M$.

Let $M, M^{\prime}$ be oriented $n$-dimensional manifolds. A map $f: M \rightarrow M^{\prime}$ is an orientation preserving map if the induced maps on the tangent space brings a positively oriented basis to a positively oriented basis.

Note that a manifold as we have defined it is not embedded in any higher dimensional space. Hence, there is no concept of part of a manifold passing over or under another part of a manifold.

\section{Cobordisms}

Consider two closed $(n-1)$-manifolds $\Sigma_{1}$ and $\Sigma_{2}$. An $n$-cobordism $M$ from $\Sigma_{1}$ to $\Sigma_{2}$ is an $n$-manifold with the boundary $\partial M=\Sigma_{1} \amalg \Sigma_{2}$. A cobordism from $\Sigma_{1}$ to $\Sigma_{2}$ is denoted by the arrow $M: \Sigma_{1} \rightsquigarrow \Sigma_{2}$ (though at this stage there is no distinction between $\Sigma_{1}$ and $\left.\Sigma_{2}\right)$. Two closed $(n-1)$-manifolds are called cobordant if there exists a cobordism between them. Cobordance forms an equivalence relation on differentiable manifolds.

An oriented cobordism is a cobordism of oriented manifolds. Consider oriented $(n-1)$ manifolds $\Sigma_{1}$ and $\Sigma_{2}$. We want to formalise a concept of an oriented cobordism $M$ from $\Sigma_{1}$ to $\Sigma_{2}$, which we do by making $\Sigma_{1}$ the in-boundary of $M$, and $\Sigma_{2}$ the outboundary of $M$. More precisely, an oriented cobordism from $\Sigma_{1}$ to $\Sigma_{2}$ will be defined as a manifold $M$ such that the embedding of $\Sigma_{1}$ is (orientation preserving) diffeomorphic to the in-boundaries of $M$, and the embedding of $\Sigma_{2}$ is (orientation preserving) diffeomorphic to the out-boundaries of $M$. 


$$
\Sigma_{1} \stackrel{\phi_{1}}{\longleftrightarrow} M \stackrel{\phi_{2}}{\longleftrightarrow} \Sigma_{2}
$$

\section{Definition 6.3.1. Equivalent cobordisms}

Two cobordisms $M, M^{\prime}: \Sigma_{1} \rightsquigarrow \Sigma_{2}$ are called equivalent if there is an orientation preserving diffeomorphism $M \rightarrow M^{\prime}$ such that the following diagram commutes. Note that this means $\Sigma_{1}$ and $\Sigma_{2}$ are fixed on $M$ and $M^{\prime}$, and a diffeomorphism between $M$ and $M^{\prime}$ must respect these boundaries.

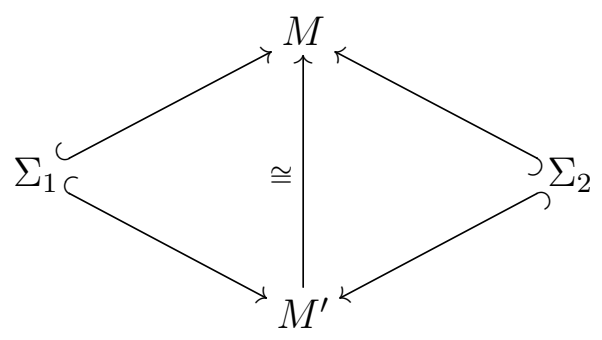

The boundary of an oriented cobordism $M$ is the disjoint union of closed in-boundaries and out-boundaries of $M$. In the case that both the in-boundary and the outboundary are empty manifolds, the cobordism is a closed manifold. For example, an $n$-sphere is an oriented $n$-cobordism where both $\Sigma_{1}$ and $\Sigma_{2}$ are the empty manifold, $\varnothing_{n-1}$.
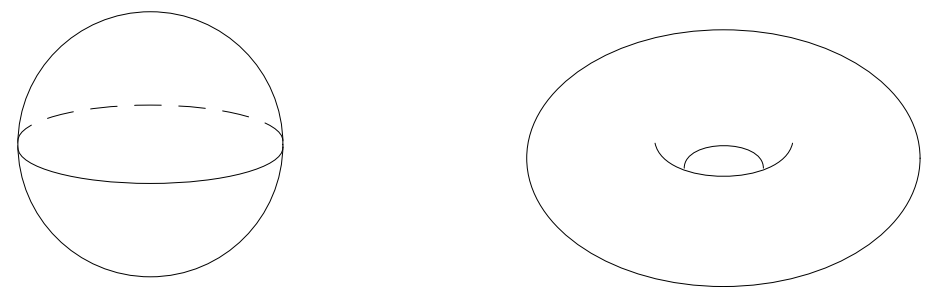
Next, we will describe a category $n \mathbf{C o b}$, which has oriented $(n-1)$-dimensional manifolds as objects. Unlike more standard categories, the morphisms of this category are not functions between sets or functions with additional structure. Instead, the collection of oriented $n$-dimensional cobordisms form the morphisms of a category under an appropriate equivalence relation. Also, since we are considering oriented cobordisms, there are exactly two types of boundaries. Hence, the boundary of a cobordism can be divided into what will be defined as either the domain or the codomain of the morphism.

More formally, the objects of the category $n \mathbf{C o b}$ are the equivalence classes of oriented, compact, differentiable $(n-1)$-manifolds under orientation preserving diffeomorphism. The morphisms of $n \mathbf{C o b}$ are the equivalence classes of oriented $n$ cobordisms (as in Definition 6.3.1), where the in-boundaries are the domain, and the out-boundaries are the co-domain.

We claim that $n$ Cob is indeed a category for each $n$. Firstly, we introduce an identity cobordism. The identity morphism of an $(n-1)$-manifold $\Sigma$ is simply the cylinder $\Sigma \times I$, where $I$ is the closed unit interval. The embedding $\phi_{0}: \Sigma \hookrightarrow \Sigma \times I: x \mapsto(x, 0)$ maps $\Sigma$ diffeomorphically onto the in-boundary $\Sigma \times\{0\}$, and $\phi_{1}: \Sigma \hookrightarrow \Sigma \times I: x \mapsto$ $(x, 1)$ maps $\Sigma$ diffeomorphically onto the out-boundary $\Sigma \times\{1\}$, as required.

We will make a brief note about the orientation of the product $(\Sigma \times I)$. Let $\left[v_{1}, \ldots, v_{n-1}\right]$ be a basis for $T_{x} \Sigma$, and $[w]$ be a basis of $T_{y} I$, which is in the direction of $1 \in I$. The orientation of $(\Sigma \times I)$ is defined to have as a positive basis $\left[v_{1}, \ldots, v_{n-1}, w\right]$. Hence, the embedding $\phi_{0}$ does map $\Sigma$ onto the in-boundary, and $\phi_{1}$ maps $\Sigma$ onto the outboundary, and are indeed orientation preserving maps.

The composition of two cobordisms $M: \Sigma_{0} \rightsquigarrow \Sigma_{1}$ and $M^{\prime}: \Sigma_{1} \rightsquigarrow \Sigma_{2}$ is the cobor$\operatorname{dism} M \amalg_{\Sigma_{1}} M^{\prime}: \Sigma_{0} \rightsquigarrow \Sigma_{2}$, that is a new cobordism formed by gluing $M$ and $M^{\prime}$ at $\Sigma_{1}$ (consistently with its orientation). Not every gluing of this type is necessarily a differentiable manifold, but because the morphisms of $n$ Cob are equivalence classes, 
we need only to know that a differentiable manifold $M M^{\prime}$ exists. This is covered in detail in [7] .

The category $n$ Cob is a monoidal category. Disjoint union forms the monoidal product, and the neutral object is the empty manifold, $\varnothing_{n-1}$. Note that unlike in the category of topological spaces, disjoint union does not form a co-product. Also, there is no initial object.

\subsubsection{2-cobordisms}

For the rest of this section, when we refer to a cobordism we mean the equivalence class of all compact oriented 2-dimensional cobordisms under orientation preserving diffeomorphism with respect to the boundaries (see Definition 6.3.1).

Every closed connected 1-manifold is diffeomorphic to a circle. It follows that every closed 1-manifold (except the empty manifold $\varnothing_{1}$ ) is the disjoint union of circles. This means, under the operation of disjoint union, the objects of $2 \mathbf{C o b}$ are 'generated' by a circle under disjoint union. For any manifold $\Sigma$, the disjoint union $\Sigma \amalg \varnothing_{1}$ is $\Sigma$.

It is convenient to draw the in-boundaries of a cobordism on the left and the outboundaries on the right, and thus morphisms in $2 \mathbf{C o b}$ are drawn with the domain on the left and the co-domain on the right. For example, a 2-cobordism with inboundary $\Sigma$ and out-boundary $\Sigma$ will be drawn as follows. Here, the arrows denote the direction of the positive normal of the cobordism with respect to that boundary.

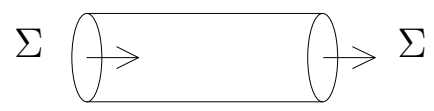


Hence, the two copies of $\Sigma$ above have different normals with respect to the cylinder, and one is an in-boundary and one is an out-boundary. Moreover, a 2-cobordism with in-boundary $\Sigma \amalg \bar{\Sigma}$ which is diffeomorphic to a cylinder could be drawn as on the left. Our convention will be to draw this cobordism like the right diagram. So, all positive normals with respect to the boundary of the manifold will point right.
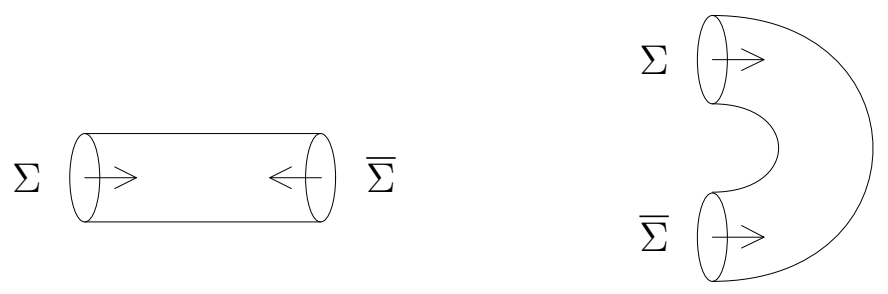

Two cobordisms that are (orientation preserving) diffeomorphic are not necessarily the same cobordism in $2 \mathbf{C o b}$ - one needs to consider the boundaries of cobordism. The simplest example of different diffeomorphic cobordisms in $2 \mathbf{C o b}$ is the twist cobordism and the identity map on two circles. Hence the ordering of the circles in the boundary (on a vertical as drawn below) are drawn with respect to an ordering, hence the drawing on the right will represent the identity cobordism on two circles.
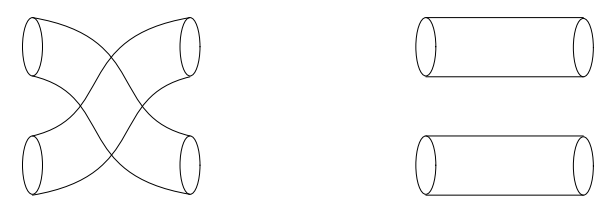

Consider all cobordisms from $m$ circles to $m$ circles which are diffeomorphic to $m$ disjoint cylinders. These can all be constructed from twist cobordisms and identity cobordisms, under composition and disjoint union of cobordisms. This collection has the structure of the symmetric group on $m$ elements, $S_{m}$, where the operation is composition of cobordisms. 
As an aside, we draw the twist cobordism with the two cylinders "intersecting" (on the page) to reinforce that our manifolds are not embedded in any higher dimensional space. Drawing one cylinder in front of the other can lead to the misconception that the following two cobordisms aren't the same.
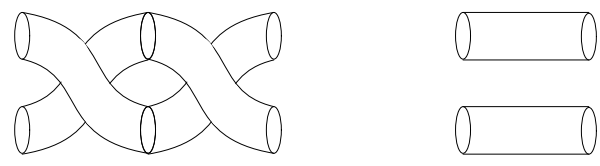

Theorem 6.3.2. Every cobordism in the category 2 Cob can be written as a combination of the following six symbols.
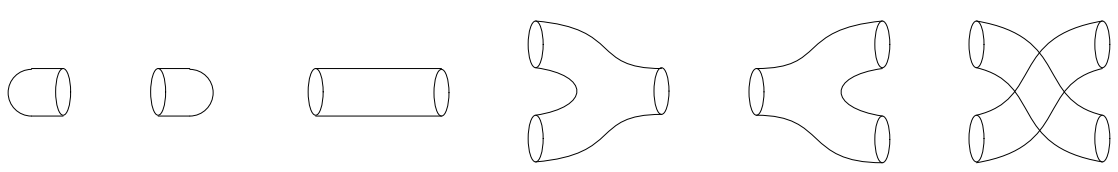

We now give a brief overview of how this theorem is proved.

Recall the genus of a connected compact orientable surface is the number of "2dimensional" holes in that surface. For example, a torus has genus 1, and a sphere has genus 0 . The genus of a surface with a boundary is the genus of the same surface with closed disks attached to each boundary. Hence, the genus is an invariant of the surface. Therefore, we can use this to say that every 2-cobordism can be rewritten in a normal form as follows.

Every connected oriented 2-cobordism is homeomorphic to a 'normal form' of a connected surface. That is, a connected surface with $m$ in-boundaries, $n$ out-boundaries, and of genus $g$. We will not prove this but describe how this can be seen. 

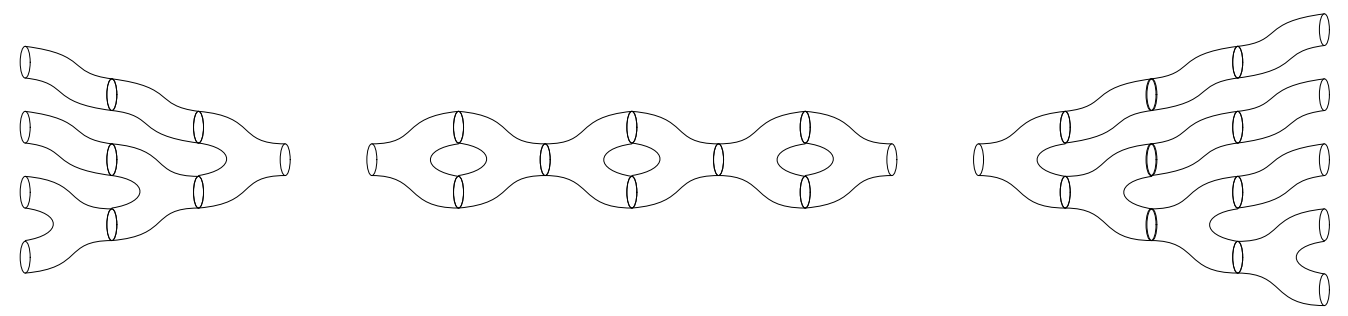

So, we now have shown that every connected 2-cobordism can be written as a combination of the following cobordisms, where the ends on the left are used for closed 2-cobordisms (cobordisms with no boundary).
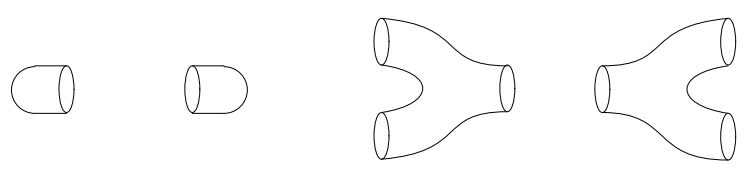

Next, we consider non-connected manifolds. Consider a 2-cobordism $M$ such that $M=M_{1} \amalg M_{2}$ for connected 2-cobordisms $M_{1}, M_{2}$. We know $M_{1}$ and $M_{2}$ can be written as a combination of the symbols $0,0,2,5$. The proof is not finished here though, as there is no reason to believe that $\operatorname{dom}(M)=\operatorname{dom}\left(M_{1}\right) \amalg \operatorname{dom}\left(M_{2}\right)$, and similarly with the co-domain. That is, it is not necessary that the in-boundaries of $M_{1}$ correspond to the first in-boundaries of $M$, and that the in-boundaries of $M_{2}$ correspond to the last in-boundaries of $M$. That is, although these manifolds will be diffeomorphic, they are not necessarily the same, in the same way the twist cobordism is not the same cobordism as the disjoint union of two "identity" cylinders.

Therefore, using the twist cobordisms, one can permute the in-boundaries of $M$ to the ordering $\operatorname{dom}\left(M_{1}\right) \amalg \operatorname{dom}\left(M_{2}\right)$ of the in-boundaries. Similarly, there is a collection of twist cobordisms that $\operatorname{map} \operatorname{cod}\left(M_{1}\right) \amalg \operatorname{cod}\left(M_{2}\right)$ to $\operatorname{cod}(M)$. Hence, pre-composing and post-composing $M_{1} \amalg M_{2}$ with the twist maps will leave us with our original cobordism, since the twist cobordisms will not change each of $M_{1}$ and $M_{2}$ as cobordism. Thus, we have shown that every 2-cobordism can be written as a composition of the symbols in Theorem 6.3.2, as required. 


\section{Relations in $2 \mathrm{Cob}$}

Next, we will examine some topological equivalences of composition of cobordisms, and thereby finding equivalent morphisms in the category 2 Cob. By doing so, we have essentially found the generators and relations of this category, in the language of Subsection 6.2.1. Consequently, this will give us the tools to prove that $\mathbf{X}$ and $2 \mathbf{C o b}$ are isomorphic as categories. What only will remain is to show that the following relations are sufficient.

The proofs of the following relations will not be done here, but we refer the reader to [7].

The disjoint union of cylinders is the identity in $2 \mathbf{C o b}$, as we can see below for each of the generators of the morphisms of $2 \mathrm{Cob}$.
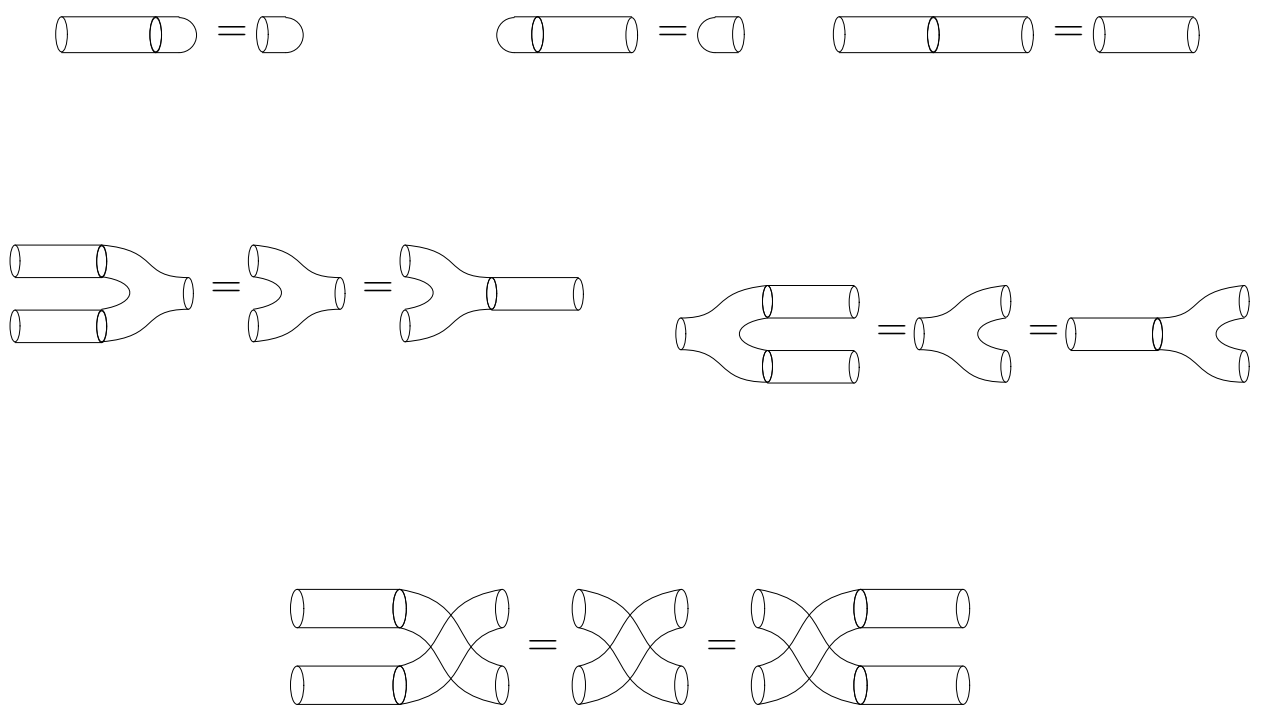

The above conditions are sufficient to say the disjoint union of cylinders is the identity morphism in $2 \mathbf{C o b}$. Consequently, we can forget about them as they are implied by 2 Cob being a category. Next, we show some other relations. 
Sewing in discs
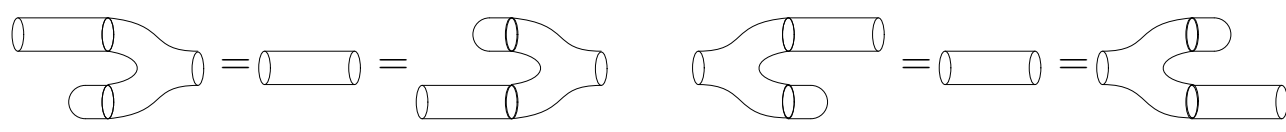

\section{Associativity and co-associativity}
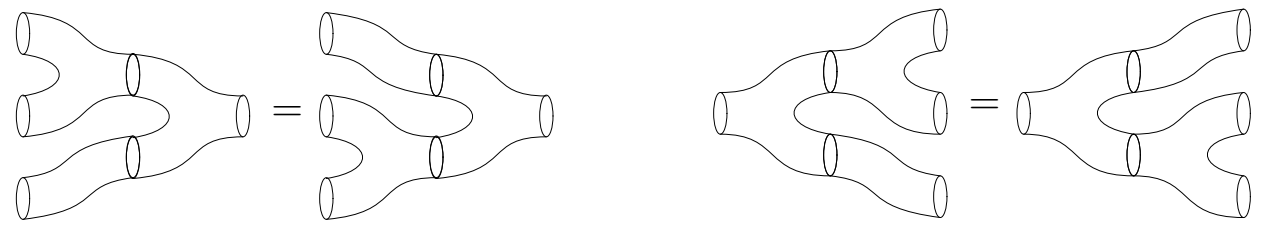

Commutativity and co-commutativity
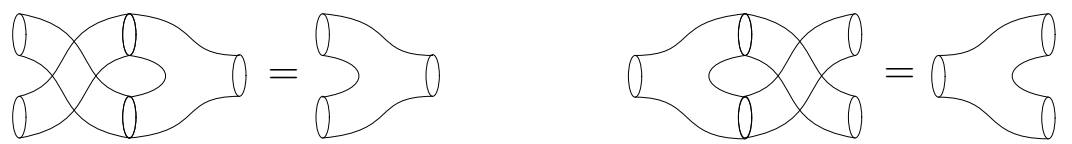

\section{Frobenius relation}

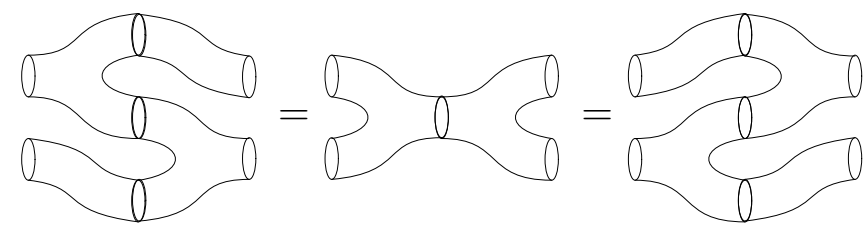

These relations are sufficient to define the category $2 \mathbf{C o b}$. In other words, there are no other relations in $2 \mathbf{C o b}$. We also will not prove this here, but it is done in [7].

Now, we know exactly what the morphisms and objects in the category of 2-cobordisms 
are. In other words, there is a complete classification of all the objects - the 1manifolds, and all the morphisms - the equivalence classes of 2-cobordisms.

Recall we defined the objects of the category $2 \mathrm{Cob}$ to be equivalence class of all closed 1-manifolds up to diffeomorphism, and morphisms of $2 \mathbf{C o b}$ to be equivalence class of all 2-cobordisms up to orientation preserving diffeomoprhism.

Proposition 6.3.3. $2 \mathrm{Cob}$ and $\mathrm{X}$ are isomorphic as categories.

Proof. First, we see that the objects of $2 \mathbf{C o b}$ are freely generated by a single connected 1-manifold under disjoint union. Also, there is a neutral object, the empty manifold $\varnothing_{1}$. Thus, there is a bijection between the objects.

Next, we need to show that $2 \mathbf{C o b}$ is generated by a disjoint union and composition of a multiplication map, co-multiplication map, unit, co-unit, twist map, and identity. We now assign cobordisms to each of these maps, as follows.

$$
\begin{aligned}
& F: \quad \mathrm{X} \quad \rightarrow \quad 2 \mathrm{Cob} \\
& \left(\begin{array}{cc}
1 & \\
\mu_{\mathbf{X}} & > \\
\delta_{\mathbf{X}} & < \\
\eta_{\mathbf{X}} & \cdots \\
\epsilon_{\mathbf{X}} & - \\
t & x
\end{array}\right) \mapsto\left(\begin{array}{c}
0 \\
\xi \\
\sigma \\
0 \\
8 \\
8
\end{array}\right)
\end{aligned}
$$

Hence, the functor $F$ assigns the upside-down pair of pants $\sum$ to the multiplication map, and so on. 
Recall from Subsection 6.2.1 that the category $\mathbf{X}$ is generated by five morphisms and the identity morphism under composition and the monoidal product + , and under four relations. These relations are the unit relation, the co-unit relation, the Frobenius relation, and the commutativity relation. These four relations were shown to hold in the previous subsection. Lastly, we need to show the sufficiency of these relations for $2 \mathrm{Cob}$. We also showed in Subsection 6.2.1 that these four relations imply associativity of multiplication, co-associativity of co-multiplication, commutativity and co-commutativity. The rest of the relations above (namely - the identity relations) are implied by $2 \mathbf{C o b}$ being a category. Hence, the collection of all relations of $\mathbf{X}$ and relations of $2 \mathbf{C o b}$ are the same under our assignment of the morphisms under $F$. Therefore, we have shown $F$ is a well defined functor which is fully faithful and bijective on objects, and hence is an isomorphism of categories.

\section{$6.4(1+1)-$ TQFTs}

To conclude this section, we put everything into the language of the title of this chapter. We have generalised everything to a general symmetric monoidal category, $(\mathbf{V}, \square, I, \tau)$.

Let the map $\sigma$ be the twist map $\sigma: V \otimes V \rightarrow V \otimes V$ which is defined by $\sigma(a \otimes b)=b \otimes a$, and let $T$ be the twist cobordism, $\mathbb{B}$.

\section{Definition 6.4.1. Topological Quantum Field Theories}

Let $K$-Vect be the category of vector spaces over a field $K$. A Topological Quantum Field Theory, or a $(n+1)-T Q F T$ is a symmetric monoidal functor from $(n \mathbf{C o b}, \amalg, \varnothing, T)$ to $(K$-Vect, $\otimes, K, \sigma)$. The collection of these functors form a category,

$$
(1+n)-\mathbf{T Q F T}:=\operatorname{SymMonCat}((n \operatorname{Cob}, \amalg, \varnothing, T),(K \text {-Vect, } \otimes, K, \sigma)) .
$$




\subsection{The Equivalence of categories}

The following theorem is a direct consequence of Theorem 6.2.8 and Proposition 6.3.3.

Theorem 6.5.1. For a symmetric monoidal category $(\mathbf{V}, \square, I, \tau)$, the following isomorphism of categories holds.

$$
\operatorname{SymMonHom}(2 \operatorname{Cob}, \mathrm{V}) \cong \operatorname{cFrob}(\mathrm{V})
$$

In application, we use the naturality of the isomorphism in Theorem 6.5.1, which is shown explicitly in Theorem 6.2.8. That is, for a commutative Frobenius object $(M, \mu, \delta, \eta, \epsilon, \tau)$ in a symmetric monoidal category $\mathbf{V}$, a morphism in $\mathbf{V}$ which can be written in terms of $\mu, \delta, \eta, \epsilon, \tau, \mathrm{id}_{M}$ under composition and the monoidal product can be represented by a cobordism. To show two morphisms in $\mathbf{V}$ of the form just described are the same in $\mathbf{V}$, it is sufficient to show that the corresponding cobordisms are orientation preserving diffeomorphic, or really homeomorphic with respect to their in-boundaries and out-boundaries.

Hence, when we refer to the TQFT applied to $M$, we mean the functor that sends $\sum$ to $\mu,\{\delta$ to $\delta$, and so on, as in the functor Theorem 6.2.8.

When we speak of cobordisms from now on, or rather draw diagram of them, we really mean the image of the cobordism in V. For example, the cobordism $\delta$ always represents $\delta$.

\subsection{Graded $R$-modules}

To put this in the context of this thesis, we have one remaining thing to do, which is show $\operatorname{gr}(R$-mod $)$, is a symmetric monoidal category. Then, once we show that 
our $V$ is a Frobenius object, we can apply a TQFT and replace all our graded linear maps with cobordisms! We will do so in the next chapter.

We now show that the category of $\mathbb{Z}$-graded $R$-modules, written $\operatorname{gr}(R$-mod $)$, is a symmetric monoidal category. To make things easier, we will use that $(R$-mod, $\otimes, R, \tau)$ is a symmetric monoidal category, as stated in [10, 7].

First, we define a neutral object, denoted $R_{0}$.

$$
\left(\operatorname{gr}(R-\bmod ), \otimes, R_{0}, \tau\right)
$$

With the neutral object and twist map defined as follows.

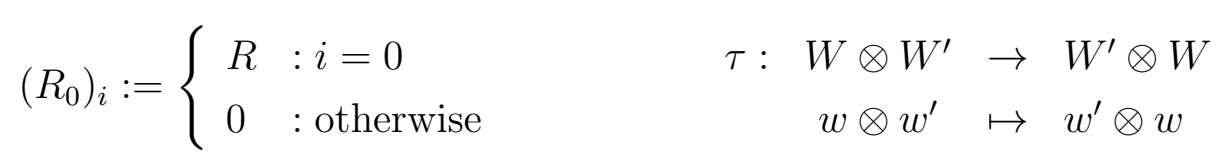

Let $U, W, X$ be $\mathbb{Z}$-graded $R$-modules. Next, we show that $(U \otimes W) \otimes X \cong U \otimes(W \otimes X)$.

$$
\begin{aligned}
\bigoplus_{i+j=k}\left(U_{i} \otimes(W \otimes X)_{j}\right) & =\bigoplus_{i+j=k}\left(U_{i} \otimes\left(\bigoplus_{l+m=j}\left(W_{l} \otimes X_{m}\right)\right)_{j}\right) \\
& =\bigoplus_{i+j=k}\left(\bigoplus_{l+m=j}\left(U_{i} \otimes\left(W_{l} \otimes X_{m}\right)\right)\right) \\
& =\bigoplus_{i+l+m=k}\left(U_{i} \otimes\left(W_{l} \otimes X_{m}\right)\right) \\
& \left.\cong \bigoplus_{i+l+m=k}\left(\left(U_{i} \otimes W_{l}\right) \otimes X_{m}\right)\right)
\end{aligned}
$$

The last line isomorphism is due to a natural isomorphism of $\left(U_{i} \otimes W_{l}\right) \otimes X_{m}$ and $U_{i} \otimes\left(W_{l} \otimes X_{m}\right)$. The last line is equal to $U \otimes(W \otimes X)$ by a symmetric argument to the one given above. 
Next, $R_{0} \otimes W \cong W \cong W \otimes R_{0}$.

$$
\begin{aligned}
\bigoplus_{i+j=k}\left(\left(R_{0}\right)_{i} \otimes W_{j}\right) & =\bigoplus_{j=k}\left(R \otimes W_{j}\right) \\
& =W
\end{aligned}
$$

Finally, we note that $\tau$ is an isomorphism, which is clear from the map above, and considering that every element can be written as a sum of elements from each $R$ module in the grading, on which $\tau$ is an isomorphism.

Therefore, we have shown that $\operatorname{gr}(R$-mod $)$ is a symmetric monoidal category, as required. 
168CHAPTER 6. TOPOLOGICAL QUANTUM FIELD THEORIES AND FROBENIUS OBJECTS 


\section{Chapter 7}

\section{TQFTs and the Khovanov homology}

We now have the tools to tie off some (rather important!) loose ends about the Khovanov homology we left in Chapter 4. We start by checking that the graded vector space $V$ is indeed a Frobenius object in the category of $\mathbb{Z}$-graded vector spaces. This involves first defining a unit and co-unit. Once this is done we apply a TQFT and give a proof of Theorem 4.2.7. Next, we re-examine the Khovanov homology from this new more topological perspective, by re-examining the $n$-dimensional cube of cobordisms. What follows is a new quotient category of cobordisms, and finally another proof of invariance of the Khovanov bracket under R1.

\section{1 $\quad V$ is a Frobenius object}

Let $1_{R}$ denote the unit in the ring $R_{0}$, and $0_{R}$ denote the zero element in $R_{0}$. We will omit the subscripts $R$ from time to time where convenient. Recall $R_{0}$ is the graded $R$-module that is only non-trivial in $q$-grading 0 , where it is $R$. We let $\tau: W \otimes W^{\prime} \rightarrow W^{\prime} \otimes W$ which sends $w \otimes w^{\prime}$ to $w^{\prime} \otimes w$ be the twist map in $\operatorname{gr}(R$-mod $)$. Finally, recall we already have shown that $\left(\operatorname{gr}(R\right.$-mod $\left.), \otimes, R_{0}, \tau\right)$ is a symmetric monoidal category. 
We begin by defining the $\mathbb{Z}$-graded module $V$ from Chapter 4 . Recall $V$ is a $\mathbb{Z}$ graded module generated by $v_{+}$in degree +1 , and by $v_{-}$in degree -1 . It has a multiplication map and a co-multiplication map as follows.

$$
\begin{aligned}
& m: v_{+} \otimes v_{+} \mapsto v_{+} \quad \Delta: v_{+} \mapsto v_{+} \otimes v_{-}+v_{-} \otimes v_{+} \\
& v_{+} \otimes v_{-} \mapsto v_{-} \quad v_{-} \mapsto v_{-} \otimes v_{-} \\
& v_{-} \otimes v_{+} \mapsto v_{-} \\
& v_{-} \otimes v_{-} \mapsto 0
\end{aligned}
$$

Let the graded linear map $\tau: V \otimes V \rightarrow V \otimes V$ be the twist map, defined as $\tau(a \otimes b)=b \otimes a$. Recall (or see above) that the map $m$ is commutative, that is $m \tau=m$, and the map $\Delta$ is co-commutative, $\tau \Delta=\Delta$.

Thus, this being the case, we have a multiplication map $m$, a co-multiplication map $\Delta$, and a twist map $\tau$. These are commutative, and co-commutative respectively. Our next task is to define a unit and co-unit, which we want to satisfy the unit and co-unit relations. Let $\iota: R_{0} \rightarrow V$ be the unit and $\epsilon: V \rightarrow R_{0}$ be the co-unit, which are defined as follows. These maps, denoted $\iota$ and $\epsilon$ are defined on the generators of $V$ and $R_{0}$ as follows.

$$
\begin{aligned}
\iota: 1_{R} \mapsto v_{+} \quad \epsilon: & v_{+} \mapsto 0_{R} \\
& v_{-} \mapsto 1_{R}
\end{aligned}
$$

Proposition 7.1.1. $V$ with multiplication $m$, co-multiplication $\Delta$, unit $\iota$, co-unit $\epsilon$ and a twist map $\tau$ is a Frobenius object in $\operatorname{gr}(R$-mod $)$.

Proof. We need to check the following.

\section{The unit relation}

$$
m(\iota \otimes \mathrm{id})=\mathrm{id}=m(\mathrm{id} \otimes \iota)
$$

The co-unit relation

$$
(\epsilon \otimes \mathrm{id}) \Delta=\mathrm{id}=(\mathrm{id} \otimes \epsilon) \Delta
$$




\section{The Frobenius relation}

$$
(m \otimes \mathrm{id})(\mathrm{id} \otimes \Delta)=\Delta m=(\mathrm{id} \otimes m)(\Delta \otimes \mathrm{id})
$$

We will use a few properties of the tensor product, like $V \otimes R \cong V$, so one can write $1 \otimes v$ instead of $v$. For the first two relations, we only show one of the above equalities, as the other follows from commutativity $(m(\iota \otimes \mathrm{id})=m \tau(\iota \otimes \mathrm{id})=m(\mathrm{id} \otimes \iota))$, and co-commutativity $((\epsilon \otimes \mathrm{id}) \Delta=(\epsilon \otimes \mathrm{id}) \tau \Delta=(\mathrm{id} \otimes \epsilon) \Delta)$.

We begin with the unit relation. The following assignments show that $m(\iota \otimes$ id $)$ is the identity on each of the generators of $V$.

$$
\begin{aligned}
& v_{+}=1 \otimes v_{+} \stackrel{\iota \otimes \mathrm{id}}{\longmapsto} v_{+} \otimes v_{+} \stackrel{m}{\longmapsto} v_{+} \\
& v_{-}=1 \otimes v_{-} \stackrel{\iota \otimes \mathrm{id}}{\longmapsto} v_{+} \otimes v_{-} \stackrel{m}{\longmapsto} v_{-}
\end{aligned}
$$

Next, the co-unit relation. Again, we show that $(\epsilon \otimes$ id $) \Delta$ is the identity on each of the generators of $V$.

$$
\begin{gathered}
v_{+} \stackrel{\Delta}{\longmapsto} v_{+} \otimes v_{-}+v_{-} \otimes v_{+} \stackrel{\epsilon \otimes \mathrm{id}}{\longrightarrow} 0+1 \otimes v_{+}=v_{+} \\
v_{-} \stackrel{\Delta}{\longmapsto} v_{-} \otimes v_{-} \stackrel{\epsilon \otimes \mathrm{id}}{\longmapsto} 1 \otimes v_{-}=v_{-}
\end{gathered}
$$

Finally, we show the Frobenius relation. We must show this relation holds for three of the four generators, $v_{+} \otimes v_{+}, v_{+} \otimes v_{-}$and $v_{-} \otimes v_{-}$. That it holds on $v_{-} \otimes v_{+}$ follows from $v_{+} \otimes v_{-}$.

On $v_{+} \otimes v_{+}$.

$$
\begin{gathered}
v_{+} \otimes v_{+} \stackrel{\mathrm{id} \otimes \Delta}{\longmapsto} v_{+} \otimes v_{+} \otimes v_{-}+v_{+} \otimes v_{-} \otimes v_{+} \stackrel{m \otimes \mathrm{id}}{\longmapsto} v_{+} \otimes v_{-}+v_{-} \otimes v_{+} \\
v_{+} \otimes v_{+} \stackrel{m}{\longmapsto} v_{+} \stackrel{\Delta}{\longmapsto} v_{+} \otimes v_{-}+v_{-} \otimes v_{+}
\end{gathered}
$$




$$
v_{+} \otimes v_{+} \stackrel{\Delta \otimes \mathrm{id}}{\longmapsto} v_{+} \otimes v_{-} \otimes v_{+}+v_{-} \otimes v_{+} \otimes v_{+} \stackrel{\mathrm{id} \otimes m}{\longmapsto} v_{+} \otimes v_{-}+v_{-} \otimes v_{+}
$$

On $v_{+} \otimes v_{-}$.

$$
\begin{gathered}
v_{+} \otimes v_{-} \stackrel{\mathrm{id} \otimes \Delta}{\longmapsto} v_{+} \otimes v_{-} \otimes v_{-} \stackrel{m \otimes \mathrm{id}}{\longmapsto} v_{-} \otimes v_{-} \\
v_{+} \otimes v_{-} \stackrel{m}{\longmapsto} v_{-} \stackrel{\Delta}{\longmapsto} v_{-} \otimes v_{-} \\
v_{+} \otimes v_{-} \stackrel{\Delta \otimes \mathrm{id}}{\longrightarrow} v_{+} \otimes v_{-} \otimes v_{-}+v_{-} \otimes v_{+} \otimes v_{-} \stackrel{\text { id } \otimes m}{\longrightarrow} 0+v_{-} \otimes v_{-}
\end{gathered}
$$

On $v_{-} \otimes v_{-}$.

$$
\begin{aligned}
& v_{-} \otimes v_{-} \stackrel{\text { id } \otimes \Delta}{\longmapsto} v_{-} \otimes 0=0 \stackrel{m \otimes \mathrm{id}}{\longmapsto} 0 \\
& v_{-} \otimes v_{-} \stackrel{m}{\longmapsto} 0 \stackrel{\Delta}{\longmapsto} 0 \\
& v_{-} \otimes v_{-} \stackrel{\Delta \otimes \mathrm{id}}{\longmapsto} v_{-} \otimes 0=0 \stackrel{\text { id } \otimes m}{\longmapsto} 0
\end{aligned}
$$

Hence all three relations hold. Therefore, we have shown that $V$ is a Frobenius object in $\operatorname{gr}(R$-mod $)$.

For the rest of this chapter, we will work with the associated TQFT of $V$. That is, we will consider a functor, denoted $\mathcal{F}: 2 \mathbf{C o b} \rightarrow \operatorname{gr}(R$-mod $)$.

$$
(V, m, \Delta, \iota, \epsilon, \tau) \stackrel{(1+1) \text {-TQFT }}{\longmapsto} \quad[\mathcal{F}: 2 \mathbf{C o b} \rightarrow \operatorname{gr}(R \text {-mod })]
$$

\subsection{A topological n-dimensional cube of smooth- ings}

We have changed the notation from that in Chapter 6. Often from now on, when we are representing a particular cobordism, we will draw it from top to bottom, that is, the domain of the cobordism is at the top and the co-domain of the cobordism is at 
the bottom.

Let $D$ be a knot diagram. Recall the $n$-dimensional cube of smoothings is the cube where the vertices are total smoothings of $D$. We now think of each total smoothing as a 1-manifold! Rather than having graded linear maps on the edges though, we let the edges "be" cobordisms. That is, we replace the linear map $m: V \otimes V \rightarrow V$

with the pair of pants cobordism, $\{$, and the linear map $\Delta: V \rightarrow V \otimes V$ with the upside-down pair of pants, $\{\delta$, and identity maps with cylinders, $\square$. Thus, each edge is the disjoint union of cylinders and a pair of pants.

Therefore, we now think of the $n$-dimensional cube of smoothings as a cube of cobordisms defined directly from the knot diagram. Thus, we have found a topological way to view the $n$-dimensional cube of smoothings. We will look more at this topological perspective in Theorem 7.4.1.

\subsection{The Khovanov bracket is a chain complex}

We now have the grounding to prove that the Khovanov bracket, $\llbracket \cdot \rrbracket$, is a chain complex, and we have a TQFT, $\mathcal{F}$, instead of $V$. Now this becomes, in some sense, a topological problem. Recall from Chapter 4, what remains to show of Theorem 4.2.7 is that (without the minuses), every face of the cube commutes. We prove this now.

Before we begin the proof, it will be useful to think of the cube of cobordisms constructed in a different way. The final cube will be identical, but the different construction will simplify the proof.

Consider a knot diagram $D$ with $n$ crossings. Let $X_{i}$ denote the elementary 2-tangle containing the $i$ th crossing (see Subsection 2.1 in Chapter 2). That is, this is the intersection of the knot diagram $D$ with a 2-disc such that the boundary of the 2disc intersects the knot diagram $D$ exactly four times and the intersection contains exactly one crossing (the $i$ th). We also require that the $X_{i}$ are such that $X_{i} \cap X_{j}=\emptyset$ 
for $i \neq j$. We now define $\hat{D}$ to be the knot diagram without every $X_{i}$. That is,

$$
\hat{D}:=D \backslash\left(\sum_{i} X_{i}\right)
$$

which will be a collection of arcs in the plane as in the following example.
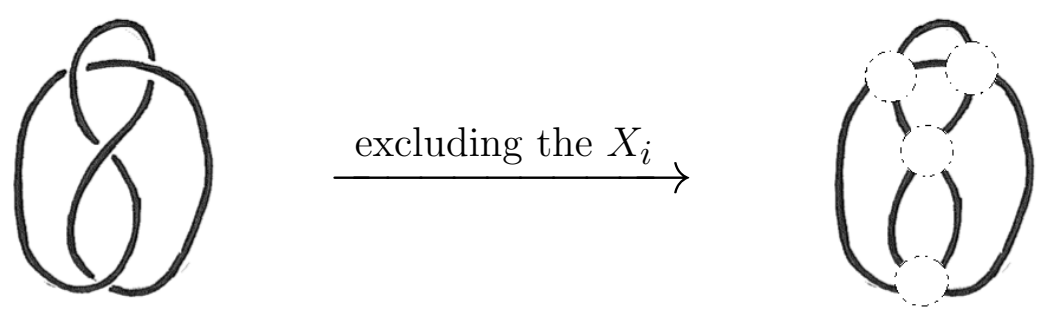

We now take the product of $\hat{D}$ with the unit interval $I, \hat{D} \times I$. Let $\alpha, \alpha^{\prime} \in\{0,1\}^{n}$ which differ at only the $i$ th place and consider the edge of the $n$-dimensional cube $S_{\alpha} \rightarrow S_{\alpha^{\prime}}$, represented by $\zeta$. The associated cobordism will be "identity arcs" on every crossing except the $i$ th crossing. This crossing will be replaced with a saddle, as follows. Hence, when we say we alter the $i$ th crossing, we mean that the $i$ th crossing that has a saddle in an associated cobordism (a cobordism from $\alpha \rightarrow \alpha^{\prime}$ where $\alpha$ and $\alpha^{\prime}$ differ at the $i$ th crossing).

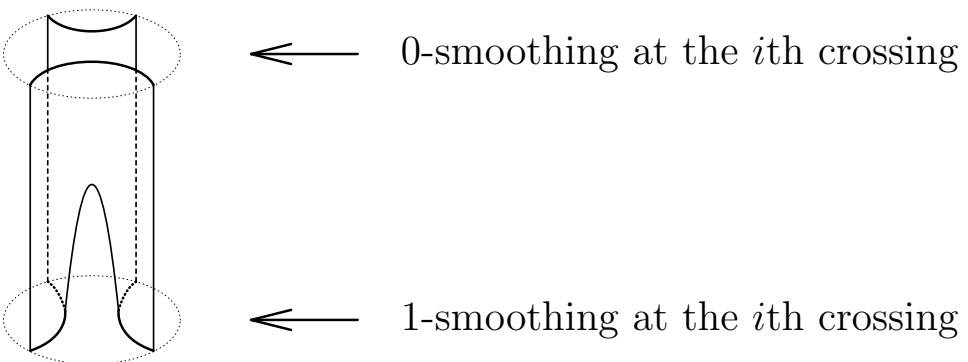

This saddle is an intersection of a pair of pants cobordism with a cylinder around this crossing. An advantage to drawing it like this is that we don't have to specify "which direction" the pair of pants is, and restrict our attention to these saddles. That 
is, in this case whether it is upside down or not. In terms of graded vector spaces, we don't have to specify whether the edge is a map $m: V \otimes V \rightarrow V$ or $\Delta: V \rightarrow V \otimes V$.

Proof of Theorem 4.2.7. All we need to do is see the equivalence in the following diagram, but first we will give some context. Let $D$ be a knot diagram with $n \geq 2$ crossings. Consider $\alpha \in\{0,1\}^{n}$ where the height of alpha is less than $n-1$, that is $|\alpha| \leq n-2$. Hence $\alpha$ will contain at least two 0 s - that is the smoothing $S_{\alpha}$ will contain two 0 -smoothings, which we label the $i$ th and $j$ th crossings where $i<j$. Consider a face of the $n$-dimensional cube that begins with this total smoothing, $S_{\alpha}$, where one path (a composition of two cobordisms) around the edge alters first the $i$ th crossing and then the $j$ th crossing, and the other path alters first the $j$ th crossing and then the $i$ th crossing. In order to visualise this, we take the intersection of the two cylinders containing the $i$ th crossing and the $j$ th crossing with the cobordisms on each path around the face.

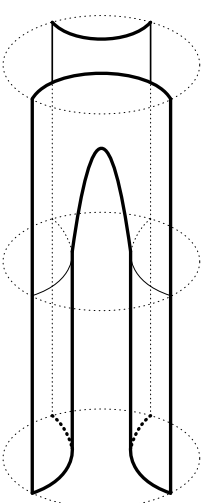

$i$ th crossing

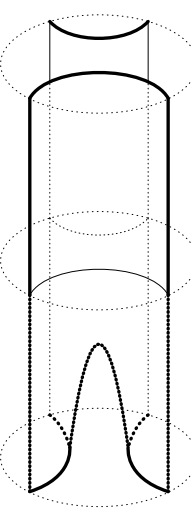

$j$ th crossing

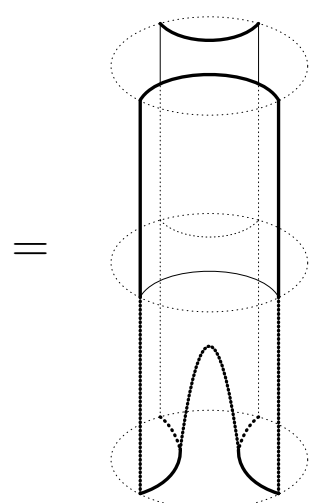

$i$ th crossing

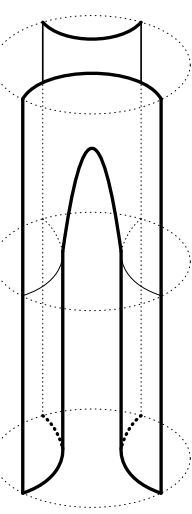

$j$ th crossing

Each side of this equality represents a different path around a face of the $n$-dimensional cube. Topologically, it is straightforward to see that the cobordisms on each side of the equality are homeomorphic, and since everything outside these two cylinders is identical, each side of the equation represents the same cobordism. Thus, each face 
of the $n$-dimensional cube commutes! As required.

\subsection{Some properties of the TQFT $\mathcal{F}$ associated to $V$}

We now will show some equivalences of the cobordisms under the functor $\mathcal{F}$. In other words, we are finding some cobordisms in the "kernel" of $\mathcal{F}$, that is, cobordisms that are sent to the 0 map in $\mathbf{g r}(R$-mod $)$. We will apply these results when proving the invariance of the Khovanov homology under $\mathbf{R} 1$ in the next section. This section uses many results from [2].

Theorem 7.4.1. The following equivalences hold after applying the TQFT to V.

S

$$
\mathcal{F}(\ldots))=0
$$

$\mathbf{T}$

$$
\mathcal{F}(\infty)=2
$$

4-Tu

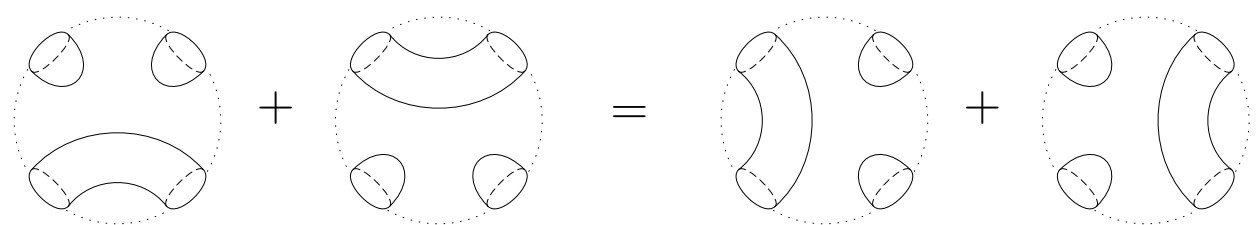


The $\mathbf{4}-\mathbf{T u}$ relation is written without the functor " $\mathcal{F}$ " which acts on each of the "cobordism-intersections" above for clarity.

The relation $\mathbf{S}$ implies that if a cobordism $M$ in $2 \mathbf{C o b}$ contains a sphere, then the functor $\mathcal{F}$ will assign $M$ the zero map in $\mathbf{g r}(R$-mod). The relation $\mathbf{T}$ implies that the functor $\mathcal{F}$ will assign to the disjoint union of a cobordism $M$ with a torus $T$ a map that is twice $\mathcal{F}(M)$. That is, $\mathcal{F}(M \amalg T)=2 \mathcal{F}(M)$, which sends $x \mapsto 2 \mathcal{F}(M)(x)$.

The relation 4-Tu is the most difficult to see. Consider the intersection of a cobordism $M$ and a sphere. If the boundary of the sphere and the cobordism intersect at exactly four places and the inside of the sphere looks like one of the summands in the picture above, without loss of generality, let it be the first. Let $M_{2}, M_{3}, M_{4}$ denote $M$ with the interior of the aforesaid sphere replaced with the second, third and fourth summands of the above equation. Then $\mathcal{F}(M)$ is equal to the map $\mathcal{F}\left(M_{3}\right)+\mathcal{F}\left(M_{4}\right)-\mathcal{F}\left(M_{2}\right)$.

Proof of Theorem 7.4.1. Let $\mathcal{F}$ be the TQFT assigned to $V$.

S All we must show is $\mathcal{F}(\subset \square)=\epsilon \iota=0$.

$$
\epsilon \iota(1)=\epsilon\left(v_{+}\right)=0
$$

$\mathbf{T}$ Recall the torus is the composition of cobordisms, $व$ \& $\{0$.

$$
\epsilon m \Delta \iota(1)=\epsilon m \Delta\left(v_{+}\right)=\epsilon m\left(v_{+} \otimes v_{-}+v_{-} \otimes v_{+}\right)=\epsilon\left(v_{-}+v_{-}\right)=1+1=2
$$

4-Tu This is more complicated than the others.

$$
\mathcal{F}(\Theta \Theta \Theta)+\mathcal{F}(\Theta \Theta)=\iota \otimes \iota \otimes\left(\Delta_{34} \iota\right)+\left(\Delta_{12} \iota\right) \otimes \iota \otimes \iota
$$

Hence, we get the following. We will write, for example, $v_{+} \otimes v_{-} \otimes v_{+} \otimes v_{+}$as $v_{+-++}$. The subscript on $\Delta$ refers to the placing of the component of the image 
of $\Delta$ in the tensor product. That is, $x \otimes \Delta_{13}\left(v_{+}\right) \otimes x^{\prime}=v_{+} \otimes x \otimes v_{-} \otimes x^{\prime}+v_{-} \otimes$ $x \otimes v_{+} \otimes x^{\prime}$.

$$
\begin{aligned}
\left(\iota \otimes \iota \otimes\left(\Delta_{34} \iota\right)+\left(\Delta_{12} \iota\right)\right. & \otimes \iota \otimes \iota)(1) \\
& =v_{+} \otimes v_{+} \otimes \Delta\left(v_{+}\right)+\Delta\left(v_{+}\right) \otimes v_{+} \otimes v_{+} \\
& =v_{++} \otimes\left(v_{+-}+v_{-+}\right)+\left(v_{+-}+v_{-+}\right) \otimes v_{++} \\
& =v_{+++-}+v_{++-+}+v_{+-++}+v_{-+++}
\end{aligned}
$$

Now for the other side of the equality.

$$
\mathcal{F}(\Theta \Theta)+\mathcal{F}(\Theta \Theta \Theta)=\iota \otimes\left(\Delta_{13} \iota\right) \otimes \iota+\iota \otimes \iota \otimes\left(\Delta_{24} \iota\right)
$$

Hence, we get the following.

$$
\begin{aligned}
\left(\iota \otimes\left(\Delta_{13} \iota\right) \otimes \iota+\iota \otimes \iota \otimes\right. & \left.\left(\Delta_{24} \iota\right)\right)(1) \\
& =v_{+} \otimes\left(\Delta_{13}\left(v_{+}\right)\right) \otimes v_{+}+v_{+} \otimes v_{+} \otimes\left(\Delta_{24}\left(v_{+}\right)\right) \\
& =\left(v_{++-}+v_{-++}\right) \otimes v_{+}+v_{+} \otimes\left(v_{++-}+v_{-++}\right) \\
& =v_{++-+}+v_{-+++}+v_{+++-}+v_{+-++}
\end{aligned}
$$

Hence, we have found that the maps are equal in $V$, as required.

We have only shown this for the case where all boundaries of 4-Tu lie on the codomain of the cobordism, but the relation still holds for all other arrangements of the boundary with respect to the in-boundaries and out-boundaries also satisfy the 4 - $\mathbf{T u}$ relation.

We note that this is not the way it is done in [2], but rather a somewhat backward way of what Dror Bar-Natan has done. Instead, he constructs a chain complex of 
1-manifolds with cobordisms as differentials from the topological $n$-dimensional cube we described above. He then finds a quotient of this category under the relations $\mathbf{S}, \mathbf{T}$ and 4-Tu and homotopy equivalence of chain complexes. The outcome of this construction is that each chain complex in this category is a link invariant. Hence, the true achievement is a geometric complex which is a link invariant, without ever establishing any algebraic structure. Only then does he apply the TQFT which we have denoted $\mathcal{F}$, to show how the theories agree. Therefore, in his paper, the above proposition shows that the functor $\mathcal{F}$ is well defined.

\subsection{First Reidemeister move (again)}

We now show invariance of the Khovanov homology under R1 using cobordisms. That is, we show a chain homotopy equivalence of the cochain complexes $\llbracket$ \ and $\llbracket \Omega \rrbracket[-1]$ (we have accounted for shifts here, for details refer to original proofs in Chapter 5). We apply the TQFT (implicitly) to each smoothing in each commuting square of the chain map, which is the identity outside all crossings and subsequently show that all maps (with respect to each smoothing $\alpha$ ) are homeomorphic, and hence equivalent as graded linear maps.

Proof. Recall that as $\Omega$ has one more positive crossing than $\Omega$, one shows that they are equivalent where $\Omega$ is under the shift $\cdot\{-1\}$. Recall also that we can write $\llbracket \Omega \rrbracket$ as a direct sum $\llbracket D_{0} \rrbracket \oplus \llbracket D_{1} \rrbracket$. So an element of $\llbracket \Omega$ is written as an ordered pair, and maps to and from $\llbracket \Omega \rrbracket^{r}$ are written as matrices. For the details, refer to Chapter 5 .

Let $A^{*}, B^{*}$ be chain complexes. Recall from Chapter 3 that $A^{*}$ and $B^{*}$ are chain homotopy equivalent if there exist chain maps $f^{*}: A^{*} \rightarrow B^{*}$ and $g^{*}: B^{*} \rightarrow A^{*}$ such that $g^{*} f^{*}$ is homotopic to $\mathrm{id}_{A}$ and $f^{*} g^{*}$ is homotopic to $\mathrm{id}_{B}$. Also, recall that chain homotopy equivalence is an equivalence relation on chain complexes. Moreover, chain homotopy equivalent chain complexes have the same homology.

In the following diagrams, we omit the functor $\mathcal{F}$ on all cobordisms, which is standard 
notation.

We want to show that $\llbracket \Omega$ and $\llbracket \Omega \rrbracket\{-1\}$ are homotopy equivalent. That is, that there exists chain maps $F: \llbracket D^{\prime} \rrbracket^{*} \rightarrow \llbracket D \rrbracket^{*-1}$ and $G: \llbracket D \rrbracket \rightarrow \llbracket D^{\prime} \rrbracket$ such that $G F \sim \operatorname{id}_{D^{\prime}}$ and $F G \sim \operatorname{id}_{D}$. We first define our $F:=\left[\begin{array}{c}F_{0} \\ F_{1}\end{array}\right]$ and $G:=\left[\begin{array}{ll}G_{0} & G_{1}\end{array}\right]$.

Let $F_{1}: \llbracket D^{\prime} \rrbracket^{r} \rightarrow \llbracket D_{1} \rrbracket^{r-1}\{1\}$ be zero and let $F_{0}: \llbracket D^{\prime} \rrbracket^{r} \rightarrow \llbracket D_{0} \rrbracket^{r}$ be the following chain map/cobordism.

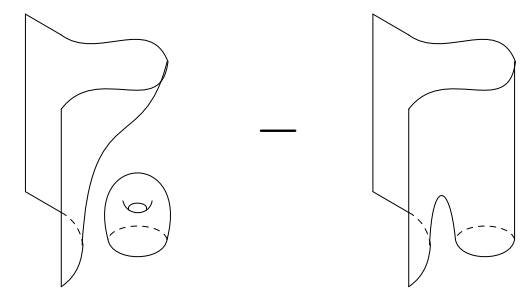

Let $G_{1}: \llbracket D_{1} \rrbracket^{r-1} \rightarrow \llbracket D^{\prime} \rrbracket^{r}$ be zero and let $G_{0}: \llbracket D_{0} \rrbracket^{r-1} \rightarrow \llbracket D^{\prime} \rrbracket^{r}$ be the following chain map/cobordism.

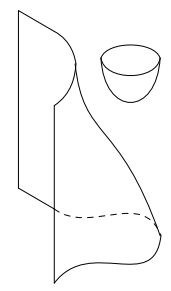

Showing $G F \sim \mathrm{id}$, and in fact we have an equality. $\mathbf{G F}=\mathrm{id} \Omega$ 

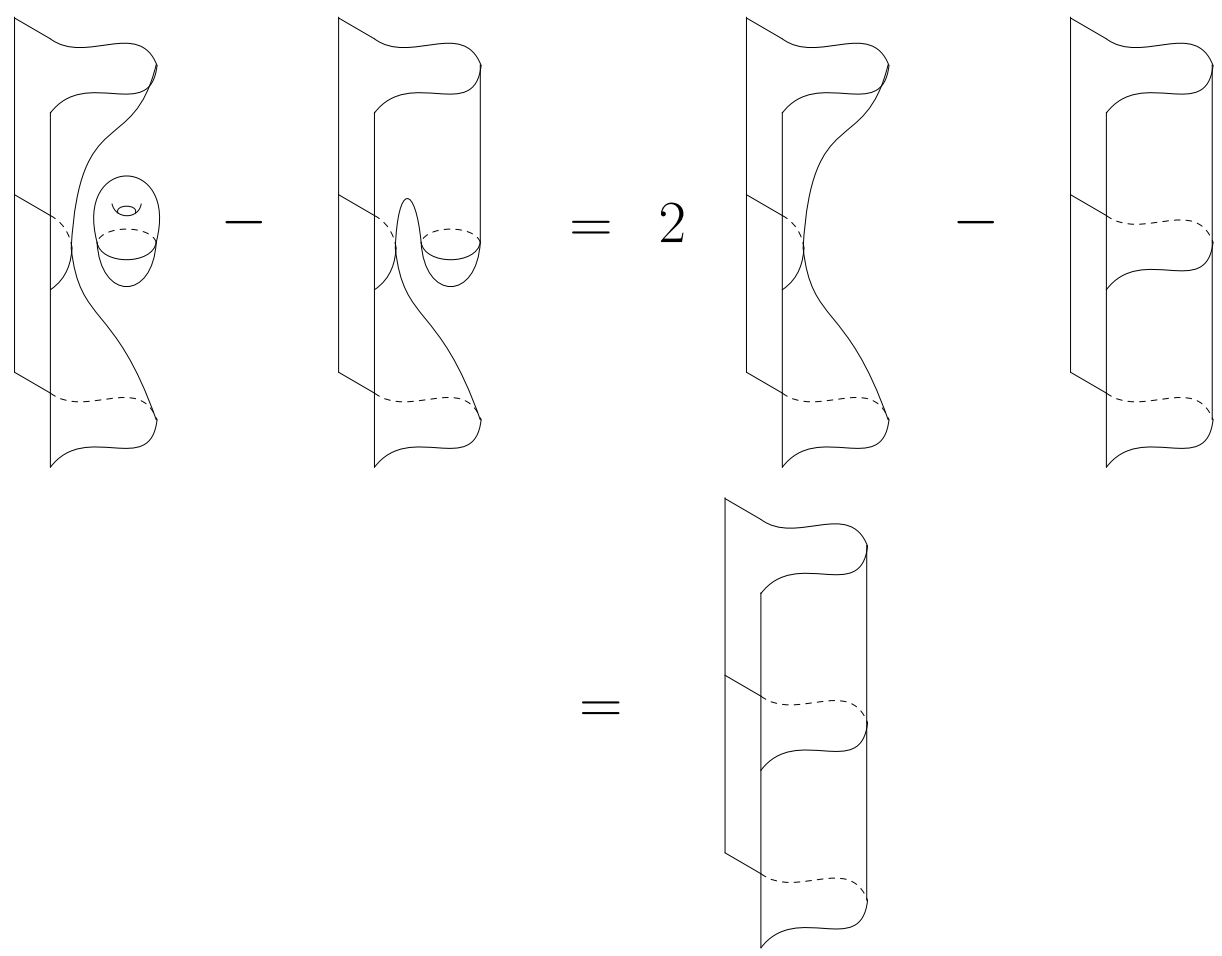

As for $F G \sim \operatorname{id}_{D}$, we need to define maps $H: \llbracket D \rrbracket \rightarrow \llbracket D \rrbracket$. Hence, this will be a linear map defined by the matrix $H:=\left[\begin{array}{ll}0 & h \\ 0 & 0\end{array}\right]$. We begin by reminding ourselves of the differentials first.

We know the differential $d=\left[\begin{array}{cc}d_{0} & 0 \\ \hat{m} & -d_{1}\end{array}\right]$, where $\hat{m}: \llbracket D_{0} \rrbracket^{r} \rightarrow \llbracket D_{1} \rrbracket^{r}$ is the following chain map/cobordism.

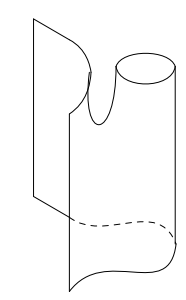

The map $-h: \llbracket D_{1} \rrbracket^{r} \rightarrow \llbracket D_{0} \rrbracket^{r}$ is the following chain map/cobordism. 


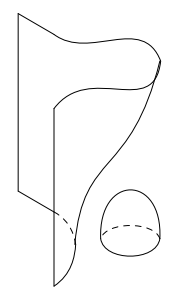

We now need to check that $F G-$ id $=H d+d H$. In other words, we need the following.

$$
\left[\begin{array}{cc}
F_{0} G_{0} & 0 \\
0 & 0
\end{array}\right]-\left[\begin{array}{cc}
\text { id } & 0 \\
0 & \text { id }
\end{array}\right]=\left[\begin{array}{cc}
-h m & h d_{1} \\
0 & 0
\end{array}\right]+\left[\begin{array}{cc}
0 & -d_{0} h \\
0 & -m h
\end{array}\right]
$$

We will show these one by one, starting with the easiest.

Firstly, that $-\mathrm{id}=-m h$ can be seen from the diagram below.

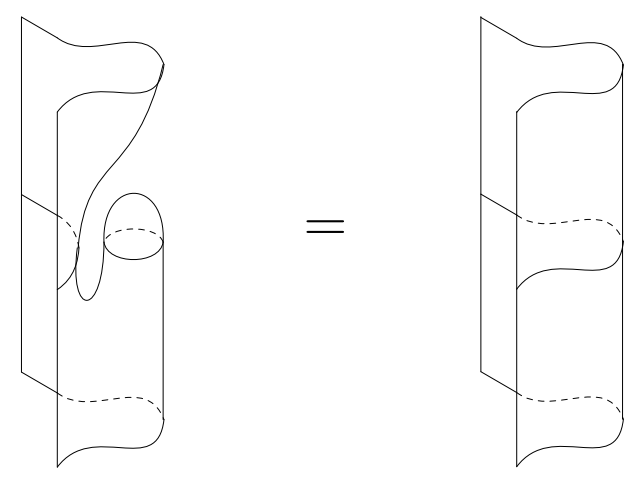

Next, we show $0=+h d_{1}-d_{0} h$. This follows from a similar construction used in Section 7.3 - that if two cobordisms are identical outside two cylinders and homeomorphic inside the cylinders, then they represent the same cylinder. We will denote the "first" cylinder, that is the vertical cylinder of the crossing in question, by $W$. Inside $W$, the differentials $d_{0}$ and $d_{1}$ will be identities. Hence, we have the following homeomorphism $\left.h d_{1}\right|_{W}=\left.d_{0} h\right|_{W}$. 


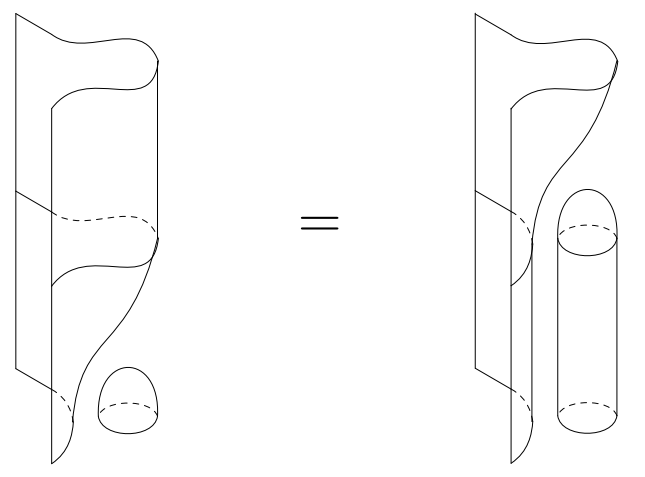

The differentials $d_{0}$ and $d_{1}$ are differentials in $\llbracket D_{0} \rrbracket$ and $\llbracket D_{1} \rrbracket$ respectively. Suppose $-h^{r}: \llbracket D_{1} \rrbracket^{r-1} \rightarrow \llbracket D_{0} \rrbracket^{r-1}$, and let $\backslash W$ mean the cobordism excluding its intersection with $W$. We must show $\left.h^{r+1} d_{1}^{r-1}\right|_{\backslash W}=\left.d_{0}^{r-1} h^{r}\right|_{\backslash W}$. Now we can see that $d_{1}$ and $d_{0}$ are of the same degree, it is straightforward to see that for each summand $\left(d_{0}\right)_{\zeta}$ of $d_{0}$ and $\left(d_{1}\right)_{\zeta}$ of $d_{1},\left(d_{0}\right)_{\zeta}=\left(d_{1}\right)_{\zeta}$ outside of $W$. This shows $\left.h^{r+1} d_{1}^{r-1}\right|_{\backslash W}=\left.d_{0}^{r-1} h^{r}\right|_{\backslash W}$, as required.

Finally, we must check that $F_{0} G_{0}-\mathrm{id}_{D_{0}}+h m=0$. This follows from the 4 -Tu relation. The first line is $F_{0} G_{0}+h m$.

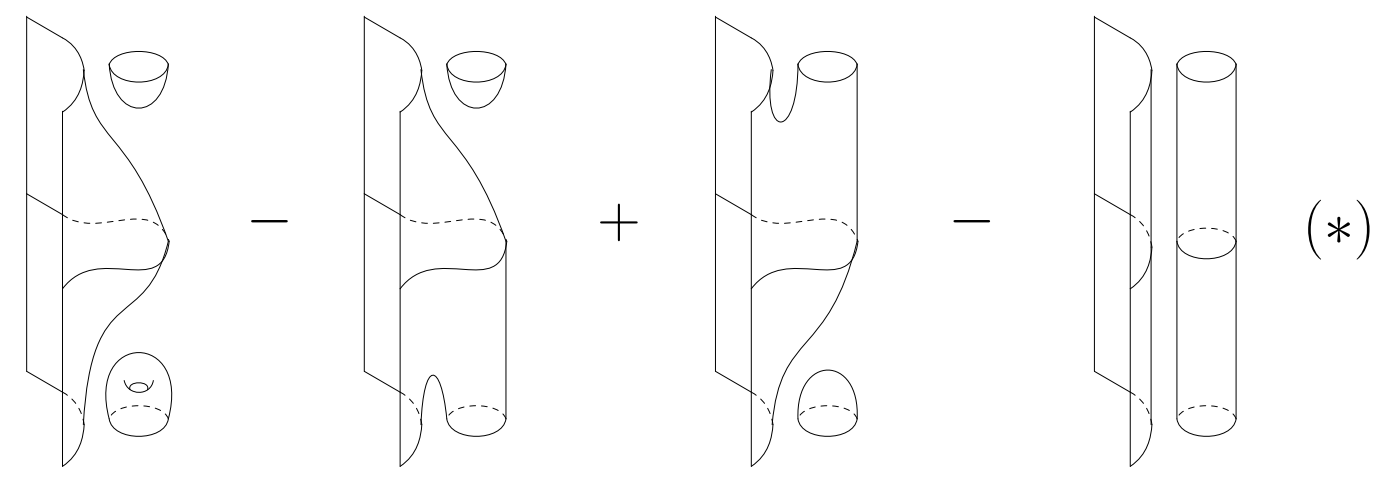

We apply the 4-Tu relation to the first cobordism. The following is the first cobordism without a ball. Inside the ball looks like a summand of the $\mathbf{4}$-Tu relation, which we will now apply. 

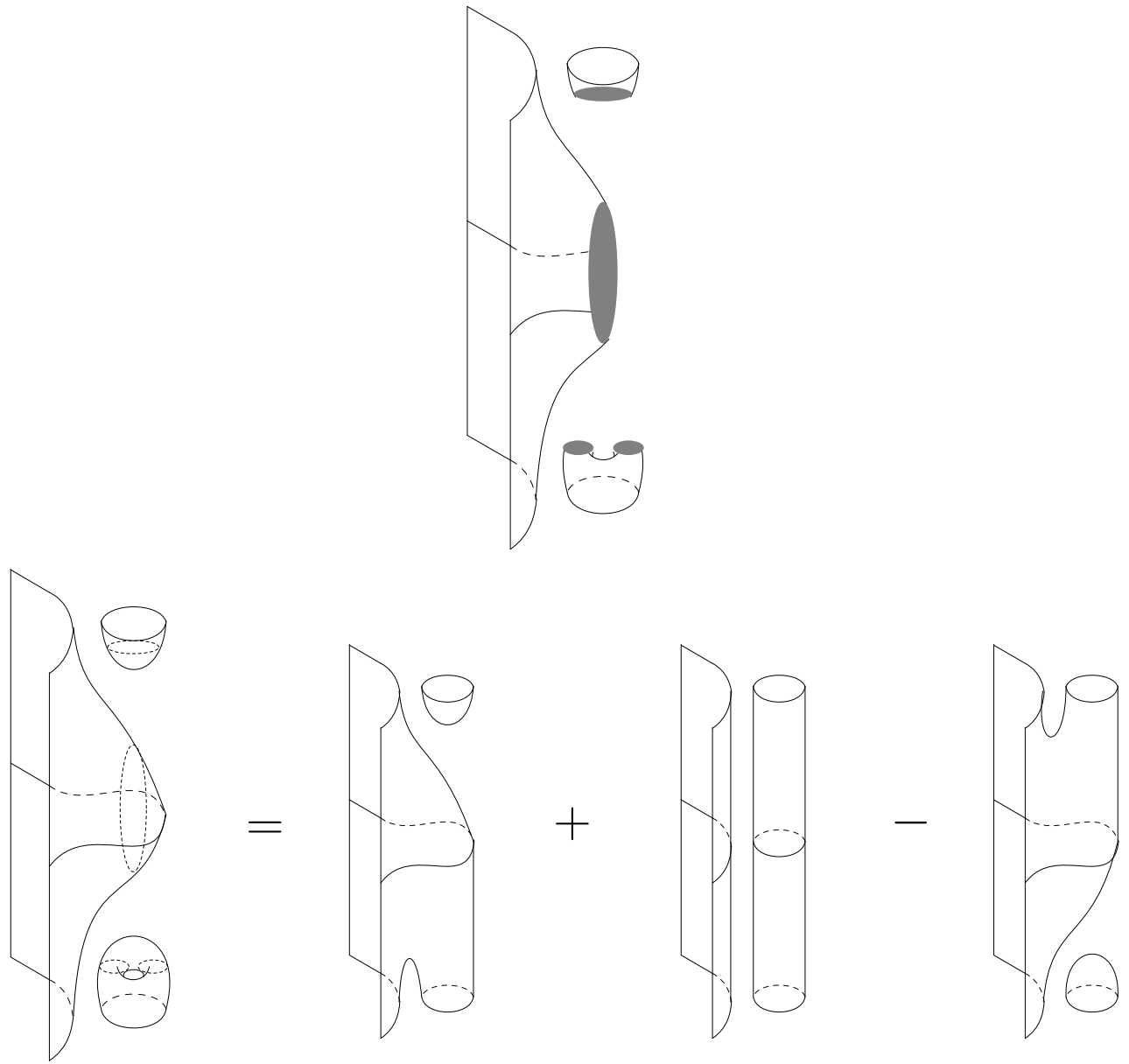

Hence, substituting the right-hand side of this equation into $(*)$, we find that everything cancels. Therefore, the equation $(*)$ is equal to 0 , which is what we required.

This shows invariance under $\mathbf{R} \mathbf{1}$. 


\section{Bibliography}

[1] Dror Bar-Natan, On Khovanov's categorification of the Jones polynomial, arXiv:math/0201043.

[2] Dror Bar-Natan, Khovanov's homology for tangles and cobordisms, arXiv:math/0410495.

[3] G. Burde and H. Zieschang, Knots, Walter de Gruyter \& Co., Berlin, 1985.

[4] Allen Hatcher, Algebraic Topology Cambridge University Press, New York, 2001.

[5] Morris W. Hirsch, Differential Topology, Springer-Verlag, New York, 1976

[6] Mikhail Khovanov, A categorification of the Jones polynomial, arXiv:math/9908171.

[7] Joachim Kock, Frobenius Algebras and 2D Topological Quantum Field Theories London Mathematical Society student texts; 59 Cambridge University Press, Cambridge, UK, 2004.

[8] P. B. Kronheimer and T. S. Mrowka, Khovanov homology is an unknot-detector, arXiv:math/1005.4346.

[9] Saunders Mac Lane, Garrett Birkhoff, Algebra, The Macmillan Company, New York, 1967.

[10] Saunders Mac Lane, Categories for the Working Mathematician, 2nd ed., Springer-Verlag, New York, 1998. 
[11] Serge Lang, Algebra, Addison Wesley Publishing Co., Reading, Massachusetts, 1965

[12] H. F. Trotter, Non-invertible knots exist, Topology, Vol. 2, pp. 275-280, Pergamon Press, 1964.

[13] Paul Turner, Five Lectures on Khovanov Homology, arXiv:math/0606464.

[14] Charles Weibel, Introduction to Homological Algebra Cambridge University Press, 1994 